
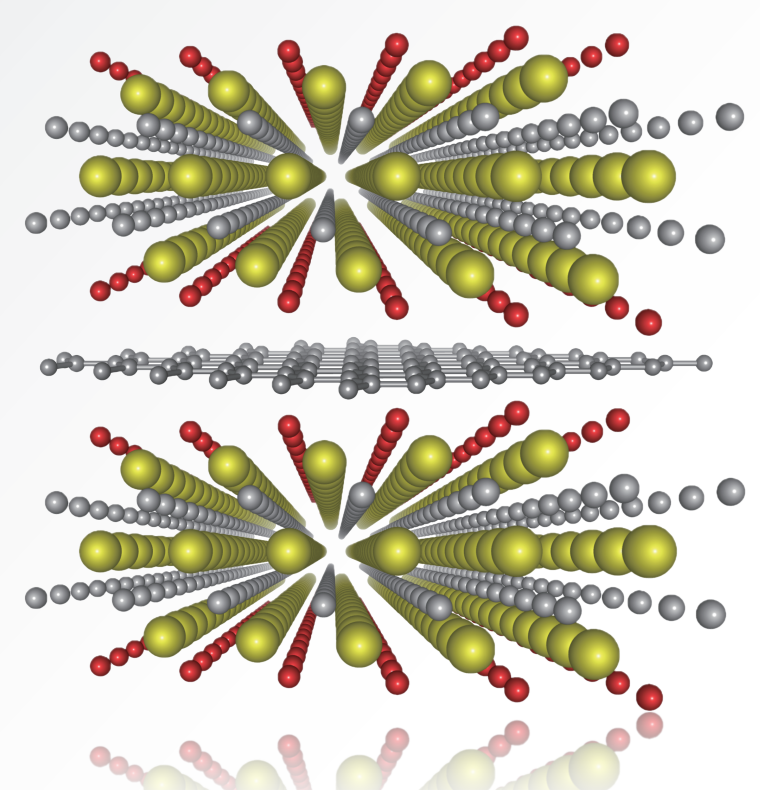


\section{PRINTABLE TWO-DIMENSIONAL MATERIALS FOR ENERGY STORAGE DEVICES}




\section{Graduation Committee}

\section{Chairman:}

Prof.dr. J.L. Herek

University of Twente

Promotor:

Prof.dr.ir. J.E. ten Elshof

University of Twente

\section{Committee Members:}

Prof. dr. ir. M. Wagemaker

Delft University of Technology

Prof. dr. ir. B. J. Kooi

University of Groningen

Prof. dr. ir. M. Huijben

University of Twente

Prof. dr. ir. R. G. H. Lammertink

University of Twente

Prof. dr. ir. R. Akkerman

University of Twente

The work described in this thesis was carried out in the Inorganic Materials Science (IMS) group, MESA+ Institute for Nanotechnology, Faculty of Science and Technology, University of Twente, The Netherlands. This research was financially supported by China Scholarship Council (CSC, 201608340058).

Title: Printable Two-Dimensional Materials for Energy Storage Devices

Ph.D. Thesis, University of Twente, Enschede, The Netherlands

Cover: Illustration of MXene/Graphene/MXene nanosheets heterostructure

Cover design: Yang Wang

Printed by: Gildeprint - Enschede

ISBN: 978-90-365-5050-5

DOI: $10.3990 / 1.9789036550505$

(C) 2020 Yang Wang, The Netherlands. All rights reserved. No parts of this thesis may be reproduced, stored in a retrieval system or transmitted in any form or by any means without permission of the author. Alle rechten voorbehouden. Niets uit deze uitgave mag worden vermenigvuldigd, in enige vorm of op enige wijze, zonder voorafgaande schriftelijke toestemming van de auteur. 


\title{
PRINTABLE TWO-DIMENSIONAL MATERIALS FOR ENERGY STORAGE DEVICES
}

\author{
DISSERTATION \\ to obtain \\ the degree of doctor at the Universiteit Twente, \\ on the authority of the rector magnificus, \\ Prof.dr. T.T.M. Palstra, \\ on account of the decision of the Doctorate Board \\ to be publicly defended \\ on Wednesday 7 October 2020 at 14.45 uur
}

by

\section{Yang Wang}

Born on the 18 December 1991

in Anhui, China 
This dissertation has been approved by the promotor:

Prof. dr. ir. J.E. ten Elshof 
We are trying to prove ourselves wrong as quickly as possible, because only in that way can we find progress.

Richard P. Feynman

Dedicated to my beloved wife and parents 



\section{Contents}

Chapter 1 Introduction ....................................................................................................... 1

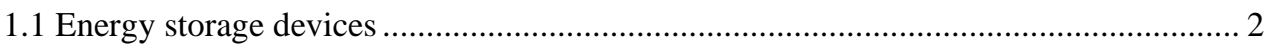

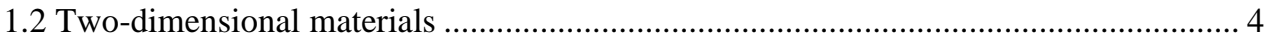

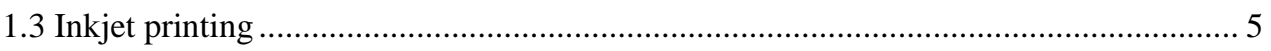

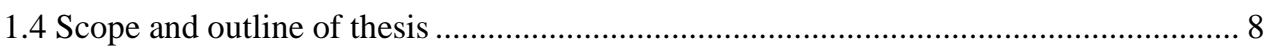

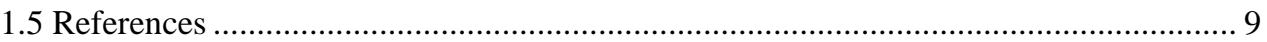

Chapter 2 Inkjet printing of $\delta-\mathrm{MnO}_{2}$ nanosheets for flexible solid-state microsupercapacitor .......................................................................................................................... 13

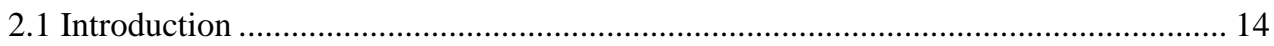

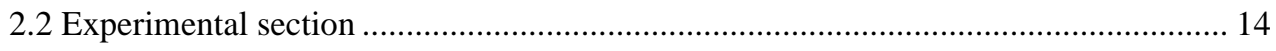

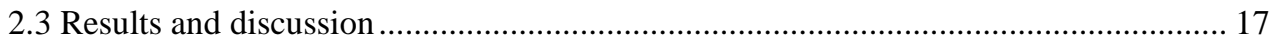

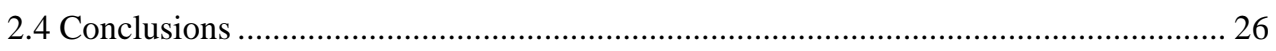

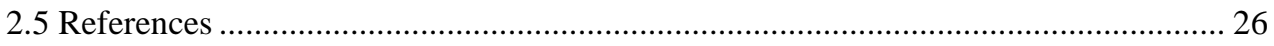

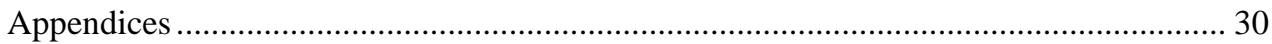

Chapter 3 Defect engineering of $\mathrm{MnO}_{2}$ nanosheets by substitutional doping for printable solid-state micro-supercapacitors ............................................................................ 35

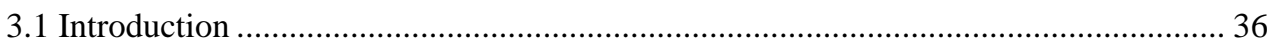

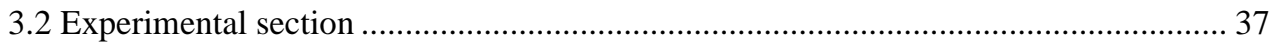

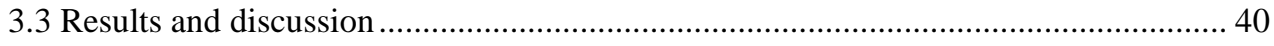

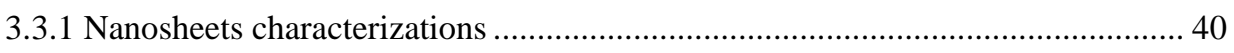

3.3.2 Formulation of water-based printable nanosheets inks ........................................ 43

3.3.3 Three-electrode measurement of printed electrodes .............................................. 44

3.3.4 First principle calculations ............................................................................... 45

3.3.5 Electrochemical performance of printed MSCs .................................................. 46

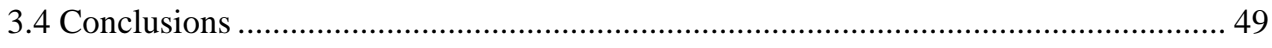

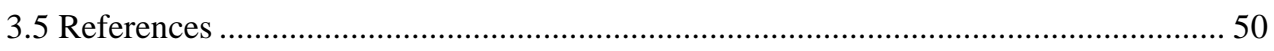

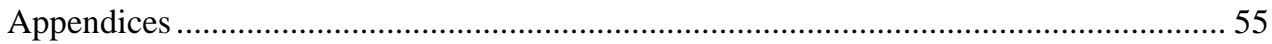


Chapter 4 Tunable capacitance in all-inkjet-printed nanosheet heterostructures......... 65

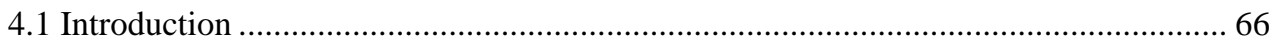

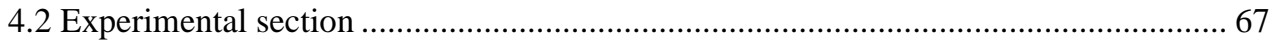

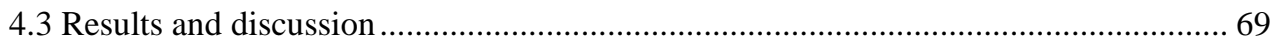

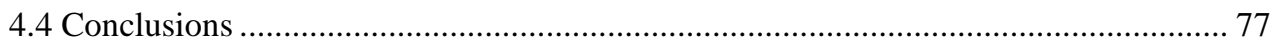

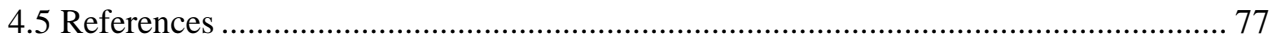

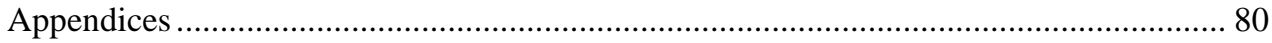

Chapter 5 Printed two-dimensional $\mathrm{V}_{2} \mathrm{O}_{5}$ /MXene heterostructure cathode for lithium-

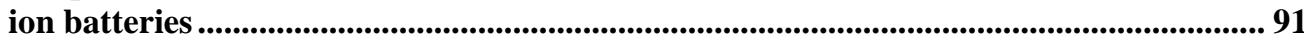

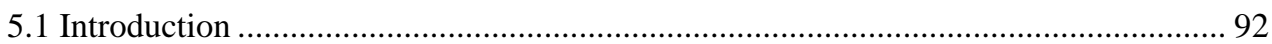

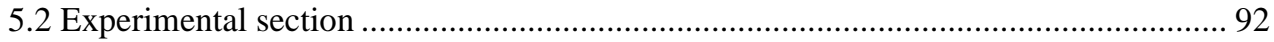

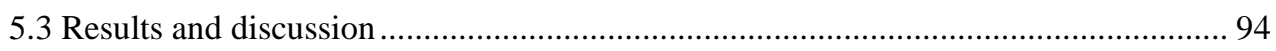

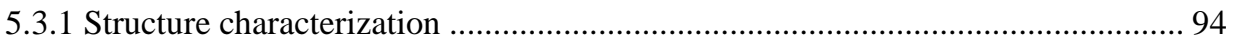

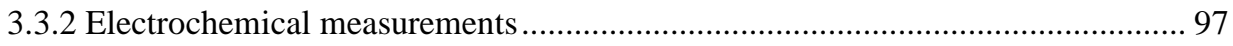

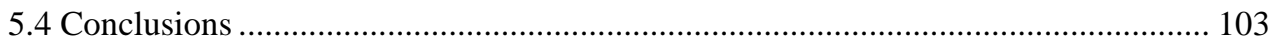

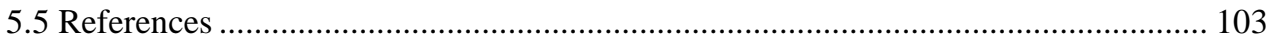

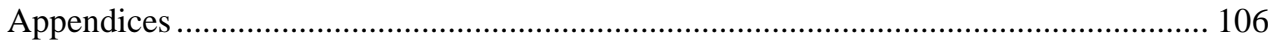

Chapter 6 Challenges and opportunities ........................................................... 111

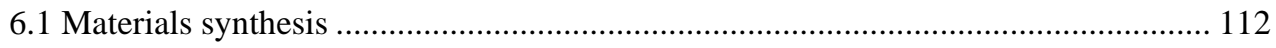

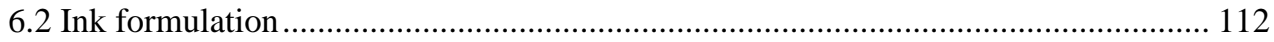

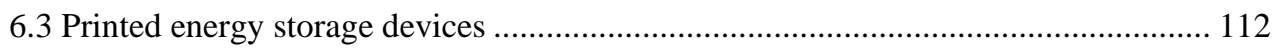

6.4 Printed energy storage devices performance .................................................... 114

Summary ..................................................................................................................................... 115

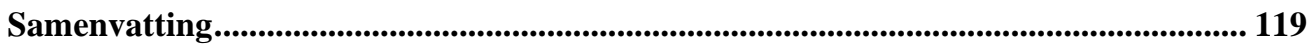

List of publications ...................................................................................................................... 123

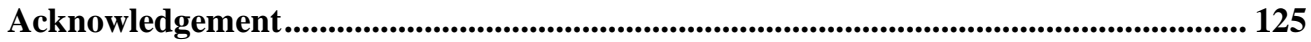




\section{Chapter 1}

\section{Introduction}

First, energy storage devices are discussed in this chapter. An introduction of twodimensional (2D) nanomaterials is presented. The mechanics and challenges of inkjet printing technology are discussed. Applications including electrochemical capacitors and lithium-ion batteries are introduced. The scope and outline of this thesis are described at the end of the chapter.

This chapter is based in part on the following publications:

1. ten Elshof, J. E.;* Wang, Y.,* Advances in ink-jet printing of $\mathrm{MnO}_{2}$-nanosheet based pseudocapacitors. Small Methods 2019, 3 (8), 1800318. (*corresponding author)

2. Timmerman, M. A.; Xia, R.; Le, P. T. P.; Wang, Y.; ten Elshof, J. E., Metal oxide nanosheets as 2D building blocks for the design of novel materials. Chemistry -A European Journal 2020, 26, 9085-9098. 


\subsection{Energy storage devices}

The rapid penetration of portable consumer electronics and autonomous devices in our society has led to a growing need for small-scale electrochemical energy storage (EES) devices to provide them with energy. Electrochemical capacitors which also called supercapacitors (SCs) and rechargeable batteries, are the two main energy storage devices. Supercapacitors can be divided into two main classes, i.e. electrochemical double layer (EDL) capacitors and pseudocapacitors (Figure 1.1). ${ }^{1,2}$ The energy density of EDL capacitors is limited to the charge that can be stored in the so-called electrochemical double layer that is present in the electrolyte near the electrode surfaces. EDL capacitors typically employ metallic or graphitic electrodes. Pseudocapacitors make use of fast and reversible faradaic reactions at the electrode surface. This requires the use of specific materials with a high concentration of surface redox sites. Since the EDL effect is also operative in pseudocapacitors, they can achieve significantly higher energy densities than EDL capacitors can.

While the energy density of supercapacitors is considerably smaller, supercapacitors are appreciated for their high power density, long cycle life and safe operation. Supercapacitors are particularly useful in applications where a large amount of electrical energy needs to be stored or delivered quickly. Supercapacitor technology is developing quickly because of their increasing need in the ongoing electrification of our society. Next to their use in electrical vehicles, especially buses that have to stop frequently where charging facilities can be provided, energy storage based exclusively on ultracapacitors becomes viable. It can also be foreseen that the need for small-sized supercapacitors in electrical appliances, autonomous devices, and flexible electronics will increase further. In order to enable the fabrication of such high-performance flexible micro-supercapacitor (MSC) devices on large scale at low cost, it is necessary to develop new scalable, versatile, solution-based methods and printing techniques.

The currently dominant energy sources are rechargeable lithium-ion (Li-ion) batteries, which hold high energy density. They are powering almost all forms of portable consumer electronics and electric vehicles. The first functional lithium battery was demonstrated by M. Stanley Whittingham in the early 1970s, who showed that lithium can be intercalated into the $\mathrm{Li}_{\mathrm{x}} \mathrm{TiS}_{2}$ materials over the whole stoichiometric range $(0<\mathrm{x} \leq 1)$ with a small lattice expansion effect. ${ }^{3}$ In 1979/1980, John B. Goodenough and co-workers at Oxford University discovered that $\mathrm{Li}_{x} \mathrm{CoO}_{2}$ materials with van der Waals gaps between $\mathrm{CoO}_{2}$ layers could be used as cathode materials without dramatic lattice expansion during Li-ions intercalation. ${ }^{4}$ Akira Yoshino found that certain qualities of petroleum coke were stable under the required electrochemical conditions in 1985. ${ }^{5,6}$ The discoveries from John B. Goodenough, M. Stanley Whittingham and Akira Yoshino had a tremendous impact on our human society. 


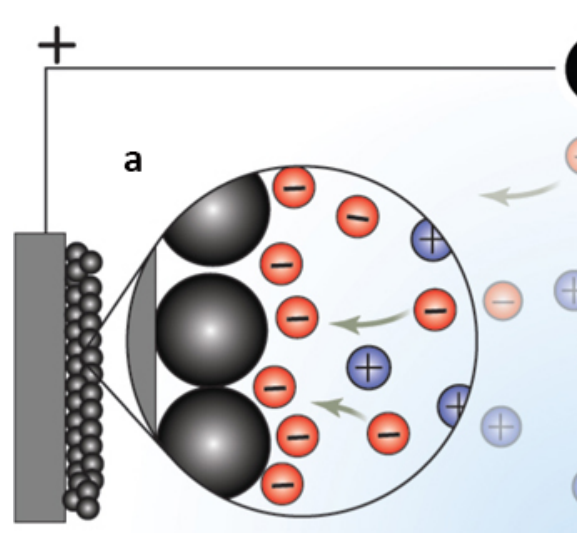

b

\section{v}

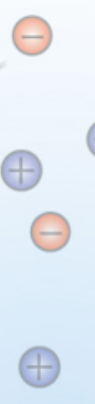

$\leftarrow$

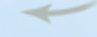

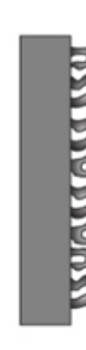
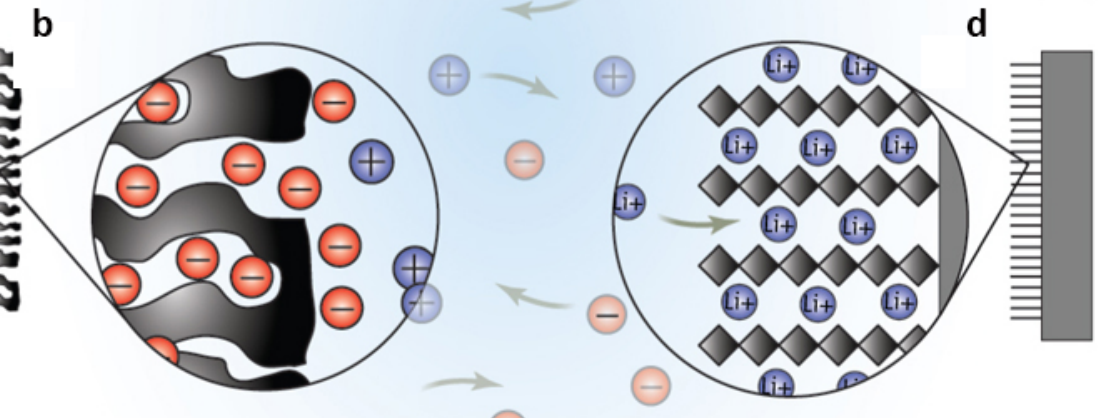

C

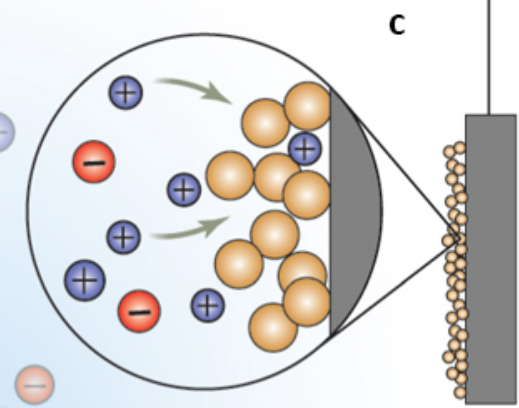

d

Figure 1.1 Illustration of different mechanisms of supercapacitors. Double-layer capacitance mechanisms include of (a) carbon particles or (b) porous carbon. Pseudocapacitive mechanisms include (c) redox and (d) intercalation pseudocapacitance. Adapted from ref. 2. Reprinted with permission from AAAS. Credit: KRISTY JOST/DREXEL.

The working principle of Li-ion battery refers to the processes of $\mathrm{Li}$-ion intercalation and deintercalation into electrode materials which is diffusion-controlled and slow process (Figure 1.2). The main components of a Li-ion battery are the anode and cathode, which are connected to an electric circuit, and separated by an electron-insulating separator with electrolyte that can accommodate charged species. To drive the electric circuit, a Li-ion deintercalation process takes place at the anode accompanying with electrons moving from the anode through the circuit. A complementary Li-ion intercalation process takes place at the cathode which is replenished by adding electrons from the external circuit. These processes are reversible to charge the Li-ion battery. The batteries voltage depends on the potential difference of electrodes and electrolyte. 


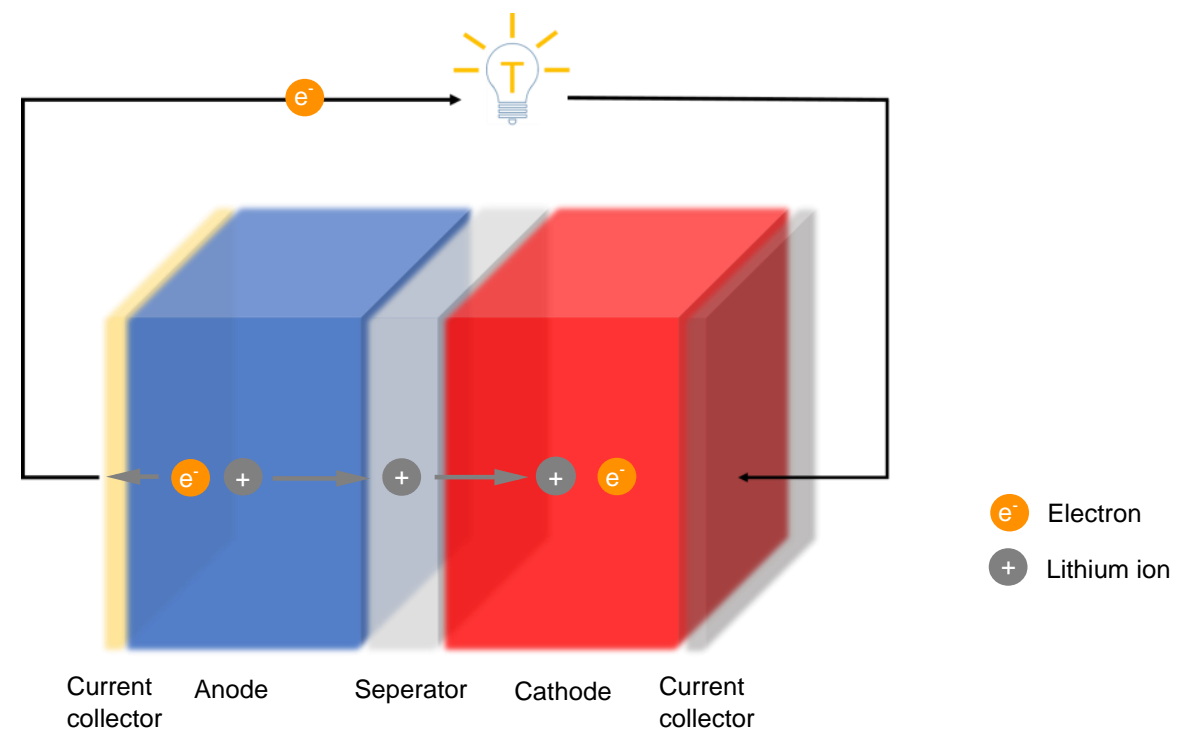

Figure 1.2 Schematic illustration of ion transfer in lithium-ion battery.

a

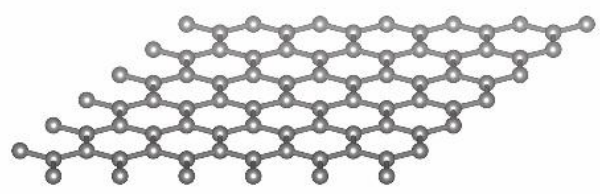

C

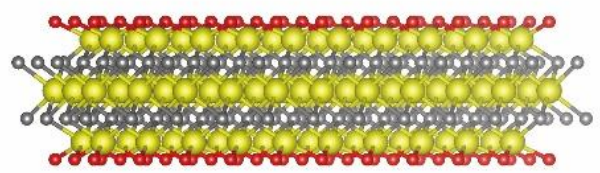

b

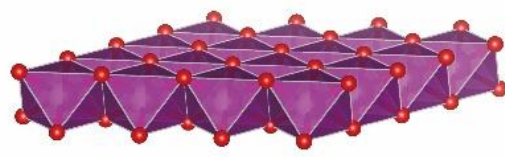

d

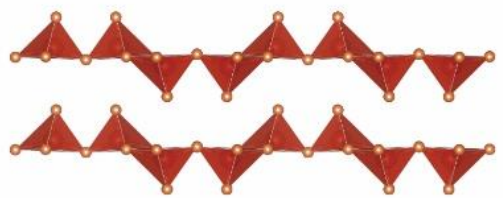

Figure 1.3 Crystal structure of (a) graphene, (b) $\mathrm{MnO}_{2}$ nanosheet, (c) $\mathrm{Ti}_{3} \mathrm{C}_{2} \mathrm{~T}_{\mathrm{x}}$ nanosheet and (d) $\mathrm{V}_{2} \mathrm{O}_{5}$ nanosheet.

\subsection{Two-dimensional materials}

Two-dimensional (2D) materials, or nanosheets, are a class of nanomaterials that draws more and more attention since graphene was discovered (Figure 1.3). ${ }^{7}$ These $2 \mathrm{D}$ materials exhibit a sheet-like structure, hence the name nanosheets, with lateral dimensions of tens to hundreds of nanometers, even up to micrometers, and thicknesses below than $5 \mathrm{~nm} .^{8}$ Owing to the 
effect of spatial confinement in one dimension, nanosheets can exhibit a variety of electronic, chemical and optical properties that are not present in their layered bulk counterparts. ${ }^{9}$

A couple of advantages arises from the two-dimensional nature of nanosheets. Firstly, the electrons are confined in a thin (nanoscale) region. ${ }^{9}$ Their electrons are thus confined to a two-dimensional lattice plane, which provides an ideal model system for fundamental studies in condensed matter physics, but also for development of small (opto-)electronic devices. Since nanosheets have strong in-plane bonds and the sheet is atomically thin, they tend to show a combination of high mechanical strength, flexibility and optical transparency, which are all highly desirable properties for utilization in various types of devices. ${ }^{10}$ Their atomic thicknesses results in very specific surface areas, ${ }^{11}$ which is a very important property for applications in which the surface area is relevant, such as batteries and supercapacitors. ${ }^{12}$ Furthermore, the aqueous solution-based dispersions of nanosheets are suitable precursors for the fabrication of nanosheet-based films using simple methods like spin-coating and ink jet printing, usable in applications such supercapacitors and batteries. And finally, the fact that all atoms are surface atoms provides a handle to regulate the properties and functionalities of nanosheets by means of surface modification and functionalization, for example with graphene oxide, substitutional element doping, or strain and phase engineering. ${ }^{13}$

\subsection{Inkjet printing}

Due to the simple, versatile and low-cost features, ink-jet printing (IJP) shows great potential for supercapacitors fabrication with desired configuration like interdigitated, asymmetric, etc. Since nanosheets are typically obtained in the form of homogeneous aqueous colloidal solutions, these colloids can serve as starting point for the formulation of water-based inks that are suitable for IJP small devices on arbitrary substrates. ${ }^{14-16}$ The printing process consists of jetting droplets from a nozzle under driving pressure, followed by impaction and deposition of droplets on a substrate. The morphology of printed patterns depends on the printing apparatus, ink formulation, substrate interface properties and post-treatment process. Thus far, inkjet printing has been used to fabricate electronic devices like field effect transistors, ${ }^{17,18}$ solar cells, ${ }^{19}$ organic light-emitting diodes ${ }^{20}$ and electrochemical energy storage devices. $^{21}$

Depending on the printing mechanism, an inkjet printer can be operated in two different modes: continuous inkjet (CIJ) and drop-on-demand (DOD) printing, as shown in Figure 1.4. The CIJ mode is a process in which a continuous stream of droplets is jetted by the printer head nozzles. The jetted droplets are then subjected to an electrostatic field, which directs them towards the substrate. All undesired droplets are directed to a recycling system. The DOD mode is a process in which droplets are jetted only when desired. They are deposited onto a substrate in a predesigned pattern. Because the recycling system may contaminate the 
ink, the CIJ mode is not used very often. DOD mode printers are the majority inkjet printers for printed electronics manufacturing. Thermal and piezoelectric actuation are the two main actuation mechanisms of a DOD inkjet printer. In the thermal process, a resistive element is activated that forms a gas bubble inside the reservoir, leading to ejection of a droplet via the nozzle. As this point, the resistive element is turned off and the vacuum draws new ink to refill the reservoir. In the piezoelectric process, a voltage pulse is applied to the piezoelectric reservoir walls, which creates a mechanical pressure. The pressure squeezes the functional ink through the nozzle onto the substrate. When the voltage pulse is switched off, the vacuum created by reservoir walls will draw new ink into the reservoir.
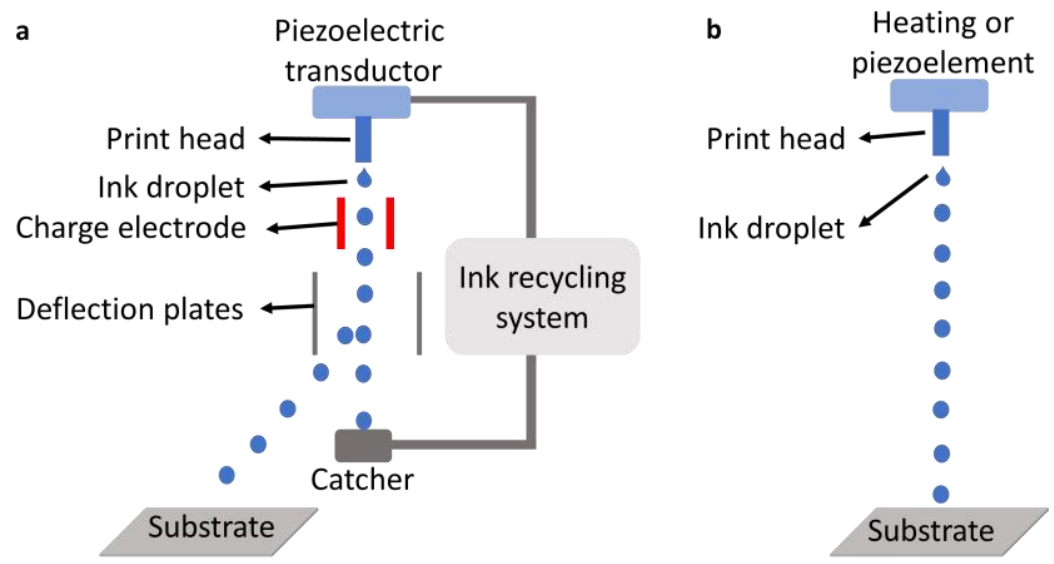

Figure 1.4 Schematic of (a) continuous inkjet and (b) drop-on-demand inkjet printing.

Inkjet printing has several advantages: 1) As a digital printing technique, it does not need a physical mask. Therefore, inkjet printing is highly flexible with respect to pattern design. 2) As a non-contact process, the printer head does not need to contact the substrate physically, which helps to avoid contaminations. 3) Inkjet printing systems can be varied easily from small sized device fabrication systems to large-scale production equipment. However, due to the strict requirements of the inkjet printer, the biggest challenge is to prepare printable inks with proper physical properties like viscosity and surface tension.

Dispersions of nanosheets can be used to prepare printable inks. However, the nanosheet dispersion itself cannot serve directly as an ink. To prepare printable nanosheet-based inks, additives such as surfactants and/or thickeners are added to optimize the physical properties of the inks and improve their storage stability. It has been found that the average lateral sizes and size distributions of nanosheets are important parameters in the preparation of printable ink formulations. For inkjet printed energy storage devices, the average lateral nanosheet size needs to be optimized to get the best electrochemical performance. 
Other key issues in inkjet printing are ink formulation and morphology optimization of inkjet printed patterns, and the avoidance of nozzle clogging. Ink formulation optimization is an efficient way to control droplet formation, and the morphology of printed patterns. Ink surface tension $(\gamma)$ and dynamic viscosity $(\eta)$ are the main two rheological parameters that need to be optimized to get printable and reproducible inks. Inks with surface tensions within the optimum range can be inkjet printed: too low surface tensions would lead to spontaneous ink dripping from the nozzles, while too high values make jetting impossible. ${ }^{22}$ The dynamic viscosity affects the shape, size and velocity of the ejected droplets and is a crucial physical parameter of the ink. ${ }^{23}$ The ideal dynamic viscosity range varies with the type of inkjet printer. Ideally, a Newtonian fluid with a constant viscosity/shear rate relationship is preferred for inkjet printing.

Next to surface tension and dynamic viscosity, another issue in ink formulation concerns the ejection of stable droplets without any satellite droplets or tails that might decrease the resolution of the printed patterns. The droplet jetting behavior can be evaluated by the parameter $Z$, the dimensionless inverse Ohnesorge $(\mathrm{Oh})$ number:

$Z=(\alpha \rho \gamma)^{1 / 2} / \eta$

where $\alpha$ is the nozzle diameter, and $\rho$ is the density of the fluid. By considering single droplet formability, position accuracy and maximum allowable jetting frequency, Jiang et al. demonstrated the optimal range of $Z$ to be between 4 and $14 .{ }^{24}$ However, $Z$ values outside this range have also shown to result in stable jetting behavior. For example, Hsiao et al. reported a $Z$ value as low as 1 for photoresist ink. ${ }^{25}$ Much higher values than 14 are also possible. Stable ink jetting has been reported for ethylene glycol-water ink $(Z=35.5){ }^{26}$ glycerol-water ink $(Z=68.5),{ }^{27}$ as well as for stable water-based inks with a $Z$ value around $19 .{ }^{21}$

The quality of printed patterns influences the performance of printed devices. Non-uniform deposition of solids can lead to a decrease of the resolution of printed patterns and device performance. The undesired coffee-ring effect is a common phenomenon in inkjet printing. The general strategy to prevent it is to modify the physical properties of the ink by adding specific agents. The shape of the suspended particles can also be exploited to optimize the morphology of patterns and reduce the coffee-ring effect. ${ }^{28}$ The morphology of printed patterns must also be optimized in terms of droplet spacing between neighboring droplets and substrate temperature during printing. The ideal droplet spacing for inkjet printing is such that the merging of neighboring droplets does neither lead to overlap, nor to isolated droplets. The morphology of inkjet printed patterns is also affected by the nature of the substrate interface. The wetting of a substrate, which can be defined in terms of contact angle and typically involving terms as hydrophilicity and hydrophobicity, is related to the surface energy and morphology. The wetting process can be described by Young's equation: 
$\gamma_{\mathrm{sv}}=\gamma_{\mathrm{si}}+\gamma_{\mathrm{iv}} \cos \theta$

where $\gamma_{\mathrm{sv}}, \gamma_{\mathrm{si}}$, and $\gamma_{\mathrm{iv}}$ are the interface surface energies between the solid surface (s), the vapor (v) and the ink (i), and $\theta$ is the contact angle. Different contact angles represent different wetting properties. Small contact angles, $\theta<<90^{\circ}$ indicate good wetting of the ink on the substrate, meaning that the ink is able to form a continuous layer. Large angles, $\theta>90^{\circ}$ indicate poor wetting of the ink, meaning that the ink tends to break up into discontinuous patches. However, good wetting with an appropriate contact angle is crucial for functional printing. For instance, surface energy and contact angle need to be optimized carefully to achieve high resolution printed patterns.

Additives like surfactants often need to be added to printable nanosheet-based inks. For example, organic quaternary ammonium ions typically surround nanosheets to prevent their aggregation and precipitation. These additives do not help to improve the electrochemical performance of devices. A post-treatment is thus necessary to improve the electrical properties of printed electrodes. ${ }^{29}$ Thermal annealing is a process in which solvent residues are evaporated and additives are removed.

\subsection{Scope and outline of thesis}

The functionalities of 2D materials offers tremendous opportunities for energy storage applications. By utilizing versatile inkjet printing technology, low-cost and large-scale energy storage devices can be fabricated on different substrates to meet various demands. The research in this thesis is focused on printing different 2D nanosheets as active materials for supercapacitors and Li-ion batteries applications.

In Chapter 2, a printable ink of two-dimensional $\delta-\mathrm{MnO}_{2}$ nanosheets with an average lateral size of $89 \mathrm{~nm}$ and around $1 \mathrm{~nm}$ thickness was prepared in water solution. A small amount of Triton X-100 was added as surfactant to reduce the surface tension of water and propylene glycol was used to increase viscosity of water to meet the requirements of inkjet printer. By optimizing the ink formulation and printing parameters, uniform printed films were achieved without undesired "coffee-ring" effect. Thickness dependent specific capacitance of inkjet printed $\delta-\mathrm{MnO}_{2}$ electrodes were studied. As a proof of concept, all-solid-state symmetrical micro-supercapacitors were fabricated by inkjet printing $\delta-\mathrm{MnO}_{2}$ nanosheets as active materials.

In Chapter 3, defect engineering of $\mathrm{MnO}_{2}$ nanosheets by substitutional doping of $3 d$ metal ions (Co, $\mathrm{Fe}$ and $\mathrm{Ni}$ ) was performed to improve the specific capacitance of $\mathrm{MnO}_{2}$ electrodes. Printable inks of doped $\mathrm{MnO}_{2}$ nanosheets were prepared based on the same ink formulation consisting of Triton X-100 and propylene glycol. The electrochemical performances of doped and pristine printed $\mathrm{MnO}_{2}$ nanosheet electrodes were investigated. First principles calculations were carried out to gain further insight into the effect of aliovalent doping on the 
electronic properties of $\mathrm{MnO}_{2}$ nanosheets. All-solid-state symmetrical micro-supercapacitors were fabricated by printing $\mathrm{Fe}$-doped $\mathrm{MnO}_{2}$ nanosheets as active materials.

In Chapter 4, all-inkjet-printed nanosheets heterostructures were fabricated by printing MXene nanosheets as electrodes and graphene oxide (GO) nanosheets as solid-state electrolyte in sandwiched and interdigitated configurations. Printing parameters were optimized to achieve a clear interface between MXene and GO layers. The printed 2D heterostructures with sandwiched configurations showed high capacitance without any liquid electrolyte present. In contrast, the printed 2D heterostructure with interdigitated configurations only showed comparable capacitance by adding water on top of the devices. The capacitances of both devices could be tuned by adding different liquid electrolytes on top.

In Chapter 5, a 2D heterostructure cathode was fabricated by printing water-based $\mathrm{V}_{2} \mathrm{O}_{5}$ nanosheets and MXene nanosheets composite inks on a current collector to serve as a cathode for Li-ion batteries. MXene nanosheets show excellent electronic conductivity and are highly hydrophilic, which results in good adhesion between printed electrodes and current collectors. Inkjet printing was employed to fabricate electrodes with well controlled surface roughness and precisely controlled thickness. We demonstrate inkjet printed $\mathrm{V}_{2} \mathrm{O}_{5} / \mathrm{Ti}_{3} \mathrm{C}_{2} \mathrm{~T}_{\mathrm{x}}$ thin film cathodes in lithium-ion batteries with high capacity and long cycling life.

In Chapter 6, the crucial challenges and opportunities of printable 2D materials for energy storage devices are discussed.

\subsection{References}

1. Conway, B. E. Electrochemical supercapacitors: scientific fundamentals and technological applications. (Springer Science \& Business Media, 1999).

2. Simon, P., Gogotsi, Y. \& Dunn, B. Where do batteries end and supercapacitors begin? Science 343, 1210-1211 (2014).

3. Whittingham, M. S. Electrical energy storage and intercalation chemistry. Science 192, 1126-1127 (1976).

4. Mizushima, K., Jones, P. C., Wiseman, P. J. \& Goodenough, J. B. $\mathrm{Li}_{\mathrm{x}} \mathrm{CoO}_{2}(0<\mathrm{x}<-$ 1): A new cathode material for batteries of high energy density. Mater. Res. Bull. 15, 783-789 (1980).

5. Yoshino, A., Sanechika, K. \& Nakajima, T. Secondary battery. U. S. Patent No. 4,668,595 (1987). 
6. Whittingham, M. S. \& Yoshino, A. Lithium-ion batteries. (2019). URL https://www.nobelprize.org/uploads/2019/10/advanced-chemistryprize2019.pdf

7. Novoselov, K. S. et al. Electric field effect in atomically thin carbon films. Science 306, 666-669 (2004).

8. Tan, C. et al. Recent advances in ultrathin two-dimensional nanomaterials. Chem. Rev. 117, 6225-6331 (2017).

9. Novoselov, K. S., Mishchenko, A., Carvalho, A. \& Castro Neto, A. H. 2D materials and van der Waals heterostructures. Science 353, aac9439 (2016).

10. Fiori, G. et al. Electronics based on two-dimensional materials. Nat. Nanotechnol. 9, 768-779 (2014).

11. Stoller, M. D., Park, S., Zhu, Y., An, J. \& Ruoff, R. S. Graphene-based ultracapacitors. Nano Letters 8, 3498-3502 (2008).

12. Bonaccorso, F. et al. Graphene, related two-dimensional crystals, and hybrid systems for energy conversion and storage. Science 347 (2015).

13. Zhang, X. \& Xie, Y. Recent advances in free-standing two-dimensional crystals with atomic thickness: design, assembly and transfer strategies. Chem. Soc. Rev. $\mathbf{4 2}$, 8187-8199 (2013).

14. Francesco, B., Antonino, B., N., C. J. \& Claudia, B. 2D-crystal-based functional inks. Adv. Mater. 28, 6136-6166 (2016).

15. Hu, G. et al. Functional inks and printing of two-dimensional materials. Chem. Soc. Rev. 47, 3265-3300 (2018).

16. Li, J., Lemme, M. C. \& Östling, M. Inkjet printing of 2D layered materials. ChemPhysChem 15, 3427-3434 (2014).

17. Kelly, A. G. et al. All-printed thin-film transistors from networks of liquidexfoliated nanosheets. Science 356, 69-73 (2017).

18. Torrisi, F. et al. Inkjet-printed graphene electronics. Acs Nano 6, 2992-3006 (2012).

19. Peng, X. et al. Perovskite and organic solar cells fabricated by inkjet printing: progress and prospects. Adv. Funct. Mater. 27, 1703704 (2017).

20. Zhou, L. et al. Inkjet-printed small-molecule organic light-emitting diodes: halogenfree inks, printing optimization, and large-area patterning. ACS Appl. Mater. Interfaces 9, 40533-40540 (2017). 
21. Wang, Y., Zhang, Y.-Z., Dubbink, D. \& ten Elshof, J. E. Inkjet printing of $\delta-\mathrm{MnO}_{2}$ nanosheets for flexible solid-state micro-supercapacitor. Nano Energy 49, 481-488 (2018).

22. Calvert, P. Inkjet printing for materials and devices. Chem. Mater. 13, 3299-3305 (2001).

23. Reis, N., Ainsley, C. \& Derby, B. Viscosity and acoustic behavior of ceramic suspensions optimized for phase-change ink-jet printing. J. Am. Ceram. Soc. 88, 802-808 (2005).

24. Jang, D., Kim, D. \& Moon, J. Influence of fluid physical properties on ink-jet printability. Langmuir 25, 2629-2635 (2009).

25. Hsiao, W.-K., Hutchings, I., Hoath, S. \& Martin, G. Ink jet printing for direct mask deposition in printed circuit board fabrication. J. Imaging Sci. Techn. 53, 5030450301-50304-50308 (2009).

26. Shin, P., Sung, J. \& Lee, M. H. Control of droplet formation for low viscosity fluid by double waveforms applied to a piezoelectric inkjet nozzle. Microelectron. Reliab. 51, 797-804 (2011).

27. Dong, H. L., Carr, W. W. \& Morris, J. F. An experimental study of drop-on-demand drop formation. Phys. Fluids 18, 072102 (2006).

28. Yunker, P. J., Still, T., Lohr, M. A. \& Yodh, A. G. Suppression of the coffee-ring effect by shape-dependent capillary interactions. Nature 476, 308-311 (2011).

29. ten Elshof, J. E. Electronic band structure and electron transfer properties of twodimensional metal oxide nanosheets and nanosheet films. Curr. Opin. Solid State Mater. Sci. 21, 312-322 (2017). 
Chapter 1 


\section{Chapter 2}

\section{Inkjet Printing of $\delta-\mathrm{MnO}_{2}$ Nanosheets for Flexible Solid-State Micro-Supercapacitor}

Inkjet printing is considered as a promising technique for flexible electronics fabrication owing to its simple, versatile, environmental-friendly and low-cost features. The key to inkjet printing is ink formulation. In this work a highly concentrated ink containing twodimensional $\delta-\mathrm{MnO}_{2}$ nanosheets with an average lateral size of $89 \mathrm{~nm}$ and around $1 \mathrm{~nm}$ thickness was used. By engineering the formulation of the $\delta-\mathrm{MnO}_{2}$ ink, it could be inkjet printed on oxygen plasma treated glass and polyimide film substrates to form $\delta-\mathrm{MnO}_{2}$ patterns without undesired "coffee-ring" effect. As a proof-of-concept application, all-solidstate symmetrical micro-supercapacitors based on $\delta-\mathrm{MnO}_{2}$ nanosheet ink were fabricated. The fabricated MSCs showed excellent mechanical flexibility and good cycling stability with a capacitance retention of $88 \%$ after 3600 charge-discharge cycles. The MSCs attained the highest volumetric capacitance of $2.4 \mathrm{~F} \mathrm{~cm}^{-3}$, and an energy density of $1.8 \cdot 10^{-4} \mathrm{Wh} \mathrm{cm}^{-3}$ at a power density of $0.018 \mathrm{~W} \mathrm{~cm}^{-3}$, which is comparable with other similar devices and show great potential as energy storage units for low-cost flexible and wearable electronics applications.

This chapter has been published as:

Wang, Y.; Zhang, Y.-Z.; Dubbink, D.; ten Elshof, J. E., Inkjet printing of $\delta-\mathrm{MnO}_{2}$ nanosheets for flexible solid-state micro-supercapacitor. Nano Energy 2018, 49, 481-488. 


\subsection{Introduction}

Printed electronics is an emerging technology for flexible electronic device fabrication. ${ }^{1-3}$ Printed devices, including organic transistors, ${ }^{4,5}$ organic light-emitting diodes ${ }^{6}$ and energy storage devices, ${ }^{7-10}$ can be built by printing liquid functional materials such as organic ${ }^{11}$ and inorganic nanomaterials, ${ }^{12}$ as well as two dimensional materials on arbitrary substrates at relatively low temperatures. ${ }^{13}$ Inkjet printing is an ideal method for deposition of nanomaterials for flexible device fabrication because it is a non-contact, precisely controlled deposition and additive printing process.

Owing to their atomically thin layers, high theoretical specific capacitance, environmental compatibility and low cost, birnessite manganese dioxide $\left(\delta-\mathrm{MnO}_{2}\right)$ nanosheets are regarded as an attractive electrode material for portable energy storage devices like supercapacitors. In addition, the layered structure of $\delta-\mathrm{MnO}_{2}$ enables the SCs to be much thinner and flexible than conventional devices. Their fabrication by inkjet printing shows great potential in this respect, since it allows the fabrication of integrated micro-supercapacitors for small size portable, flexible and wearable electronic devices. Alternative methods such as spray coating, ${ }^{14}$ vacuum filtration ${ }^{15}$ and spin coating ${ }^{16}$ have been used to construct MSC devices, but they lack the same degree of control over the roughness of the electrodes and they have limitations in terms of pattern design.

However, a number of challenges still needs to be addressed in order for inkjet printing to become practically feasible. Firstly, ink formulation involving liquid exfoliation processes is far from ideal as it requires multistep processes and is time-consuming. ${ }^{17}$ Secondly, printable ink formulations should have proper fluidic properties, as inkjet printing imposes specific requirements on the physical properties of the ink such as surface tension and viscosity. ${ }^{13}$ Thirdly, the ink should have a high solids concentration and high stability in order to improve the efficiency of the inkjet printing process. ${ }^{18}$

In this study, we developed a highly concentrated, stable, water-based $\delta-\mathrm{MnO}_{2}$ nanosheet ink. No toxic solvents, solvent exchange processes or harsh preparation conditions were required. The $\delta-\mathrm{MnO}_{2}$ ink formulation was optimized for an all-solid-state flexible MSC application.

\subsection{Experimental Section}

\subsubsection{Ink preparation}

Colloidal $\delta-\mathrm{MnO}_{2}$ nanosheets were prepared similar to a previously reported method. ${ }^{19}$ Typically, $20 \mathrm{~mL}$ of a mixed aqueous solution of $0.6 \mathrm{M}$ tetrabutylammonium hydroxide (TBA $\bullet \mathrm{OH}, 40 \mathrm{wt} \% \mathrm{H}_{2} \mathrm{O}$, Alfa Aesar) and $3 \mathrm{wt} \% \mathrm{H}_{2} \mathrm{O}_{2}\left(30 \mathrm{wt} \% \mathrm{H}_{2} \mathrm{O}\right.$, Aldrich) was added to $10 \mathrm{~mL}$ of $0.3 \mathrm{M} \mathrm{MnCl}_{2} \cdot 4 \mathrm{H}_{2} \mathrm{O}$ (Sigma-Aldrich) aqueous solution within $15 \mathrm{~s}$. The resulting dark brown solution was stirred vigorously overnight in the ambient at room temperature. The solution obtained was centrifuged using a Sigma 1-14 centrifuge at $1000 \mathrm{~g}$ for 20 minutes 
before collecting the upper $2 / 3$ of the volume. The lower $1 / 3$ was washed by water and methanol at $295 \mathrm{~g}$ for 20 minutes, after which the precipitate was dried at room temperature. The collected supernatant was centrifuged at $15000 \mathrm{~g}$ for $1 \mathrm{~h}$ and the precipitate was redispersed in the printing solvent. The printing solvent consisted of 1:10 propylene glycol (Sigma-Aldrich): water by mass, $0.06 \mathrm{mg} \mathrm{mL}^{-1}$ Triton X-100 (Sigma-Aldrich). Then the redispersion solution was filtered through a $0.2 \mu \mathrm{m}$ syringe filter to remove large flakes which might block the ink jet printer nozzles.

In order to estimate the final concentration of $\delta-\mathrm{MnO}_{2}$ in the above ink, $100 \mu \mathrm{L}$ ink was diluted in water by 500 times on volume. The optical absorbance was measured using a Shimadzu UV-1800 UV-Vis spectrophotometer at 800-300 nm wavelength. According to the Lambert-Beer law $A / l=\alpha C$, where $A$ is the absorbance, $l$ the cell length (here $l=1 \mathrm{~cm}$ ), $C$ the concentration of dispersed $\delta-\mathrm{MnO}_{2}$ and the absorption coefficient $\alpha=1.13 \times 10^{4} \mathrm{~mol}^{-1}$ $\mathrm{dm}^{3} \mathrm{~cm}^{-1}$ for $\delta-\mathrm{MnO}_{2}$ nanosheets at around $374 \mathrm{~nm},{ }^{20}$ the $\delta-\mathrm{MnO}_{2}$ concentrations $C$ in the ink was estimated to be $8.8 \mathrm{mg} \mathrm{mL}^{-1}$.

Poly(3,4-ethylenedioxythiophene): poly(styrenesulfonate) (PEDOT: PSS, 3.0-4.0\%, Sigma Aldrich) solution was filtered through a $0.45 \mu \mathrm{m}$ syringe filter followed by addition of $2 \mathrm{vol} \%$ Triton X-100 and 6 vol\% ethylene glycol (Merck).

\subsubsection{Printing}

All patterns and devices were inkjet printed by a Dimatix DMP-2800 inkjet printer (Fujifilm Dimatix) which equipped with a $10 \mathrm{pL}$ cartridge (DMC-11610). Our formulated $\delta-\mathrm{MnO}_{2}$ ink was printed on different substrates, including glass and $120 \mu \mathrm{m}$ thick polyimide film substrates. PEDOT: PSS ink was inkjet printed on top of printed $\delta-\mathrm{MnO}_{2}$ film at a drop spacing of $20 \mu \mathrm{m}$ at room temperature. The substrates, including glass and polyimide film, were cleaned by ethanol, acetone, isopropanol and water followed by $\mathrm{O}_{2}$ plasma treatment for $5 \mathrm{~min}$.

\subsubsection{Fabrication of MSC}

First, $\delta-\mathrm{MnO}_{2}$ ink was inkjet printed in 5 layers at $20 \mu \mathrm{m}$ drop spacing on a $120 \mu \mathrm{m}$ thick polyimide substrate, followed by annealing at $350{ }^{\circ} \mathrm{C}$ for $1 \mathrm{~h}$ under nitrogen atmosphere. Then, 2 layers of PEDOT: PSS were inkjet printed at $20 \mu \mathrm{m}$ drop spacing on top of the thermally treated $\delta-\mathrm{MnO}_{2}$ thin films, followed by thermal annealing at $120{ }^{\circ} \mathrm{C}$ for $15 \mathrm{~min}$. The prepared PEDOT: PSS $/ \delta-\mathrm{MnO}_{2}$ films were used as electrodes to for a symmetrical MSC. The PVA/LiCl gel electrolyte was prepared by mixing $1 \mathrm{~g}$ PVA ( $M_{\mathrm{W}} 85000-124000$, Aldrich), $2.13 \mathrm{~g} \mathrm{LiCl}$ (Alfa Aesar) and $10 \mathrm{~mL}$ DI water thoroughly at $85{ }^{\circ} \mathrm{C}$ under vigorous stirring. To complete the MSC, the electrolyte was deposited on the electrodes area of MSC and was dried at room temperature overnight.

\subsubsection{Electrochemical testing}


All electrochemical characterization was done by an Autolab workstation (PGSTAT128N). The prepared PEDOT: $\mathrm{PSS} / \delta-\mathrm{MnO}_{2}$ electrode was tested using a three-electrode configuration in $0.5 \mathrm{M} \mathrm{Na}_{2} \mathrm{SO}_{4}(\mathrm{ABCR} \mathrm{GmbH})$ solution. A platinum wire and an $\mathrm{Ag} / \mathrm{AgCl}$ $(3 \mathrm{M} \mathrm{KCl})$ electrode $(\mathrm{Metrohm})$ were used as the counter and reference electrodes, respectively. The electrochemical performance of the all-solid-state MSC was measured in a two-electrode configuration. Cyclic voltammetry (CV) curves were obtained at a scan rate of 5 to $100 \mathrm{mV} \mathrm{s}^{-1}$, galvanostatic charge-discharge (GCD) curves were measured at current densities from 0.05 to $0.2 \mathrm{~A} \mathrm{~cm}^{-3}$. Electrochemical impedance spectroscopy was performed by applying an $\mathrm{AC}$ voltage of $10 \mathrm{mV}$ amplitude in the frequency range from 0.01 to $10 \mathrm{kHz}$.

\subsubsection{Characterization}

X-ray diffraction (XRD) was conducted on a PANalytical X'Pert Pro with $\mathrm{Cu} \mathrm{K} \alpha$ radiation $(\lambda=0.15405 \mathrm{~nm})$. High resolution scanning electron microscopy (HRSEM; Zeiss MERLIN) was used to acquire information on the morphology of printed $\delta-\mathrm{MnO}_{2}$ nanosheets films. Atomic force microscopy (AFM, Veeco Dimension Icon) was performed in standard tapping mode. The AFM data were analyzed by Gwyddion (version 2.47) software. X-ray photoelectron spectroscopy (XPS) was measured by an Omicron Nanotechnology GmbH (Oxford Instruments) surface analysis system with a photon energy of $1486.7 \mathrm{eV}$ (Al Ka Xray source) with a scanning step size of $0.1 \mathrm{eV}$. The pass energy was set to $20 \mathrm{eV}$. The spectra were corrected using the binding energy of $\mathrm{C} 1 s$ of the adventitious carbon as a reference. Transmission electron microscopy (TEM) was performed by FEI Titan 80-300 ST (300 kV) with energy dispersive X-ray spectroscopy (EDS) capabilities. UV-vis spectra were measured by a UV-1800 Shimadzu. The surface tension of the ink was measured by contact angle system OCA (Data Physics Corporation). The viscosity was determined by an Automated Microviscometer AMVn (Anton Paar $\mathrm{GmbH}$ ). The specific volumetric capacitance $\left(C_{\mathrm{V}}\right)$ of film electrodes was calculated from the GCD curves by using Equation (1):

$C_{\mathrm{V}}=[I /(\mathrm{d} V / \mathrm{d} t)] / V_{\text {electrode }}$

where $I$ is the discharge current, $\mathrm{d} V / \mathrm{d} t$ is the slope of discharge curve, and $V_{\text {electrode }}$ refers to the volume of the film electrode.

The specific areal capacitance $\left(C_{\mathrm{A} \text {,device }}\right)$ and volumetric capacitance $\left(C_{\mathrm{V} \text {,device }}\right)$ of the MSC devices were also calculated from the GCD curve according to equations (2) and (3), respectively:

$$
\begin{aligned}
& C_{\mathrm{A}, \text { device }}=[I /(\mathrm{d} V / \mathrm{d} t)] / A_{\text {device }} \\
& C_{\mathrm{V}, \text { device }}=[I /(\mathrm{d} V / \mathrm{d} t)] / V_{\text {device }}
\end{aligned}
$$


Here $A_{\text {device }}$ refers to the total area of the device including the electrodes and the gap between the electrodes. $V_{\text {device }}$ refers to the total volume of the device, including the volume of the electrodes and the gap between the electrodes.

The volumetric energy densities $\left(E_{\mathrm{V}}, \mathrm{Wh} \mathrm{cm}^{-3}\right)$ and power densities $\left(P \mathrm{~V}, \mathrm{~W} \mathrm{~cm}{ }^{-3}\right)$ were calculated from equations (4) and (5)

$E_{\mathrm{V}}=C_{\mathrm{V}, \text { device }} V^{2} /(2 \times 3600)$

$P_{\mathrm{V}}=3600 \times E_{\mathrm{V}} / \Delta t$

Where $\Delta t$ refers to discharge time.

\subsection{Results and discussion}

a
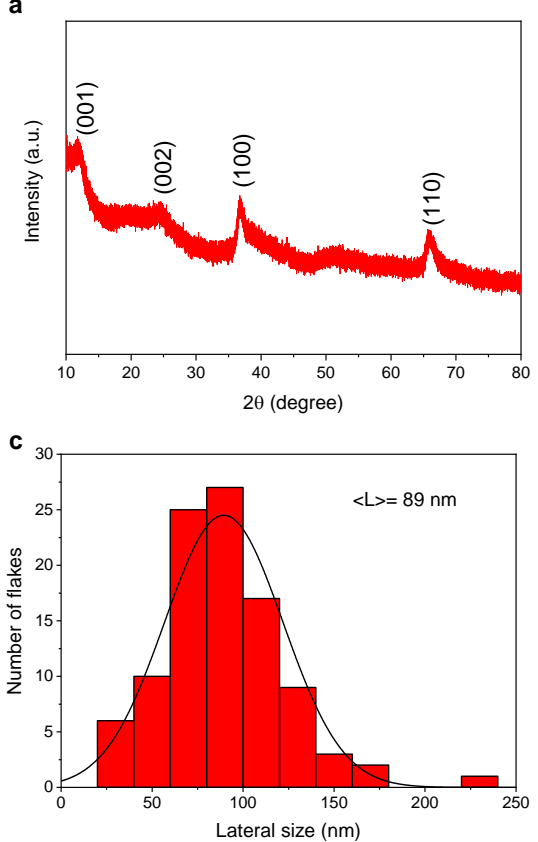

b

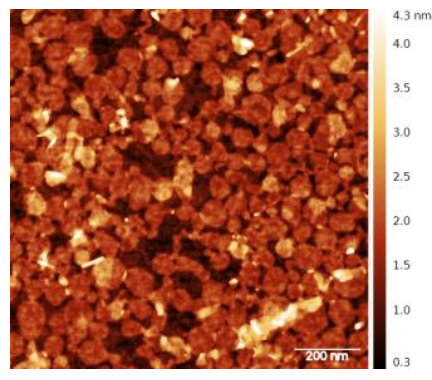

d

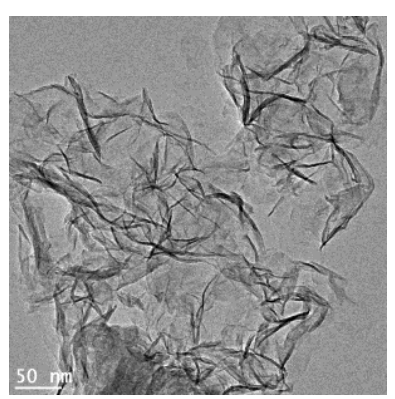

Figure 2.1 Characterization of $\delta-\mathrm{MnO}_{2}$ nanosheets. (a) XRD pattern of $\delta-\mathrm{MnO}_{2}$ nanosheets. (b) AFM image of $\delta$ $\mathrm{MnO}_{2}$ nanosheets after deposition on a Si wafer by LB technology. (c) Lateral size distribution of $\delta$ - $\mathrm{MnO}_{2}$ nanosheets obtained by measuring 100 nanosheet flakes in Figure 1b. (d) TEM image of $\delta-\mathrm{MnO}_{2}$ nanosheets.

Powder XRD of a dried sample of a colloidal suspension after centrifuging and washing with distilled water and methanol, was used to verify the crystal structure and phase information of the $\delta-\mathrm{MnO}_{2}$ nanosheets as shown in Figure 2.1a. The XRD pattern shows the characteristic peaks at $2 \theta 12.21^{\circ}, 24.55^{\circ}, 36.71^{\circ}, 65.87^{\circ}$, which are attributable to the (001), (002), (100) 
and (110) reflections. ${ }^{21}$ These peaks indicate a layered birnessite-type structure. The thickness of a $\delta-\mathrm{MnO}_{2}$ nanosheet deposited on a silicon substrate by Langmuir-Blodgett (LB) technology was measured by AFM and was around $1 \mathrm{~nm}$ (Figure S2.1, Appendices). Based on its atomic architecture, the crystallographic thickness of monolayer $\delta-\mathrm{MnO}_{2}$ nanosheets has been calculated to be $0.52 \mathrm{~nm} .{ }^{19}$ Hydration and the presence of organic ions, i.e. tetrabutylammonium $\left(\mathrm{TBA}^{+}\right)$, on both sides of the $\delta-\mathrm{MnO}_{2}$ nanosheets can explain the difference between the crystallographic thickness and the observed thickness. ${ }^{19}$ The lateral sizes of $\delta-\mathrm{MnO}_{2}$ nanosheets estimated from AFM images (Figure 2.1b) indicate that the majority of nanosheets has lateral sizes between 50 and $150 \mathrm{~nm}$ (Figure 2.1c), which meets the requirement of the inkjet printer. In principle, a lateral size of less than $1 / 50$ the diameter of nozzle is preferred to avoid the nozzle from becoming clogged during printing. ${ }^{22}$ Based on this rule of thumb, the maximum nanosheet lateral size is around $430 \mathrm{~nm}$ for our inkjet printer with a nozzle diameter of $21.5 \mu \mathrm{m}$. Figure 2.1d shows a TEM image of $\delta-\mathrm{MnO}_{2}$ nanosheets, illustrating the ultrathin nature of the 2D nanostructure.
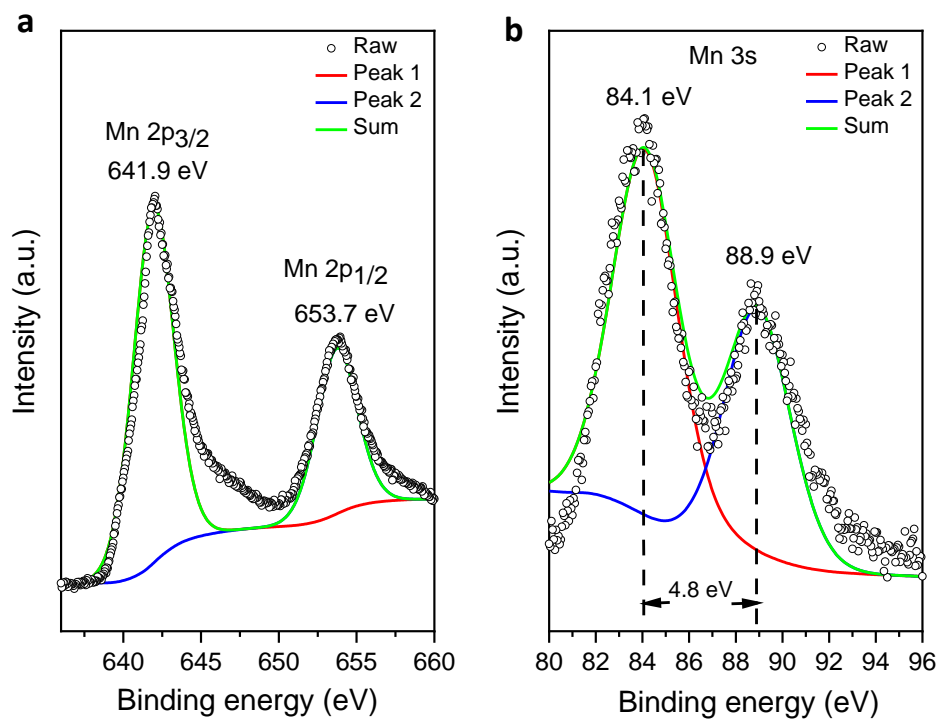

Figure 2.2 High resolution XPS spectra of (a) Mn $2 p$ and (b) $\mathrm{Mn} 3 s$ of $\delta-\mathrm{MnO}_{2}$ nanosheets.

XPS was used to determine the oxidation state of $\mathrm{Mn}$ in $\delta-\mathrm{MnO}_{2}$ nanosheets. The two peaks at the binding energies of $641.9 \mathrm{eV}$ and $653.7 \mathrm{eV}$ as shown in Figure 2.2a, can be assigned to the $\mathrm{Mn} 2 p_{3 / 2}$ and $2 p_{1 / 2}$ orbitals of $\mathrm{Mn}^{4+}$, respectively. The Mn $3 s$ spectrum displays double peaks that result from parallel spin coupling between the electrons in $3 s$ and $3 d$ orbitals, with a splitting width of $4.8 \mathrm{eV}$, further indicating that the Mn cations have an average valence close to 4 (Figure $2.2 \mathrm{~b}){ }^{23}$ 
a

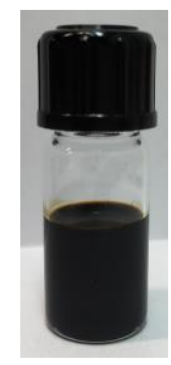

b

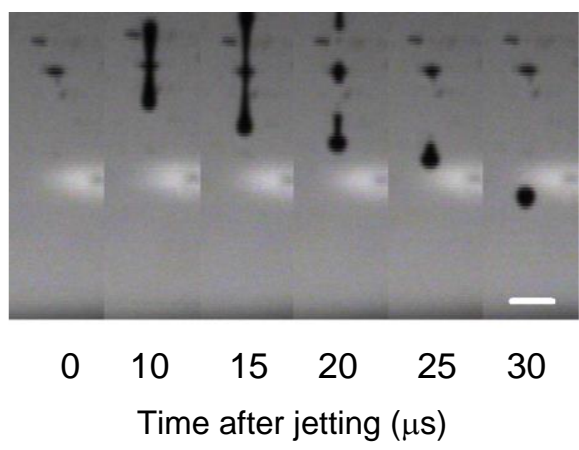

d

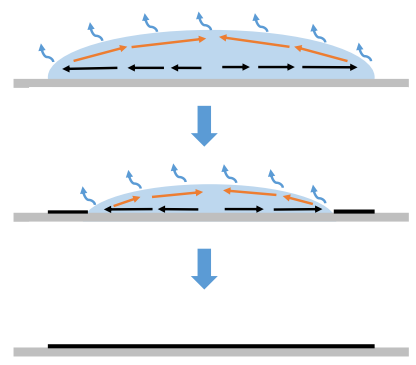

C

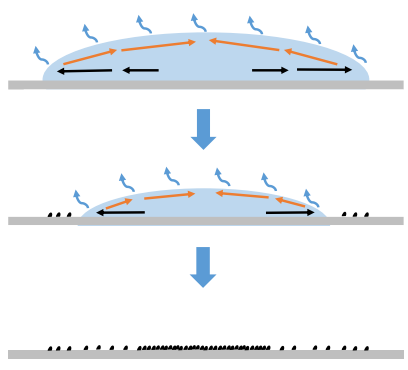

$\longrightarrow$ Outward flow $\longrightarrow$ Marangoni flow Solvent evaporation

Figure 2.3 Optimization of $\delta-\mathrm{MnO}_{2}$ ink formulation. (a) Photograph of formulated $\delta-\mathrm{MnO}_{2}$ nanosheet ink. (b) Optical image of $\delta-\mathrm{MnO}_{2}$ ink droplet formation vs time as observed from the printer camera. The scale bar is $50 \mu \mathrm{m}$. Droplet drying process with (c) excess surfactant and (d) optimal surfactant concentration.

Water as such is not suitable for inkjet printing due to its high surface tension (about $70 \mathrm{mN}$ $\mathrm{m}^{-1}$ ) and low viscosity (about $1 \mathrm{mPa} \mathrm{s}$ ). The inverse Ohnesorge number $Z$ is often used to evaluate ink printability, and is defined as $Z=(\gamma \rho \alpha)^{1 / 2} / \eta$, where $\gamma$ is the surface tension, $\rho$ the density, $\alpha$ the nozzle diameter and $\eta$ the viscosity of the fluid. To formulate a printable $\delta$ $\mathrm{MnO}_{2}$ ink (Figure 2.3a), Triton X-100, a non-ionic surfactant, was selected as surface tension modifier to decrease the surface tension of water from around 73 to $46 \mathrm{mN} \mathrm{m}^{-1}$. Triton may also help to avoid disrupting the electrostatic stabilization of $\delta-\mathrm{MnO}_{2}$ nanosheets. Propylene glycol was added to modify the viscosity from 1.00 to $1.71 \mathrm{mPa}$ s in order to improve printing reliability. The value of the surface tension, viscosity and nozzle diameter of $21.5 \mu \mathrm{m}$ makes that $Z$ is about 19 for the modified water-based ink. This quality of the ink was confirmed by the optical images of ink droplet formation vs time where no satellite droplets are present (Figure 2.3b). An additional advantage of the addition of propylene glycol is that it can also suppress weak Marangoni flow which will reduce the undesired coffee-ring effect. ${ }^{24}$ 


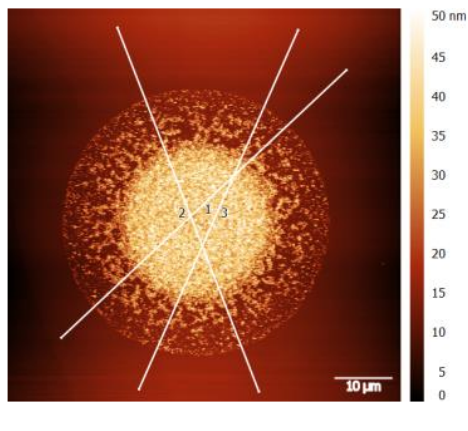

C

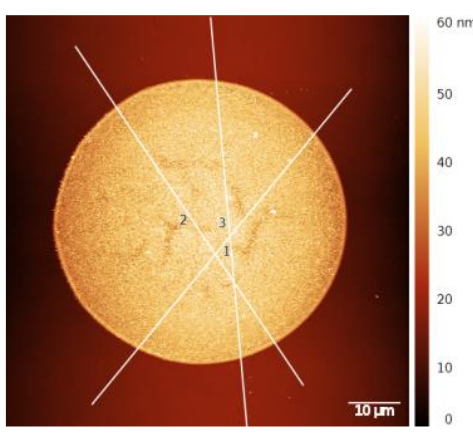

b

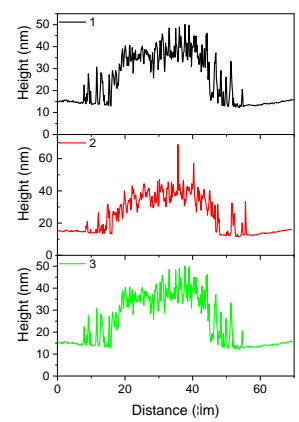

d

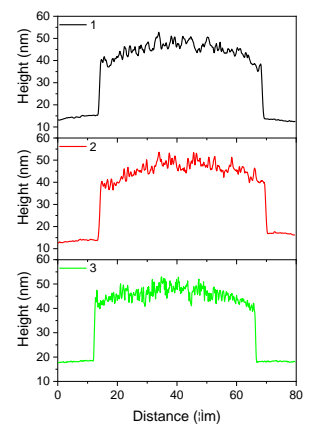

Figure 2.4 (a) AFM image of printed single dot on glass substrate with excess surfactant. (b) Cross-sectional profiles along three different directions in (a). (c) AFM image of printed single dot on glass substrate with optimized surfactant concentration. (d) Cross-sectional profiles along three different directions in (c).

The concentration of Triton X-100 was optimized since an excess tends to shrink the droplet size. As schematically outlined in Figure 2.3c, excess Triton X-100 unpins the contact line led to a non-uniform distribution of solids, which can indeed be clearly observed by AFM (Figure 2.4a). The cross-sectional profile of the AFM image of Figure 2.4a in Figure 2.4b further confirms the pattern non-uniformity. More AFM images and cross-sectional profiles along different directions of non-uniform printed line are shown in Figure S2.2a and S2.2b (Appendices). The concentration of Triton X-100 was therefore carefully optimized to ensure the pinning of the contact line of the ink. Under ideal conditions the material is uniformly deposited on the substrate due to recirculating Marangoni flow, as schematically shown in Figure $2.3 \mathrm{~d}$. The AFM image in Figure 2.4c shows a printed dot obtained from an ink with an optimized Triton X-100 concentration. The corresponding cross-sectional analysis in Figure $2.4 \mathrm{~d}$ reveals pattern uniformity in all directions, indicating the reliability and quality of the printing process. The printed patterns also show a smooth surface and low root mean square roughness at higher magnification, as shown in Figure S2.3 (Appendices). 


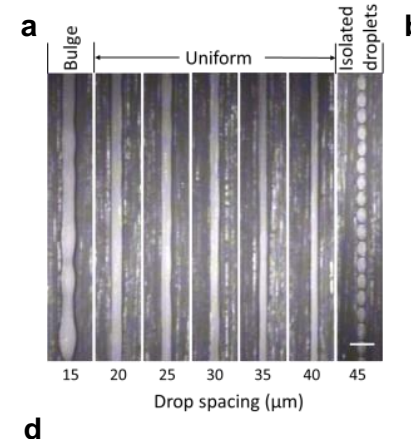

d

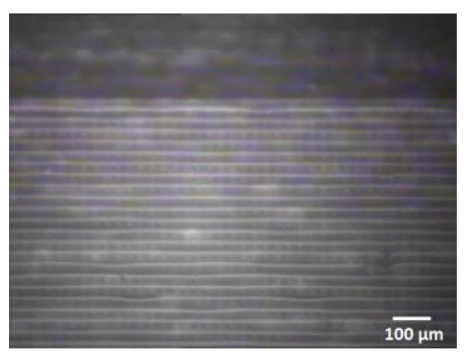

b

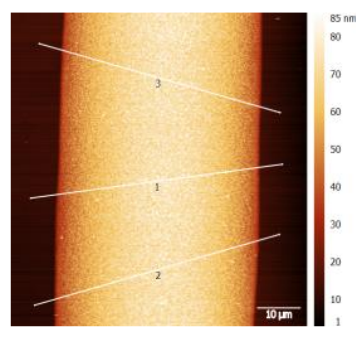

e
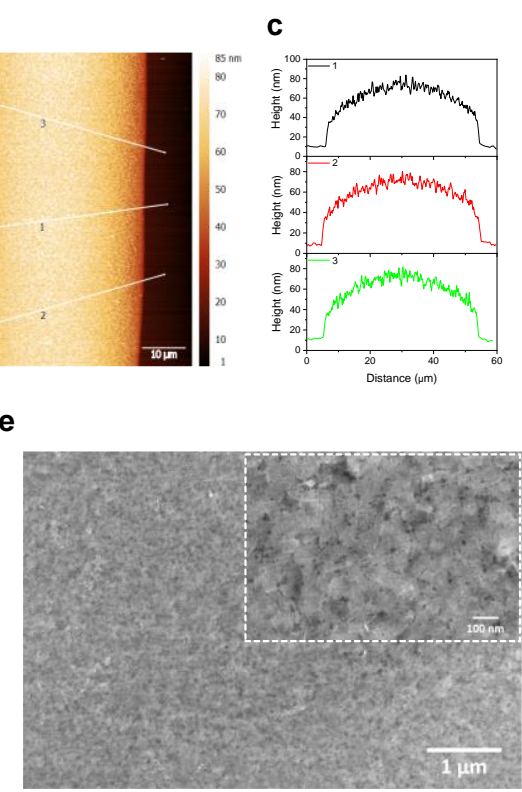

Figure 2.5 Optimization of $\delta-\mathrm{MnO}_{2}$ ink printing parameters. (a) Optical images of printed lines at different droplet spacings. The scale bar is $100 \mu \mathrm{m}$. (b) AFM image of printed line at $40 \mu \mathrm{m}$ drop spacing. (c) Cross sectional profiles along three different directions in (b). (d) Optical image of printed $\delta-\mathrm{MnO}_{2}$ thin films on glass substrate. (e) Topview SEM images of (d) at different magnifications, in which the $\delta-\mathrm{MnO}_{2}$ nanosheets are uniformly distributed.

The morphology of printed $\delta-\mathrm{MnO}_{2}$ lines on glass substrate at $50{ }^{\circ} \mathrm{C}$ with variable droplet spacing is shown in Figure 2.5a. The line became bulged when the droplet spacing was 15 $\mu \mathrm{m}$, due to the fact that droplets significantly overlap with each other at this spacing. As the droplet spacing increased to $40 \mu \mathrm{m}$, the morphology of the lines became more uniform while the line width decreased. Any further increase of the droplet spacing led to isolated droplets as they were too far from each other to merge. The homogeneous morphology and fidelity of printed lines employing a $40 \mu \mathrm{m}$ droplet spacing was confirmed by AFM; Figure $2.5 \mathrm{~b}$ shows a uniform distribution of nanosheet, while the cross-sectional profiles of Figure $2.5 \mathrm{~b}$ in Figure $2.5 \mathrm{c}$ confirm the uniformity of the printed lines in all directions. The morphology of printed $\delta-\mathrm{MnO}_{2}$ lines on polyimide substrate at room temperature with variable droplet spacing was also studied as shown in Figure S2.4 (Appendices). The lines became uniform using a droplet spacing from 20 to $50 \mu \mathrm{m}$. In order to reduce printing layers and improve printing efficiency, a droplet spacing at $20 \mu \mathrm{m}$ was used for printing $\delta-\mathrm{MnO}_{2}$ ink on polyimide substrate. The $\delta$ $\mathrm{MnO}_{2}$ ink was also used to print thin films with uniformly distributed $\delta-\mathrm{MnO}_{2}$ nanosheets, as illustrated in Figure $2.5 \mathrm{~d}$ where the optical image of a printed $\delta-\mathrm{MnO}_{2}$ film on a glass substrate is shown, and Figure 2.5e where the top-view SEM images of Figure $2.5 \mathrm{~d}$ at different magnifications are shown. 
a

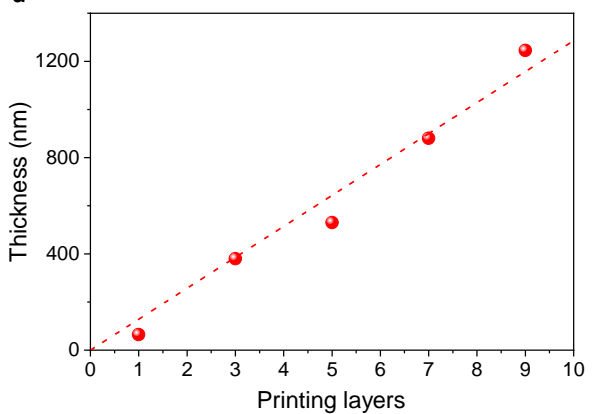

c

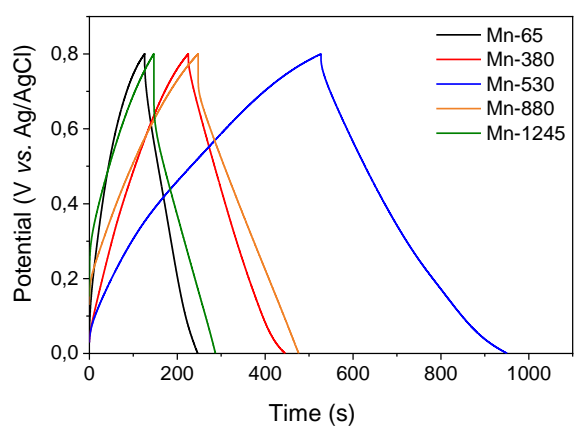

b

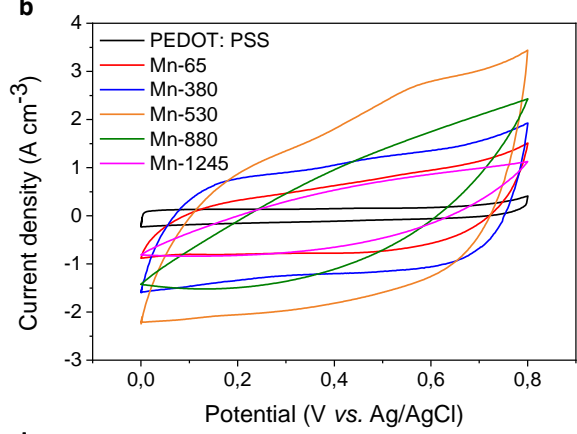

d

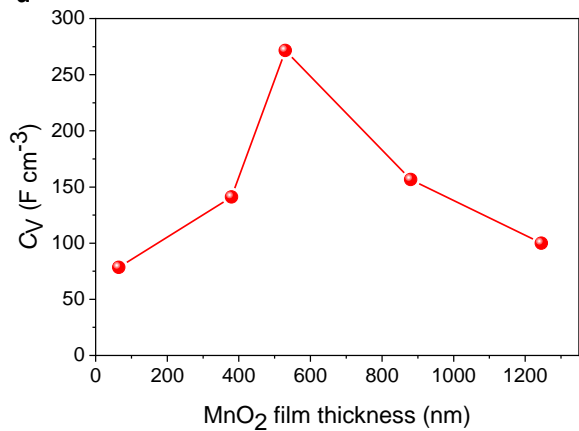

Figure 2.6 Electrochemical performance of printed $\delta-\mathrm{MnO}_{2}$ films with varying thickness. (a) Relationship between $\delta-\mathrm{MnO}_{2}$ film thickness and the number of printed layers. (b) $\mathrm{CV}$ curves of $\delta-\mathrm{MnO}_{2}$ films with varying thicknesses at a scan rate of $10 \mathrm{mV} \mathrm{s}^{-1}$. (c) GCD of $\delta-\mathrm{MnO}_{2}$ electrodes with varying thicknesses at a current density of $0.5 \mathrm{~A} \mathrm{~cm}$ ${ }^{3}$. (d) Volumetric capacitances $C_{\mathrm{V}}$ of $\delta-\mathrm{MnO}_{2}$ electrodes as a function of film thickness at $0.5 \mathrm{~A} \mathrm{~cm}^{-3}$.

To investigate the electrochemical performance of a printed $\delta-\mathrm{MnO}_{2}$ film, printed PEDOT: $\mathrm{PSS} / \delta-\mathrm{MnO}_{2}$ electrodes on polyimide substrates were made and studied in three-electrode measurements. The reliable printing process allowed us to print multilayered $\delta-\mathrm{MnO}_{2}$ films with different $\delta-\mathrm{MnO}_{2}$ film thicknesses. As shown in Figure 2.6a, the thickness of these printed $\delta-\mathrm{MnO}_{2}$ films was proportional to the number of printed layers. A series of electrodes with varying $\delta-\mathrm{MnO}_{2}$ films thicknesses between 65 and $1245 \mathrm{~nm}$ were made. These electrodes are referred to as Mn-65, Mn-380, Mn-530, Mn-880, and Mn-1245, depending on their thickness (in nanometers). All electrodes were characterized in a three-electrode setup in $0.5 \mathrm{M} \mathrm{Na}_{2} \mathrm{SO}_{4}$ solution. The $\mathrm{CV}$ curves of these electrodes at a scan rate of $10 \mathrm{mV} \mathrm{s}^{-1}$ show rectangular-like shapes (Figure 2.6b), which are explained by the redox reaction $\mathrm{MnO}_{2}+\mathrm{Na}^{+}$ $+\mathrm{e}^{-} \rightleftarrows$ MnOONa. The GCD curves in Figure 2.6c were acquired at a current density of 0.5 $\mathrm{A} \mathrm{cm}^{-3}$. The calculated volumetric capacitances $\left(C_{\mathrm{V}}\right)$ are shown in Figure $2.6 \mathrm{~d}$. As the thickness of $\delta-\mathrm{MnO}_{2}$ films increased to $65 \mathrm{~nm}$, the $C_{\mathrm{V}}$ of the $\mathrm{Mn}-65$ electrode reached 78.4 $\mathrm{F} \mathrm{cm}^{-3}$, which is higher than the pure PEDOT: PSS electrode $\left(23.4 \mathrm{~F} \mathrm{~cm}^{-3}\right)$. The maximum $C_{\mathrm{V}}$ of $271.6 \mathrm{~F} \mathrm{~cm}^{-3}$ was obtained with the Mn-530 electrode, and this value is about an order 
of magnitude higher than the pure PEDOT: PSS film. This value is also clearly higher than the $C_{\mathrm{V}}$ of the $65 \mathrm{~nm}$ thick film, showing that the $\delta-\mathrm{MnO}_{2}$ nanosheet layers contribute to the electrode reaction. In contrast, when the thickness of the $\delta-\mathrm{MnO}_{2}$ film was further increased to $880 \mathrm{~nm}$, the $C_{\mathrm{V}}$ of the $\mathrm{Mn}-880$ electrode decreased dramatically to $156.6 \mathrm{~F} \mathrm{~cm}^{-3}$. Most likely, electron transfer between layers becomes limiting in thick $\delta-\mathrm{MnO}_{2}$ film, probably to the extent that the $\mathrm{MnO}_{2}$ nanosheet layers of the electrode furthest away from the external electrode are electrically isolated and do not contribute to the capacitance of the supercapacitor. Slow electron transfer kinetics or electrical insulation between adjacent nanosheet layers has been observed in various studies involving multilayers of nanosheets. ${ }^{25}$ The $C_{\mathrm{V}}$ of the even thicker Mn-1245 electrode decreased further to $100 \mathrm{~F} \mathrm{~cm}^{-3}$. Possibly, the electrically insulating top part of the electrode acts only as a diffusion barrier for $\mathrm{Na}^{+}$. In any case these results clearly show that the optimum thickness of the MSC is in the range of about $500 \mathrm{~nm}$.

a
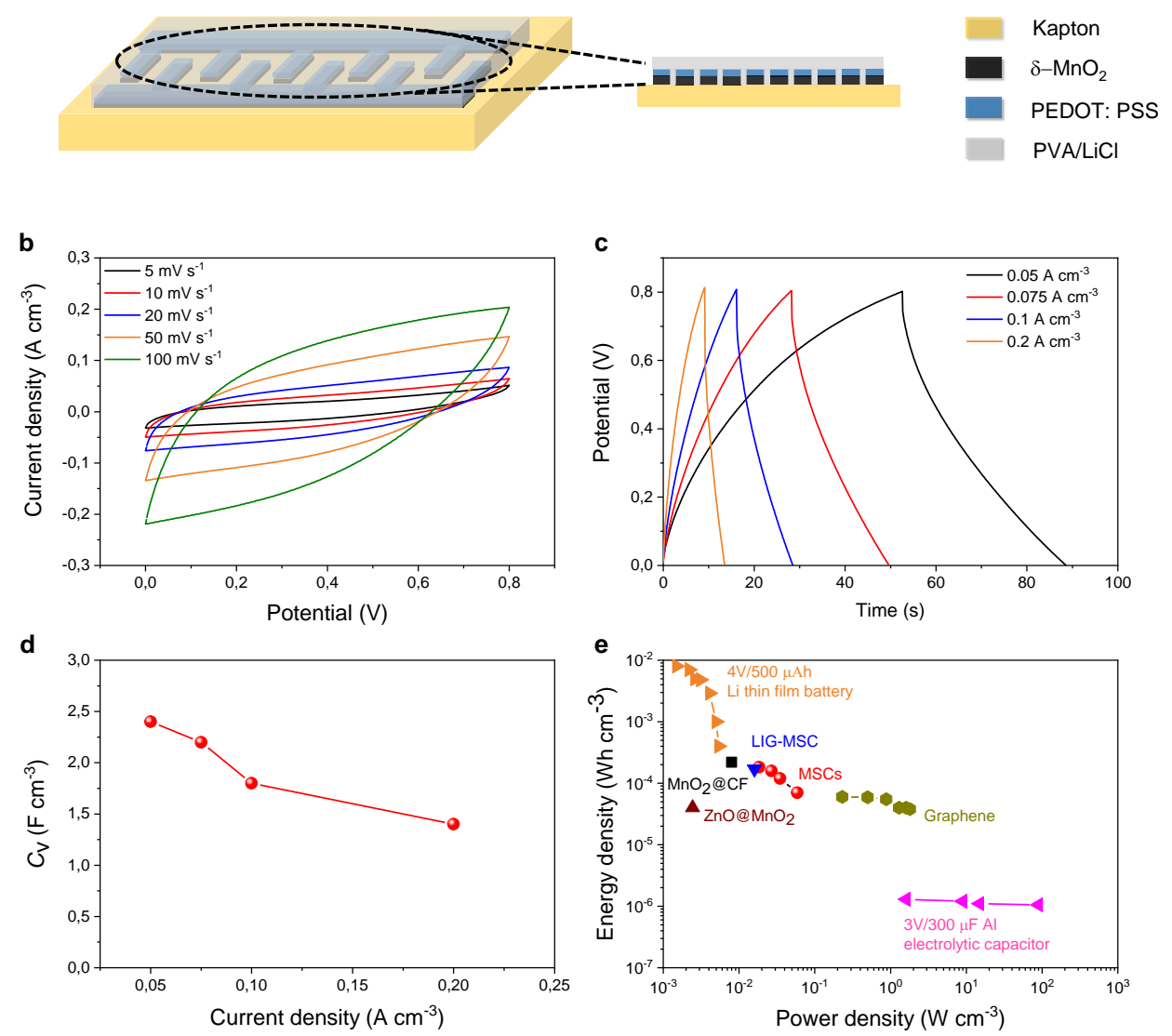

Figure 2.7 Electrochemical performance of inkjet printed MSC. (a) Schematic diagram of MSC with interdigitated electrode configuration. (b) $C V$ curves of MSC at scan rates from 5 to $100 \mathrm{mV} \mathrm{s}^{-1}$. (c) Galvanostatic charge-discharge 
curves of MSC at current densities from 0.05 to $0.2 \mathrm{~A} \mathrm{~cm}^{-3}$. (d) Volumetric capacitance of MSC at different current densities. (e) Ragone plot of MSC and recent data from literatures..$^{26-31}$

To further investigate the use of $\delta-\mathrm{MnO}_{2}$ nanosheets for practical application, a symmetrical MSC with interdigitated electrode configuration was fabricated using inkjet printing $\delta-\mathrm{MnO}_{2}$ on a flexible polyimide substrate, as schematically illustrated in Figure S2.5 (Appendices). Functional $\delta-\mathrm{MnO}_{2}$ based devices including 10 in-plane interdigitated patterns were printed. The $\delta-\mathrm{MnO}_{2}$ film was about $530 \mathrm{~nm}$ thick, as shown in the SEM image of the cross-section of the film in Figure S2.6 (Appendices). After drying the $\delta-\mathrm{MnO}_{2}$ pattern, PEDOT: PSS conducting electrodes were inkjet printed on top of the $\delta-\mathrm{MnO}_{2}$ patterns. Then a poly(vinyl alcohol)/ lithium chloride $(\mathrm{PVA} / \mathrm{LiCl})$ gel electrolyte was cast onto the surface of the PEDOT: PSS $/ \delta-\mathrm{MnO}_{2}$ electrode to complete the fabrication of the MSC (Figure 2.7a). In order to evaluate the electrochemical performance of the MSC, CV and galvanostatic chargedischarge measurements were carried out in a potential window from 0 to $0.8 \mathrm{~V}$. The $\mathrm{CV}$ curves of the MSC at different scan rates showed a rectangular-like shape at low scan rates, which was maintained at high scan rates up to $100 \mathrm{mV} \mathrm{s}^{-1}$ (Figure 2.7b). The charge-discharge curves are shown in Figure 2.7c. The volumetric capacitance of the MSC was calculated based on the charge-discharge measurements. As shown in Figure 2.7d, the MSC showed a highest volumetric device capacitance of $2.4 \mathrm{~F} \mathrm{~cm}^{-3}$ at a current density of $0.05 \mathrm{~A} \mathrm{~cm}^{-3}$. This value corresponds with an areal capacitance of $0.26 \mathrm{mF} \mathrm{cm}$. The areal capacitance is comparable to most graphene-based MSCs fabricated by other techniques, ${ }^{32,33}$ and can be used in many on-chip integrated systems which only require areal capacitances of around 1 $\mu \mathrm{F} \mathrm{cm}^{-2}{ }^{26,27}$ Notably, the volume used in the calculation of the volumetric capacitance included the volume of the electrodes and the spatial gap between the electrodes, while the area used in the calculation of the areal capacitance includes both the electrode area and the area of the gap between the electrodes. Figure 2.7e shows Ragone plots of the volumetric energy density and the power density of the MSC, as well as a comparison with other recently reported SC systems. The high equivalent series resistance (ESR) of the MSC indicates a low charge/discharge rate (see Figure S2.7, Appendices). The energy density for the MSC is evaluated to be $1.8 \times 10^{-4} \mathrm{Wh} \mathrm{cm}^{-3}$, with a power density of $0.018 \mathrm{~W} \mathrm{~cm}^{-3}$. Hence, the energy density of the nanosheet-based inkjet printed MSC is superior to a commercial $3 \mathrm{~V} / 300 \mu \mathrm{F}$ $\mathrm{Al}$ electrolyte capacitor, ${ }^{28}$ as well as to other supercapacitors such as $\mathrm{ZnO} @ \mathrm{MnO}_{2}$ carbon fiber ${ }^{29}$ and graphene. ${ }^{30}$ The performance of the nanosheet-based MSC is comparable to other devices made of $\mathrm{MnO}_{2} /$ carbon fibers ${ }^{31}$ and laser-induced graphene (LIG) MSC. ${ }^{34}$ 
a
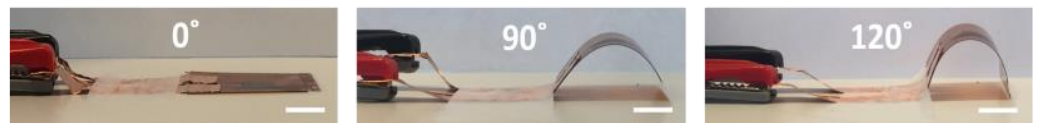

b

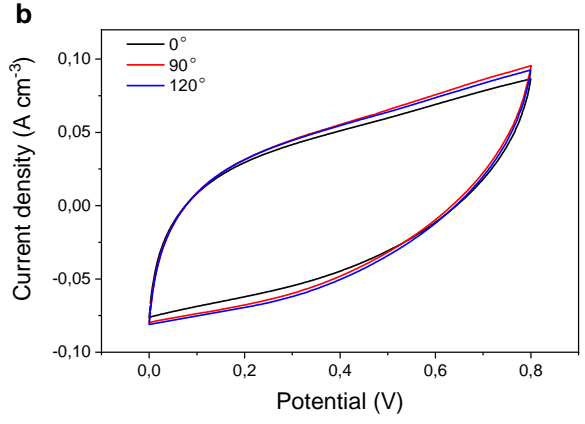

d

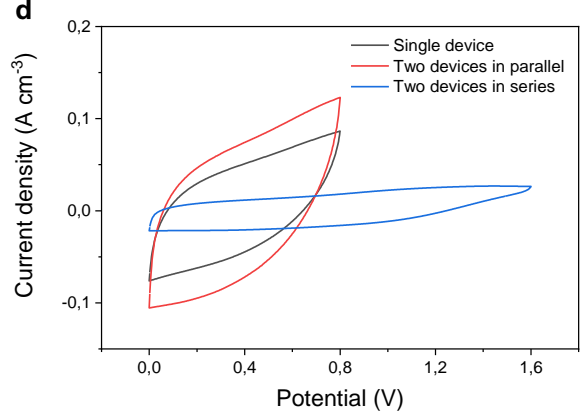

C
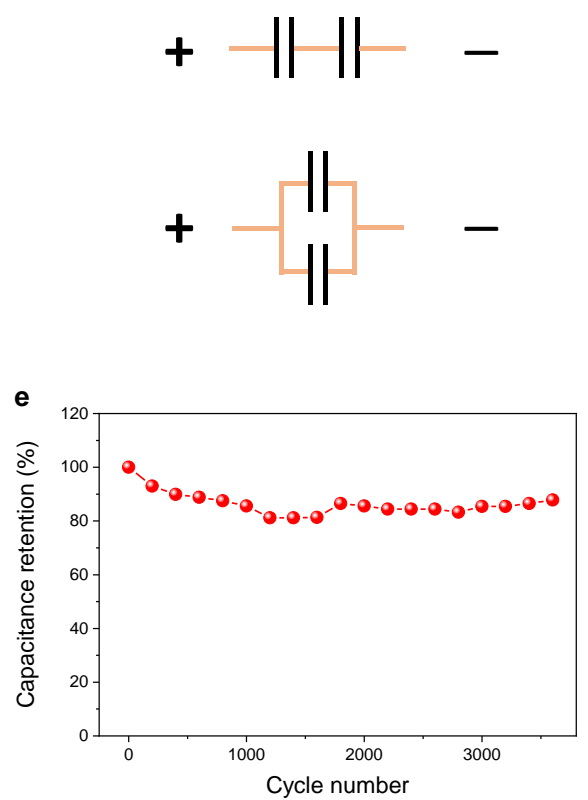

Figure 2.8 Flexibility and cycling measurement, as well as assembly of two MSC devices in series and parallel configurations. (a) Optical images of MSC bent under different angles. The scale bar is $1 \mathrm{~cm}$. (b) CV curves of MSC under different bending angles at a scan rate of $20 \mathrm{mV} \mathrm{s}^{-1}$. (c) Schematic circuits of two single MSC devices connected in series and in parallel configurations. (d) CV curves of single MSC, and two MSCs connected in series or in parallel. (e) Cyclability test of MSC at a current density of $0.2 \mathrm{~A} \mathrm{~cm}^{-3}$.

To demonstrate the mechanical flexibility of the MSC, the device was bent at different angles (Figure 2.8a). The CV curves remained nearly unchanged while the device was highly bent over $120^{\circ}$ with a bending radius of about $1 \mathrm{~cm}$ (Figure $2.8 \mathrm{~b}$ ), indicating that the MSC has potential as energy storage unit cell for small flexible electronics applications. Furthermore, the device was also bent for 250 times with a bending radius of about $1 \mathrm{~cm}$. As shown in Figure S2.8 (Appendices), the CV curves showed a slight decrease after 100 times bending and a further decrease after 250 times bending due to the occurrence of a small crack in the electrode (Figure S2.9, Appendices). However, the device was still functional, albeit operating at a lower performance. To meet the requirements for practical application to satisfy specific energy and power needs, MSCs can be connected in series or parallel configurations (Figure 2.8c). The voltage window was doubled by connecting two MSCs in 
series, while the output current was increased by a factor of almost 2 when two MSCs were connected in parallel (Figure 2.8d), indicating that these devices can be integrated to scale up the voltage and current output. A $22 \%$ drop in volumetric capacitance of MSC over 3600 charge-discharge cycles was observed (Figure 2.8e), indicating good cycling stability. It is noted that this work focused on demonstrating the efficiency and possibility of inkjet printing technology for realizing flexible $\delta-\mathrm{MnO}_{2}$ nanosheet-based MSC devices. We did not attempt to determine the performance limits of these devices. Devices performance improvements may be expected by integrating other fabrication strategies with our inkjet printing technology, such as chemical doping of $\delta-\mathrm{MnO}_{2}$ nanosheets in order to improve conductivity and/or energy density.

\subsection{Conclusions}

We have developed water-based, inkjet printable and highly concentrated $\delta-\mathrm{MnO}_{2}$ nanosheets inks for supercapacitor application. By ink formulation engineering, examining the drop spacing, we determined the optimal printing conditions to prevent the undesired "coffee-ring" effect. We have shown that the inkjet printed MSCs are mechanically flexible and achieve high performance, which is comparable with other MSCs fabricated by different techniques. The inkjet printing of two-dimensional materials also shows a high potential for all-solidstate flexible energy storage devices. Overall, such inkjet printed flexible energy storage devices shows great promising as energy storage units for low-cost flexible and wearable electronics applications.

\subsection{References}

1. Ahn, J.-H. et al. Heterogeneous three-dimensional electronics by use of printed semiconductor nanomaterials. Science 314, 1754-1757 (2006).

2. Cao, Q. et al. Medium-scale carbon nanotube thin-film integrated circuits on flexible plastic substrates. Nature 454, 495 (2008).

3. Ahn, B. Y. et al. Omnidirectional printing of flexible, stretchable, and spanning silver microelectrodes. Science 323, 1590-1593 (2009).

4. Sekitani, T. et al. A large-area wireless power-transmission sheet using printed organic transistors and plastic MEMS switches. Nat. Mater. 6, 413-417 (2007).

5. Noh, Y.-Y., Zhao, N., Caironi, M. \& Sirringhaus, H. Downscaling of self-aligned, all-printed polymer thin-film transistors. Nat. Nanotechnol. 2, 784-789 (2007).

6. Zheng, H. et al. All-solution processed polymer light-emitting diode displays. Nat. Commun. 4, 1971 (2013). 
7. Gaikwad, A. M., Steingart, D. A., Ng, T. N., Schwartz, D. E. \& Whiting, G. L. A flexible high potential printed battery for powering printed electronics. Appl. Phys. Lett. 102, 233302 (2013).

8. Hu, L., Wu, H. \& Cui, Y. Printed energy storage devices by integration of electrodes and separators into single sheets of paper. Appl. Phys. Lett. 96, 183502 (2010).

9. Pang, H., Zhang, Y., Lai, W.-Y., Hu, Z. \& Huang, W. Lamellar $\mathrm{K}_{2} \mathrm{Co}_{3}\left(\mathrm{P}_{2} \mathrm{O}_{7}\right)_{2} \cdot 2 \mathrm{H}_{2} \mathrm{O}$ nanocrystal whiskers: high-performance flexible all-solid-state asymmetric microsupercapacitors via inkjet printing. Nano Energy 15, 303-312 (2015).

10. Huang, M. et al. Synthesis of $\mathrm{Co}_{3} \mathrm{O}_{4} / \mathrm{SnO}_{2} @ \mathrm{MnO}_{2}$ core-shell nanostructures for high-performance supercapacitors. J. Mater. Chem. A 3, 12852-12857 (2015).

11. Berggren, M., Nilsson, D. \& Robinson, N. D. Organic materials for printed electronics. Nat. Mater. 6, 3 (2007).

12. $\mathrm{Wu}, \mathrm{W}$. Inorganic nanomaterials for printed electronics: a review. Nanoscale 9, 7342-7372 (2017).

13. Bonaccorso, F., Bartolotta, A., Coleman, J. N. \& Backes, C. 2D-crystal-based functional inks. Adv. Mater. 28, 6136-6166 (2016).

14. Wu, Z.-S., Liu, Z., Parvez, K., Feng, X. \& Müllen, K. Ultrathin printable graphene supercapacitors with AC line-filtering performance. Adv. Mater. 27, 3669-3675 (2015).

15. Xiao, H. et al. One-step device fabrication of phosphorene and graphene interdigital micro-supercapacitors with high energy density. ACS Nano 11, 7284-7292 (2017).

16. Cheng, T., Zhang, Y.-Z., Zhang, J.-D., Lai, W.-Y. \& Huang, W. High-performance free-standing PEDOT:PSS electrodes for flexible and transparent all-solid-state supercapacitors. J. Mater. Chem. A 4, 10493-10499 (2016).

17. Nicolosi, V., Chhowalla, M., Kanatzidis, M. G., Strano, M. S. \& Coleman, J. N. Liquid exfoliation of layered materials. Science 340, 1226419 (2013).

18. Qian, J. et al. Aqueous manganese dioxide ink for paper-based capacitive energy storage devices. Angew. Chem. Int. Ed. 54, 6800-6803 (2015).

19. Kai, K. et al. Room-temperature synthesis of manganese oxide monosheets. J. Am. Chem. Soc. 130, 15938-15943 (2008). 
20. Omomo, Y., Sasaki, T., Wang \& Watanabe, M. Redoxable nanosheet crystallites of $\mathrm{MnO}_{2}$ derived via delamination of a layered manganese oxide. J. Am. Chem. Soc. 125, 3568-3575 (2003).

21. Lee, S.-W. et al. Structural changes in reduced graphene oxide upon $\mathrm{MnO}_{2}$ deposition by the redox reaction between carbon and permanganate ions. J. Phys. Chem. C 118, 2834-2843 (2014).

22. Hutchings, I. M. \& Martin, G. D. Inkjet technology for digital fabrication. (John Wiley \& Sons, 2012).

23. Biesinger, M. C. et al. Resolving surface chemical states in XPS analysis of first row transition metals, oxides and hydroxides: Cr, Mn, Fe, Co and Ni. Appl. Surf. Sci. 257, 2717-2730 (2011).

24. McManus, D. et al. Water-based and biocompatible 2D crystal inks for all-inkjetprinted heterostructures. Nat. Nanotechnol. 12, 343-350 (2017).

25. ten Elshof, J. E. Electronic band structure and electron transfer properties of twodimensional metal oxide nanosheets and nanosheet films. Curr. Opin. Solid State Mater. Sci. 21, 312-322 (2017).

26. Thekkekara, L. V. et al. On-chip energy storage integrated with solar cells using a laser scribed graphene oxide film. Appl. Phys. Lett. 107, 031105 (2015).

27. Westover, A. S. et al. Direct integration of a supercapacitor into the backside of a silicon photovoltaic device. Appl. Phys. Lett. 104, 213905 (2014).

28. Pech, D. et al. Ultrahigh-power micrometre-sized supercapacitors based on onionlike carbon. Nat. Nanotechnol. 5, 651-654 (2010).

29. Yang, P. et al. Hydrogenated $\mathrm{ZnO}$ core-shell nanocables for flexible supercapacitors and self-powered systems. ACS Nano 7, 2617-2626 (2013

30. El-Kady, M. F., Strong, V., Dubin, S. \& Kaner, R. B. Laser scribing of highperformance and flexible graphene-based electrochemical capacitors. Science $\mathbf{3 3 5}$, 1326-1330 (2012).

31. Xiao, X. et al. Fiber-based all-solid-state flexible supercapacitors for self-powered systems. ACS Nano 6, 9200-9206 (2012).

32. Gao, W. et al. Direct laser writing of micro-supercapacitors on hydrated graphite oxide films. Nat. Nanotechnol. 6, 496-500 (2011). 
33. Wu, Z. S., Parvez, K., Feng, X. \& Müllen, K. Graphene-based in-plane microsupercapacitors with high power and energy densities. Nat. Commun. 4, 2487 (2013).

34. Peng, Z. et al. Flexible boron-doped laser-induced graphene microsupercapacitors. ACS Nano 9, 5868-5875 (2015). 


\section{APPENDICES}

\section{Fabrication of Langmuir-Blodgett (LB) thin film}

The lateral size of nanosheets is a crucial parameter for inkjet printing. Lateral size analysis of $\delta-\mathrm{MnO}_{2}$ nanosheets was performed by AFM in standard tapping mode. $\delta-\mathrm{MnO}_{2}$ nanosheets were deposited on a silicon substrate by LB technology. In LB deposition, $1 \mathrm{~mL}$ of $\delta-\mathrm{MnO}_{2}$ ink was diluted in $500 \mathrm{~mL}$ DI water. The diluted suspension was left standing for $2 \mathrm{~h}$, then $50 \mathrm{~mL}$ of solution was taken from the middle or top part of the nanosheet suspension and poured into an LB trough (KSV Minimicro, a Teflon trough with an active trough surface area of $100 \mathrm{~cm}^{2}, \mathrm{~L} 195 \times \mathrm{W} 51 \times \mathrm{D} 4 \mathrm{~mm}^{3}$ and a dipping well $\mathrm{L} 10 \times \mathrm{W} 28 \times \mathrm{D} 28 \mathrm{~mm}^{3}$, trough volume $48 \mathrm{~cm}^{3}$ ) and left for $15 \mathrm{~min}$ to equilibrate and stabilize the surface pressure before the LB deposition process started. Prior to deposition, the silicon substrate was first cleaned by acetone, ethanol and DI water. Then the cleaned silicon substrate was placed in Harrick Plasma PDC-002 oxygen plasma cleaner $(25 \mathrm{~W})$ for $15 \mathrm{~min}$ to remove any residual organic on the silicon surface. Then silicon substrate was immersed vertically into the suspension. Film formation and transfer was conducted by starting compression at a rate of $3 \mathrm{~mm} \mathrm{~min} \mathrm{~m}^{-1}$ by moving the Teflon barriers until a threshold surface pressure had been reached. The $\delta$ $\mathrm{MnO}_{2}$ nanosheets films were deposited at $7 \mathrm{mN} \mathrm{m}^{-1}$ surface pressure as measured by a Wilhelmy plate attached to the KSV Minimicro frame.
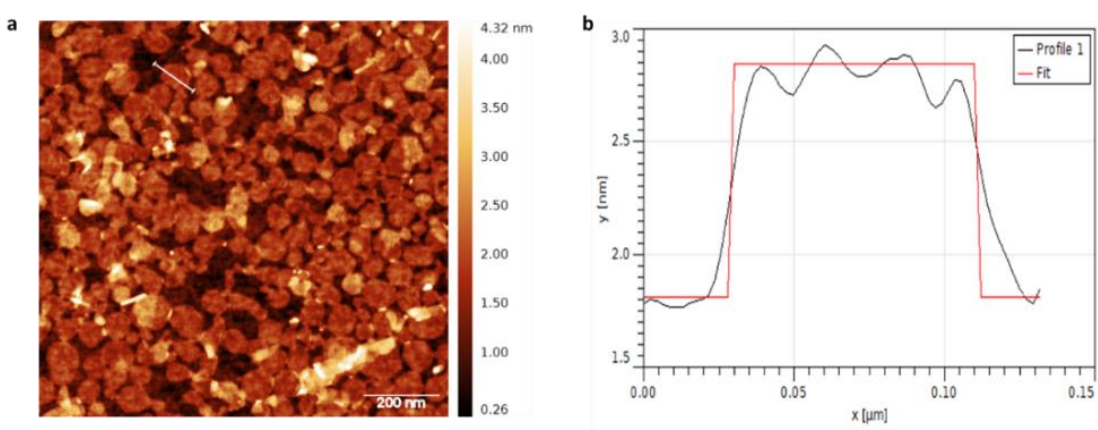

Figure S2.1 (a) AFM images and (b) height profile of $\delta-\mathrm{MnO}_{2}$ nanosheets. 

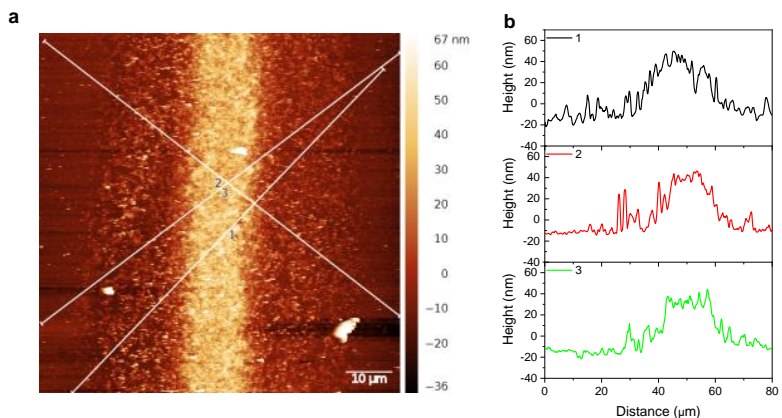

Figure S2.2 (a) AFM image of printed line on glass with excess surfactant. (b) Cross sectional profiles along three different directions in (a).

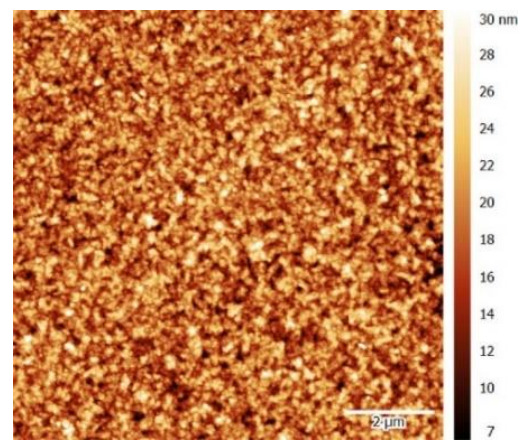

Figure S2.3 AFM image of printed single dot. The mean root square roughness is $3.7 \pm 0.3 \mathrm{~nm}$.

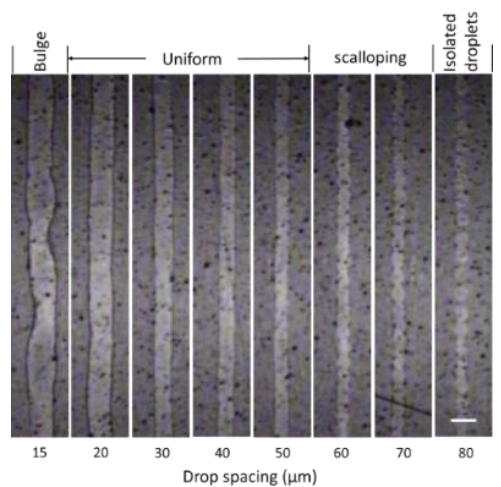

Figure S2.4 Optical images of printed $\delta$ - $\mathrm{MnO}_{2}$ lines at different droplet spacing on polyimide substrate. The scale bar is $100 \mu \mathrm{m}$. 


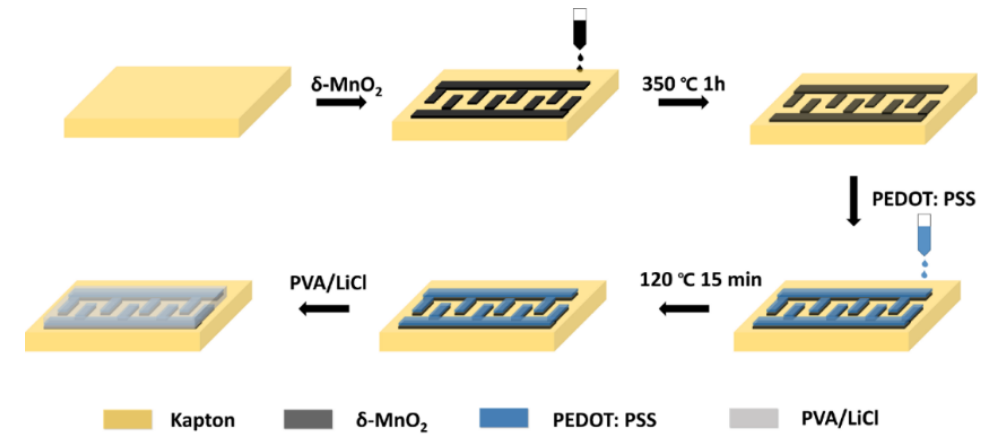

Figure S2.5 Schematic illustration of fabrication process of interdigitated MSC.

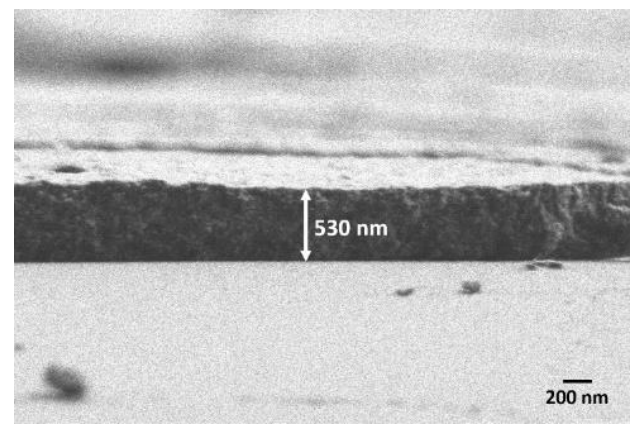

Figure S2.6 SEM image of printed $\delta-\mathrm{MnO}_{2}$ film on polyimide substrate.

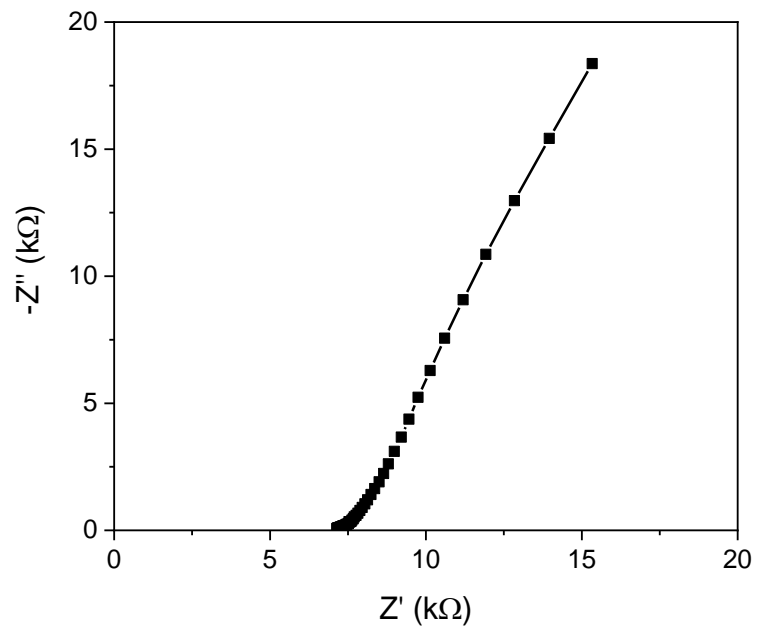

Figure S2.7 EIS curve of MSC for the frequency range from 0.1 to $10 \mathrm{kHz}$. 


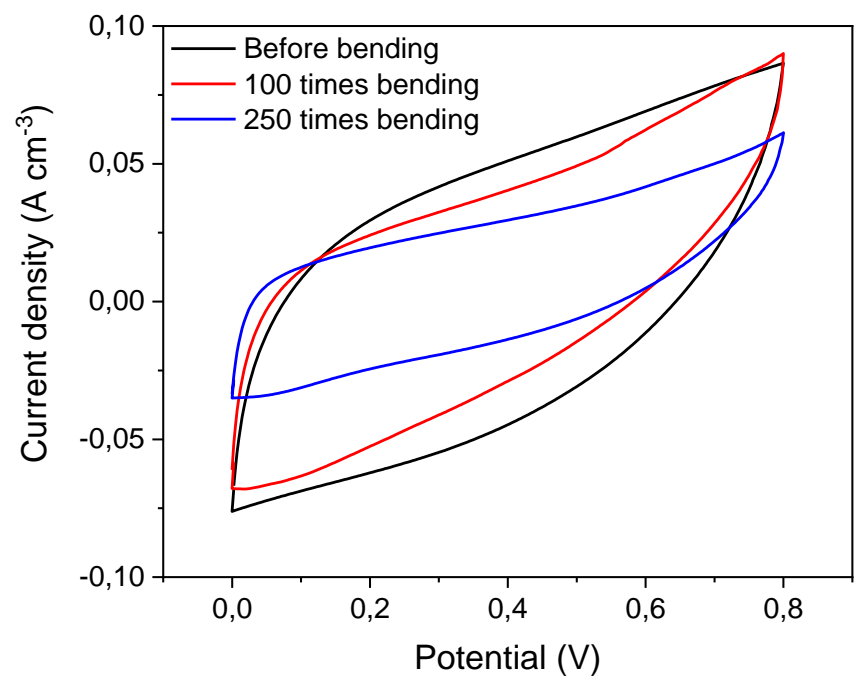

Figure S2.8 CV curves of MSC under different bending times with bending radius of about $1 \mathrm{~cm}$ at a scan rate of $20 \mathrm{mV} \mathrm{s}^{-1}$.
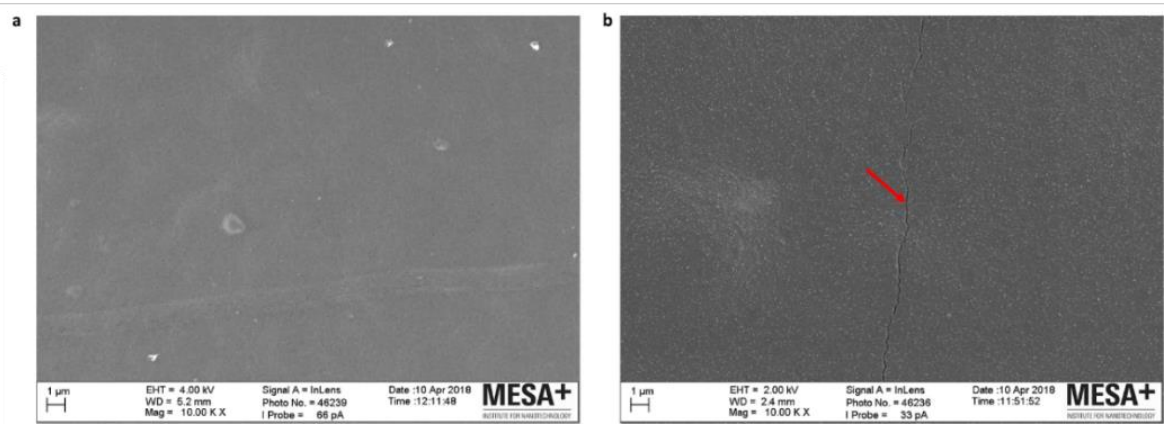

Figure S2.9 SEM images of electrodes after (a) 100 times and (b) 250 times bending with bending radius of about $1 \mathrm{~cm}$. 
Chapter 2 


\section{Defect Engineering of $\mathrm{MnO}_{2}$ Nanosheets by Substitutional Doping for Printable Solid-State Micro-Supercapacitors}

Printed flexible energy storage devices such as micro-supercapacitors require high electrochemical performance for practical applications. Here, we report a high volumetric energy density of up to $1.13 \times 10^{-3} \mathrm{Wh} \mathrm{cm}^{-3}$ at a power density of $0.11 \mathrm{~W} \mathrm{~cm}^{-3}$ by inkjet printing of Fe-doped $\mathrm{MnO}_{2}$ nanosheets inks as active materials on polyimide substrates. The enhancement results from atomic-level substitutional doping of $3 d$ metal ions (Co, Fe, $\mathrm{Ni}$ ) in sub-nanometer thick $2 \mathrm{D} \mathrm{MnO}_{2}$ nanosheets. Substitutional doping introduces new electronic states near the Fermi level, thereby enhancing the electronic conductivity and contributing to the formation of redox-active $3 d$ surface states. Fe-doped $\mathrm{MnO}_{2}$ showed the best performance in terms of specific areal and volumetric capacitance. Our finding suggests that the rational doping at atomic scale shows great promise for achieving high energy and power density flexible energy storage devices.

This chapter has been published as:

Wang, Y.; Zhang, Y.-Z.; Gao, Y.-Q.; Sheng, G.; ten Elshof, J. E., Defect engineering of $\mathrm{MnO}_{2}$ nanosheets by substitutional doping for printable solid-state micro-supercapacitors. Nano Energy 2020, 68, 104306. 


\subsection{Introduction}

Two-dimensional (2D) materials have attracted great attention for supercapacitors (SCs) because of their unique physical and chemical properties induced by the dimensional reduction. ${ }^{1-3}$ Specifically, 2D nanosheets with a few atomic-layer thickness and large specific surface area can expose nearly all its atoms as surface sites for charge storage, which facilitates the ion diffusion and the charge storage and transfer processes. Recently, various nanosheets have been explored for SCs, including graphene, ${ }^{4}$ black phosphorus, ${ }^{5}$ metal dichalcogenides, ${ }^{6}$ layer double hydroxides, ${ }^{1}$ transition metal carbides, ${ }^{7}$ and transition metal oxides (TMOs). ${ }^{8,9}$ Among these, TMOs are known as pseudocapacitive phases with high theoretical specific capacitance, and are thus promising electrode materials for SCs. From the TMO phases, 2D manganese dioxide nanosheets have been attracting interest due to the earth abundance of $\mathrm{Mn}$, low toxicity and particularly their ultrathin structure, ${ }^{10,11}$ and research on $\mathrm{MnO}_{2}$ based SCs has been progressing since the late 1990s. ${ }^{12,13}$ However, the performance of $\mathrm{MnO}_{2}$ nanosheets in SCs is limited by poor electronic conductivity. One of the strategies to improve the electronic conductivity and electrochemical performance of $\mathrm{MnO}_{2}$ electrodes is to combine it with highly conductive materials such as graphene, carbon nanotubes or carbon fibers. ${ }^{14}$ An alternative strategy is defect engineering, i.e. foreign element doping to enhance the intrinsic electronic conductivity and increase the concentration of redox-active sites. ${ }^{15-17}$ Thus far, defect engineering has not resulted in notable improvement in electrochemical performance. Transition metal cations like $\mathrm{Fe}^{3+}, \mathrm{Co}^{2+}$ and $\mathrm{Ni}^{2+}$ are known to be stable in the framework of birnessite, ${ }^{18-20}$ but their effect on the electronic conductivity of $\mathrm{MnO}_{2}$ nanosheets is still unknown.

Micro-supercapacitors (MSCs) which feature rapid power delivery, on-chip integration and miniaturized device size, are promising energy storage devices for small flexible electronic devices. ${ }^{21-24}$ Versatile fabrication techniques have been utilized to directly deposit materials on different substrates to fabricate interdigitated electrode patterns for MSCs, such as laser scribing, electrochemical deposition, conversion reaction and inkjet printing. ${ }^{25}$ While the laser scribing technique suffers from high cost, the electrochemical deposition and conversion reactions are restricted by the shapes of current collectors. Inkjet printing, which is a digital, non-contact, and high resolution deposition technique, does not require an intermediate carrier for deposition on a wide range of substrates such as silicon, glass, paper or flexible polymers. ${ }^{26-29}$ However, preparation of printable functional inks remain a significant challenge for printed electronics.

In this work, we report inkjet-printed $2 \mathrm{D} \mathrm{MnO}_{2}$ nanosheet-based micro-supercapacitors with high volumetric energy densities of up to $2.47 \times 10^{-3} \mathrm{Wh} \mathrm{cm}^{-3}$ at a power density of $4.16 \times$ $10^{-2} \mathrm{~W} \mathrm{~cm}^{-3}$. The excellent performance could be accomplished by atomic-level substitutional doping of $3 d$ metal ions ( $\mathrm{Co}, \mathrm{Fe}, \mathrm{Ni}$ ) into $2 \mathrm{D} \mathrm{MnO}_{2}$ nanosheets by a facile bottom-up method, which introduced new electronic states near the Fermi level, thereby 
enhancing the electronic conductivity within the nanosheets and contributing to the formation of redox-active $3 d$ surface states. Stable water-based inks were prepared without toxic solvents and were based on the surfactant-templated self-assembly of $\mathrm{MnO}_{2}$ nanosheets in water. Three-electrode measurements were conducted on inkjet-printed undoped $\mathrm{MnO}_{2}$, and on Fe, Co and $\mathrm{Ni}$-doped $\mathrm{MnO}_{2}$ nanosheet thin films. The influence of substitutional doping on band structure and the excellent performance of Fe doping in particular was explained using first principles calculations, which demonstrates that substitutional doping can largely improve the electrochemical performance of $2 \mathrm{D}$ oxide materials.

\subsection{Experimental Section}

\subsubsection{Synthesis of Fe, Co and $\mathrm{Ni}$-doped $\mathrm{MnO}_{2}$ nanosheets}

In a typical synthesis, $20 \mathrm{~mL}$ of a mixed aqueous solution of $0.6 \mathrm{M}$ tetrabutylammonium hydroxide (TBA $\bullet \mathrm{OH}, 40 \mathrm{wt} \% \mathrm{H}_{2} \mathrm{O}$, Alfa Aesar) and $3 \mathrm{wt} \% \mathrm{H}_{2} \mathrm{O}_{2}\left(30 \mathrm{wt} \% \mathrm{H}_{2} \mathrm{O}\right.$, Aldrich) was added to $10 \mathrm{~mL}$ of $0.3 \mathrm{M}$ aqueous solution consisting of $\mathrm{FeCl}_{3}$ (Fluka) and $\mathrm{MnCl}_{2} \bullet 4 \mathrm{H}_{2} \mathrm{O}$ (Sigma-Aldrich) in a molar ratio of $0.05\left(0.05=\mathrm{Fe} /(\mathrm{Fe}+\mathrm{Mn})\right.$ within $15 \mathrm{~s}$. $\mathrm{CoCl}_{2} \bullet 6 \mathrm{H}_{2} \mathrm{O}$ (Acros Organics) and $\mathrm{NiCl}_{2} \bullet 6 \mathrm{H}_{2} \mathrm{O}$ (Alfa Aesar) were used to prepare Co- $\mathrm{MnO}_{2}$ and $\mathrm{Ni}-\mathrm{MnO}_{2}$ nanosheets at same molar ratio of 0.05 . The resulting dark brown solution was stirred vigorously overnight in the ambient atmosphere at room temperature. The obtained solution was centrifuged using a Sigma 1-14 centrifuge at $1000 \mathrm{~g}$ for $20 \mathrm{~min}$ followed by collecting upper $2 / 3$ of the volume. The collected solution was centrifuged at $15000 \mathrm{~g}$ for $1 \mathrm{~h}$ and the precipitate was washed by water for several times followed by freeze drying. This same synthesis method is used for the preparation of $\mathrm{Co}$ and Ni-doped $\mathrm{MnO}_{2}$ nanosheets.

\subsubsection{Ink preparation}

To prepare printable $\mathrm{Fe}, \mathrm{Co}$ and $\mathrm{Ni}$-doped $\mathrm{MnO}_{2}$ nanosheets ink, the collected precipitate was re-dispersed in a printable solvent consisting of 1:10 propylene glycol (Sigma-Aldrich) : water by mass, $0.06 \mathrm{mg} \mathrm{mL}^{-1}$ Triton X-100 (Sigma-Aldrich). To estimate the concentration of resultant printable inks, the optical absorbance was measured by PerkinElmer UV/VIS/NIR spectrometer Lambda 950S from 800-200 nm wavelength. Concentrations were extracted using Lambert-Beer law $A / l=\alpha_{374} C$, where $A$ is the absorbance, $l=1 \mathrm{~cm}$ is the cell length, $C$ is the concentration of resultant inks and the absorption coefficient $\alpha_{374}=$ $1.13 \times 10^{4} \mathrm{~mol}^{-1} \mathrm{dm}^{3} \mathrm{~cm}^{-1}$. All inks were diluted to the same concentration for inkjet printing. To prepare printable poly (3,4-ethylenedioxythiophene): poly(styrenesulfonate) (PEDOT: PSS) (3.0-4.0\%, Sigma Aldrich) ink, 2 vol\% Triton X-100 and 6 vol\% ethylene glycol (Merck) were added into PEDOT: PSS solution.

\subsubsection{Inkjet printing}

A Dimatix DMP-2800 inkjet printer (Fujifilm Dimatix) equipped with a 16-nozzle cartridge and typical droplet volume of $10 \mathrm{pL}$ was used to print all patterns. To fabricate electrodes for 
three-electrode measurements, $\mathrm{Fe}, \mathrm{Co}, \mathrm{Ni}$-doped and pristine $\delta-\mathrm{MnO}_{2}$ nanosheets inks were printed at $50{ }^{\circ} \mathrm{C}$ with $20 \mu \mathrm{m}$ drop spacing on polyimide substrate in 5 layers, followed by drying at $50{ }^{\circ} \mathrm{C}$ for $2 \mathrm{~h}$. Then, 2 layers of PEDOT: PSS film were printed at room temperature with $20 \mu \mathrm{m}$ drop spacing on top of all $\mathrm{MnO}_{2}$ samples followed by annealing at $120{ }^{\circ} \mathrm{C}$ for 15 $\min$.

\subsubsection{Fabrication of micro-supercapacitors}

All inks were inkjet printed in 5 layers at $20 \mu \mathrm{m}$ drop spacing on a $120 \mu \mathrm{m}$ thick flexible polyimide substrates at $50{ }^{\circ} \mathrm{C}$. Then, 2 layers of PEDOT: PSS were printed at $20 \mu \mathrm{m}$ drop spacing on top of the all $\mathrm{MnO}_{2}$ nanosheets thin films, followed by thermal annealing at $120{ }^{\circ} \mathrm{C}$ for $15 \mathrm{~min}$. The prepared PEDOT: PSS/nanosheets films were used as electrodes for symmetrical MSCs. The PVA/LiCl gel electrolyte was prepared by mixing $1 \mathrm{~g}$ PVA $\left(M_{\mathrm{W}}\right.$ 85000-124000, Aldrich), $2.13 \mathrm{~g} \mathrm{LiCl}$ (Alfa Aesar) and $10 \mathrm{~mL} \mathrm{DI}$ water thoroughly at $85^{\circ} \mathrm{C}$ under vigorous stirring. To complete the fabrication of MSC, the gel electrolyte was deposited on the electrodes area of MSC, and was dried overnight at room temperature.

\subsubsection{Electrochemical characterization}

All electrochemical characterizations were done on an Autolab workstation (PGSTAT128N). The printed thin film electrodes were measured in $0.5 \mathrm{M} \mathrm{Na}_{2} \mathrm{SO}_{4}(\mathrm{ABCR} \mathrm{GmbH})$ and $5 \mathrm{M}$ $\mathrm{LiCl}$ solution in a three-electrode configuration with $\mathrm{Ag} / \mathrm{AgCl}(3 \mathrm{M} \mathrm{KCl})$ electrode (Metrohm) and platinum wire as the reference and counter electrodes, respectively. The allsolid-state MSC was characterized in a two-electrode configuration. Cyclic voltammetry was performed at a scan rate of 5 to $50 \mathrm{mV} \mathrm{s}^{-1}$, galvanostatic charge-discharge curves were measured at current densities from 10 to $100 \mu \mathrm{A} \mathrm{cm} \mathrm{cm}^{-2}$. Electrochemical impedance spectroscopy was performed by applying an AC voltage of $10 \mathrm{mV}$ amplitude in the frequency range from $0.1 \mathrm{~Hz}$ to $10 \mathrm{kHz}$. The high frequency impedance characteristics were interpreted in terms of a $R_{1}\left(R_{2} Q\right)$ equivalent circuit, where $R_{1}$ represents the series resistance of the nanosheet based electrode, $\mathrm{R}_{2}$ its charge transfer resistance, and $\mathrm{Q}$ a constant phase element.

\subsubsection{First principles calculations}

The first principle calculations were carried out within the framework of density functional theory (DFT) using the projector augmented wave (PAW) method ${ }^{30}$ and a plane-wave basis set with a cut-off energy of $500 \mathrm{eV}$ as implemented in the VASP code. ${ }^{31,32}$ In this paper, exchange and correlation effects were described in the local spin density approximation (LSDA) as parameterized by Perdew and Zunger ${ }^{33}$ with an on-site Coulomb interaction U added onto $d$ orbitals. ${ }^{34}$ The effective Coulomb term $U_{\text {eff }}$ of $3.2 \mathrm{eV}, 3.6 \mathrm{eV} 3.1 \mathrm{eV}$, and 2.8 $\mathrm{eV}$ were chosen for $\mathrm{Mn}, \mathrm{Fe}, \mathrm{Co}$, and $\mathrm{Ni} 3 d$ orbitals, respectively. ${ }^{35}$ Monolayers of $\mathrm{MnO}_{2}$ periodically repeated in the $c$ direction were separated by more than $20 \AA$ of vacuum to minimize the interaction. 5 at $\%$ substitutional impurities were modeled using $4 \times 5$ in-plane 
supercells. The atomic positions were relaxed using a $5 \times 4 \times 1 \Gamma$-centered k-point mesh until the forces on each ion were smaller than $0.01 \mathrm{eV} \AA^{-1}$. Spin-polarized calculations were performed with a denser mesh corresponding to $8 \times 8 \times 1 \mathrm{k}$-points.

\subsubsection{Characterization}

Powder X-ray diffraction (XRD) was performed on a Bruker diffractometer (D8 Advance) with $\mathrm{Cu} \mathrm{K} \alpha$ radiation $(\lambda=0.15405 \mathrm{~nm})$. Thin film XRD was measured by a PANalytical X'Pert Pro with $\mathrm{Cu} \mathrm{K} \alpha$ radiation $(\lambda=0.15405 \mathrm{~nm}$ ). AFM (Veeco Dimension Icon) was conducted in standard tapping mode. The AFM data were analyzed by Gwyddion (version 2.47) software. Image-corrected TEM was performed by model Titan 80-300 ST (300 kV) with energy dispersive X-ray spectroscopy (EDS) capability. X-ray photoelectron spectroscopy (XPS) was conducted by an Omicron Nanotechnology GmbH (Oxford Instruments) surface analysis system with a photon energy of $1486.7 \mathrm{eV}$ (Al Ka X-ray source) with a scanning step size of $0.1 \mathrm{eV}$. The pass energy was set to $20 \mathrm{eV}$. The spectra were corrected using the binding energy of $\mathrm{C} 1 s$ of the carbon residual on nanosheets as a reference. High resolution scanning electron microscopy and EDS analyze (HRSEM; Zeiss MERLIN) were performed to acquire information of printed Fe, Co and Ni-doped $\mathrm{MnO}_{2}$ films. Fe-, Co- and Ni-doped $\mathrm{MnO}_{2}$ Raman spectra were recorded on a micro-Raman spectrometer (LabRAM ARAMIS, Horiba-Jobin Yvon, Germany) using a $633 \mathrm{~nm}$ laser. Undoped $\mathrm{MnO}_{2}$ Raman spectra was recorded on a laser-scanning confocal Raman microspectrometer by Krypton laser (Innova 90-K; Coherent, Santa Clara, CA, $\lambda_{\text {exc }} 647.1$ $\mathrm{nm})$.

The specific areal capacitance $\left(C_{\mathrm{A}}\right)$ of film electrodes was calculated from the GCD curves by using Equation (1):

$C_{\mathrm{V}}=[I /(\mathrm{d} V / \mathrm{d} t)] / A_{\text {electrode }}$

where $I$ is the discharge current, $\mathrm{d} V / \mathrm{d} t$ is the slope of discharge curve, and $A_{\text {electrode }}$ refers to the area of the film electrode.

The specific areal capacitance $\left(C_{\mathrm{A} \text {,device }}\right)$ and volumetric capacitance $\left(C_{\mathrm{V} \text {,device }}\right)$ of the MSC devices were also calculated from the GCD curves according to equations (2) and (3), respectively

$$
\begin{aligned}
& C_{\mathrm{A}, \text { device }}=[I /(\mathrm{d} V / \mathrm{d} t)] / A_{\text {device }} \\
& C_{\mathrm{V}, \text { device }}=[I /(\mathrm{d} V / \mathrm{d} t)] / V_{\text {device }}
\end{aligned}
$$

Here $A_{\text {device }}$ refers to the total area of the device including the electrodes and the gap between the electrodes. $V_{\text {device }}$ refers to the total volume of the device, including the volume of the electrodes and the gap between the electrodes. 
The volumetric energy densities $\left(E_{\mathrm{V}}, \mathrm{Wh} \mathrm{cm}^{-3}\right)$ and power densities $\left(P_{\mathrm{V}}, \mathrm{W} \mathrm{cm}{ }^{-3}\right)$ were calculated from equations (4) and (5)

$E_{\mathrm{V}}=C_{\mathrm{V}, \text { device }} V^{2} /(2 \times 3600)$

$P_{\mathrm{V}}=3600 \times E_{\mathrm{V}} / \Delta t$

Where $\Delta t$ refers to discharge time.

\subsection{Results and discussion}

\subsubsection{Nanosheets characterizations}
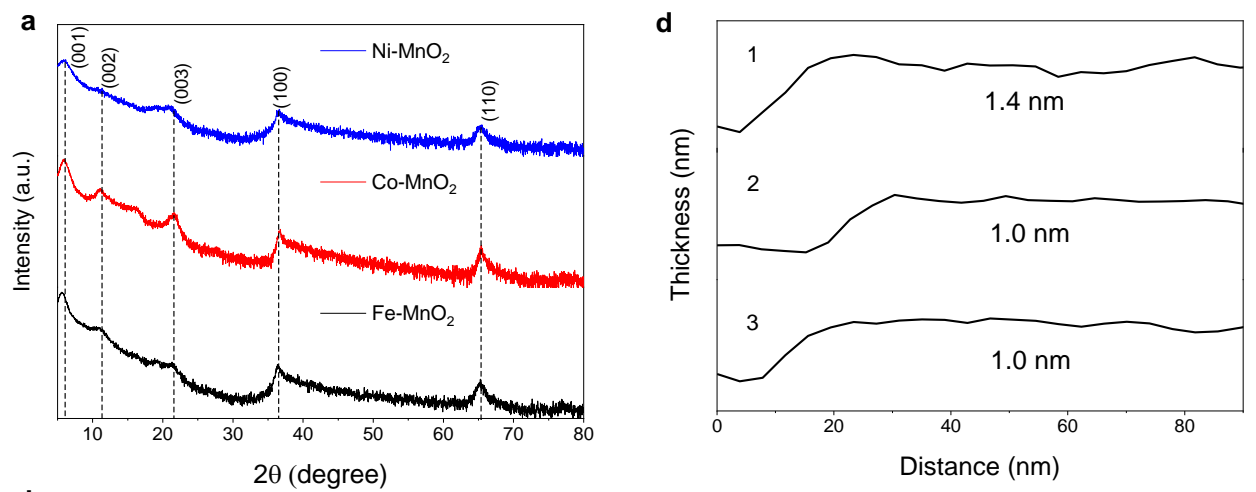

b

C
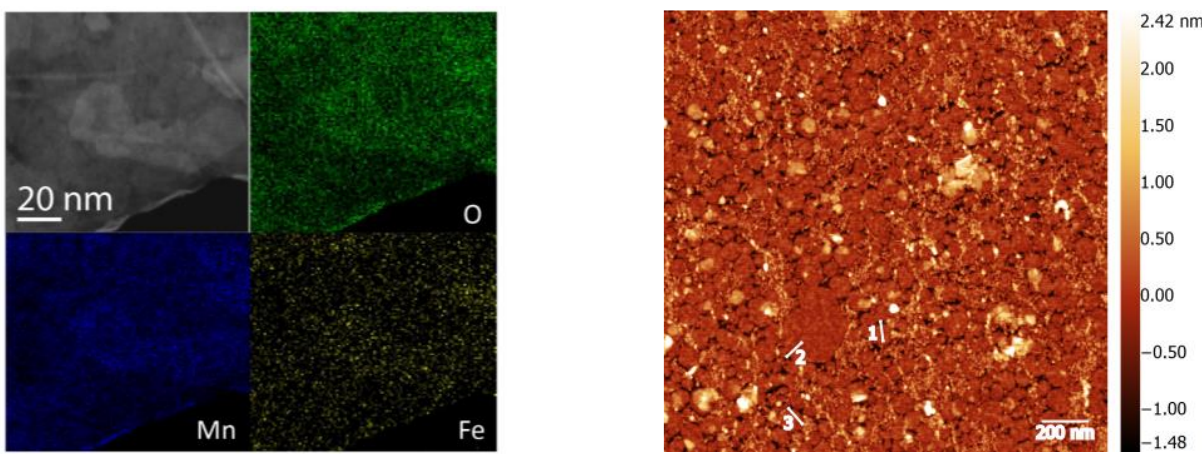

Figure 3.1 Characterization of nanosheets. (a) XRD of Fe, Co and Ni-doped $\mathrm{MnO}_{2}$ nanosheets. (b) TEM and EDS elemental mapping of $\mathrm{Fe}-\mathrm{MnO}_{2}$ nanosheets. (c) AFM images of $\mathrm{Fe}-\mathrm{MnO}_{2}$ nanosheets. (d) The corresponding height profiles of nanosheets (the numbers of 1,2 and 3 correspond to the line scan number in Figure 3.1c). 
a

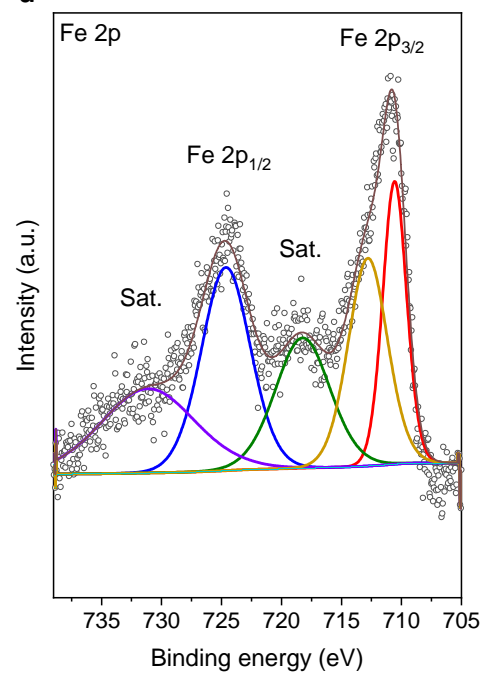

C

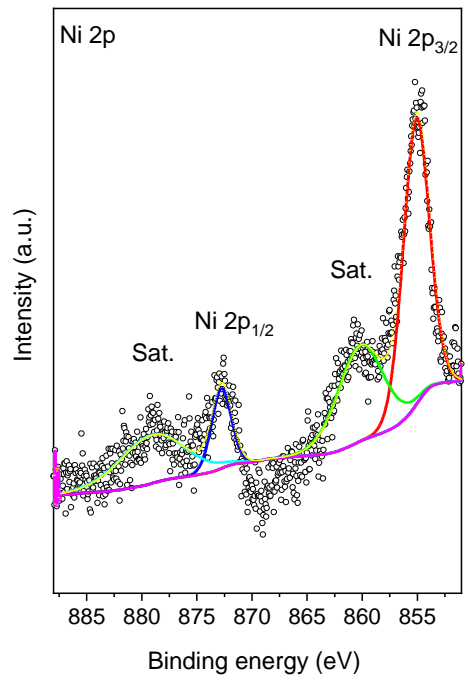

b

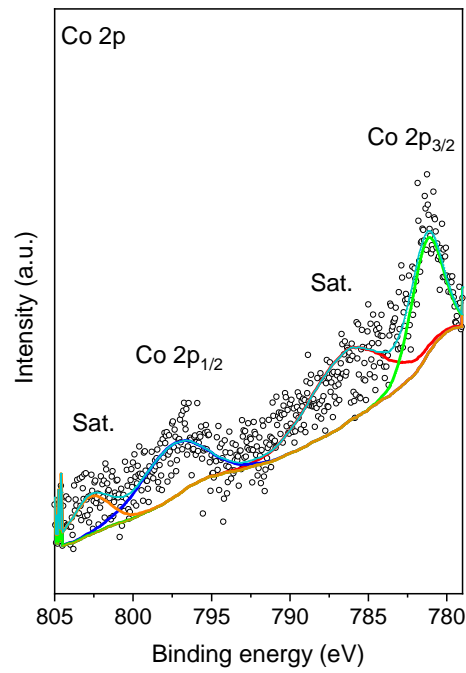

d

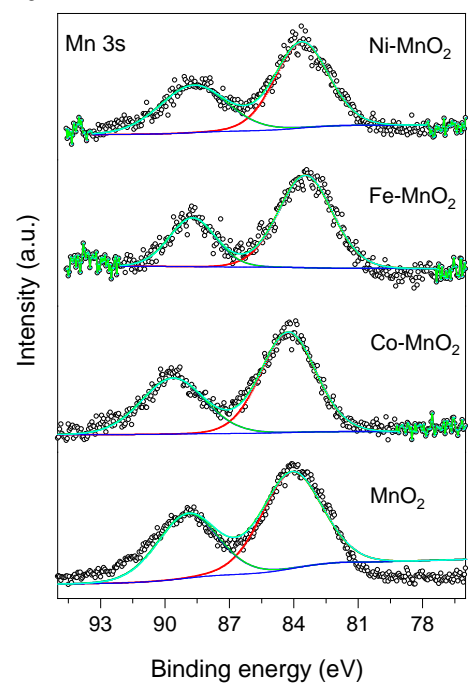

Figure 3.2 High resolution XPS spectra of (a) $\mathrm{Fe} 2 p$, (b) Co $2 p$ and (c) $\mathrm{Ni} 2 p$ of $\mathrm{Fe}$, Co and Ni-doped $\mathrm{MnO}_{2}$ nanosheets, respectively. (d) High resolution XPS spectra of $\mathrm{Mn} 3 s$ of pristine and $\mathrm{Fe}$, Co and $\mathrm{Ni}$-doped $\mathrm{MnO}_{2}$ nanosheets.

The crystal structures and phase information of the $\mathrm{Fe}$, $\mathrm{Co}$ and $\mathrm{Ni}$-doped $\mathrm{MnO}_{2}$ nanosheets (Referred to as $\mathrm{Fe}-\mathrm{MnO}_{2}, \mathrm{Co}-\mathrm{MnO}_{2}$ and $\mathrm{Ni}-\mathrm{MnO}_{2}$, respectively) were acquired from powder $\mathrm{XRD}$ of freeze-dried samples as shown in Figure 3.1a. All the patterns show the reflections of (001) series that indicate the laminar and ultrathin features of the prepared samples. Two 
asymmetrical in-plane (100) and (110) reflections at $2 \theta=36^{\circ}$ and $65^{\circ}$ are observed in all samples, indicating a hexagonal unit cell and the layered birnessite-type structure in $\mathrm{Fe}, \mathrm{Co}$ and Ni-doped $\mathrm{MnO}_{2}$ nanosheets. ${ }^{36}$ The small differences between the (002) diffraction peak angles can be explained by small variations in the spacings between nanosheets in different restacked 2D structures. Element mappings from energy-dispersive spectroscopy (EDS) show homogeneous spatial distributions of Fe (Figure 3.1b), Co and Ni (Figure S3.1, Appendices) in the basal plane of $\mathrm{MnO}_{2}$ and the incorporation of foreign atoms into the $\mathrm{MnO}_{2}$ nanosheet lattice. The solubility of $\mathrm{Fe}, \mathrm{Co}$ and $\mathrm{Ni}$ in the $\mathrm{MnO}_{2}$ nanosheet crystal structure can be attributed to the similar ionic radius, oxidation state and electronegativity. The thickness of nanosheets, deposited on silicon substrates by the Langmuir-Blodgett (LB) method, ${ }^{37}$ as determined by atomic force microscopy (AFM), showed average thicknesses of $1.0-1.8 \mathrm{~nm}$ for $\mathrm{Fe}-\mathrm{MnO}_{2}$ (Figure 3.1c,d), $\mathrm{Co}-\mathrm{MnO}_{2}$ and $\mathrm{Ni}-\mathrm{MnO}_{2}$ (Figure S3.2, Appendices), respectively, in accordance with the reported AFM thicknesses of $\mathrm{MnO}_{2}$ nanosheets in our previous report. ${ }^{38}$ The variation in thickness and difference with the crystallographic thickness, which is $0.52 \mathrm{~nm}$ based on its atomic architecture, is attributed to hydration and the presence of organic ions, i.e., $\mathrm{TBA}^{+}$on both sides of the nanosheets. ${ }^{36,39}$ The lateral sizes of $\mathrm{Fe}, \mathrm{Co}$ and $\mathrm{Ni}$-doped $\mathrm{MnO}_{2}$ nanosheets estimated from AFM images indicate that the majority of nanosheets have lateral sizes between $50-150 \mathrm{~nm}, 40-120 \mathrm{~nm}$ and 40-140 nm for $\mathrm{Fe}$, Co and Ni-doped $\mathrm{MnO}_{2}$ nanosheets, respectively (Figure S3.3, Appendices), which are suitable dimensions for ink jetting. ${ }^{10}$

X-ray photoelectron spectroscopy (XPS) scans in Figure S3.4a-c (Appendices) reveal the presence of $\mathrm{Fe}$, $\mathrm{Co}$ and $\mathrm{Ni}$ elements in $\mathrm{Fe}, \mathrm{Co}$ and $\mathrm{Ni}$-doped $\mathrm{MnO}_{2}$, respectively, which is consistent with the EDS mapping data. The high resolution spectrum of Fe $2 p$ shows two binding energy (BE) peaks that can be assigned to $\mathrm{Fe} 2 p_{3 / 2}$ and $\mathrm{Fe} 2 p_{1 / 2}$, respectively (Figure 3.2a). The BE difference of $13.7 \mathrm{eV}$ indicates the presence of $\mathrm{Fe}^{3+} .{ }^{40}$ The high resolution spectrum of Co $2 p$ shows two BE peaks at 781.3 and $797.5 \mathrm{eV}$ that can be assigned to Co $2 p_{3 / 2}$ and Co $2 p_{1 / 2}$, respectively (Figure 3.2b), indicating the existence of $\mathrm{Co}^{2+} .{ }^{41}$ The high resolution Ni $2 p$ spectrum in Figure $3.2 \mathrm{c}$ shows two BE peaks that are Ni $2 p_{3 / 2}$ at 855.1 and $\mathrm{Ni} 2 p_{1 / 2}$ at $872.8 \mathrm{eV}$, and two broad satellites peaks which can be assigned to $\mathrm{Ni}^{2+} .42$ The high resolution XPS of Mn $2 p$ shows spin-orbit doublet corresponding to the Mn $2 p_{1 / 2}$ and Mn $2 p_{3 / 2}$ states (Figure S3.4d, Appendices) which can be used to roughly determine the oxidation state of Mn. However, due to the complex oxidation and spin states in the Mn $2 p$ spectrum, the splitting of Mn $3 s$ peaks is normally used as a probe to determine the oxidation state of $\mathrm{Mn}$, as shown in Figure 3.2d. The $\Delta E$ values are $4.8 \mathrm{eV}$ for pristine $\delta-\mathrm{MnO}_{2}$ nanosheets, 5.2 $\mathrm{eV}$ for $\mathrm{Fe}-\mathrm{MnO}_{2}$ nanosheets, $5.3 \mathrm{eV}$ for $\mathrm{Co}-\mathrm{MnO}_{2}$ nanosheets and $5.0 \mathrm{eV}$ for Ni-MnO nanosheets, indicating the coexistence of $\mathrm{Mn}^{3+}$ and $\mathrm{Mn}^{4+}$ in $\mathrm{Fe}$, Co and Ni-doped $\mathrm{MnO}_{2}$ nansoheets. In order to maintain charge neutrality, it is likely that oxygen vacancies were also present in the structure, and these have been shown to occur in $\mathrm{MnO}_{2}$ nanosheets. ${ }^{43}$ Furthermore, the Raman spectra in Figure S3.5 (Appendices) confirm that undoped and 
doped $\mathrm{MnO}_{2}$ nanosheets have their characteristic peaks at similar positions, indicating that the structure of $\mathrm{MnO}_{2}$ was maintained upon doping.

\subsubsection{Formulation of water-based printable nanosheets inks}

a

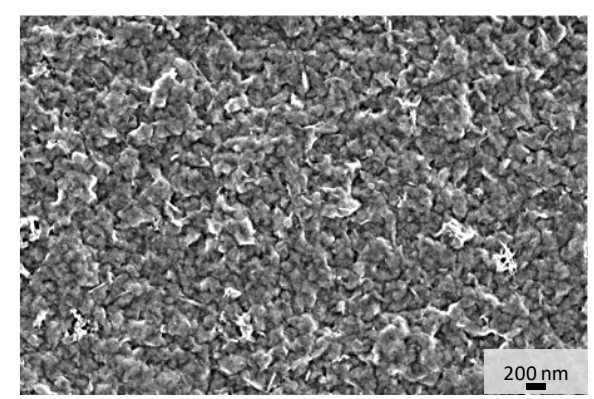

b

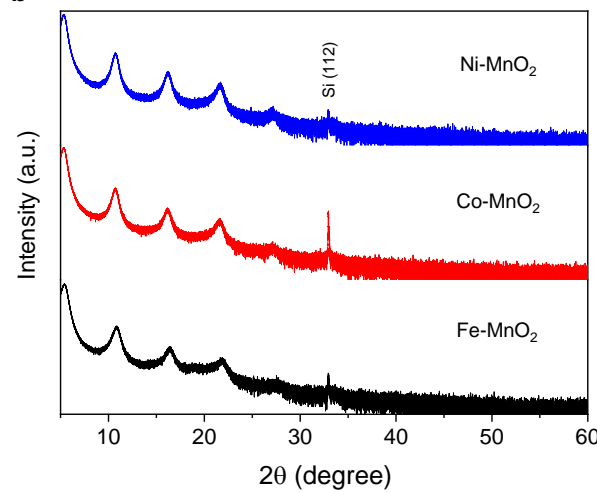

Figure 3.3 (a) Top-view SEM images of printed $\mathrm{Fe}-\mathrm{MnO}_{2}$ nanosheets film on silicon substrates. (b) XRD patterns of printed $\mathrm{Fe}, \mathrm{Co}$ and $\mathrm{Ni}$-doped $\mathrm{MnO}_{2}$ nanosheets films.

Water-based inks were developed following the protocol reported in our previous work. ${ }^{38}$ The ink formulation was optimized to have optimal rheological properties for inkjet printing. The surface tension and viscosity of water-based inks were $46 \mathrm{mN} \mathrm{m}^{-1}$ and $1.7 \mathrm{mPa} \mathrm{s}$, respectively. ${ }^{38}$ As an example, the good quality viscoelastic properties and stability of the $\mathrm{Fe}-\mathrm{MnO}_{2}$ ink is illustrated by the stroboscopic images of ink droplet formation versus time; no satellite droplets can be seen (Figure S3.5, Appendices). Top-view SEM images of inkjetprinted Fe (Figure 3.3a), Co and Ni-doped $\mathrm{MnO}_{2}$ nanosheets (Figure S3.6, Appendices) films on silicon substrates indicate uniform deposition of nanosheets and good film continuity across a large surface area. The surface roughness of printed films by AFM at different length scales (Figure S3.7, Appendices) showed similar root mean square (RMS) values. The scan area does not have a large influence on the RMS roughness values, which indicates highly uniform printed nanosheet films. The XRD of all films in Figure 3.3b show (001) series reflections at $2 \theta 5.3^{\circ}, 10.7^{\circ}, 15.9^{\circ}, 21.6^{\circ}$ and $27.1^{\circ}$, originating from the (001), (002), (003), (004) and (005) reflections, respectively. The presence of higher order basal peaks indicates highly $c$ oriented and laminar features with a layer spacing of $1.6 \mathrm{~nm}$. This distance is large enough to facilitate ion diffusion between the interdigitated electrodes of MSCs. The doping concentrations were estimated by EDS and were found to be about 5.3\%, 5.3\% and $4.7 \%$ for $\mathrm{Fe}$, $\mathrm{Co}$ and $\mathrm{Ni}$-doped $\mathrm{MnO}_{2}$ nanosheets, respectively (Figure S3.8, Appendices), in accordance with the nominal element compositions targeted during synthesis. 


\subsubsection{Three-electrode measurement of printed electrodes}

Thin film electrodes fabricated by printing PEDOT: PSS/ $\mathrm{MnO}_{2}$ nanosheet films were studied in a three-electrode electrochemical setup. The cyclic voltammetry $(\mathrm{CV})$ curves of $\mathrm{Fe}, \mathrm{Co}$ and Ni-doped $\mathrm{MnO}_{2}$ electrodes at a scan rate of $5 \mathrm{mV} \mathrm{s}^{-1}$ in Figure 3.4a show broad redox peaks that contribute more capacitance than the corresponding undoped $\delta-\mathrm{MnO}_{2}$ electrode in the potential window from 0 to $1 \mathrm{~V}$ (versus $\mathrm{Ag} / \mathrm{AgCl}$ ), as demonstrated by the area integrated within the current-potential curves. The electrochemical activity of the $\mathrm{Fe}-\mathrm{MnO}_{2}$ electrode is clearly much larger than that of the $\mathrm{Co}-\mathrm{MnO}_{2}, \mathrm{Ni}-\mathrm{MnO}_{2}$ and pristine $\delta-\mathrm{MnO}_{2}$ electrodes. Notably, the pair of redox peaks of $\mathrm{Fe}-\mathrm{MnO}_{2}$ at 0.72 and $0.46 \mathrm{~V}$ provide more redox-active sites than pure $\mathrm{MnO}_{2}$, arising from the pseudocapacitance generated by the faradic redox reactions of mixed valent $\mathrm{MnO}_{2}$ and doped $\mathrm{Fe}^{3+}$, which was also confirmed in a $5 \mathrm{M} \mathrm{LiCl}$ electrolyte (Figure S3.9, Appendices). ${ }^{44}$ The governing surface redox reactions are as follows:

$$
\begin{aligned}
& \mathrm{MnO}_{2}+\mathrm{Na}^{+}+\mathrm{e}^{-} \rightleftarrows \mathrm{MnOONa} . \\
& \mathrm{Fe}(\mathrm{III})+\mathrm{e}^{-} \rightleftarrows \mathrm{Fe}(\mathrm{II})
\end{aligned}
$$

a

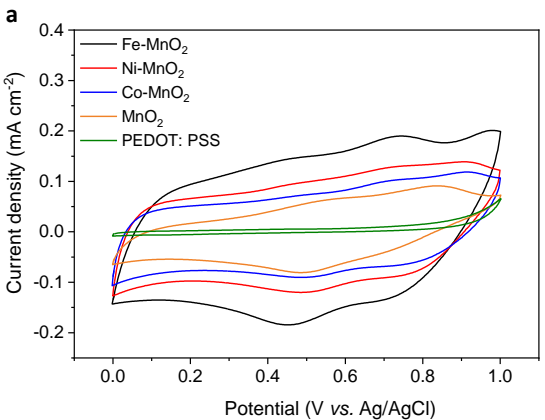

C

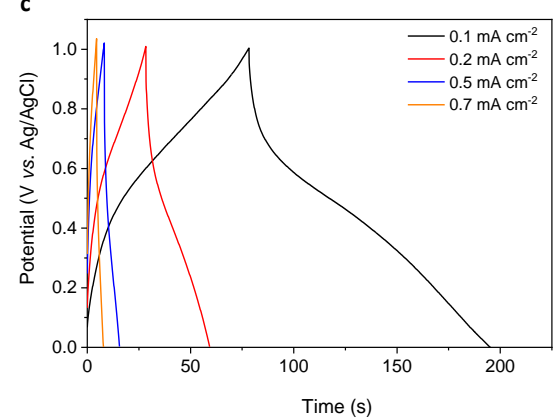

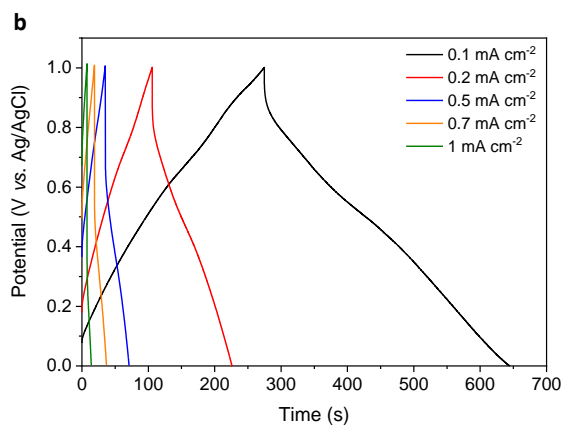

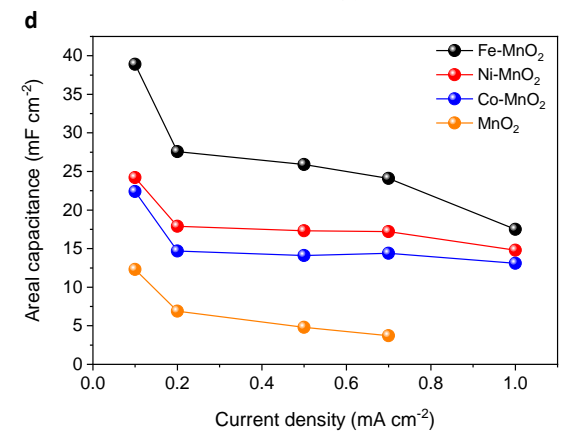

Figure 3.4 Three-electrode characterizations in $0.5 \mathrm{M} \mathrm{Na}_{2} \mathrm{SO}_{4}$ electrolyte. (a) CV curves of Fe, Co, $\mathrm{Ni}$-doped $\mathrm{MnO}_{2}$, pristine $\delta-\mathrm{MnO}_{2}$ and PEDOT: PSS electrodes at $5 \mathrm{mV} \mathrm{s}^{-1}$. GCD of (b) Fe, (c) undoped $\delta-\mathrm{MnO}_{2}$ electrodes at different current densities. (d) Areal capacitance $\mathrm{C}_{\mathrm{A}}$ of $\mathrm{Fe}, \mathrm{Co}, \mathrm{Ni}$-doped and pristine $\delta-\mathrm{MnO}_{2}$ electrodes at different current densities. 
The higher specific capacitance of the Fe-doped $\mathrm{MnO}_{2}$ electrode compared with Co and Nidoped $\mathrm{MnO}_{2}$ is confirmed by the galvanostatic charge/discharge (GCD) curves between 0 to $1 \mathrm{~V}$ at varying current densities (Figure 3.4b-c, Figure S3.10, Appendices). The potential does not show a linear change with time, a indicating faradaic redox reaction during charging and discharging. The bare PEDOT: PSS film showed a smaller current response than the electrodes, indicating the negligible contribution of PEDOT: PSS to the overall capacitance. The areal capacitances of all samples are shown in Figure 3.4d. At current density of $0.1 \mathrm{~mA}$ $\mathrm{cm}^{-2}$, the areal capacitance is $39 \mathrm{mF} \mathrm{cm}{ }^{-2}$ for $\mathrm{Fe}-\mathrm{MnO}_{2}$ electrode, $22 \mathrm{mF} \mathrm{cm}{ }^{-2}$ for Co- $\mathrm{MnO}_{2}$ electrode, $24 \mathrm{mF} \mathrm{cm}{ }^{-2}$ for $\mathrm{Ni}-\mathrm{MnO}_{2}$ electrode and $12 \mathrm{mF} \mathrm{cm}{ }^{-2}$ for the pristine $\delta-\mathrm{MnO}_{2}$ electrode. Electrochemical impedance spectroscopy (EIS) was employed to study the series resistance and charge transfer resistance of all electrodes, as shown in Figure S3.11 (Appendices). All doped $\mathrm{MnO}_{2}$ electrodes exhibited lower series resistance (indicated by the real axis value at high frequency intercept) and lower charge transfer resistance (indicated by the radii of the semicircle in the high frequency region) than undoped $\mathrm{MnO}_{2}$ electrode. Specifically, $\mathrm{Fe}-\mathrm{MnO}_{2}$ electrode showed the lowest series resistance and charge transfer resistance of all samples (Table S1, Appendices). These experimental data clearly demonstrate that substitutional doping improves both the electronic conductivity of the $\mathrm{MnO}_{2}$ nanosheets, as well as their electron transfer kinetics.

\subsubsection{First principles calculations}
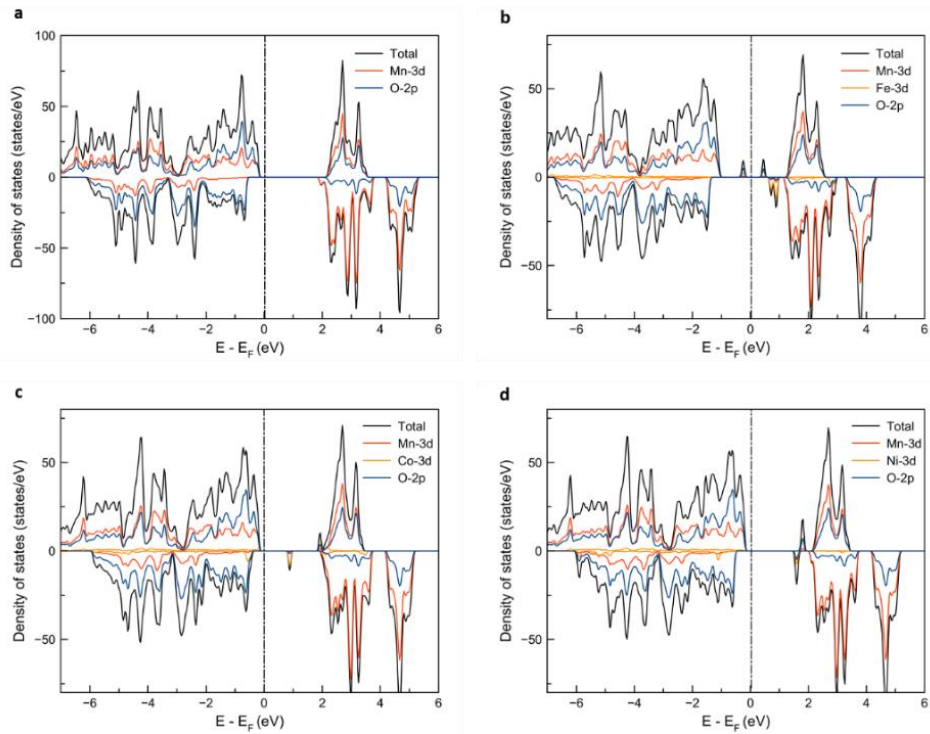

Figure 3.5 First principles calculations. DOS of (a) pristine, (b) $5 \% \mathrm{Fe}$, (c) $5 \%$ Co and (d) $5 \% \mathrm{Ni}$-doped $\mathrm{MnO}_{2}$ nanosheets. 
First principles calculations were carried out to gain further insight into the effect of aliovalent doping on the electronic properties of $\mathrm{MnO}_{2}$ nanosheets. The band structures are shown in Figure S3.12 (Appendices). The model structure used here, a 2D 4x5 $\mathrm{MnO}_{2}$ supercell with one dopant atom at its center, may be simplified with respect to the real defect structure of doped $\mathrm{MnO}_{2}$, but it does provide qualitative insight into the general trends of foreign element doping on the band structure of (doped) $\mathrm{MnO}_{2}$. The partial density of state (PDOS) of pristine $\delta-\mathrm{MnO}_{2}$ nanosheets shown in Figure 3.5a indicates semiconducting behavior with a band gap of $2.16 \mathrm{eV}$, which is in good agreement with the reported experimental band gap of $2.23 \mathrm{eV} .{ }^{45}$ By introducing 5\% Fe doping, occupied and unoccupied impurity states emerge in the fundamental band gap so that the chemical potential is increased, which effectively decreases the band gap and increases the mobile charge carrier density (Figure 3.5b). Only empty impurity states form in the band gap for Ni and Co doped systems (Figure 3.5c-d) and these do not contribute much to the carrier density as illustrated in Figure 3.4. The origin of the difference lies in the competition of crystal field splitting and spin polarization of dopants. In an $\mathrm{O}_{6}$ octahedral crystal field, the $3 d$ orbitals split into low lying $t_{2 \mathrm{~g}}\left(d_{\mathrm{xz}}, d_{\mathrm{yz}}, d_{\mathrm{xy}}\right)$ and high lying $e_{\mathrm{g}}\left(d_{\mathrm{x}}{ }^{2} \mathrm{y}^{2}, d_{\mathrm{z}}{ }^{2}\right)$ states which can give rise to high spin states (HS) and low spin states (LS)) depending on the competition between the crystal field and exchange interactions. Because of the large exchange splitting of Fe compared to that of Co and $\mathrm{Ni},{ }^{46}$ the electrons in $\mathrm{Fe}^{4+}\left(d^{4} s^{0}\right)$ would be in a HS state occupying the higher $e_{\mathrm{g}}$ levels close to the conduction band, while in $\mathrm{Co}^{4+}\left(d^{5} s^{0}\right)$ and $\mathrm{Ni}^{4+}\left(d^{6} s^{0}\right)$ the electrons are in LS states occupying $t_{2 \mathrm{~g}}$ states and mixing with valence band which contribute less to the carrier density. The partial charge density of impurity states near chemical potential for $\mathrm{Fe}-\mathrm{MnO}_{2}$ (Figure S3.13a-b, Appendices) show clear $e_{\mathrm{g}}$ orbital character $\left(d_{\mathrm{x}-\mathrm{y}}^{2}{ }^{2}, d_{\mathrm{z}}{ }^{2}\right)$. Therefore, the resulting improved electronic conductivity of $\mathrm{Fe}-\mathrm{MnO}_{2}$ is likely to contribute directly to the improved electrochemical behavior as compared with pristine $\delta-\mathrm{MnO}_{2}$ nanosheets. In contrast, as shown in Figure 3.5c-d, the presence of Co or Ni atoms in the $\mathrm{MnO}_{2}$ lattice does not reduce the band gap of $\mathrm{MnO}_{2}$ (Figure S3.12c-d, Appendices). The partial charge density distribution shows weak hybridization of O $2 p$ orbitals with Co $3 d$ orbitals (Figure S3.13c, Appendices) and strong hybridization of $\mathrm{O} 2 p$ orbitals with Ni $3 d$ orbitals (Figure S3.13d, Appendices); the charges around $\mathrm{Ni}$ atoms in $\mathrm{Ni}-\mathrm{MnO}_{2}$ are more delocalized than those around Co atoms in $\mathrm{Co}-\mathrm{MnO}_{2}$. However, the change in band gap is much smaller than that for Fe- $\mathrm{MnO}_{2}$. So the $\mathrm{Fe}-\mathrm{MnO}_{2}$ exhibits a significant increase in the carrier density and conductivity, while doping with $\mathrm{Ni}$ or Co has a much more limited effect on conductivity (Figure 3.4). The Tauc plot further confirm the effect of doping on the band gap of $\mathrm{MnO}_{2}$ nanosheets (Figure S3.14, Appendices). The band gap of Fe-doped $\mathrm{MnO}_{2}(1.86 \mathrm{eV})$ is smaller than that of Co-doped $(1.92 \mathrm{eV})$ and $\mathrm{Ni}$-doped $(1.90 \mathrm{eV}) \mathrm{MnO}_{2}$. The band gaps of all doped $\mathrm{MnO}_{2}$ compositions is smaller than that of undoped $\mathrm{MnO}_{2}(2.14 \mathrm{eV})$.

\subsubsection{Electrochemical performance of printed MSCs}


a

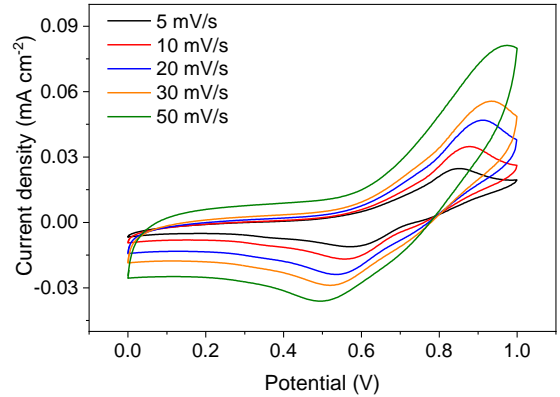

C

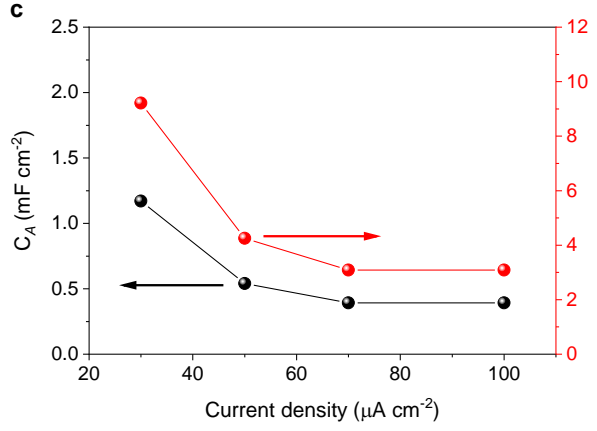

b

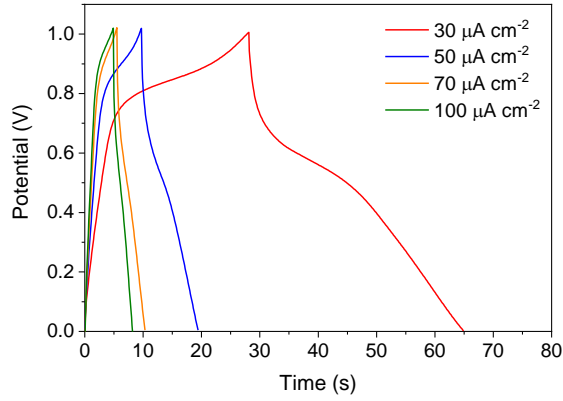

d

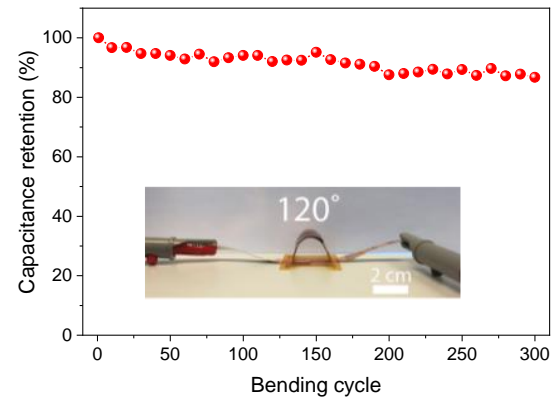

Figure 3.6 Printed $\mathrm{Fe}-\mathrm{MnO}_{2}$ based MSC electrochemical characterizations in PVA/LiCl electrolyte. (a) CV of MSC at scan rates from 5 to $50 \mathrm{mV} \mathrm{s}^{-1}$. (b) Galvanostatic charge-discharge of MSC at current densities from 10 to $100 \mu \mathrm{A}$ $\mathrm{cm}^{-2}$. (c) Areal capacitance and volumetric capacitance at different current densities. (d) Bending test of MSC with bending radius of about $2 \mathrm{~cm}$ at a current density of $50 \mu \mathrm{A} \mathrm{cm}^{-2}$. The inset is the optical image of MSC under bending radius of about $2 \mathrm{~cm}$.

A symmetrical MSC with interdigitated $\mathrm{Fe}-\mathrm{MnO}_{2}$ nanosheet-based electrodes was printed on a polyimide substrate, and $\mathrm{CV}$ and galvanostatic charge-discharge measurements were conducted. The $\mathrm{CV}$ curves at scan rates between 5 and $50 \mathrm{mV} \mathrm{s}^{-1}$ (Figure 3.6a) are confirmed in the GCD curves (Figure 3.6b). The CV curves in Figure 3.6a are qualitative match with three-electrode measurements in Figure 3.4a. The possible reasons for the Faradaic character in Figure 3.6a are the lower ions mobility in gel electrolyte compare with liquid electrolyte and polarizations effect. The areal capacitances and volumetric capacitances calculated from the GCD curves (Figure 3.6c) showed that the highest areal and volumetric capacitances are $2.4 \mathrm{mF} \mathrm{cm}^{-2}$ and $18.9 \mathrm{~F} \mathrm{~cm}^{-3}$ at a current density of $10 \mu \mathrm{A} \mathrm{cm}^{-2}$, respectively. Notably, the surface area used for the calculation of the capacitance includes the area and the gap between the electrodes, while the volume in the calculation included the volume of the electrodes and the spatial gap between the electrodes. The MSC showed $86.7 \%$ capacitance retention after 300 bending cycles with bending radius of about $2 \mathrm{~cm}$ (Figure 3.6d), and 78.7\% capacitance retention after 5200 charge-discharge cycles without bending (Figure 3.7a), indicating good cycling stability. 

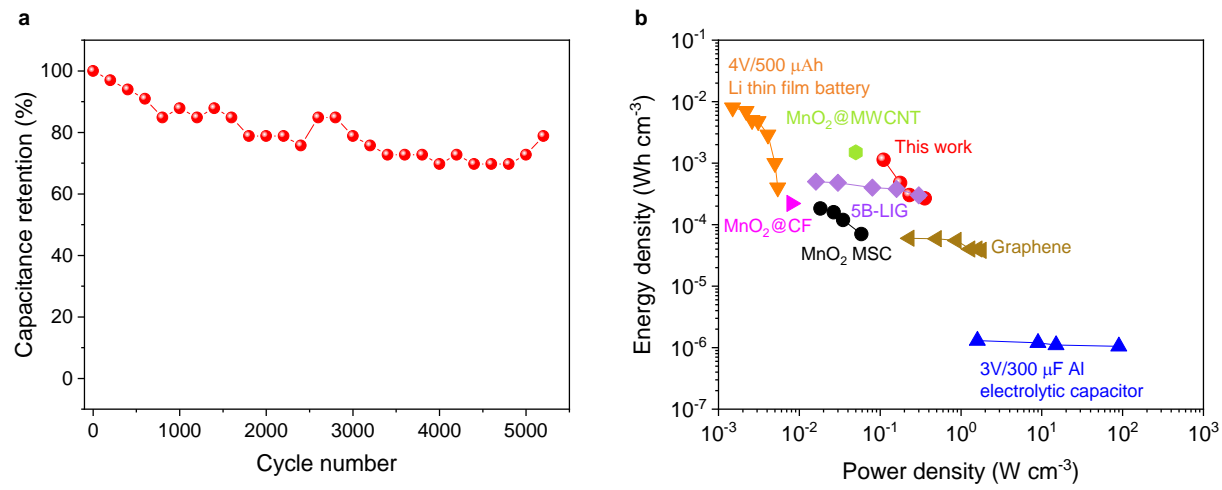

Figure 3.7 (a) Cyclability test of MSC at a current density of $70 \mu \mathrm{A} \mathrm{cm}{ }^{-2}$. (b) Ragone plot of MSC and recent data from literature.

The MSC reached the highest volumetric energy density $\left(E_{\mathrm{V}}\right)$ of $2.5 \times 10^{-3} \mathrm{Wh} \mathrm{cm}^{-3}$ at a volumetric power density $\left(E_{\mathrm{P}}\right)$ of $4.2 \times 10^{-2} \mathrm{~W} \mathrm{~cm}^{-3}$, which is superior to the performance of MSCs based on inkjet-printed $\delta-\mathrm{MnO}_{2}$ nanosheets (Figure 3.7b). ${ }^{38}$ The $E_{\mathrm{V}}$ of MSC is higher than commercial $3 \mathrm{~V} / 300 \mu \mathrm{F}$ Al electrode capacitors, ${ }^{47}$ and the $E_{\mathrm{P}}$ of our MSC is higher than that of a $4 \mathrm{~V} / 500 \mu \mathrm{Ah}$ lithium thin film battery. ${ }^{47}$ Although the $3 \mathrm{~V} / 300 \mu \mathrm{F}$ Al electrode capacitors and $4 \mathrm{~V} / 500 \mu \mathrm{Ah}$ lithium thin film battery show higher power density and energy density than our MSC, respectively, the Fe-doped $\mathrm{MnO}_{2}$ based MSC demonstrated here is performing better when considering the combination of energy and power density. Moreover, the performance of printed $\mathrm{Fe}-\mathrm{MnO}_{2}$ nanosheet MSCs is comparable to or better than that of other devices based on $\mathrm{MnO}_{2} @$ carbon fibers, ${ }^{48}$ graphene, ${ }^{49} \mathrm{MnO}_{2} @$ multiwalled carbon nanotube (MWCNT), ${ }^{50}$ and boron-doped laser-induced graphene (B-LIG). ${ }^{51}$

To test the mechanical flexibility and ductility in conjunction with their electrochemical performance, the $\mathrm{CV}$ characteristic was measured at different bending angles (Figure 3.8a). The CV curves remained unaffected when the devices with the device area of around $1.2 \mathrm{~cm}^{2}$ (Figure 3.8b) and electrodes thickness around $1.3 \mu \mathrm{m}$ were bent with a bending radius of about $2 \mathrm{~cm}$. The cross-sectional SEM image of a printed $\mathrm{Fe}-\mathrm{MnO}_{2}$ film and corresponding optical microscopy images of printed electrodes and the interspace between them are shown in Figure S3.16 (Appendices). The self-discharge rate of MSCs is a major issue for practical applications. During self-discharge, a small amount of leakage current could cause the voltage decay of a charged supercapacitor over time. ${ }^{52}$ As shown in Figure 3.8c, the MSC shows an ultra-small leakage current of $<700 \mathrm{nA}$ after $10 \mathrm{~h}$, compared with $5 \mu \mathrm{A}$ for commercial SCs. ${ }^{52}$ Since most SCs are working in the range of $\mathrm{V}_{\max }$ to $1 / 2 \mathrm{~V}_{\max },{ }^{53}$ the selfdischarge time for such a voltage drop is practically relevant. The Fe- $\mathrm{MnO}_{2} \mathrm{MSC}$ selfdischarges from $1 \mathrm{~V}$ to $0.5 \mathrm{~V}$ in about $12 \mathrm{~h}$ (Figure 3.8d), which is comparable with two commercial supercapacitors that show self-discharge rates of $8 \mathrm{~h}$ and $21 \mathrm{~h},{ }^{52}$ thus suggesting 
that the $\mathrm{Fe}-\mathrm{MnO}_{2} \mathrm{MSC}$ is a promising candidate material for practical applications that require long lifetime and high reliability.

a

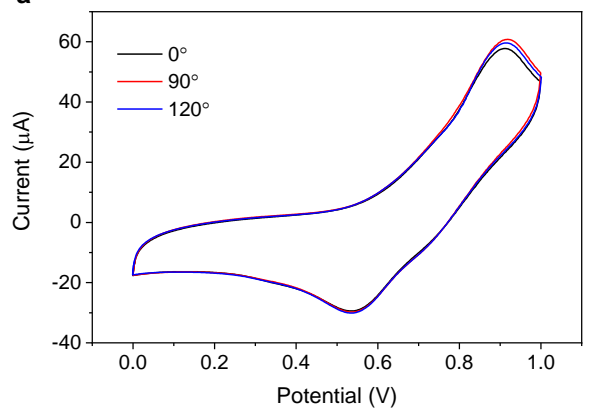

C

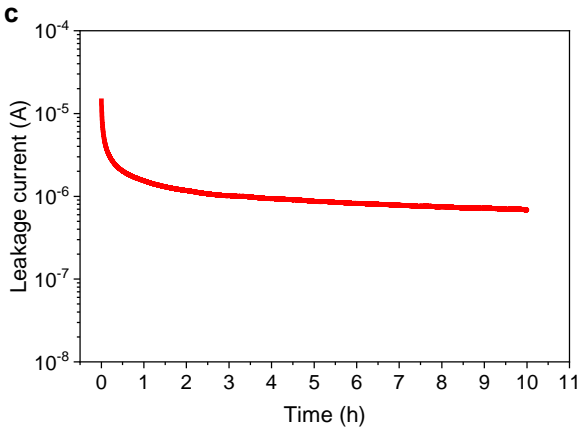

b

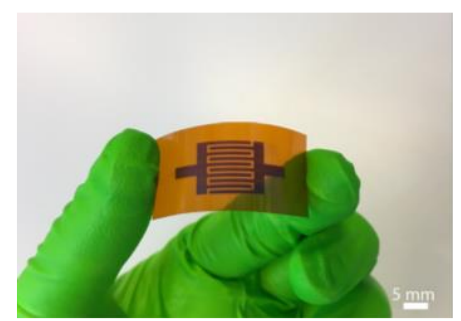

d

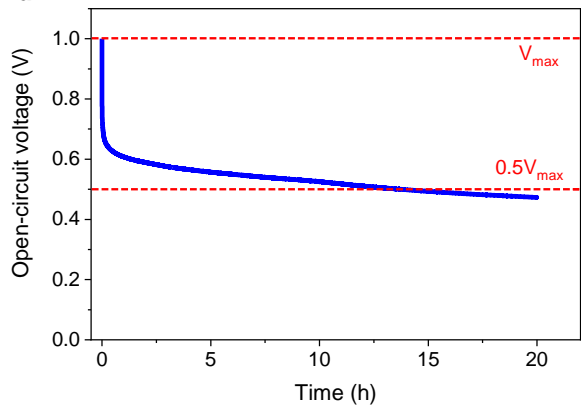

Figure 3.8 Flexibility and discharge measurements. (a) $\mathrm{CV}$ of $\mathrm{Fe}-\mathrm{MnO}_{2} \mathrm{MSC}$ under different bending angle at scan rate of $20 \mathrm{mV} \mathrm{s}^{-1}$. The inset shows optical images of the MSC under different bending angles. (b) Optical image of printed micro-supercapacitor. (c) Leakage current measurement of Fe- $\mathrm{MnO}_{2} \mathrm{MSC}$. A DC voltage of $\mathrm{V}_{\max }=1 \mathrm{~V}$ was applied to the MSC and the current required to retain that voltage was measured for $10 \mathrm{~h}$. (d) Self-discharging of MSC, obtained by charging the MSC to $\mathrm{V}_{\max }$ and then measuring the change of open-circuit voltage from $\mathrm{V}_{\max }$ to $0.5 \mathrm{~V}_{\max }$.

\subsection{Conclusions}

In summary, we have demonstrated atomic-level engineering of $\mathrm{MnO}_{2}$ nanosheets by substitutional doping with $\mathrm{Fe}, \mathrm{Co}$ and $\mathrm{Ni}$ atoms. The theoretical calculations show the excellent electrochemical behavior of Fe-doped $\mathrm{MnO}_{2}$ nanosheets can be attributed to the new electronic states emerging near the Fermi level that increase the electronic conductivity and form additional surface redox sites. Owing to their excellent dispersibility in water, the $\mathrm{Fe}-, \mathrm{Co}-$ and $\mathrm{Ni}-\mathrm{MnO}_{2}$ nanosheets could be formulated directly in water-based stable inks. As best performing candidate among the tested compositions, $\mathrm{Fe}-\mathrm{MnO}_{2}$ nanosheet ink was used to fabricate all-solid-state flexible MSCs on polyimide substrates by inkjet printing 
technology. The printed MSC exhibited superior performance in terms of the combination of high energy density and high power density when compared to other state of the art concepts, and commercially available products on the market. In comparison with undoped $2 \mathrm{D} \mathrm{MnO}_{2}$ MSCs ${ }^{38}$ both the power density and the energy density increased by a factor of more than 6 . The printed $\mathrm{Fe}-\mathrm{MnO}_{2} \mathrm{MSC}$ showed good cycling stability and good mechanical properties in terms of flexibility and ductility. Overall, both inkjet-printed all-solid-state MSCs and the proposed substitutional doping strategy hold great potential to develop next-generation high energy and high power energy storage devices for portable electronics and wearable electronics applications.

\subsection{References}

1. ten Elshof, J. E., Yuan, H. \& Gonzalez Rodriguez, P. Two-dimensional metal oxide and metal hydroxide nanosheets: synthesis, controlled assembly and applications in energy conversion and storage. Adv. Energy Mater. 6, 1600355 (2016).

2. Bonaccorso, F. et al. Graphene, related two-dimensional crystals, and hybrid systems for energy conversion and storage. Science 347, 1246501 (2015).

3. Da, Y. et al. Engineering 2D architectures toward high-performance microsupercapacitors. Adv. Mater. 31, 1802793 (2019).

4. Raccichini, R., Varzi, A., Passerini, S. \& Scrosati, B. The role of graphene for electrochemical energy storage. Nat. mater. 14, 271 (2015).

5. Nakhanivej, P. et al. Revealing molecular-level surface redox sites of controllably oxidized black phosphorus nanosheets. Nat. Mater. 18, 156-162 (2019).

6. Cao, X., Tan, C., Zhang, X., Zhao, W. \& Zhang, H. Solution-processed twodimensional metal dichalcogenide-based nanomaterials for energy storage and conversion. Adv. Mater. 28, 6167-6196 (2016).

7. Anasori, B., Lukatskaya, M. R. \& Gogotsi, Y. 2D metal carbides and nitrides (MXenes) for energy storage. Nat. Rev. Mater. 2, 16098 (2017).

8. Peng, L. et al. Holey two-dimensional transition metal oxide nanosheets for efficient energy storage. Nat. commun. 8, 15139 (2017).

9. Zhang, Y.-Z. et al. A simple approach to boost capacitance: flexible supercapacitors based on manganese oxides@MOFs via chemically induced in situ selftransformation. Adv. Mater. 28, 5242-5248 (2016). 
10. ten Elshof, J. E. \& Wang, Y. Advances in ink-jet printing of $\mathrm{MnO}_{2}$-nanosheet based pseudocapacitors. Small Methods 3, 1800318 (2019).

11. Lv, Z. et al. Editable supercapacitors with customizable stretchability based on mechanically strengthened ultralong $\mathrm{MnO}_{2}$ nanowire composite. Adv. Mater. 30, 1704531 (2018).

12. Lee, H. Y. \& Goodenough, J. B. Supercapacitor behavior with $\mathrm{KCl}$ electrolyte. J. Solid State Chem. 144, 220-223 (1999).

13. Kim, H. \& Popov, B. N. Synthesis and characterization of $\mathrm{MnO}_{2}$-based mixed oxides as supercapacitors. J. Electrochem. Soc. 150, D56-D62 (2003).

14. Wei, W., Cui, X., Chen, W. \& Ivey, D. G. Manganese oxide-based materials as electrochemical supercapacitor electrodes. Chem. Soc. Rev. 40, 1697-1721 (2011).

15. Kwon, K. D., Refson, K. \& Sposito, G. Defect-induced photoconductivity in layered manganese oxides: a density functional theory study. Phys. Rev. Lett. 100, 146601 (2008).

16. Wang, H. et al. Half-metallicity in single-layered manganese dioxide nanosheets by defect engineering. Angew. Chem., Int. Ed. 127, 1211-1215 (2015).

17. ten Elshof, J. E. Electronic band structure and electron transfer properties of twodimensional metal oxide nanosheets and nanosheet films. Curr. Opin. Solid State Mater. Sci. 21, 312-322 (2017).

18. Cai, J., Liu, J. \& Suib, S. L. Preparative parameters and framework dopant effects in the synthesis of layer-structure birnessite by air oxidation. Chem. Mater. 14, 2071-2077 (2002).

19. Kai, K. et al. One-pot synthesis of co-substituted manganese oxide nanosheets and physical properties of lamellar aggregates. Mater. Res. Bull. 47, 3855-3859 (2012).

20. Sakai, N., Fukuda, K., Ma, R. \& Sasaki, T. Synthesis and substitution chemistry of redox-active manganese/cobalt oxide nanosheets. Chem. Mater. 30, 1517-1523 (2018).

21. Kyeremateng, N. A., Brousse, T. \& Pech, D. Microsupercapacitors as miniaturized energy-storage components for on-chip electronics. Nat. Nanotechnol. 12, 7-15 (2016).

22. Pang, H., Zhang, Y., Lai, W.-Y., Hu, Z. \& Huang, W. Lamellar $\mathrm{K}_{2} \mathrm{Co}_{3}\left(\mathrm{P}_{2} \mathrm{O}_{7}\right)_{2} \cdot 2 \mathrm{H}_{2} \mathrm{O}$ nanocrystal whiskers: high-performance flexible all-solid-state asymmetric microsupercapacitors via inkjet printing. Nano Energy 15, 303-312 (2015). 
23. Xiong, Z. et al. A dynamic graphene oxide network enables spray printing of colloidal gels for high-performance micro-supercapacitors. Adv. Mater. 31, 1804434 (2019).

24. Lv, Z., Li, W., Yang, L., Loh, X. J. \& Chen, X. Custom-made electrochemical energy storage devices. ACS Energy Lett. 4, 606-614 (2019).

25. Zhang, Y.-Z. et al. Printed supercapacitors: materials, printing and applications. Chem. Soc. Rev. 48, 3229-3264 (2019).

26. Hu, G. et al. Functional inks and printing of two-dimensional materials. Chem. Soc. Rev. 47, 3265-3300 (2018).

27. Zhang, Y.-Z. et al. Flexible supercapacitors based on paper substrates: a new paradigm for low-cost energy storage. Chem. Soc. Rev. 44, 5181-5199 (2015).

28. Li, D., Lai, W.-Y., Zhang, Y.-Z. \& Huang, W. Printable transparent conductive films for flexible electronics. Adv. Mater. 30, 1704738 (2018).

29. Cheng, T. et al. Inkjet-printed flexible, transparent and aesthetic energy storage devices based on PEDOT:PSS/Ag grid electrodes. J. Mater. Chem. A 4, 1375413763 (2016).

30. Blöchl, P. E., Jepsen, O. \& Andersen, O. K. Improved tetrahedron method for Brillouin-zone integrations. Phys. Rev. B 49, 16223-16233 (1994).

31. Kresse, G. Efficient iterative schemes for ab initio total-energy calculations using a plane-wave basis set. Phys. Rev. B 54, 11169-11186 (1996).

32. Kresse, G. From ultrasoft pseudopotentials to the projector augmented-wave method. Phys. Rev. B 59, 1758-1775 (1999).

33. Perdew, J. Self-interaction correction to density-functional approximations for many-electron systems. Phys. Rev. B 23, 5048-5079 (1981).

34. Anisimov, V. I. Band theory and Mott insulators: Hubbard U instead of Stoner I. Phys. Rev. B 44, 943-954 (1991).

35. Miyake, T. \& Aryasetiawan, F. Screened Coulomb interaction in the maximally localized Wannier basis. Phys. Rev. B 77, 085122 (2008).

36. Kai, K. et al. Room-temperature synthesis of manganese oxide monosheets. J. Am. Chem. Soc. 130, 15938-15943 (2008). 
37. Yuan, H., Lubbers, R., Besselink, R., Nijland, M. \& ten Elshof, J. E. Improved Langmuir-Blodgett titanate films via in situ exfoliation study and optimization of deposition parameters. ACS Appl. Mater. Interfaces 6, 8567-8574 (2014).

38. Wang, Y., Zhang, Y.-Z., Dubbink, D. \& ten Elshof, J. E. Inkjet printing of $\delta-\mathrm{MnO}_{2}$ nanosheets for flexible solid-state micro-supercapacitor. Nano Energy 49, 481-488 (2018).

39. Omomo, Y., Sasaki, T., Wang, L. \& Watanabe, M. Redoxable nanosheet crystallites of $\mathrm{MnO}_{2}$ derived via delamination of a layered manganese oxide. J. Am. Chem. Soc. 125, 3568-3575 (2003).

40. Teng, Y. et al. Atomically thin defect-rich Fe-Mn-O hybrid nanosheets as high efficient electrocatalyst for water oxidation. Adv. Funct. Mater. 28, 1802463 (2018).

41. Hu, X. et al. 2D Fe-containing cobalt phosphide/cobalt oxide lateral heterostructure with enhanced activity for oxygen evolution reaction. Nano Energy 56, 109-117 (2019).

42. An, L. et al. Epitaxial heterogeneous interfaces on $\mathrm{N}-\mathrm{NiMoO}_{4} / \mathrm{NiS}_{2}$ nanowires/nanosheets to boost hydrogen and oxygen production for overall water splitting. Adv. Funct. Mater. 29, 1805298 (2019).

43. Zhao, Y. et al. Defect-engineered ultrathin $\delta-\mathrm{MnO}_{2}$ nanosheet arrays as bifunctional electrodes for efficient overall water splitting. Adv. Energy Mater., 7, 1700005 (2017).

44. Tsushima, S., Wahlgren, U. \& Grenthe, I. Quantum chemical calculations of reduction potentials of $\mathrm{AnO}_{2}{ }^{2+} / \mathrm{AnO}_{2}{ }^{+}(\mathrm{An}=\mathrm{U}, \mathrm{Np}, \mathrm{Pu}, \mathrm{Am})$ and $\mathrm{Fe}^{3+} / \mathrm{Fe}^{2+}$ couples. J. Phys. Chem. A 110, 9175-9182 (2006).

45. Sakai, N., Ebina, Y., Takada, K. \& Sasaki, T. Photocurrent generation from semiconducting manganese oxide nanosheets in response to visible light. J. Phys. Chem. B 109, 9651-9655 (2005).

46. Eastman, D. E., Himpsel, F. J. \& Knapp, J. A. Experimental exchange-split energyband dispersions for Fe, Co, and Ni. Phys. Rev. Lett. 45, 498-498 (1980).

47. Pech, D. et al. Ultrahigh-power micrometre-sized supercapacitors based on onionlike carbon. Nat. nanotechnol. 5, 651-654 (2010).

48. Xiao, X. et al. Fiber-based all-solid-state flexible supercapacitors for self-powered systems. ACS Nano 6, 9200-9206 (2012). 
49. El-Kady, M. F., Strong, V., Dubin, S. \& Kaner, R. B. Laser scribing of highperformance and flexible graphene-based electrochemical capacitors. Science $\mathbf{3 3 5}$, 1326-1330 (2012).

50. Shi, P. et al. Design of amorphous manganese oxide@multiwalled carbon nanotube fiber for robust solid-state supercapacitor. ACS Nano 11, 444-452 (2017).

51. Peng, Z. et al. Flexible boron-doped laser-induced graphene microsupercapacitors. ACS Nano 9, 5868-5875 (2015).

52. El-Kady, M. F. \& Kaner, R. B. Scalable fabrication of high-power graphene microsupercapacitors for flexible and on-chip energy storage. Nat. Commun. 4, 1475 (2013).

53. Stoller, M. D. \& Ruoff, R. S. Best practice methods for determining an electrode material's performance for ultracapacitors. Energy Environ. Sci. 3, 1294-1301 (2010). 


\section{APPENDICES}

\section{Deposition of Langmuir-Blodgett (LB) thin film}

To evaluate the thickness and lateral size of nanosheets, monolayer nanosheet thin films that were deposited on a silicon substrate by the Langmuir-Blodgett (LB) method were investigated by AFM measurement in standard tapping mode. The procedure of LB deposition is as following: a nanosheet ink was diluted in $500 \mathrm{~mL}$ doubly ionized (DI) water, then left standing for $2 \mathrm{~h}$. Then $50 \mathrm{~mL}$ of solution was taken from the middle or top part of the suspension and poured into a LB trough (KSV Minimicro, a Teflon trough with an active trough surface area of $100 \mathrm{~cm}^{2}, \mathrm{~L} 195 \times \mathrm{W} 51 \times \mathrm{D} 4 \mathrm{~mm}^{3}$ and a dipping well L10 $\times \mathrm{W} 28 \times \mathrm{D} 28$ $\mathrm{mm}^{3}$, trough volume $48 \mathrm{~cm}^{3}$ ). The nanosheet deposition process was conducted by compress the water-air interface at a rate of $3 \mathrm{~mm} \mathrm{~min}^{-1}$ by moving the Teflon barriers until a threshold surface pressure had been reached. The surface pressure was kept around $7 \mathrm{mN} / \mathrm{m}$ for all samples during deposition which was measured by a Wilhelmy plate attached to the KSV Minimicro frame.
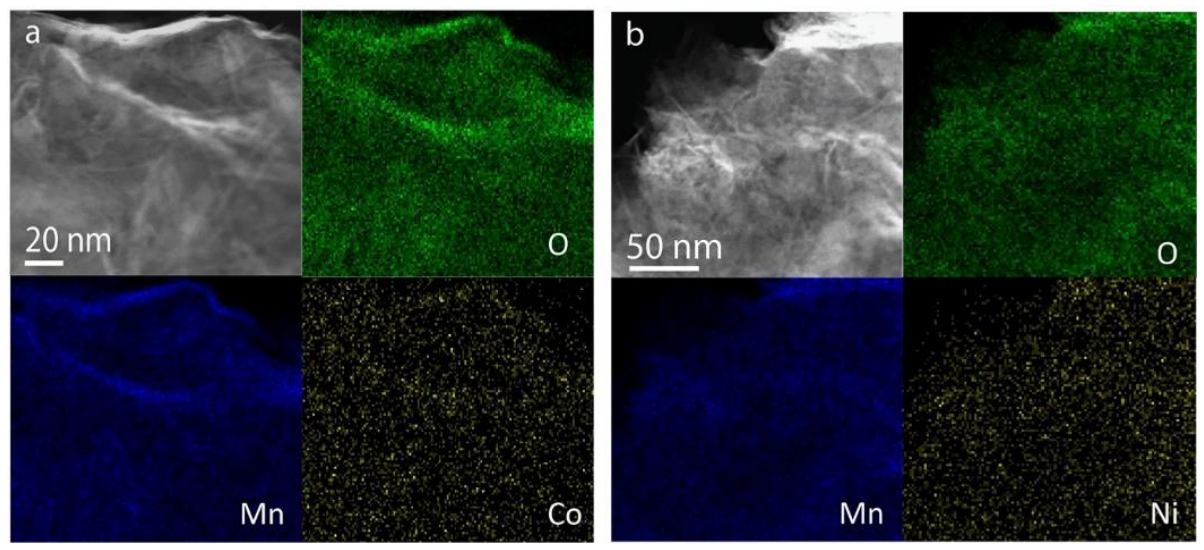

Figure S3.1 TEM and EDS elemental mapping of (a) $\mathrm{Co}-\mathrm{MnO}_{2}$ and (b) $\mathrm{Ni}-\mathrm{MnO}_{2}$ nanosheets. 

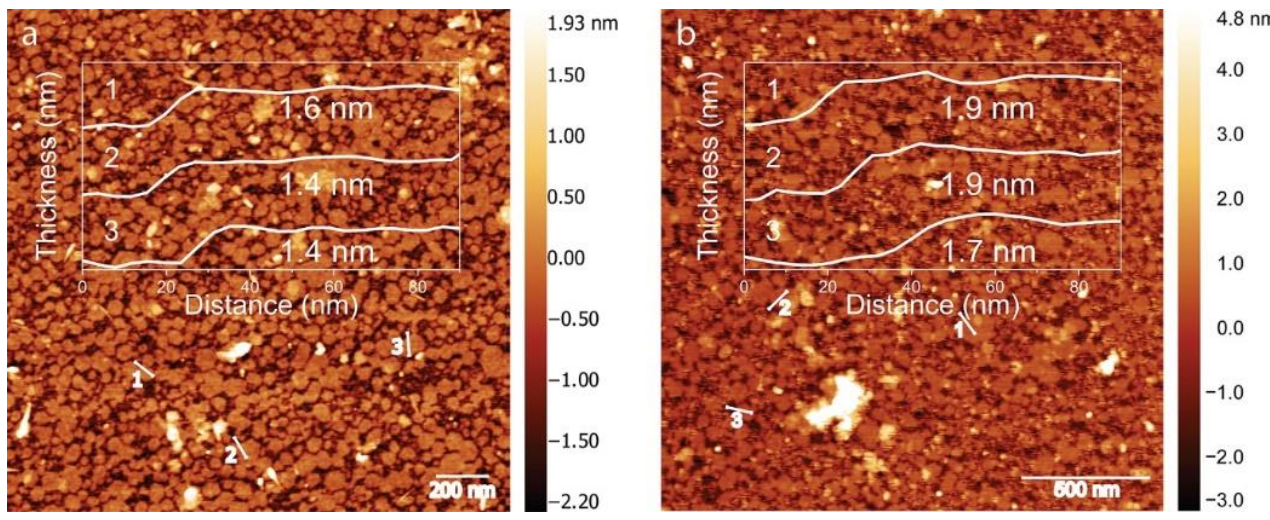

Figure S3.2 AFM images of (a) Co- $\mathrm{MnO}_{2}$ nanosheets and (b) $\mathrm{Ni}-\mathrm{MnO}_{2}$ nanosheets. The inner in a-b are the corresponding height profiles of nanosheets (the numbers of 1,2 and 3 correspond to the line scan number in AFM images).
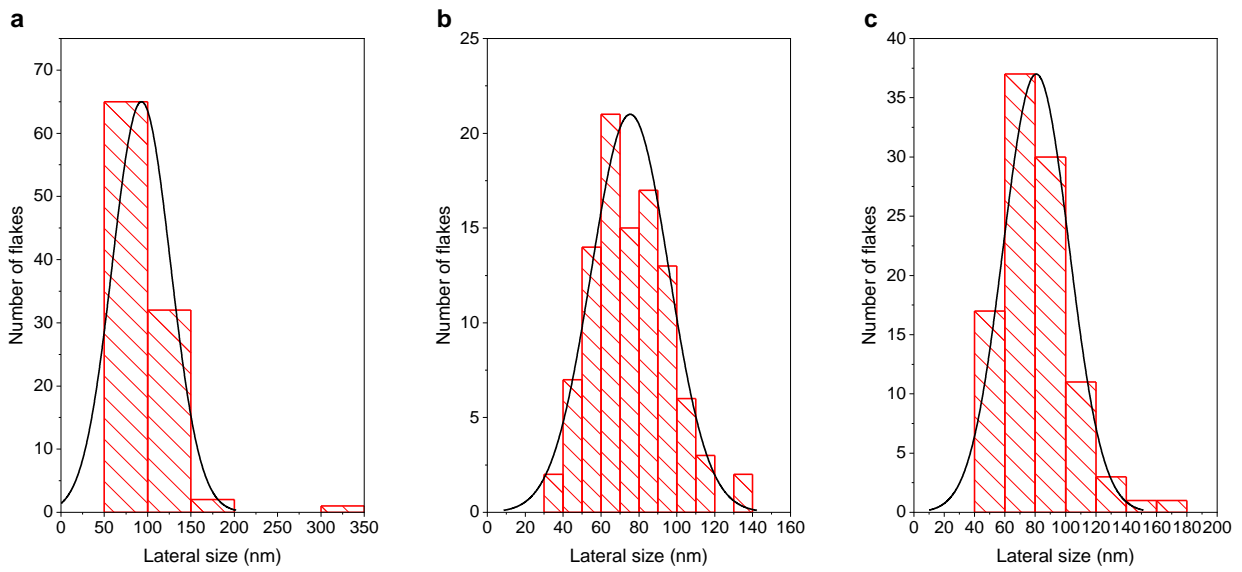

Figure S3.3 Lateral size distribution of (a) Fe, (b) $\mathrm{Co}$ and (c) $\mathrm{Ni}$-doped $\mathrm{MnO}_{2}$ nanosheets obtained by measuring 100 flakes on silicon by Langmuir-Blodgett (LB) deposition. 

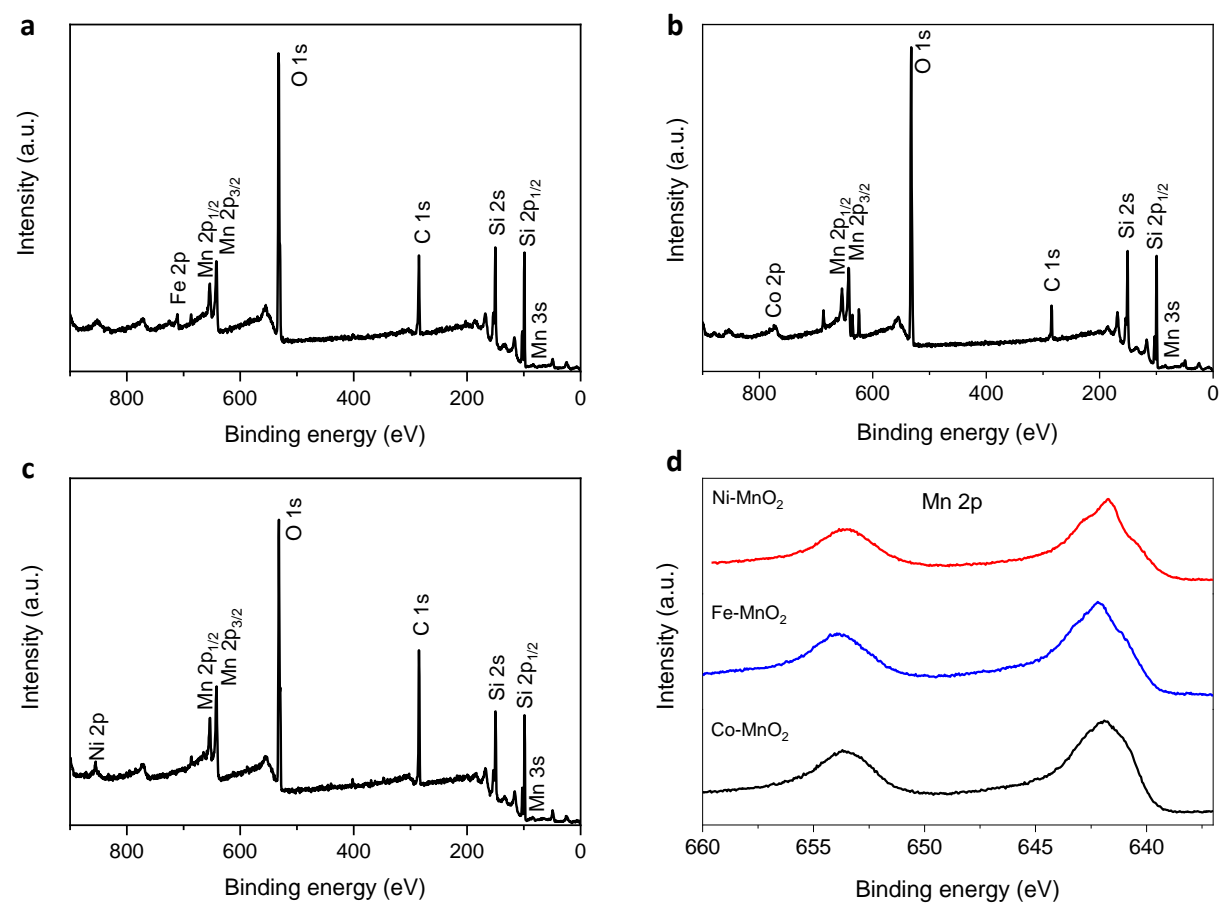

Figure S3.4 XPS survey scan spectrum of (a) $\mathrm{Fe}-\mathrm{MnO}_{2}$, (b) $\mathrm{Co}-\mathrm{MnO}_{2}$, and (c) $\mathrm{Ni}-\mathrm{MnO}_{2}$ nanosheets. (d) High resolution XPS of Mn $2 p$ in Fe, Co and Ni-doped $\mathrm{MnO}_{2}$ nanosheets. The $\Delta \mathrm{E}$ of Mn $3 s$ peaks of $\mathrm{Fe}$, Co and Ni-doped $\mathrm{MnO}_{2}$ are different, which indicates that the ratio of $\mathrm{Mn}^{3+}$ to $\mathrm{Mn}^{4+}$ is different in $\mathrm{Fe}$, Co and Ni-doped $\mathrm{MnO}_{2}$. Similarly, the $\mathrm{Mn} 2 p_{3 / 2}$ binding energy positons are also different for $\mathrm{MnO}_{2}$ and $\mathrm{Mn}_{2} \mathrm{O}_{3}$. Therefore, the Mn $2 p_{3 / 2}$ binding energy positions of $\mathrm{Fe}$, Co and Ni-doped $\mathrm{MnO}_{2}$ nanosheets can also differ. 


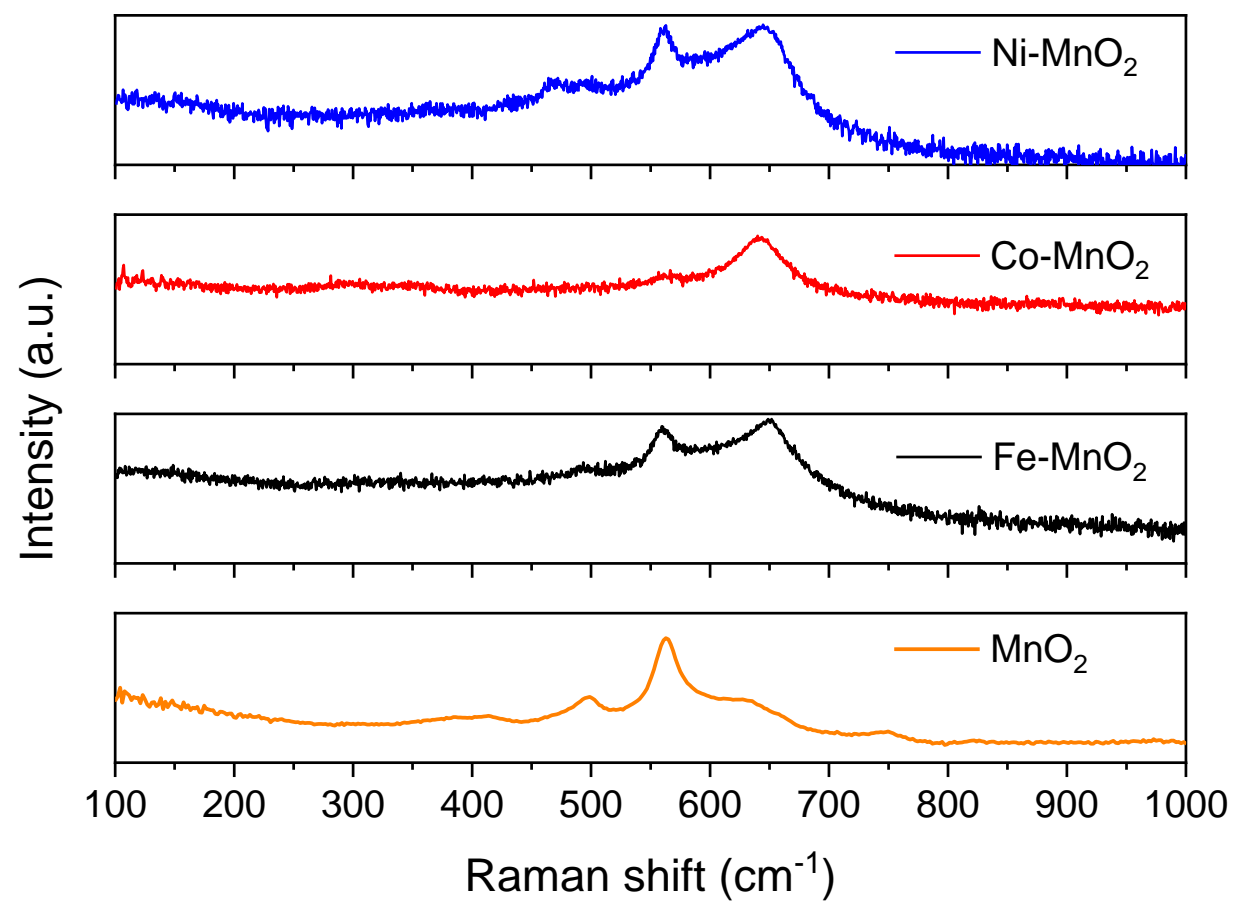

Figure S3.5 Raman spectrums of Fe-, Co- and Ni-doped $\mathrm{MnO}_{2}$ and undoped $\mathrm{MnO}_{2}$ nanosheets.

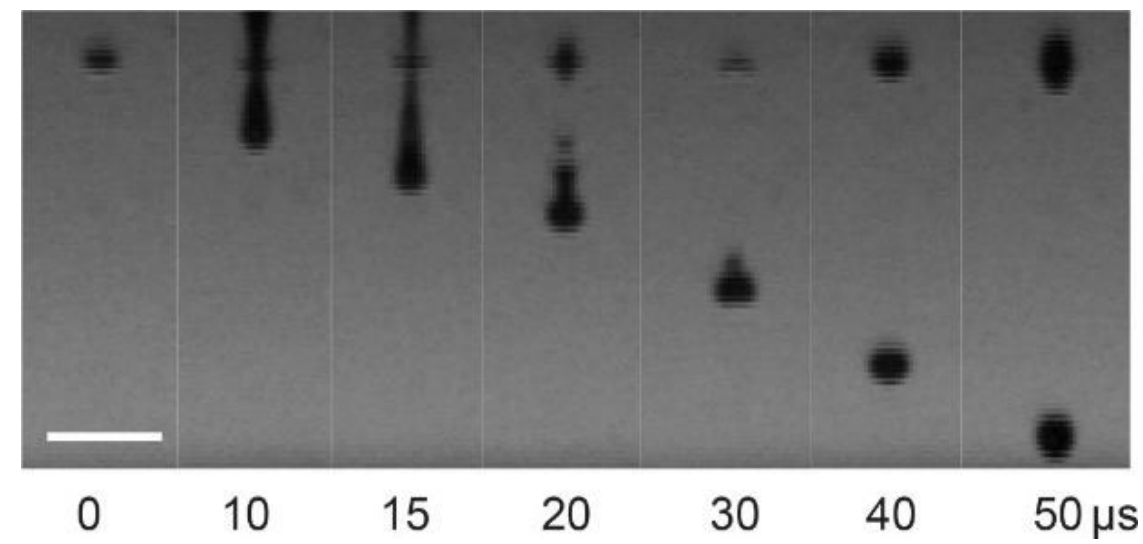

Figure S3.6 Stroboscopic images of $\mathrm{Fe}-\mathrm{MnO}_{2}$ ink droplet formation versus time observed from inkjet printer camera. The scale bar is $100 \mu \mathrm{m}$. 

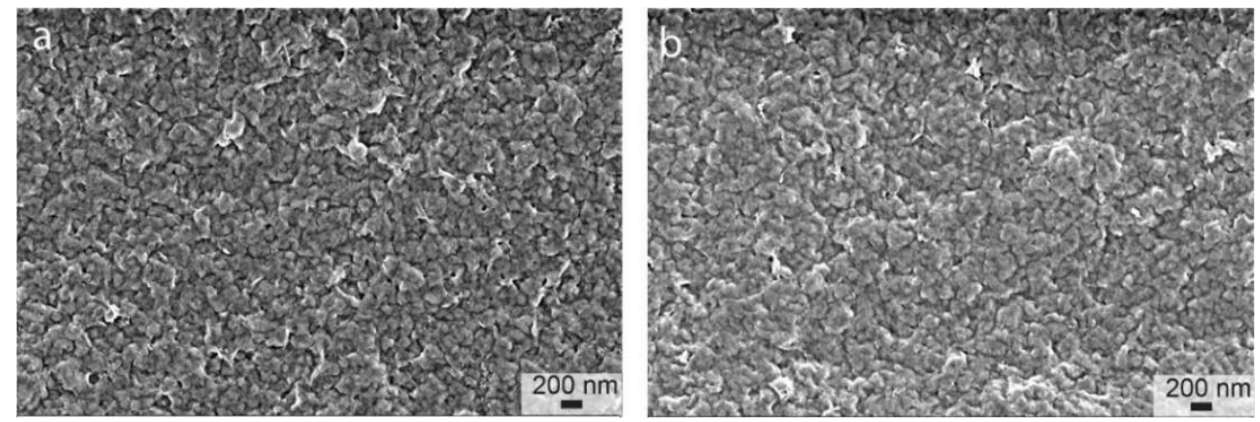

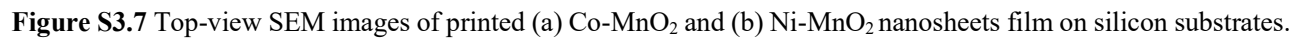

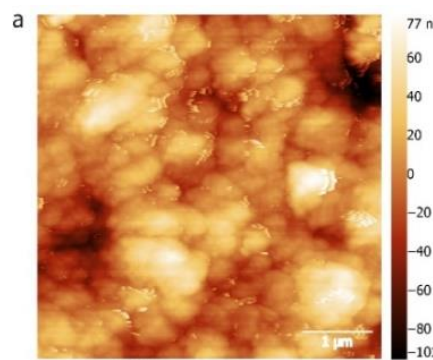

$\mathrm{RMS}=26 \pm 7 \mathrm{~nm}$

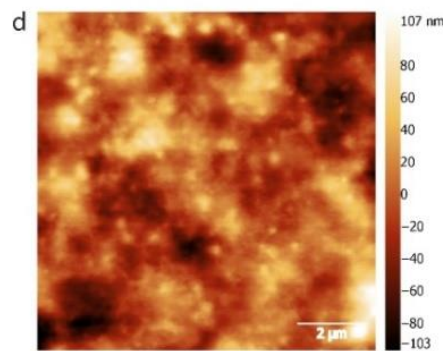

$\mathrm{RMS}=32 \pm 6 \mathrm{~nm}$

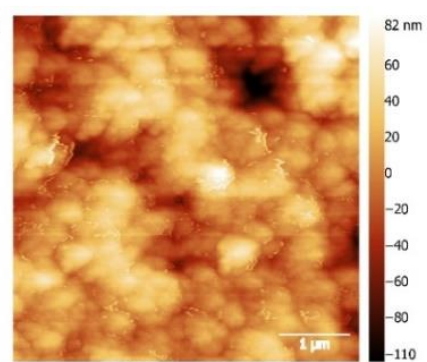

$\mathrm{RMS}=26 \pm 8 \mathrm{~nm}$

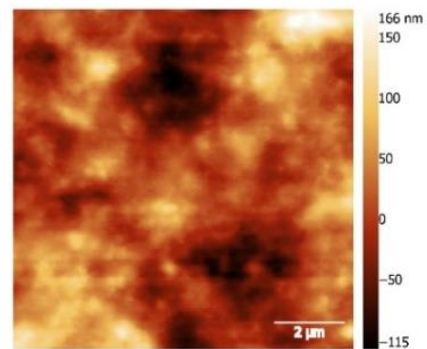

$\mathrm{RMS}=43 \pm 13 \mathrm{~nm}$

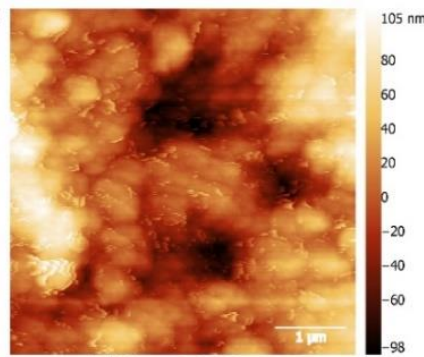

$\mathrm{RMS}=36 \pm 8 \mathrm{~nm}$

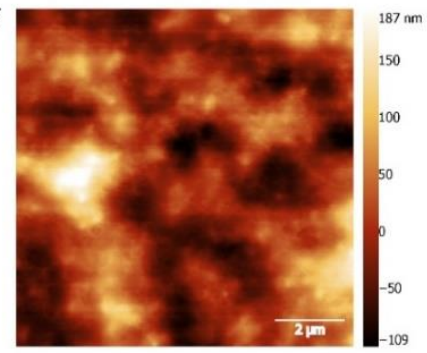

$\mathrm{RMS}=51 \pm 12 \mathrm{~nm}$

Figure S3.8 $5 \mu$ m by $5 \mu$ m AFM images of inkjet printed (a) $\mathrm{Fe}-\mathrm{MnO}_{2}$, (b) $\mathrm{Co}-\mathrm{MnO}_{2}$ and (c) $\mathrm{Ni}-\mathrm{MnO}_{2}$ nanosheets films on silicon substrates. $10 \mu \mathrm{m}$ by $10 \mu \mathrm{m}$ AFM images of inkjet printed (c) $\mathrm{Fe}-\mathrm{MnO}_{2}$, (d) $\mathrm{Co}-\mathrm{MnO}_{2}$ and (e) Ni$\mathrm{MnO}_{2}$ nanosheets films on silicon substrates. 
Chapter 3
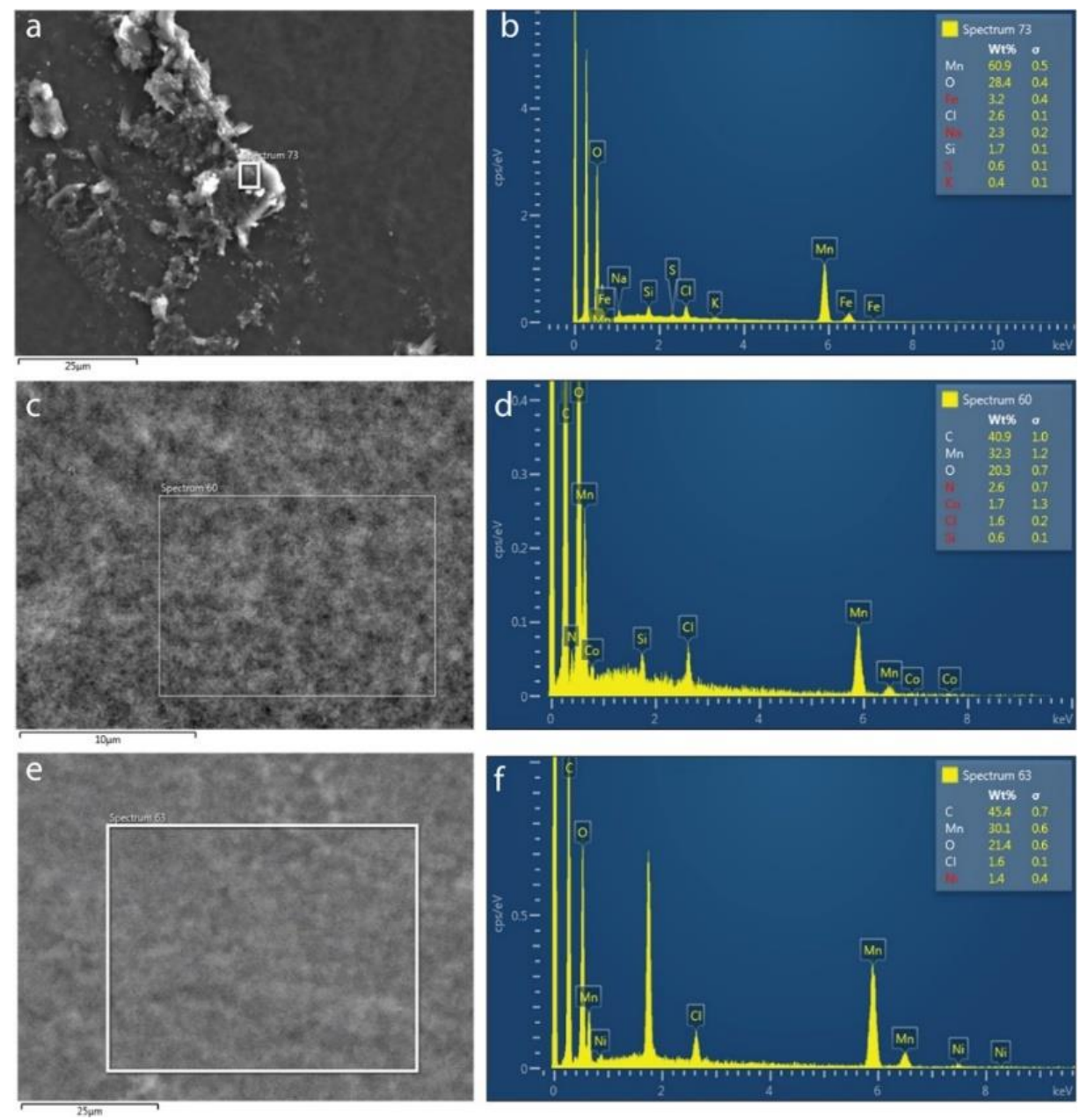

Figure S3.9 EDS patterns images of printed (a-b) $\mathrm{Fe}-\mathrm{MnO}_{2}$, (c-d) $\mathrm{Co}-\mathrm{MnO}_{2}$ and (e-f) $\mathrm{Ni}-\mathrm{MnO}_{2}$ samples. 


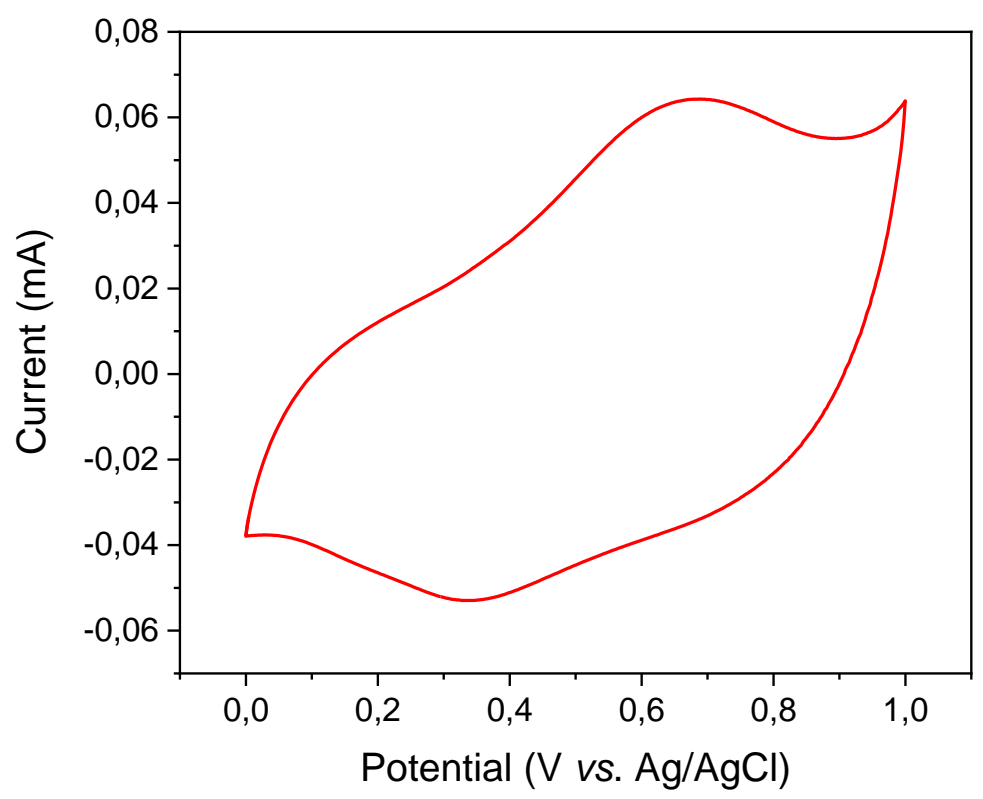

Figure S3.10 CV curve of Fe- $\mathrm{MnO}_{2}$ electrode in $5 \mathrm{M} \mathrm{LiCl}$ electrolyte at $5 \mathrm{mV} \mathrm{s}^{-1}$.
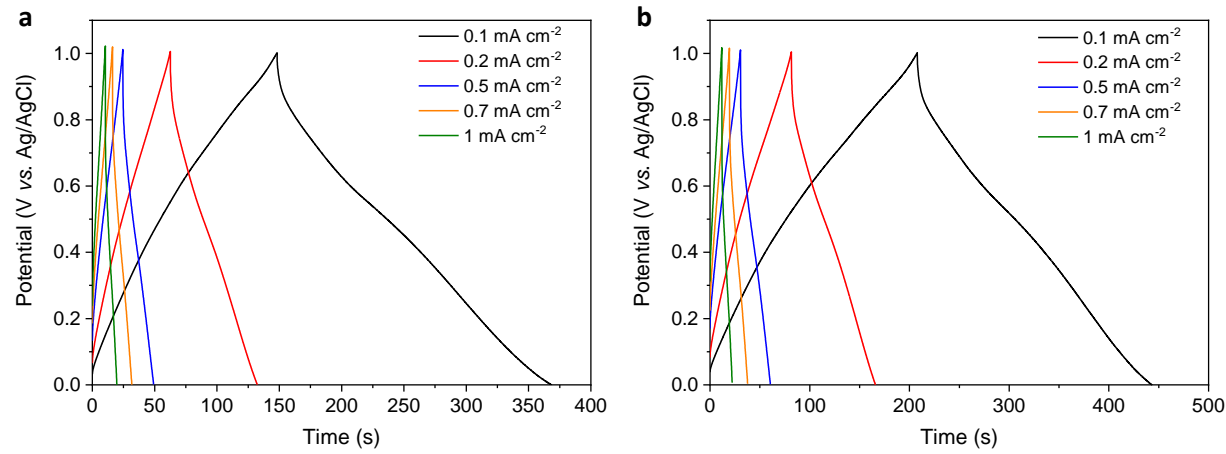

Figure S3.11 GCD of (a) Co, (b) Ni-doped $\mathrm{MnO}_{2}$ electrodes at different current densities. 


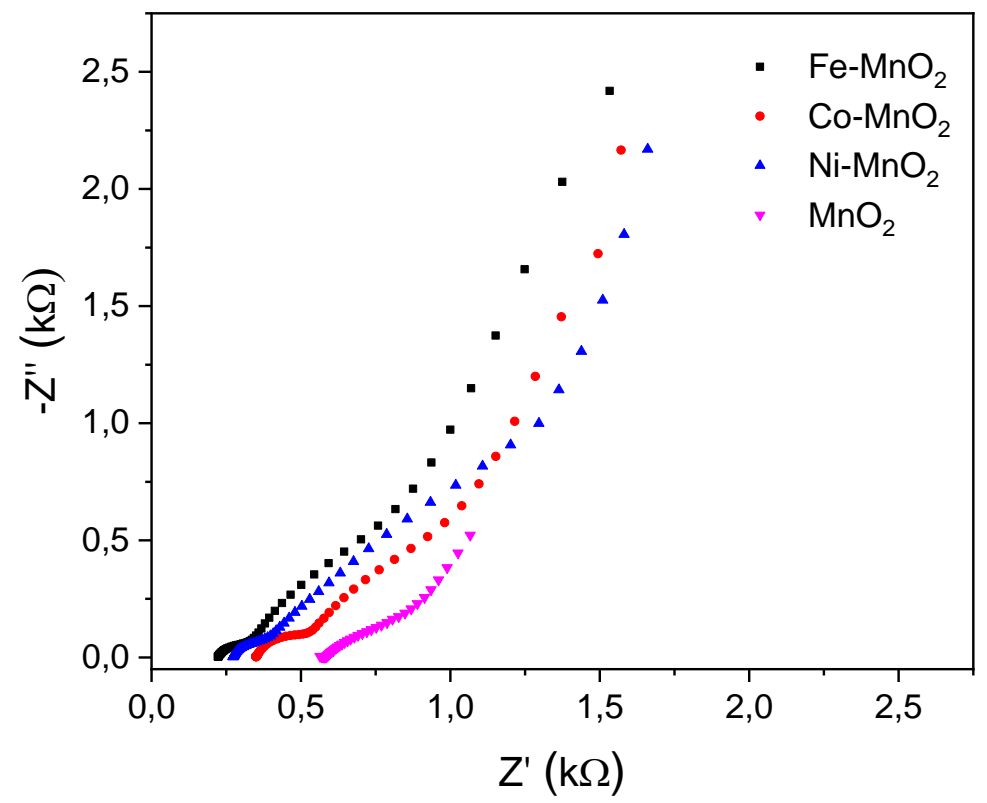

Figure S3.12 Nyquist plots for different electrodes.
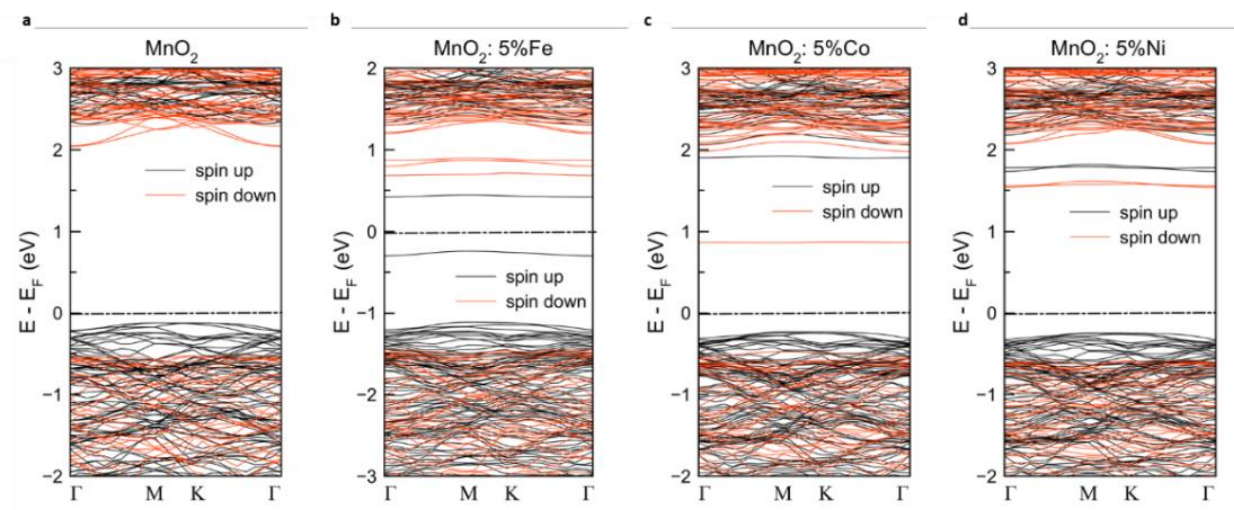

Figure S3.13 Band structure of (a) pristine, (b) 5\% Fe, (c) 5\% Co and (d) 5\% Ni-doped $\mathrm{MnO}_{2}$ nanosheets. The dashed lines specify the Fermi level $E_{\mathrm{F}}$. 
a

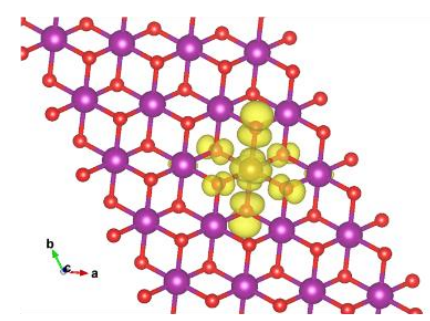

c

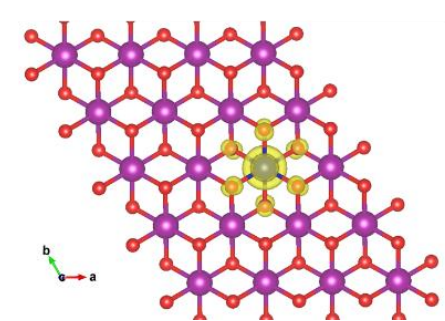

b
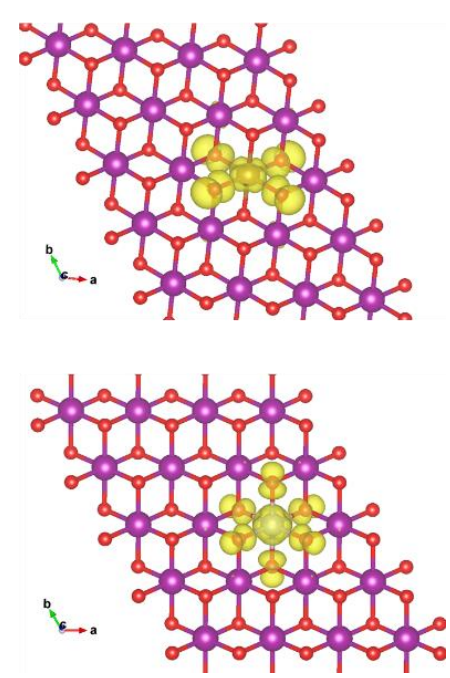

Figure S3.14 Partial charge density of in-gap impurity states of (a-b) $\mathrm{Fe}-\mathrm{MnO}_{2}$, (c) $\mathrm{Co}-\mathrm{MnO}_{2}$ and (d) $\mathrm{Ni}-\mathrm{MnO}_{2}$ nanosheets. The purple and red atoms represent manganese and oxygen atoms, respectively. The isosurface levels are 0.002 e $\AA^{-3}$.
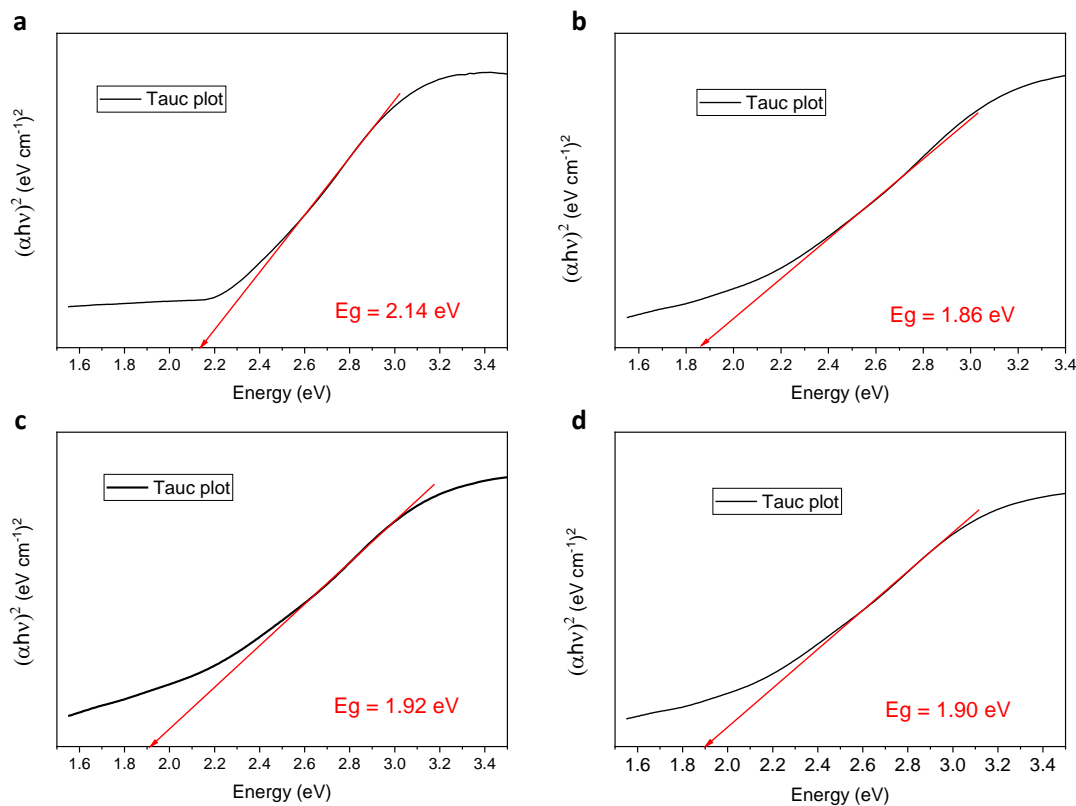

Figure S3.15 Tauc plots form UV-Vis analysis of (a) undoped $\mathrm{MnO}_{2}$, (b) $\mathrm{Fe}-\mathrm{MnO}_{2}$, (c) $\mathrm{Co}-\mathrm{MnO}_{2}$ and (d) $\mathrm{Ni}-\mathrm{MnO}_{2}$ nanosheets. 

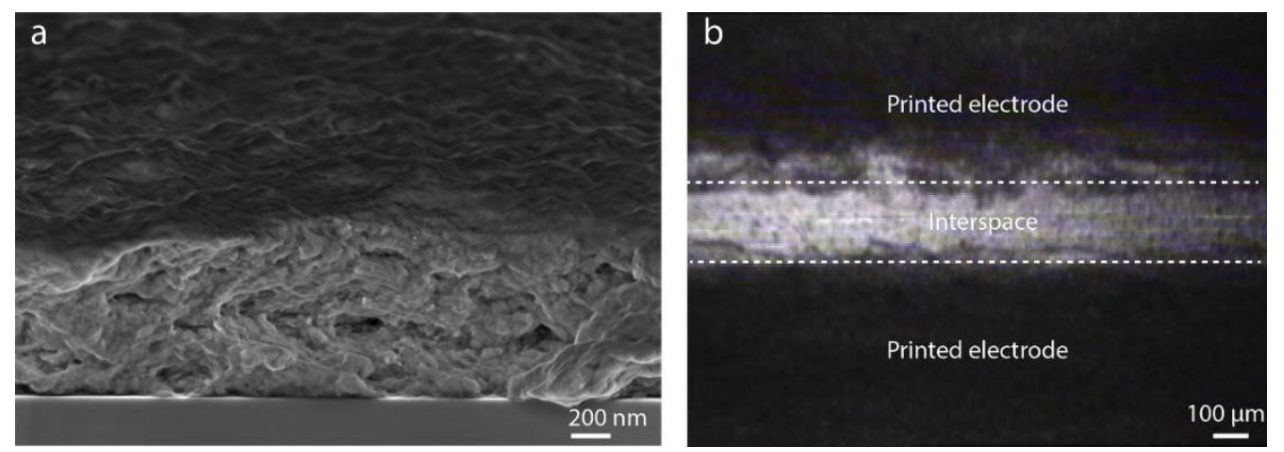

Figure S3.16 (a) Cross-sectional SEM image of printed $\mathrm{Fe}-\mathrm{MnO}_{2}$ electrode. (b) Optical image of printed electrodes and interspace between them. The dashed lines indicate roughly the interfacial zone between electrodes and interspace.

Table S3.1 Comparison of the series resistance $\left(\mathrm{R}_{1}\right)$ and charge transfer resistance $\left(\mathrm{R}_{2}\right)$ of pristine, $\mathrm{Fe}$, $\mathrm{Co}$ and $\mathrm{Ni}$-doped $\mathrm{MnO}_{2}$ nanosheets electrodes.

\begin{tabular}{ccccc}
\hline Materials & $\mathrm{R}_{1}(\Omega)$ & $\mathrm{R}_{2}(\Omega)$ & $\mathrm{Q}_{2}\left(\mu \mathrm{Mho}{ }^{*} \mathrm{~s}^{\wedge}\right)$ & $\mathrm{n}$ \\
\hline $\mathrm{Fe}-\mathrm{MnO}_{2}$ & 222 & 148 & 115 & 0.996 \\
$\mathrm{Co-} \mathrm{MnO}_{2}$ & 350 & 260 & 141 & 0.996 \\
$\mathrm{Ni}^{-\mathrm{MnO}_{2}}$ & 272 & 170 & 97 & 0.996 \\
Pristine $\mathrm{MnO}_{2}$ & 582 & 606 & 615 & 0.993 \\
\hline
\end{tabular}




\section{Chapter 4}

\section{Tunable Capacitance in All-Inkjet-Printed Nanosheet Heterostructures}

Heterostructures constructed from two-dimensional building blocks have shown promise for field-effect transistors, memory devices, photosensors and other electronic applications. 2D nanosheet crystals can be constructed into multilayer heterostructures using layer-by-layer methods, but that method cannot be used to fabricate large-scale and thick heterostructures, due to the time-consuming nature and low efficiency of the process. An alternative approach to deposit different two-dimensional materials is by inkjet printing. Here we show the fabrication of a nanosheet supercapacitor by inkjet printing $\mathrm{Ti}_{3} \mathrm{C}_{2} \mathrm{~T}_{x}$ MXene nanosheets as electrodes, and graphene oxide nanosheets as solid-state electrolyte. The free water molecules trapped between graphene oxide sheets facilitate proton movement through the layered solid electrolyte. The as-made supercapacitor shows high areal capacitance, good cycling stability and high areal energy and power densities comparable with existing printed supercapacitors. Moreover, the specific capacitance can be increased further by addition of liquid electrolytes.

This chapter has been submitted as:

Wang, Y.; Mehrali, M.; Zhang, Y.-Z.; Timmerman, M. A.; Boukamp, B. A.; Xu, P.-Y.; ten Elshof, J. E., Tunable capacitance in all-inkjet-printed nanosheet heterostructures. Preprint at http:// https://arxiv.org/abs/2004.03350 (2020). 


\subsection{Introduction}

Two-dimensional (2D) heterostructures with vertical stacking configurations are useful for a vast range of applications due to their exciting properties, such as superconductivity, magnetism and energy storage capability ${ }^{1,2}$. Mechanical exfoliation of three-dimensional layered compounds followed by dry transfer of each $2 \mathrm{D}$ nanosheet onto a substrate is still the main technique for vertical 2D heterostructure fabrication ${ }^{3}$. The advantage of this technique is that atomically thin high-quality heterostructures can be realized on small scale.

2D transition metal carbides or nitrides (MXenes) with general formula $\mathrm{M}_{n+1} \mathrm{X}_{n} \mathrm{~T}_{x}(n=1,2$, $3,4)$, where $M$ is an early transition metal, $X$ is a carbon and/or nitrogen and $T_{x}$ are surface terminal groups like $-\mathrm{F},-\mathrm{O}$, or $-\mathrm{OH}$, have been attracting tremendous attention recently due to their outstanding chemical and physical properties ${ }^{4,5}$. MXenes with atomic thickness and high electrical conductivity have been widely studied for application in hydrogel sensors ${ }^{6}$, solar cells ${ }^{7}$ and supercapacitors $(\mathrm{SCs})^{8,9}$. MXenes have been combined with other 2D materials into multi-material structures with tunable properties and functionalities, showing promise for energy storage applications ${ }^{10}$. On the other hand, hydrated graphene oxide (GO) nanosheets are electrically insulating but exhibit high ionic conductivity, suggesting their potential as solid-state electrolyte and separator ${ }^{11}$.

MXene and GO are normally dispersed in a solvent like water, but traditional mechanical exfoliation and drying transfer techniques cannot be applied to fabricate MXene based heterostructures on large scale. Solution processing methods such as spray coating and vacuum filtration have been attempted, but these offer poor control over interface and surface roughness, resulting in poor device performance ${ }^{10}$. Inkjet printing, a simple, low-cost and versatile technique, provides an alternative route to the fabrication of large-scale vertical heterostructures with controlled thickness, interface and roughness ${ }^{12}$. Recently, various heterostructure devices based on printed $2 \mathrm{D}$ materials such as field-effect transistors ${ }^{13}$, capacitors $^{14}$, photosensors and memory devices ${ }^{12}$ have been demonstrated. However, realizing well controlled and sharp interfaces still presents a significant challenge for printed heterostructures. Full control over the heterostructure interface is key to achieving high performance, which includes avoiding redispersion of nanosheets from the interface upon deposition of a subsequent layer. The preparation of non-toxic, stable and printable 2D inks is another critical issue for inkjet printing.

Owing to the wide range of physical properties present in $2 \mathrm{D}$ materials ${ }^{15}$, we demonstrate here that a combination of 2D materials can be used to realize an all-solid-state supercapacitor, without any liquid or gel electrolyte present in the system. In this work, we used a waterbased additive-free MXene ink to inkjet print electrodes and current collectors on polyimide substrates, and a water-based GO ink to inkjet-print the solid-state electrolyte. Both sandwiched supercapacitors (SSCs) and micro-supercapacitor devices (MSCs) were printed 
on flexible polyimide substrates (Figure 4.1). The SSCs achieved specific areal capacitances $\left(C_{\mathrm{A}}\right)$ up to $9.8 \mathrm{mF} \mathrm{cm}^{-2}$ at a current density of $40 \mu \mathrm{A} \mathrm{cm}^{-2}$. Addition of aqueous electrolytes led to enhancement of $C_{\mathrm{A}}$, due to the improved ionic conductivity of the electrolyte resulting from the presence of additional ions and a liquid phase.

\subsection{Experimental Section}

\subsubsection{Preparation of MXene ink}

Titanium carbide $\left(\mathrm{Ti}_{3} \mathrm{C}_{2} \mathrm{~T}_{x}\right)$ MXene was synthesized following a mild etching method as outlined elsewhere. ${ }^{16}$ Typically, the etchant solution was prepared by dissolving $3.2 \mathrm{~g}$ of lithium fluoride (LiF, Sigm-Aldrich, -300 mesh powder, $98.5 \%$ ) in $40 \mathrm{~mL}$ of $9 \mathrm{M} \mathrm{HCl}$ (SigmAldrich, $37 \%$ solution in water). Subsequently, $2 \mathrm{~g}$ of sieved $\mathrm{Ti}_{3} \mathrm{AlC}_{2}$ powder (400 mesh) was slowly added to the etchant solution over the course of $10 \mathrm{~min}$ and the reaction temperature was kept at $35{ }^{\circ} \mathrm{C}$. After reaction for $24 \mathrm{~h}$, the resultant was washed with deionized water repeatedly and delaminated manually by hand shaking agitation to obtain $\mathrm{Ti}_{3} \mathrm{C}_{2} \mathrm{~T}_{x}$ MXene suspension. The prepared solution was stored in a nitrogen-sealed vial and used as the MXene ink.

\subsubsection{Preparation of graphene oxide (GO) nanosheets ink}

Graphite oxide was synthesized from natural graphite (Nord-Min 802, Chemical Schmits Solutions) by a modified Hummers method. ${ }^{17}$ Graphite $(2 \mathrm{~g})$ was added to $50 \mathrm{~mL}$ concentrated sulfuric acid (Fluka) in a $1000 \mathrm{~mL}$ flask under stirring in an ice bath for $2 \mathrm{~h}$. Then $7 \mathrm{~g}$ potassium permanganate (Merck) was slowly added to the suspension under vigorous stirring to keep the temperature of the mixture under $10{ }^{\circ} \mathrm{C}$. The mixture was transferred to a $35^{\circ} \mathrm{C}$ oil bath under stirring for $20 \mathrm{~h}$, yielding a thick paste. After the mixture had cooled down to room temperature, $100 \mathrm{~mL}$ DI water was slowly added with vigorous stirring for $2 \mathrm{~h}$ while keeping the flask in an ice bath. An additional $500 \mathrm{~mL}$ DI water was added, followed by addition of $15 \mathrm{~mL} \mathrm{H}_{2} \mathrm{O}_{2}$ (30 wt $\%$, Aldrich) until no further bubbles came out. The mixture was washed by 1: $10 \mathrm{HCl}(37 \%$, Acros organics) solution $(250 \mathrm{~mL})$ to remove metal ions, and subsequently with DI water to a $\mathrm{pH}$ around 6 . The resulting solid was freeze dried. The freeze dried graphite oxide powder was dispersed in DI water by ultrasonication for $2 \mathrm{~h}$ to get a GO suspension. To increase the GO concentration, the GO suspension was centrifuged at $15000 \mathrm{~g}$ for $1 \mathrm{~h}$. The collected sediment was re-dispersed in printing solvent containing $0.06 \mathrm{wt} \%$ Triton X-100 (Sigma-Aldrich) and 1:10 propylene glycol (Sigma-Aldrich) : water by mass.

\subsubsection{Inkjet printing}

All patterns and devices were inkjet printed by a Dimatix DMP-2800 inkjet printer (Fujifilm Dimatix), which was equipped with a $10 \mathrm{pL}$ cartridge (DMC-11610). To perform AFM measurements on single droplets, the MXene and GO inks were inkjet printed on $\mathrm{Si} / \mathrm{SiO}_{2}$ 
with a drop spacing of $80 \mu \mathrm{m}$ at $30{ }^{\circ} \mathrm{C}$. For the electrical conductivity measurements, the MXene ink was printed at $30{ }^{\circ} \mathrm{C}$ on $\mathrm{Si} / \mathrm{SiO}_{2}$ as a thin film with size $5 \mathrm{~mm}$ by $5 \mathrm{~mm}$ and varying numbers of layers at a drop spacing of $20 \mu \mathrm{m}$.

To fabricate all-inkjet-printed solid-state sandwiched supercapacitors, the MXene ink was first printed at $30{ }^{\circ} \mathrm{C}$ as bottom electrode on polyimide substrate with a drop spacing of 20 $\mu \mathrm{m}$ followed by drying at $50^{\circ} \mathrm{C}$ for $1 \mathrm{~h}$. Then GO ink was printed on top of MXene electrode with a drop spacing of $20 \mu \mathrm{m}$ at $30^{\circ} \mathrm{C}$ followed by drying at $50^{\circ} \mathrm{C}$ for $1 \mathrm{~h}$. Finally, the MXene ink was printed at $30^{\circ} \mathrm{C}$ on $\mathrm{GO}$ electrolyte as top electrode with a drop spacing of $20 \mu \mathrm{m}$. It is worth noting that the GO electrolyte area is larger than the MXene electrode to prevent short-circuiting.

To make all-inkjet-printed solid-state micro-supercapacitors, the MXene ink was printed with an interdigitated configuration on polyimide substrate as interdigitated electrodes at $30{ }^{\circ} \mathrm{C}$ with different layers at a drop spacing of $20 \mu \mathrm{m}$ using 2 nozzles, followed by drying at $50{ }^{\circ} \mathrm{C}$ for $1 \mathrm{~h}$. Then, the GO ink was printed at $30^{\circ} \mathrm{C}$ on top of the MXene electrodes at a drop spacing of $20 \mu \mathrm{m}$.

\subsubsection{Electrochemical characterization}

All electrochemical characterizations were conducted on an Autolab workstation (PGSTAT128 N). Both micro-supercapacitors and sandwiched supercapacitors were characterized in a two-electrode configuration. Electrochemical impedance spectroscopy was performed by applying an $\mathrm{AC}$ voltage of $10 \mathrm{mV}$ amplitude in the frequency range from 0.01 $\mathrm{Hz}$ to $10 \mathrm{kHz}$.

\subsubsection{Materials characterization}

X-ray diffraction (XRD) analysis was done with a PANalytical X'Pert Pro with $\mathrm{Cu} \mathrm{K} \alpha$ radiation $(\lambda=0.15405 \mathrm{~nm})$. Atomic Force Microscopy (AFM) (Veeco Dimension Icon) was conducted in standard tapping mode. The AFM data were analyzed by Gwyddion (version 2.47) software. X-ray photoelectron spectroscopy (XPS) was conducted using an Omicron Nanotechnology GmbH (Oxford Instruments) surface analysis system with a photon energy of $1486.7 \mathrm{eV}$ (Al K $\alpha$ X-ray source) with a scanning step size of $0.1 \mathrm{eV}$. The pass energy was set to $20 \mathrm{eV}$. The spectra were corrected using the binding energy of $\mathrm{C} 1 \mathrm{~s}$ of the carbon residual on nanosheets as a reference. Raman spectroscopy was performed on a Bruker Senterra Raman spectrometer using a $532 \mathrm{~nm}$ laser under ambient conditions. High resolution scanning electron microscopy (HRSEM; Zeiss MERLIN) was performed to acquire information of printed MXene and GO films. The surface tension of the inks was measured by a contact angle system OCA (Data Physics Corporation). The viscosity of the inks was determined by an Automated Microviscometer AMVn (Anton Paar GmbH).

The electrical conductivity of printed MXene films on $\mathrm{Si} / \mathrm{SiO}_{2}$ was measured in a Van der 
Pauw geometry by Physical Properties Measurement System (PPMS) at 300 K. Copper wires were bonded on four corners of printed MXene films by silver paste. $R_{\mathrm{s}}$ was calculated from the following Equation (1):

$R_{\mathrm{s}}=\pi R / \ln 2$

The specific areal capacitance $\left(C_{\mathrm{A}}\right)$ of devices were calculated from the GCD curves by using Equation (2):

$C_{\mathrm{A}}=[I /(\mathrm{d} V / \mathrm{d} t)] / A_{\text {electrode }}$

where $I$ is the discharge current, $\mathrm{d} V / \mathrm{d} t$ is the slope of discharge curve, and $A_{\text {electrode }}$ refers to the total geometrical surface area of the device including the electrodes and the gap between the electrodes.

The areal energy densities $\left(E_{\mathrm{A}}, \mu \mathrm{Wh} \mathrm{cm}^{-2}\right)$ and power densities $\left(P_{\mathrm{A}}, \mu \mathrm{W} \mathrm{cm}{ }^{-2}\right)$ were calculated from equations (3) and (4)

$E_{\mathrm{A}}=C_{\mathrm{A}, \text { device }} V^{2} /(2 \times 3.6)$

$P_{\mathrm{A}}=3600 \times E_{\mathrm{A}} / \Delta t$

Where $\Delta t$ refers to discharge time.

\subsection{Results and discussion}

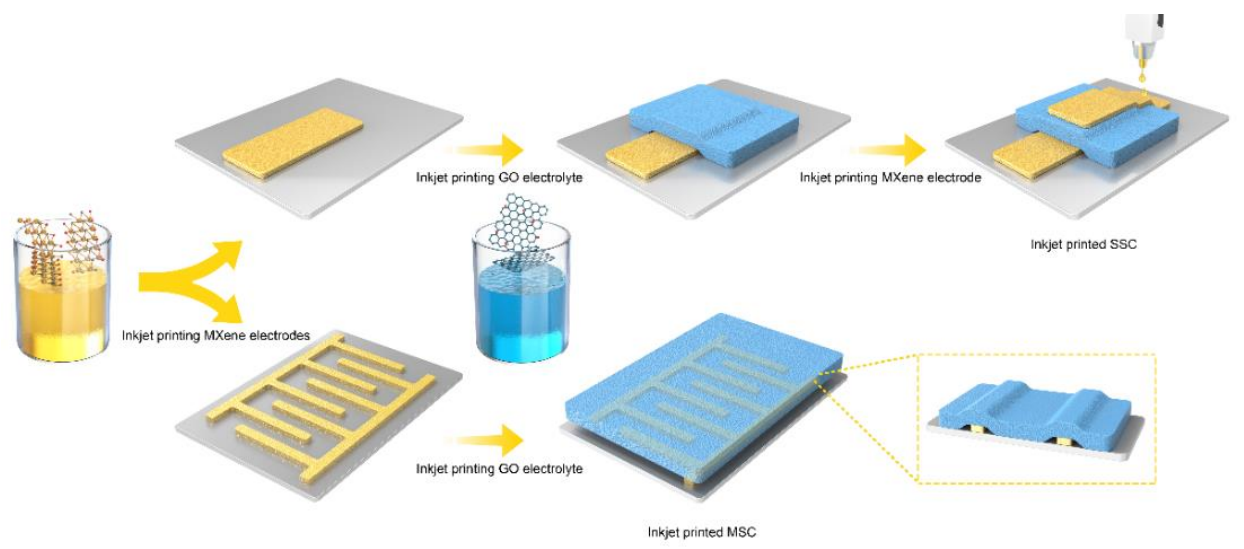

Figure 4.1 Schematic illustration of all-inkjet-printing-based heterostructure SSC (top) and MSC (bottom) supercapacitors. Water-based additive-free MXene ink was first inkjet printed into thin films and interdigitated configurations as electrodes, followed by inkjet printing a water-based GO ink on top of interdigitated MXene electrodes to form an all-solid-state MSC (bottom). A second MXene electrode was inkjet printed on top of the solidstate GO electrolyte to complete the fabrication process of an all-solid-state SSC (above). 
a

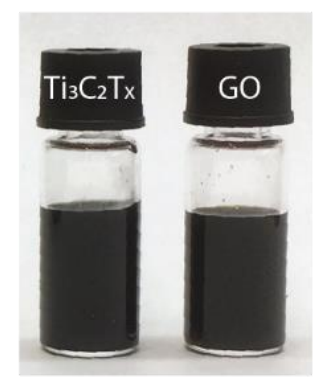

c

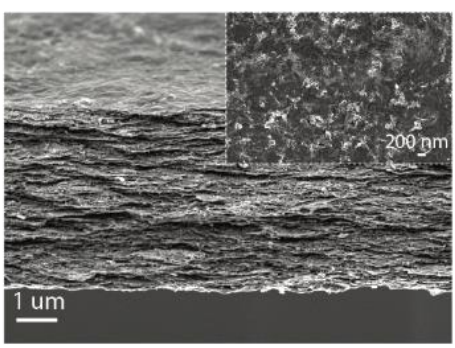

b

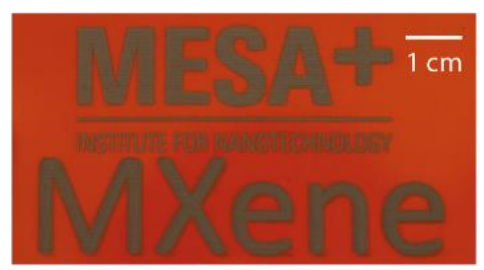

d

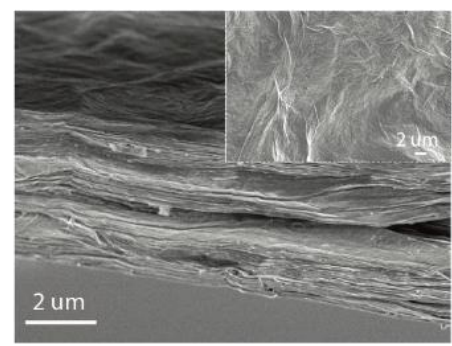

Figure 4.2 Characterization of MXene and GO nanosheets. (a) Optical image of water-based MXene and GO inks. (b) Inkjet printed "MESA+ INSTITUTE FOR NANOTECHNOLOGY" logo and word "MXene" using MXene ink on polyimide substrate with multiple printing layers. Cross-sectional SEM image of (c) printed MXene and (d) GO films on $\mathrm{Si} / \mathrm{SiO}_{2}$ substrates.
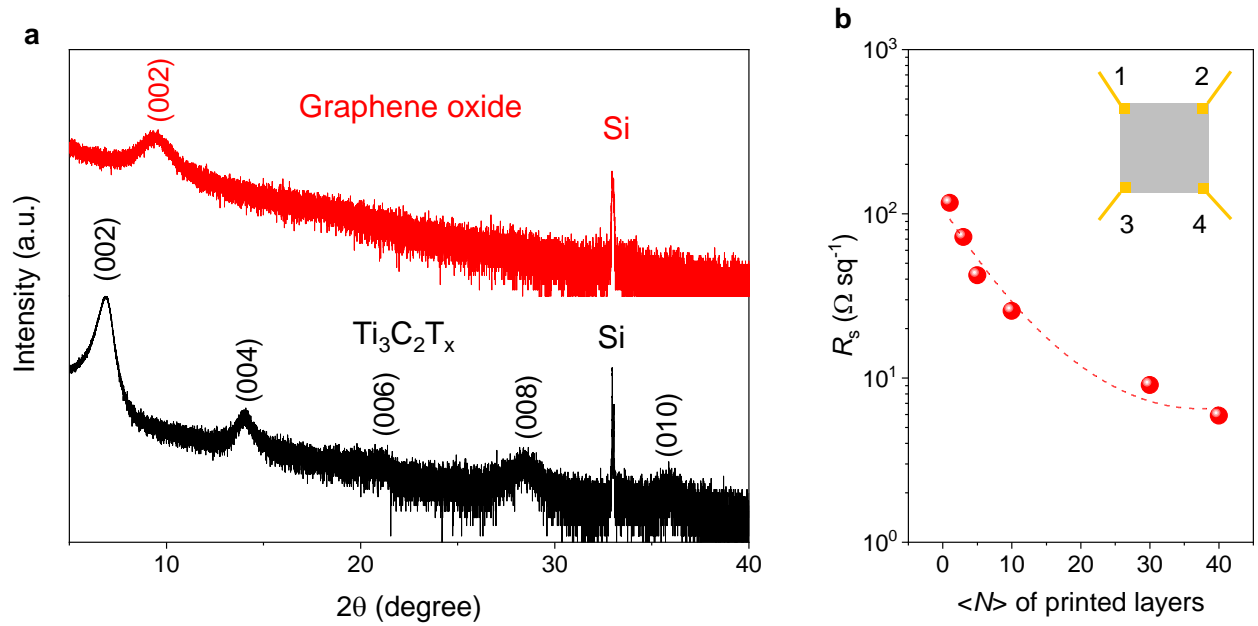

Figure 4.3 (a) XRD pattern of printed MXene and GO films on silicon substrates. (b) Sheet resistance $R_{\mathrm{s}}$ as function of the number of printed MXene layers $\left(5\right.$ by $5 \mathrm{~cm}^{2}$ ) on $\mathrm{Si} / \mathrm{SiO}_{2}$. The inset shows a schematic representation of the sheet resistivity as measured by the Van der Pauw method. The ink concentration was around $4.5 \mathrm{mg} \mathrm{mL}^{-1}$. 
Additive-free water-based MXene and GO inks were successfully prepared as shown in Figure 4.2a. Due to its high GO concentration, the GO ink has a dark brown color. The thickness of MXene and GO nanosheets were determined by atomic force microscopy (AFM) to be around $1.5 \mathrm{~nm}$ and $1 \mathrm{~nm}$, respectively, indicating a unilamellar structure for both types (Figure S4.1a,b, Appendices). The lateral sizes of MXene and GO nanosheets estimated from AFM images were about $0.76 \mu \mathrm{m}$ for MXene nanosheets and $0.78 \mu \mathrm{m}$ for GO nanosheets (Figure S4.1c, Appendices). To evaluate the ink printability, the inverse Ohnesorge number $Z$, which is defined as $Z=(\alpha \rho \gamma)^{1 / 2} / \eta$, was employed. Here, $\alpha$ is the nozzle diameter, $\rho$ is the density, $\gamma$ is the surface tension, and $\eta$ is the viscosity of the fluid. The surface tension and viscosity of MXene ink were $80.3 \mathrm{mN} \mathrm{m}^{-1}$ and $1.4 \mathrm{mPa}$, respectively. The nozzle diameter of $21.5 \mu \mathrm{m}$ and the value of surface tension, viscosity result in a $Z$ value of about 30 for MXene ink. The surface tension and viscosity of GO inks were $129.4 \mathrm{mN} \mathrm{m}^{-1}$ and $20.2 \mathrm{mPa}$ s, respectively, leading to a $Z$ value of about 3. Stroboscopic images of ink droplet formation versus time illustrated the quality of the inks (Figure S4.2, Appendices). No satellite droplets were observed for MXene and GO inks. Both inks showed good wetting on silicon substrates, as confirmed by AFM mappings, and the cross-sectional profile of the AFM images further confirmed the uniform deposition of both MXene and GO inks (Figure S4.3, Appendices). The wrinkles in printed GO droplets were caused by interactions between adjacent GO sheets $^{18}$. Figure $4.2 \mathrm{~b}$ shows two examples of printed patterns obtained by MXene ink on flexible polyimide substrate, demonstrating flexibility in pattern design and large area coating with multiple printing passes. The scanning electron microscopy (SEM) images of printed MXene (Figure 4.2c) and GO (Figure 4.2d) nanosheet films on $\mathrm{Si} / \mathrm{SiO}_{2}$ substrates show uniformity and continuity over large surface areas. It is worth noting that the sheets in both printed films showed a high degree of horizontal orientation and a layer-by-layer structure, which will facilitate the transport of electrolyte ions in in-plane structured devices such as MSCs.

As shown in Figure 4.3a, the XRD pattern of a printed MXene film shows strong ordering in the $c$ direction with a (002) peak at $6.8^{\circ}$, thus confirming the horizontal orientation of nanosheets in printed films. The smaller angle than in dry MXene films, where the same peak is at $8.9^{\circ}$ (Figure $\mathrm{S} 4.4 \mathrm{a}$, Appendices), indicates wider spacing between the layers in the printed film and intercalation of spatially confined $\mathrm{H}_{2} \mathrm{O}$ molecules ${ }^{19}$. The XRD pattern of a printed GO film shows a peak at $2 \theta=9.6^{\circ}$, which corresponds with a $d$ spacing of $0.92 \mathrm{~nm}$, suggesting that electrolyte ion transport is predominant in horizontal rather than in vertical direction. The sheet resistance $R_{\mathrm{S}}$ of printed MXene films could be tuned by the number of printed layers. As shown in Figure $4.3 \mathrm{~b}$, the $R_{\mathrm{S}}$ of MXene films on $\mathrm{Si} / \mathrm{SiO}_{2}$ substrates decreased rapidly from $116.7 \Omega \mathrm{sq}^{-1}$ (printed layers $\left.<N>=1\right)$ to around $5.9 \Omega \mathrm{sq}^{-1}(<N>=40)$ with an ink concentration of around $4.5 \mathrm{mg} \mathrm{ml}^{-1}$. 

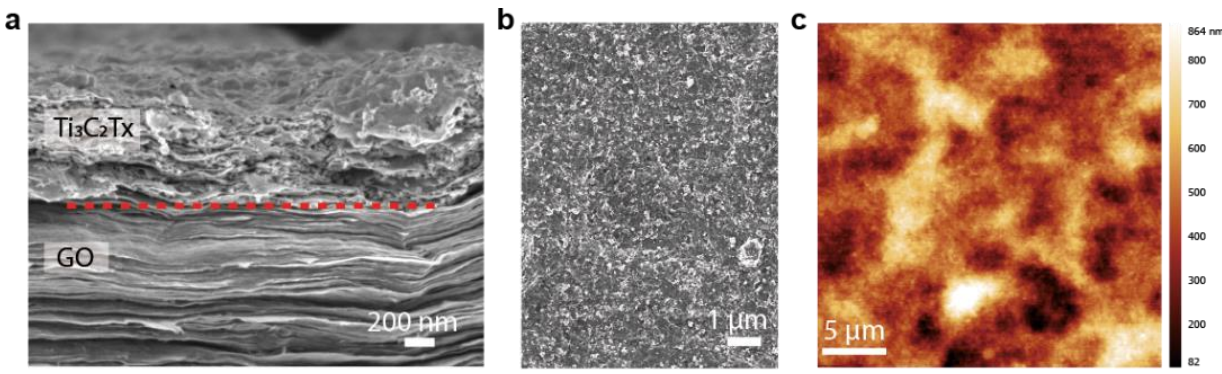

Figure 4.4 (a) Cross-sectional SEM image of inkjet-printed SSC. The dashed line roughly indicates the boundary between MXene (above) and GO (below) phases as a guide to the eye. (b) Top view SEM of top MXene electrode. (c) AFM topography of $30 \times 30 \mu \mathrm{m}^{2}$ scan area in device.

The solid-state symmetrical SSCs consist of a printed solid-state GO electrolyte sandwiched between two printed MXene electrodes (Figure 4.1, Figure S4.6a,c, Appendices). A clear boundary between electrolyte and electrodes can be identified, demonstrating well-defined spatial separation between the phases (Figure S4.6b, Appendices). To avoid remixing of nanosheets at the printed interfaces, printed nanosheet layers were solidified by heating at $50{ }^{\circ} \mathrm{C}$ for $1 \mathrm{~h}$ before printing another material on top. Cross-sectional SEM analysis indicated an intimate and stable contact between the MXene electrodes and the GO electrolyte (Figure 4.4a, Figure S4.6d-f, Appendices). The top-view SEM image of a MXene electrode shows continuous features of printed electrodes without cracks or pin holes, illustrating the high quality of printed films (Figure 4.4b). Due to the high resolution brought by inkjet printing, all printed 30L SSCs exhibited a low root mean square roughness (RMS) of $\approx 130 \pm 25 \mathrm{~nm}$ at a device thickness of around $4 \mu \mathrm{m}$ (Figure 4.4c). Element mappings from energydispersive x-ray spectroscopy (EDS) further confirm the stable and sharp interface between MXene electrodes and GO electrolyte (Figure S4.7, Appendices).

To investigate the electrochemical performances of all-inkjet-printed SSCs, MXene electrodes with thicknesses of 10 and 30 printed layers (from here on referred to as 10L SSC and 30L SSC, respectively) were fabricated. As shown in Figure 4.5a, 30L SSC shows a higher specific capacitance than 10L SSC from cyclic voltammetry (CV) at scan rate of 10 $\mathrm{mV} \mathrm{s}^{-1}$. The quasi-rectangular $\mathrm{CV}$ curves show the pseudo-capacitive behavior of the devices. More specifically, 30L SSC still exhibits a quasi-rectangular shape even at high scan rate (Figure S4.8a,c, Appendices). Galvanostatic charge/discharge (GCD) data of both devices at current densities ranging from $40-200 \mu \mathrm{A} \mathrm{cm}^{-2}$ (Figure S4.8b,d, Appendices) are shown in Figure $4.5 \mathrm{~b}$. The $30 \mathrm{~L} \mathrm{SSC}$ exhibits $C_{\mathrm{A}}$ as high as $9.8 \mathrm{mF} \mathrm{cm}^{-2}$ at current density of $40 \mu \mathrm{A} \mathrm{cm}^{-}$ ${ }^{2}$, while $10 \mathrm{~L} \mathrm{SSC}$ exhibits $C_{\mathrm{A}}$ of $3 \mathrm{mF} \mathrm{cm}^{-2}$ at same current density, indicating that $C_{\mathrm{A}}$ is roughly proportional with the number of printed MXene layers. 
a

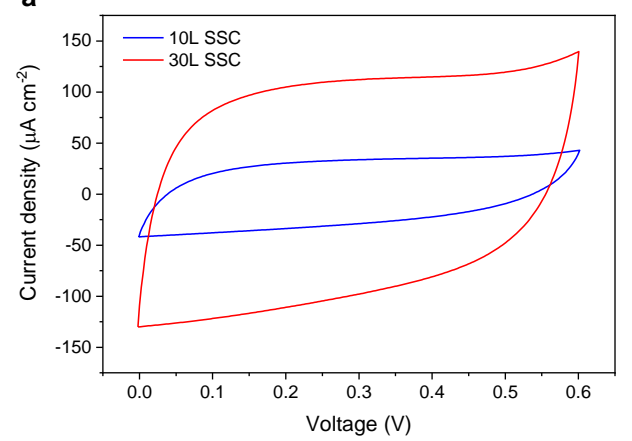

C

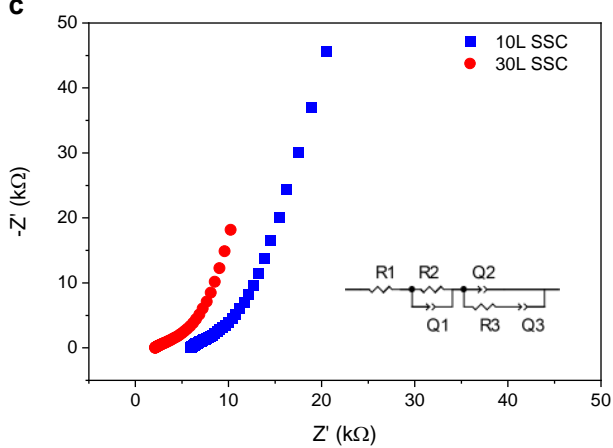

b

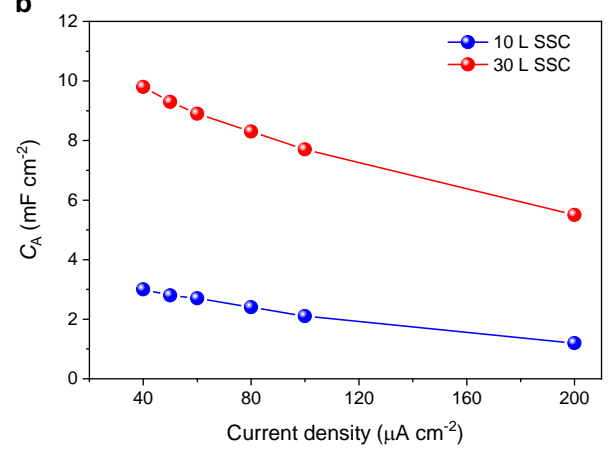

d

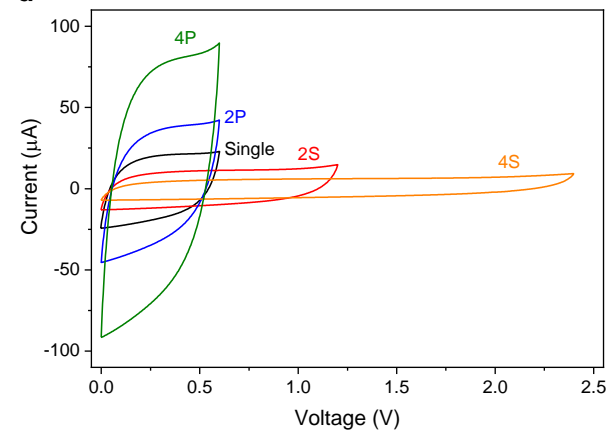

Figure 4.5 Electrochemical performance of all-inkjet-printed SSCs. (a) CV of as-made 10L SSC and 30L SSC at 10 $\mathrm{mV} \mathrm{s}^{-1}$. (b) $C_{\mathrm{A}}$ of as-made 10L SSC and 30L SSC at different current densities. (c) Nyquist plots of as-made 10L SSC and 30L SSC. The inset shows the equivalent circuit to which the experimental data were fitted. (d) CV diagram of 1, 2 and 4 supercapacitors connected in series and in parallel.

Both types of devices were characterized without liquid electrolyte. The mobile electrolyte ions needed for charging/discharging these devices are therefore thought to arise from the hydrolysis of functional oxygen-bearing groups on the solid GO electrolyte ${ }^{11}$. Free water molecules present between GO sheets may facilitate proton transport via the Grotthuss mechanism ${ }^{20}$ or by diffusion of hydronium ions within the interlayer spaces ${ }^{11}$. Intimate contact between the GO electrolyte and MXene electrodes will facilitate proton transfer between different SSC components. Proton movement inside the MXene electrodes probably proceeds via confined water molecules that are trapped between MXene sheets.

Electrochemical impedance spectroscopy (EIS) was conducted on both devices in the frequency range from $10 \mathrm{mHz}$ to $10 \mathrm{kHz}$. The experimental data were fitted to the equivalent circuit shown in the inset of Figure 4.5c. It consists of a constant phase element (CPE) $\mathrm{Q}_{2}$ that represents the surface capacitance of the device, in parallel with a CPE $\mathrm{Q}_{3}$ that represents the slower diffusion-controlled volume capacitance. The electrolyte is represented by the $\mathrm{R}_{2}\left(\mathrm{Q}_{1}\right)$ sub-circuit, and the electrode resistance by $\mathrm{R}_{1}$. Sample $10 \mathrm{~L}$ SSC has a larger $\mathrm{R}_{1}$ than 
30L SSC, showing that thicker electrodes exhibit a lower resistance (Table S1; $5.9 \mathrm{k} \Omega$ versus $2.1 \mathrm{k} \Omega$ ). The impedance in the low frequency range suggests mixed surface absorption and diffusional control of the devices, which are the double layer capacitance $\left(\mathrm{Q}_{2}\right)$ and charge transfer diffusion impedance $\mathrm{Q}_{3}$. The 30L SSC device has a smaller charge transfer resistance $\left(\mathrm{R}_{3}\right)$ in the lower frequency range than $10 \mathrm{~L} \mathrm{SSC}(1.9 \mathrm{k} \Omega$ versus $12.9 \mathrm{k} \Omega)$. To demonstrate the potential for practical applications at high voltages, the as-made $30 \mathrm{~L} \mathrm{SSCs}$ were connected in series and in parallel configurations. As shown in Figure 4.5d, the voltage window reached $1.2 \mathrm{~V}$ and $2.4 \mathrm{~V}$ with two and four devices connected in series, respectively. The current was increased by a factor of $\sim 2$ and 4 with two and four devices connected in parallel, respectively.

The electrochemical performance of SSCs could be enhanced by addition of liquid electrolytes. As shown in Figure 4.6a, excess deionized (DI) water resulted in a higher $C_{\mathrm{A}}$ of $12.1 \mathrm{mF} \mathrm{cm}^{-2}$ than in the as-made device, probably due to enhanced ion transport in liquid media. Aqueous electrolytes such as $5 \mathrm{M} \mathrm{LiCl}$ and $0.5 \mathrm{M} \mathrm{Na}_{2} \mathrm{SO}_{4}$ introduce additional electrolyte ions that enhance ionic transport at the same time, increasing the $C_{\mathrm{A}}$ further to 13.6-14.1 $\mathrm{mF} \mathrm{cm}^{-2}$ (Figure S4.9, Appendices). The as-made 30L SSC exhibited an areal energy density $\left(E_{\mathrm{A}}\right)$ of $0.49 \mu \mathrm{Wh} \mathrm{cm}{ }^{-2}$ at a power density $\left(P_{\mathrm{A}}\right)$ of $12.55 \mu \mathrm{W} \mathrm{cm}{ }^{-2}$, while $E_{\mathrm{A}}$ increased to $0.71 \mu \mathrm{Wh} \mathrm{cm}^{-2}$ at $P_{\mathrm{A}}$ of $12.48 \mu \mathrm{W} \mathrm{cm} \mathrm{cm}^{-2}$ by adding a drop of $5 \mathrm{M} \mathrm{LiCl} \mathrm{electrolyte}$ into the as-made device (Figure 4.6b). Apparently, the presence of liquid electrolyte has a positive effect on the energy density, however the precise mechanism remains unclear. The $E_{\mathrm{A}}$ of as-made $30 \mathrm{~L} \mathrm{SSC}$ is higher than the recently reported extrusion-printed MXene MSC with $\mathrm{H}_{2} \mathrm{SO}_{4}$-poly(vinyl alcohol, PVA) gel electrolyte $\left(E_{\mathrm{A}}\right.$ of $0.32 \mu \mathrm{Wh} \mathrm{cm}^{-2}$ at $P_{\mathrm{A}}$ of $11.40 \mu \mathrm{W}$ $\left.\mathrm{cm}^{-2}\right)^{21}$, fully printed MSC with GO electrolyte ${ }^{22}$, all printed MSC with graphene electrodes and printed polyelectrolyte ${ }^{23}$, printed graphene $\mathrm{MSC}^{24}$, and $\mathrm{MXene} /$ single-walled carbon nanotube supercapacitor ${ }^{25}$. The $30 \mathrm{~L}$ SSC exhibits good cycling stability with a capacitance retention of $\sim 100 \%$ after 10000 cycles (Figure S4.10a, Appendices). Moreover, the allprinted SSC shows high mechanical stability with a bending radius of about $1 \mathrm{~cm}$, as shown in Figure S4.10b (Appendices). 

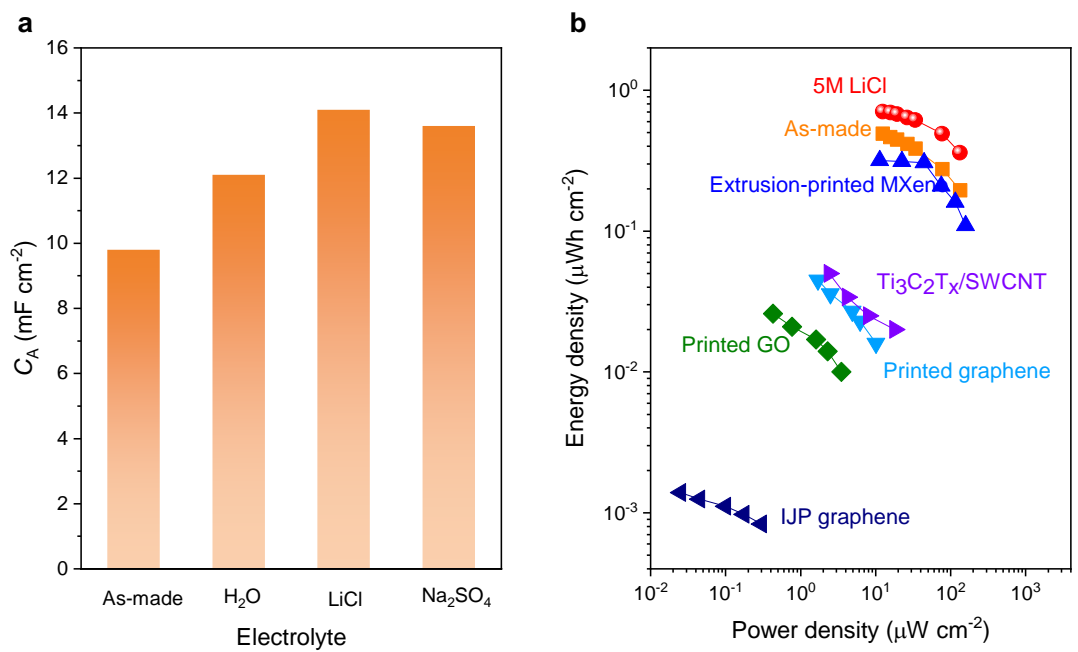

Figure 4.6 (a) $C_{\mathrm{A}}$ of $30 \mathrm{~L} \mathrm{SSC}$ with different electrolytes (DI water, $5 \mathrm{M} \mathrm{LiCl}$ and $0.5 \mathrm{M} \mathrm{Na}_{2} \mathrm{SO}_{4}$ in water, respectively) on top of the devices. (b) Ragone plot of all-inkjet-printed SSC with other different systems.
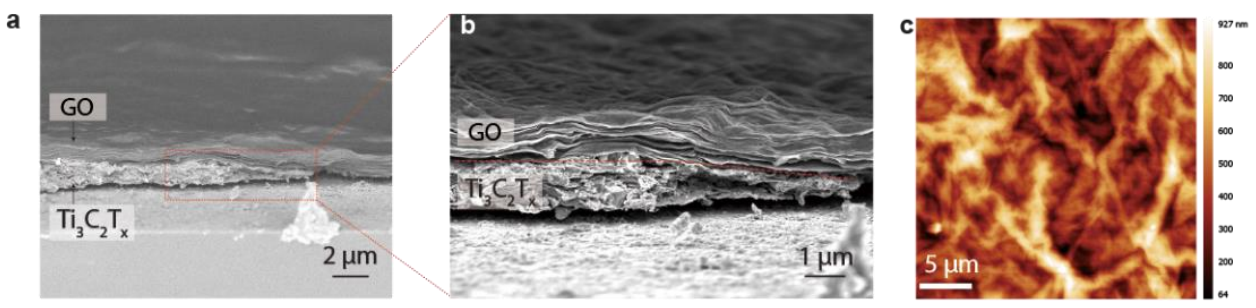

Figure 4.7 (a, b) Cross-sectional SEM images of all-inkjet-printed MSC. The dashed line roughly divides MXene electrode (below) and GO electrolyte (above). (c) AFM topography of top GO electrolyte in (b).

MSCs were fabricated by printing MXene nanosheets with interdigitated structure as electrodes on polyimide substrate, followed by a printed layer of GO nanosheets on top of/over the MXene electrodes to serve as solid-state electrolyte (Figure 4.1, bottom). MXene electrodes with 10, 20 and 30 printed layers (refers to as 10L MSC, 20L MSC and 30L MSC, respectively) were printed (Figure S4.11, Appendices). The printed MXene electrodes show sharp features at the edges, indicating the stability of the MXene ink and the high reliability of the inkjet printing process, and the sharp edges were still retained after printing GO nanosheets on top. EDS elemental mapping confirms the presence of a well-defined and stable top interface between MXene electrode and GO electrolyte (Figure S4.12, Appendices). The all-inkjet-printed 30L MSCs exhibited a low RMS of $\approx 157 \pm 20 \mathrm{~nm}$ at a device thickness of around $2.3 \mu \mathrm{m}$ (Figure 4.7c). However, the contact at the cross-section between MXene 
electrode and GO electrolyte is poor (Figure 4.7a,b), which is likely the cause of low current response in the CV measurement (Figure 4.8a). Most likely, only GO sheets near the heterostructure interface contribute to the capacitance, because the protons are generated via hydrolysis of functional groups on GO.

Electrochemical measurements were performed on as-made all-solid-state MSCs and on MSCs to which excess aqueous electrolyte had been added. The electrochemical performance of 30L MSC improved considerably upon addition of water, due to enhanced proton mobility (Figure 4.8a). The CV curves of MSCs with varying electrode thicknesses demonstrate that thicker electrodes with more active surface sites show higher $C_{\mathrm{A}}$ (Figure $4.8 \mathrm{~b}$, Figure $\mathrm{S} 4.13 \mathrm{a}-$ c, Appendices). GCD further confirms that 30L MSC exhibits a higher capacitance than the other two devices (Figure 4.8c, Figure S4.13d-f, Appendices). The $C_{\mathrm{A}}$ of 30L MSC reached to $3.1 \mathrm{mF} \mathrm{cm}^{-2}$, while 10 and 20 layer devices reached 1.2 and $1.9 \mathrm{mF} \mathrm{cm}^{-2}$ at a current density of $20 \mu \mathrm{A} \mathrm{cm}^{-2}$, respectively (Figure $4.8 \mathrm{~d}$ ).
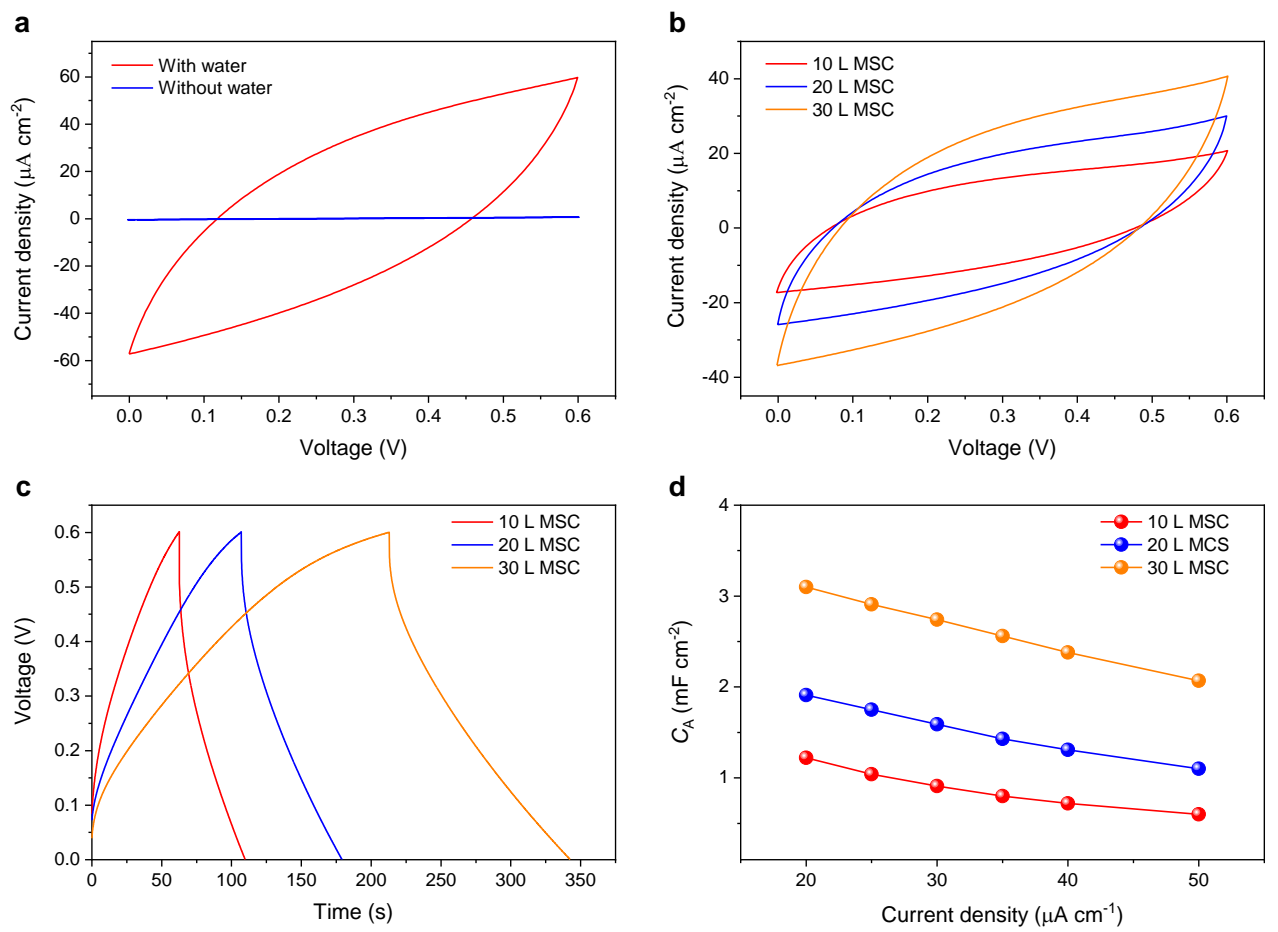

Figure 4.8 Electrochemical performance of all-inkjet-printed MSCs. (a) CV of 30L MSC with and without added water on top of the device at $20 \mathrm{mV} \mathrm{s}^{-1}$. (b) CV of printed $10 \mathrm{~L} / 20 \mathrm{~L} / 30 \mathrm{~L} \mathrm{MSCs}$ at $10 \mathrm{mV} \mathrm{s}^{-1}$. (c) GCD of printed $10 \mathrm{~L}$ and 30L MSCs at $15 \mu \mathrm{A} \mathrm{cm}^{-2}$. (d) $C_{\mathrm{A}}$ of printed $10 \mathrm{~L} / 20 \mathrm{~L} / 30 \mathrm{~L}$ MSCs with different current densities. 

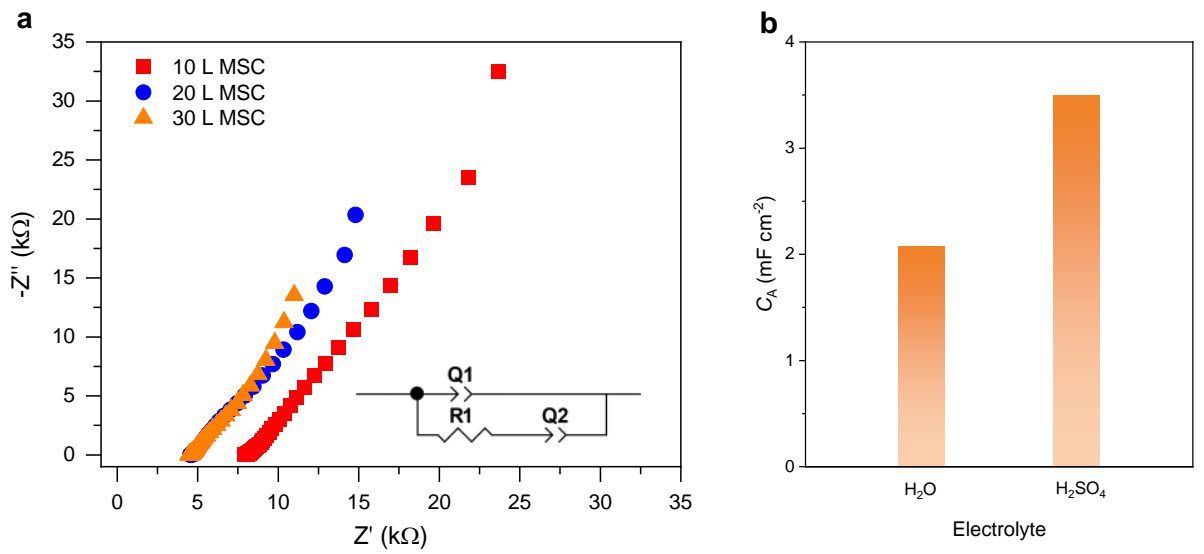

Figure 4.9 (a) Nyquist plots of printed 10L/20L/30L MSCs. The inset is the fitted equivalent circuit for the low frequency range of the impedance response. (b) $C_{\mathrm{A}}$ of $30 \mathrm{~L} \mathrm{MSC}$ with excess $\mathrm{H}_{2} \mathrm{O}$ and $0.5 \mathrm{M} \mathrm{H}_{2} \mathrm{SO}_{4}$ at current density of $50 \mu \mathrm{A} \mathrm{cm}^{-2}$.

EIS suggests that the charge transfer resistance $\left(\mathrm{R}_{1}\right)$ of the 30 layers thick MXene electrode device is lower than the other two devices $(4.1 \mathrm{k} \Omega, 11.9 \mathrm{k} \Omega$ and $12.0 \mathrm{k} \Omega$ for $10 \mathrm{~L} \mathrm{SSC}$, 20LSSC and 30L SSC, respectively; Figure 4.9a, Table S2). Similar to the SSC, the equivalent circuits in the low frequency range suggests mixed surface absorption and diffusional control, i.e. the double layer capacitance $\left(\mathrm{Q}_{1}\right)$ and the charge transfer diffusion impedance $\left(\mathrm{Q}_{2}\right)$. Addition of a $0.5 \mathrm{M} \mathrm{H}_{2} \mathrm{SO}_{4}$ electrolyte solution onto $30 \mathrm{~L} \mathrm{MSC}$ resulted in a a higher capacitance than in devices with excess water (Figure 4.9b, Figure S4.14, Appendices). The $\mathrm{H}_{2} \mathrm{SO}_{4}$ electrolyte provides additional protons that enhance the ionic conductivity, leading to lower series resistances.

\subsection{Conclusion}

In conclusion, we demonstrated all-inkjet-printed solid-state supercapacitors based on 2D MXene/GO/MXene. Due to the high ionic conductivity of GO films, the printed SSC without liquid electrolyte showed an $E_{\mathrm{A}}$ of $0.49 \mu \mathrm{Wh} \mathrm{cm}^{-2}$ at a $P_{\mathrm{A}}$ of $12.55 \mu \mathrm{W} \mathrm{cm}{ }^{-2}$. The $C_{\mathrm{A}}$ can be increased further by adding liquid electrolyte. Further optimization on materials, printed electrodes thickness and electrodes configuration will enhance device performance further. Printed supercapacitors show high potential for use in small power source units for flexible electronics.

\subsection{References}

1. Novoselov, K. S., Mishchenko, A., Carvalho, A. \& Castro Neto, A. H. 2D materials 
and van der Waals heterostructures. Science 353, aac9439 (2016).

2 Geim, A. K. \& Grigorieva, I. V. Van der Waals heterostructures. Nature 499, 419425 (2013).

3 Liu, Y. et al. Van der Waals heterostructures and devices. Nat. Rev. Mater. 1, 16042 (2016).

4. Anasori, B., Lukatskaya, M. R. \& Gogotsi, Y. 2D metal carbides and nitrides (MXenes) for energy storage. Nat. Rev. Mater. 2, 16098 (2017).

5. Naguib, M., Mochalin, V. N., Barsoum, M. W. \& Gogotsi, Y. 25th anniversary article: MXenes: a new family of two-dimensional materials. Adv. Mater. 26, 992-1005 (2014).

6. Zhang, Y. Z. et al. MXenes stretch hydrogel sensor performance to new limits. Sci. Adv. 4, eaat0098 (2018).

7. Agresti, A. et al. Titanium-carbide MXenes for work function and interface engineering in perovskite solar cells. Nat. Mater. 18, 1228-1234 (2019).

8. Ghidiu, M., Lukatskaya, M. R., Zhao, M. Q., Gogotsi, Y. \& Barsoum, M. W. Conductive two-dimensional titanium carbide 'clay' with high volumetric capacitance. Nature 516, 78-U171 (2014).

9. Xia, Y. et al. Thickness-independent capacitance of vertically aligned liquidcrystalline MXenes. Nature 557, 409-412 (2018).

10. Pomerantseva, E. \& Gogotsi, Y. Two-dimensional heterostructures for energy storage. Nat. Energy 2, 17089 (2017).

11. Gao, W. et al. Direct laser writing of micro-supercapacitors on hydrated graphite oxide films. Nat. Nanotechnol. 6, 496-500 (2011).

12. McManus, D. et al. Water-based and biocompatible 2D crystal inks for all-inkjetprinted heterostructures. Nat. Nanotechnol. 12, 343-350 (2017).

13. Kelly, A. G. et al. All-printed thin-film transistors from networks of liquidexfoliated nanosheets. Science 356, 69-73 (2017).

14. Worsley, R. et al. All-2D material inkjet-printed capacitors: toward fully printed integrated circuits. ACS Nano 13, 54-60 (2019).

15. Zhang, H. Ultrathin two-dimensional nanomaterials. ACS Nano 9, 9451-9469 (2015). 
16. Cain, J. D. et al. Sculpting liquids with two-dimensional materials: the assembly of $\mathrm{Ti}_{3} \mathrm{C}_{2} \mathrm{~T}_{\mathrm{x}}$ MXene sheets at liquid-liquid interfaces. ACS Nano 13, 12385-12392 (2019).

17. Hummers, W. S. \& Offeman, R. E. Preparation of graphitic oxide. J. Am. Chem. Soc. 80, 1339-1339 (1958).

18. Shen, X., Lin, X. Y., Yousefi, N., Jia, J. J. \& Kim, J. K. Wrinkling in graphene sheets and graphene oxide papers. Carbon 66, 84-92 (2014).

19. Mu, X. P. et al. Revealing the pseudo-intercalation charge storage mechanism of MXenes in acidic electrolyte. Adv. Funct. Mater. 29, 1902953 (2019).

20. Agmon, N. The Grotthuss mechanism. Chem. Phys. Lett. 244, 456-462 (1995).

21. Zhang, C. J. et al. Additive-free MXene inks and direct printing of microsupercapacitors. Nat. Commun. 10, 1795 (2019).

22. Sollami Delekta, S. et al. Fully inkjet printed ultrathin microsupercapacitors based on graphene electrodes and a nano-graphene oxide electrolyte. Nanoscale 11, 10172-10177 (2019).

23. Li, J. et al. Scalable fabrication and integration of graphene microsupercapacitors through full inkjet printing. ACS Nano 11, 8249-8256 (2017).

24. Sollami Delekta, S., Smith, A. D., Li, J. \& Ostling, M. Inkjet printed highly transparent and flexible graphene micro-supercapacitors. Nanoscale 9, 6998-7005 (2017).

25. Zhang, C. J. et al. Transparent, flexible, and conductive 2D titanium carbide (MXene) films with high volumetric capacitance. Adv. Mater. 29, 1702678 (2017). 


\section{APPENDICES}
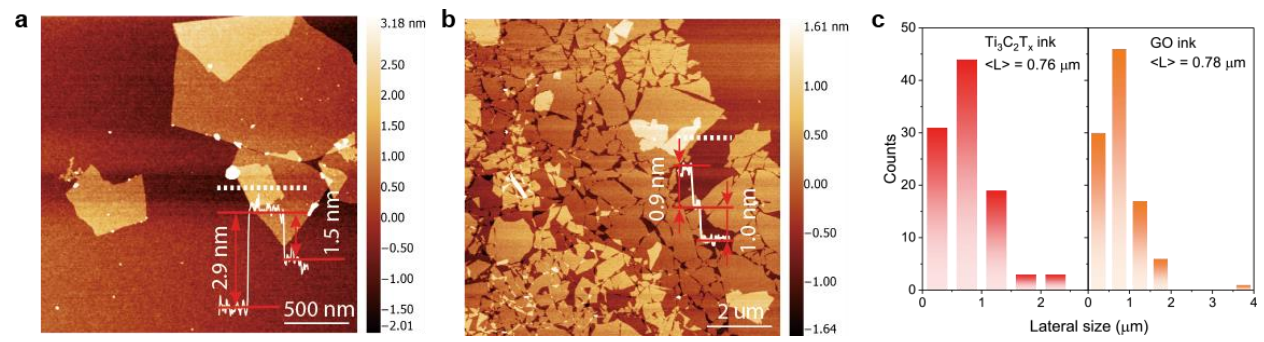

Figure S4.1 MXene and GO sheet thicknesses and lateral sizes. AFM images of (a) $\mathrm{Ti}_{3} \mathrm{C}_{2} \mathrm{~T}_{\mathrm{x}}$ and (b) GO nanosheets. (c) Lateral size distribution of $\mathrm{Ti}_{3} \mathrm{C}_{2} \mathrm{~T}_{\mathrm{x}}$ and $\mathrm{GO}$ nanosheets estimated by measuring 100 flakes dropcast on $\mathrm{Si} / \mathrm{SiO}$.
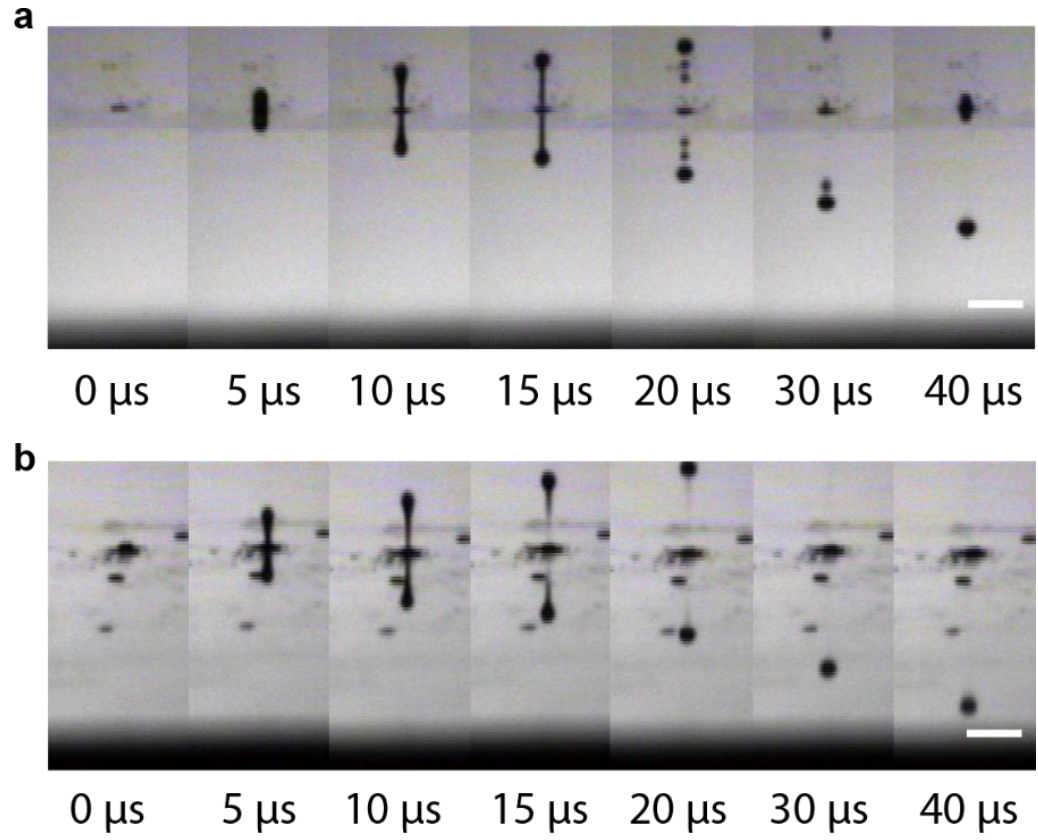

Figure S4.2 Ink stability. Stroboscopic images of (a) $\mathrm{Ti}_{3} \mathrm{C}_{2} \mathrm{~T}_{\mathrm{x}}$ and (b) GO ink droplet formation vs. time as observed from the printer camera. The scale bars in (a) and (b) are $50 \mu \mathrm{m}$.

To achieve a high quality inkjet printing process, the preparation of printable and stable inks is very important. Water-based MXene ink without any additives showed highly stable printing behavior during jetting, which may be attributed to the presence of functional groups like $-\mathrm{O},-\mathrm{OH}$ and $-\mathrm{F}$ on the surface of MXene sheets (Figure S4.2a) that help dispersion in water. To prepare a printable GO ink, Triton X-100 was added to the water-based graphene 
oxide ink in order to optimize the ink surface tension. As shown in Figure S4.2b, no satellite droplets were generated during jetting, indicating a printable and stable GO ink.

a

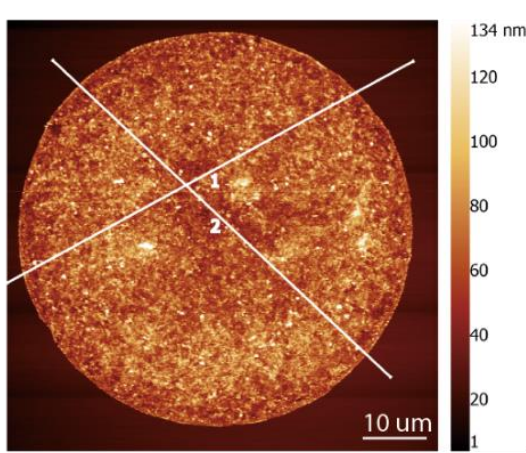

c

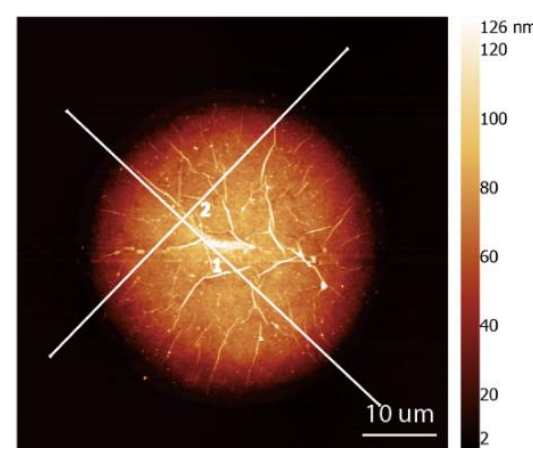

b

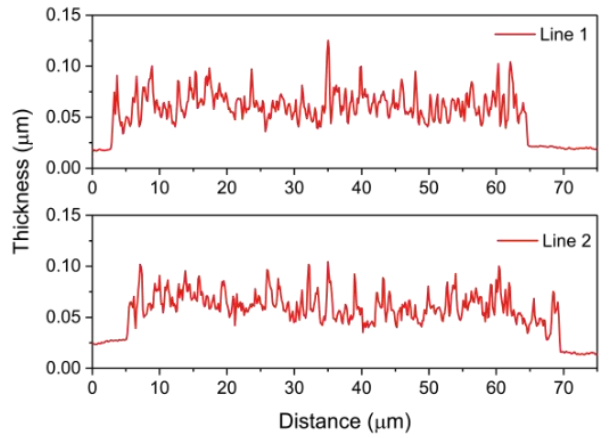

d

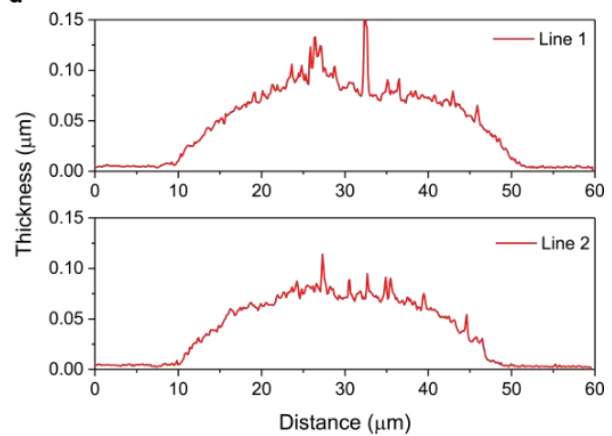

Figure S4.3 AFM analysis of inkjet printed MXene and GO single droplets. (a) AFM map of single printed $\mathrm{Ti}_{3} \mathrm{C}_{2} \mathrm{~T}_{x}$ droplet on silicon substrate. (b) Cross-sectional profiles along two different directions in (a). (c) AFM map of single printed GO droplet on silicon substrate. (d) Cross-sectional profiles along two different directions in (c). 
a

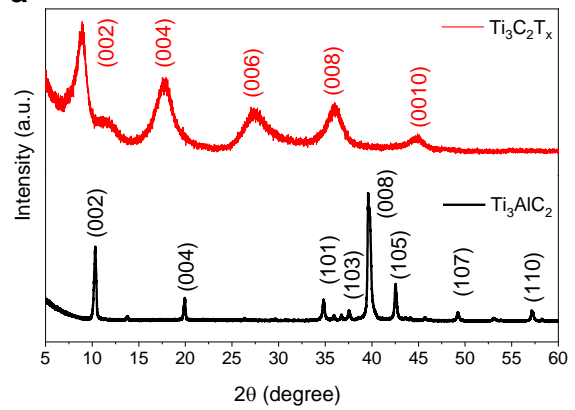

C

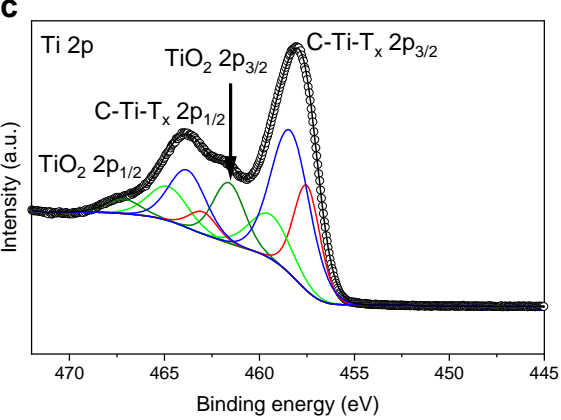

b

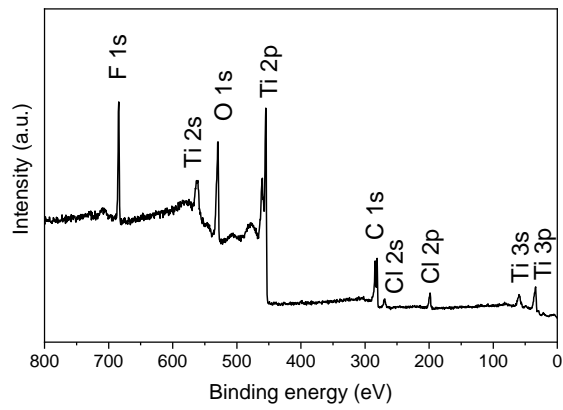

d

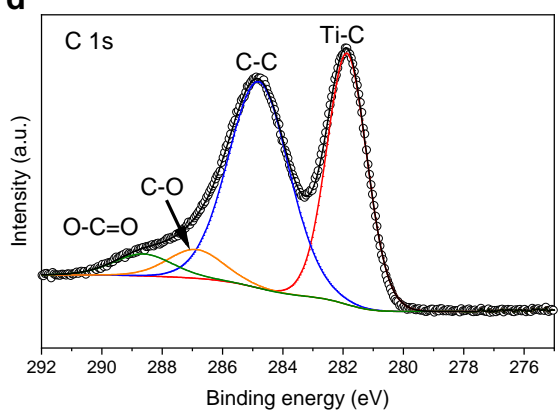

Figure S4.4 Characterization of $\mathrm{Ti}_{3} \mathrm{C}_{2} \mathrm{~T}_{\mathrm{x}}$ nanosheets. (a) XRD diagram of $\mathrm{Ti}_{3} \mathrm{AlC}_{2}$ powder and $\mathrm{Ti}_{3} \mathrm{C}_{2} \mathrm{~T}_{\mathrm{x}}$ nanosheets. (b) XPS survey spectrum of $\mathrm{Ti}_{3} \mathrm{C}_{2} \mathrm{~T}_{\mathrm{x}}$ nanosheets. High resolution XPS spectrum of (c) Ti $2 p$ and (d) $\mathrm{C} 1 s$ of $\mathrm{Ti}_{3} \mathrm{C}_{2} \mathrm{~T}_{\mathrm{x}}$ nanosheets.

As shown in Figure $\mathrm{S} 4.4 \mathrm{a}, \mathrm{Ti}_{3} \mathrm{C}_{2} \mathrm{~T}_{\mathrm{x}}$ nanosheets show larger interlayer spacings than $\mathrm{Ti}_{3} \mathrm{AlC}_{2}$ due to the successful removal of $\mathrm{Al}$, as can be concluded from the smaller diffraction angle of the (002) peak of the former. No Al peak was identified in the XPS survey spectrum, which further confirms the successful exfoliation of $\mathrm{Ti}_{3} \mathrm{C}_{2} \mathrm{~T}_{x}$ nanosheets (Figure S4.4b). The highresolution XPS spectrum of Ti $2 p$ could be fitted with four doublets (Ti $2 p_{3 / 2}-\mathrm{Ti} 2 p_{1 / 2}$ ). The Ti $2 p_{3 / 2}$ components located at $457.5,458.4$ and $459.4 \mathrm{eV}$ correspond to Ti-C, Ti (II) and Ti (III), respectively (Figure S4.4c). The peak at $461.6 \mathrm{eV}$ belongs to $\mathrm{Ti} 2 p_{3 / 2}$ of $\mathrm{TiO}_{2}$. The $\mathrm{C} 1 s$ is fitted by four components corresponding to Ti-C at a binding energy (BE) of $281.9 \mathrm{eV}, \mathrm{C}$ $\mathrm{C}$ at $284.8 \mathrm{eV}, \mathrm{C}-\mathrm{O}$ at $286.9 \mathrm{eV}$ and $\mathrm{O}-\mathrm{C}=\mathrm{O}$ at $288.6 \mathrm{eV}$ (Figure $\mathrm{S} 4.4 \mathrm{~d}$ ). The Ti-C peak is from MXene, while the other three peaks are from MXene surface termination group originated from chemical etching. The high resolution XPS spectra of Ti $2 p$ and C $1 s$ reveal that the MXene surfaces are terminated by $\mathrm{F}, \mathrm{O}$ and $\mathrm{OH}$ functional groups. 

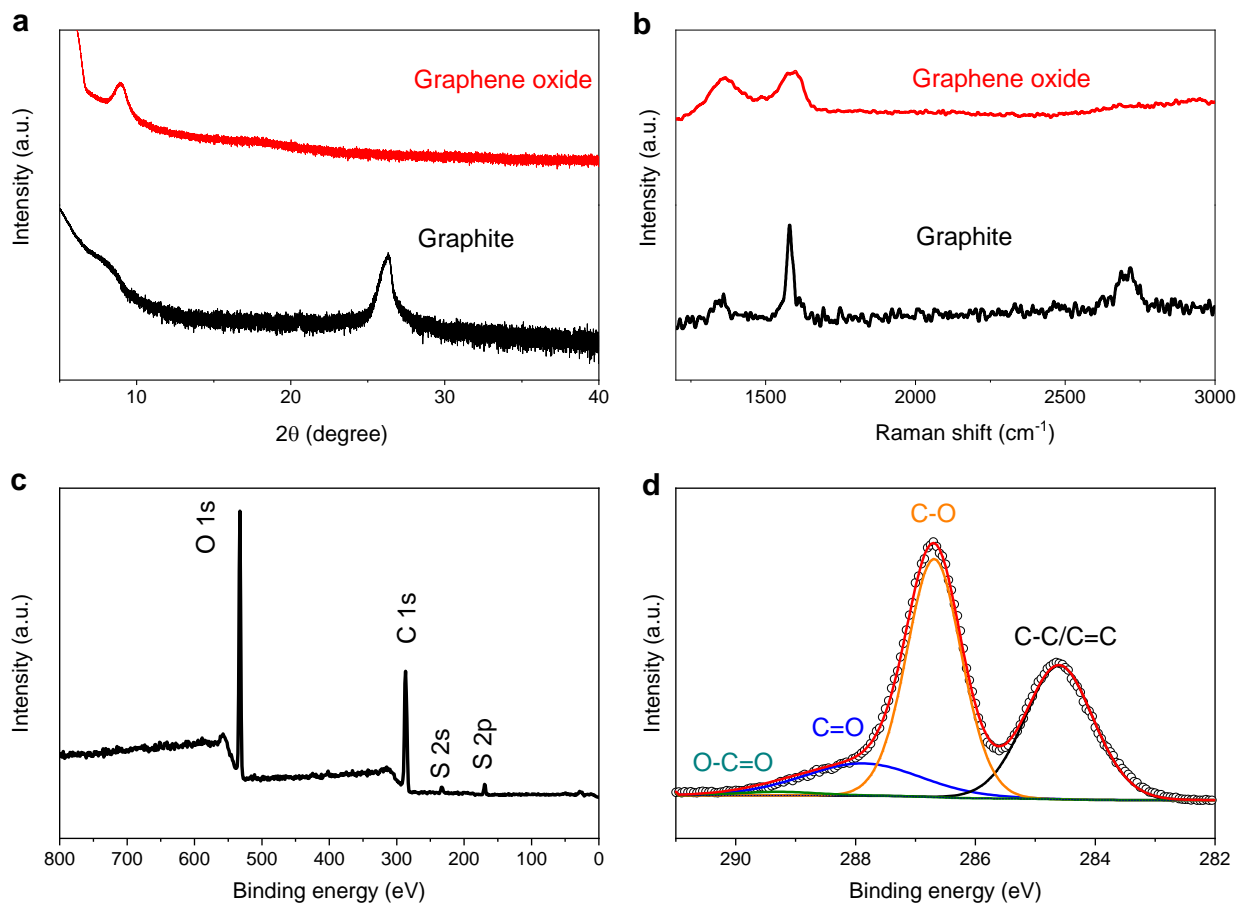

Figure S4.5 Characterization of GO nanosheets. (a) XRD of graphite and graphene oxide. (b) Raman spectrum of graphite and graphene oxide. (c) XPS spectrum of graphene oxide. (d) High resolution XPS spectrum of C $1 s$.

The XRD pattern of pristine graphite shows a (002) peak with an interlayer spacing of $3.4 \AA$, while the corresponding (002) peak in graphene oxide shows an interlayer spacing of $9.7 \AA$. This indicates successful exfoliation of graphite to graphene oxide nanosheets (Figure S4.5a). The Raman spectrum of pristine graphite shows a strong and sharp $G$ peak at $1581 \mathrm{~cm}^{-1}$ that is attributed to the first-order scattering of the $\mathrm{E}_{2 \mathrm{~g}}$ mode. This $\mathrm{G}$ peak becomes broader and shifts to $1599 \mathrm{~cm}^{-1}$ in graphene oxide. Furthermore, the appearance of a D band at $1365 \mathrm{~cm}^{-}$ ${ }^{1}$ indicates the reduction in size of the in-plane $s p^{2}$ domains (Figure S4.5b). The XPS survey spectra reveal that the main peaks are $\mathrm{C}$ and $\mathrm{O}$, the small peaks at a $\mathrm{BE}$ of $169.9 \mathrm{eV}$ and 233 $\mathrm{eV}$ come from residual S (Figure S4.5c). The high-resolution $\mathrm{C} 1 s$ spectrum demonstrates the high oxidation degree of graphene oxide with four components corresponding to $\mathrm{C}$ $\mathrm{C} / \mathrm{C}=\mathrm{C}$ at a $\mathrm{BE}$ of $284.8 \mathrm{eV}, \mathrm{C}-\mathrm{O}$ at $286.9 \mathrm{eV}, \mathrm{C}=\mathrm{O}$ at $288.1 \mathrm{eV}$ and $\mathrm{O}-\mathrm{C}=\mathrm{O}$ at $289.5 \mathrm{eV}$ (Figure $\mathrm{S} 4.5 \mathrm{~d}$ ). The $\mathrm{C} 1 s$ XPS spectrum of $\mathrm{GO}$ confirms a $\mathrm{C} / \mathrm{O}$ ratio of about 2.1 for $\mathrm{GO}$ nanosheets, indicating a high concentration of functional groups that can hydrolyze to generate protons for charging and discharging. 

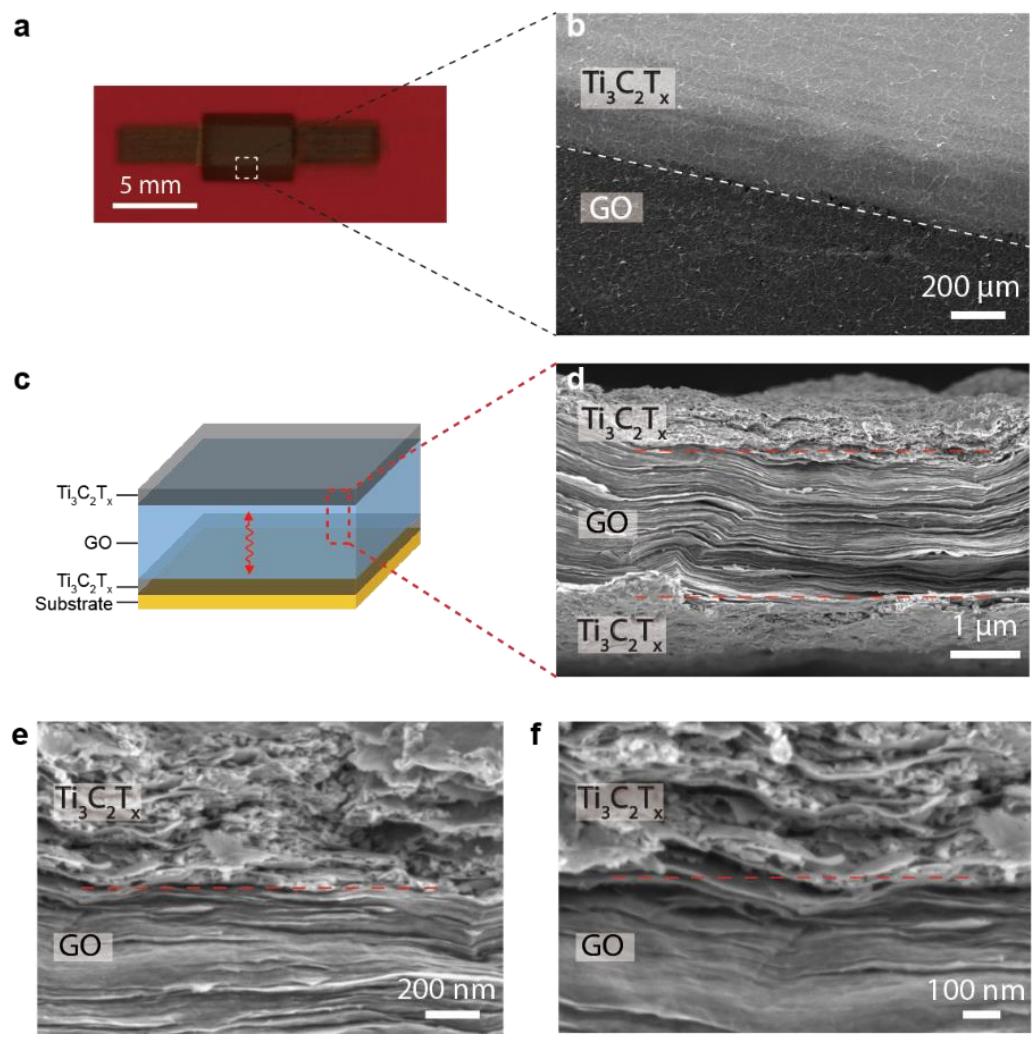

Figure S4.6 30L SSC device morphology. (a) Optical image of all-inkjet-printed 30L SSCs on flexible polyimide substrate. (b) SEM image of the boundary between $\mathrm{Ti}_{3} \mathrm{C}_{2} \mathrm{~T}_{x}$ electrode and GO electrolyte. (c) Schematic representation of sandwiched supercapacitor from networks of nanosheets. (d-f) Cross-sectional SEM images of allinkjet-printed SSC interfaces. The dashed lines roughly divide $\mathrm{Ti}_{3} \mathrm{C}_{2} \mathrm{~T}_{x}$ and $\mathrm{GO}$ and serve as a guide to the eye only.

Achieving well controlled and sharp interfaces is a significant challenge for printed heterostructures. The interface plays an important role in device performance. Adding binders such as xanthan gum into inks has been demonstrated to control the structure of the inkjet printed heterostructure interface. However, the performance could also be affected by the presence of (organic) binders. Annealing printed heterostructures at high temperatures can lead to removal of the binder, but it limits the choice of substrates to thermally stable ones. Here, we successfully inkjet printed vertical heterostructures without any sign of redispersion at the interface by drying the printed patterns before printing the next layer with different nanosheets. Drying was performed at $50{ }^{\circ} \mathrm{C}$, which is a relatively low temperature and is applicable to most substrates including paper and polymer substrates (Figure S4.6a-b). The cross-sectional SEM images a sharp interface with good contact between two different nanosheets (Figure S4.6c-f). 

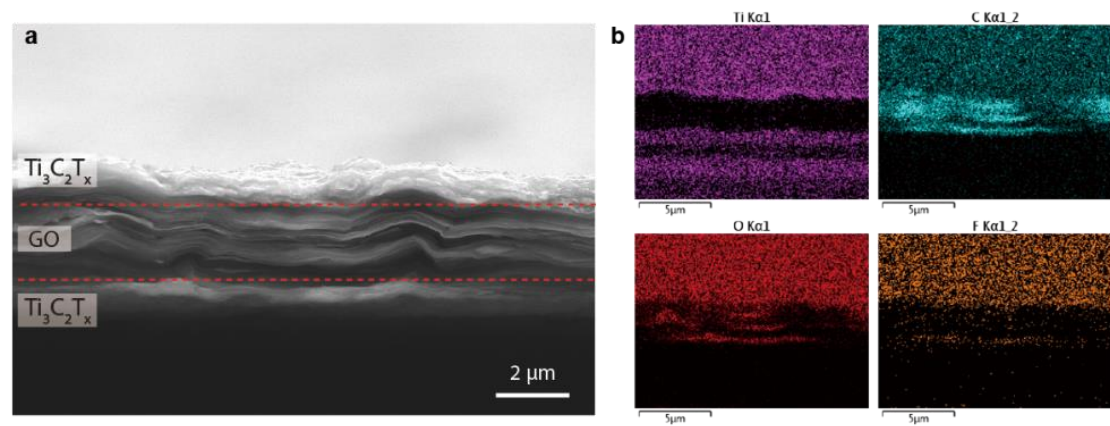

Figure S4.7 (a) Cross-sectional SEM of all-inkjet-printed SSC and (b) EDS elemental mapping of the elements Ti, $\mathrm{C}, \mathrm{O}$ and $\mathrm{F}$.
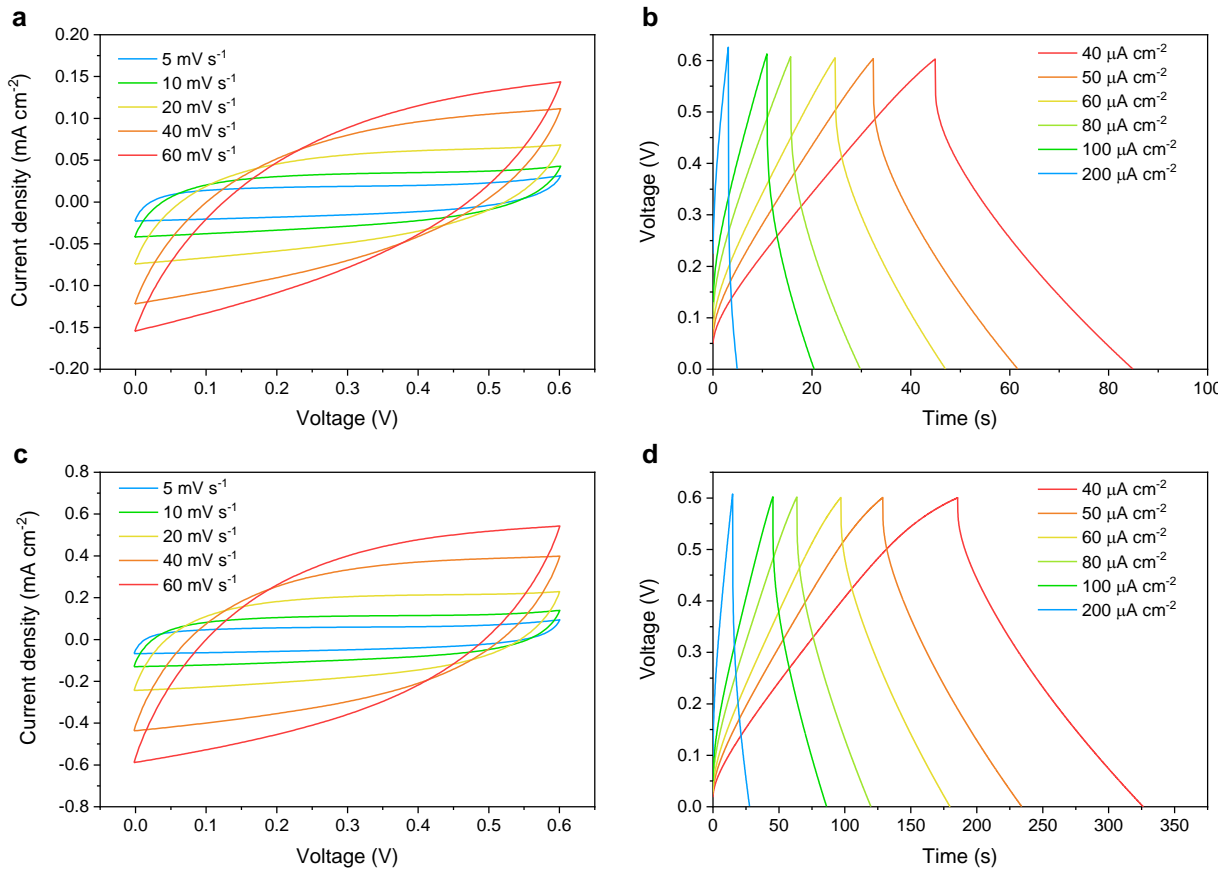

Figure S4.8 CV curves of (a) 10L SSC and (c) 30L SSC as-made devices. GCD curves of (b) 10L SSC and (d) 30L SSC as-made devices at different current densities. 

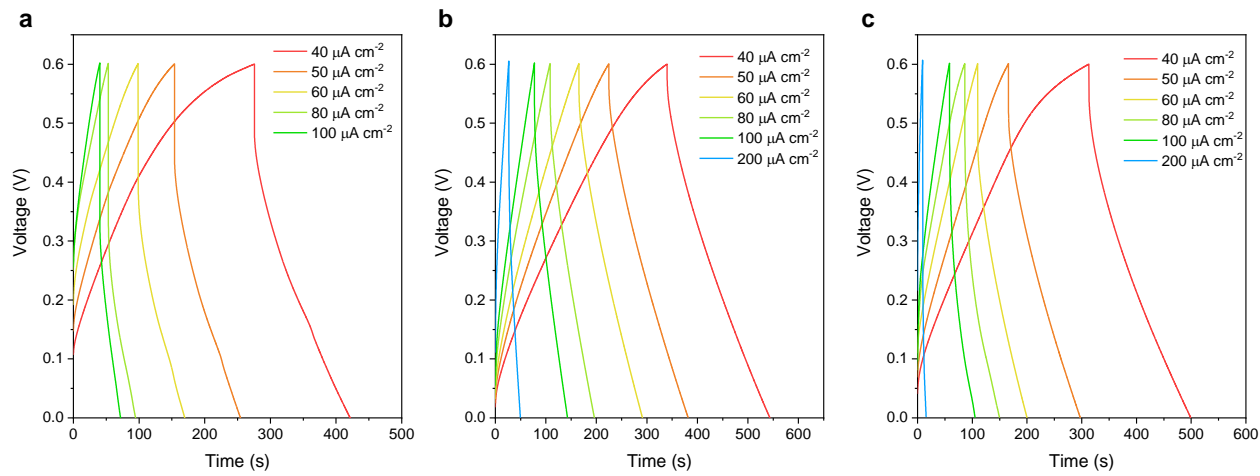

Figure S4.9 GCD curves of 30 SSCs with (a) excess $\mathrm{H}_{2} \mathrm{O}$, (b) external aqueous $5 \mathrm{M} \mathrm{LiCl}$ electrolyte and (c) aqueous $0.5 \mathrm{M} \mathrm{Na}_{2} \mathrm{SO}_{4}$ electrolyte on top at different current densities.

a

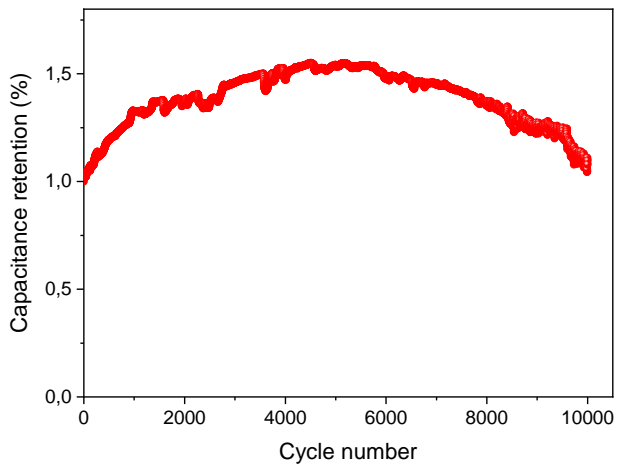

b

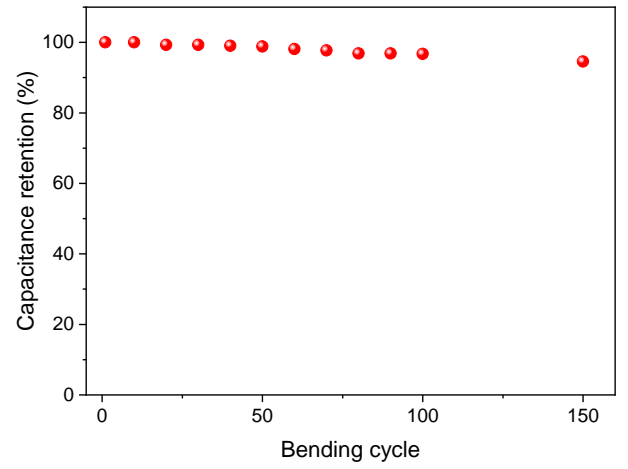

Figure S4.10 (a) Cycling stability of 30L SSC. (b) Cyclic bending test of 30L SSC with a bending radius of about 1 $\mathrm{cm}$ at a current density of $200 \mu \mathrm{A} \mathrm{cm}^{-2}$ and $94.5 \%$ capacitance retention after 150 bending cycles. 
a

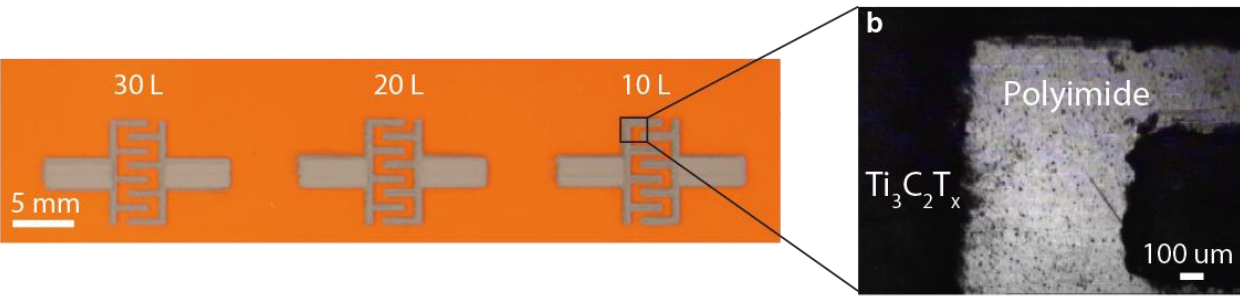

c

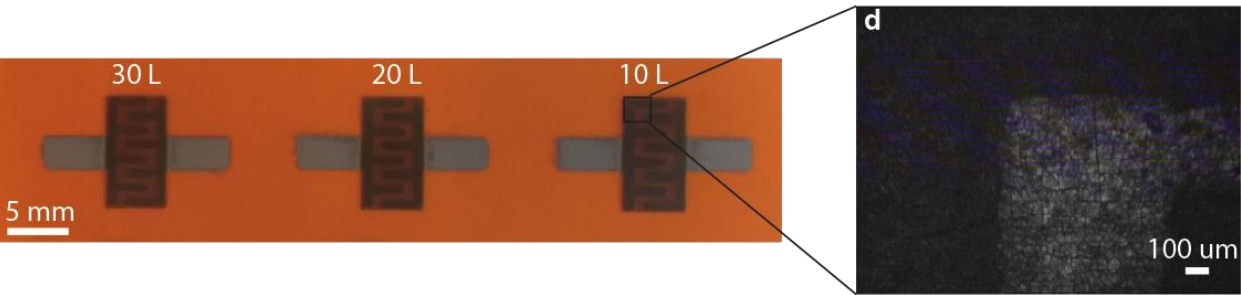

Figure S4.11 Optical image of all-inkjet-printed MSCs. (a) Optical photograph of printed MXene interdigitated electrodes with different layers on polyimide substrate. (b) Zoom-in photograph of printed electrodes from (a) showing the shape edge between phases. (c) Photograph of complete MSCs with GO electrolyte printed on same interdigitated electrode structure as in (a). (d) Zoom-in image of 30 layers MSCs in (c), indicating full coverage of GO electrolyte on top of MXene interdigitated electrodes. The inset codes in (a) and (c) represent the number of printed layers with which the MXene interdigitated electrodes were formed.
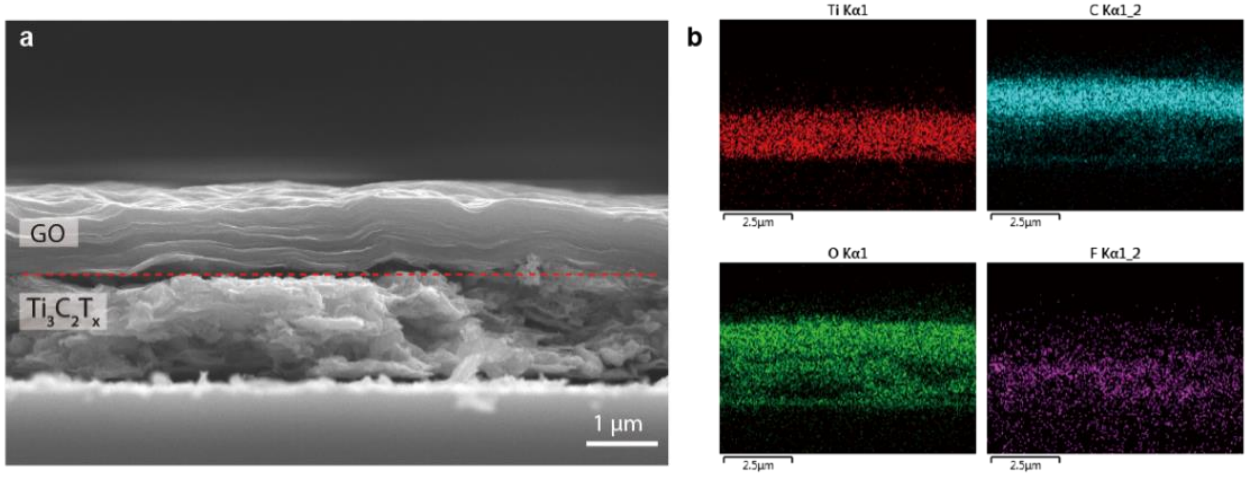

Figure S4.12 (a) Cross-sectional SEM image of all-inkjet-printed MSC showing electrode and electrolyte interface and (b) EDS elemental mappings of the elements Ti, C, O and F. 


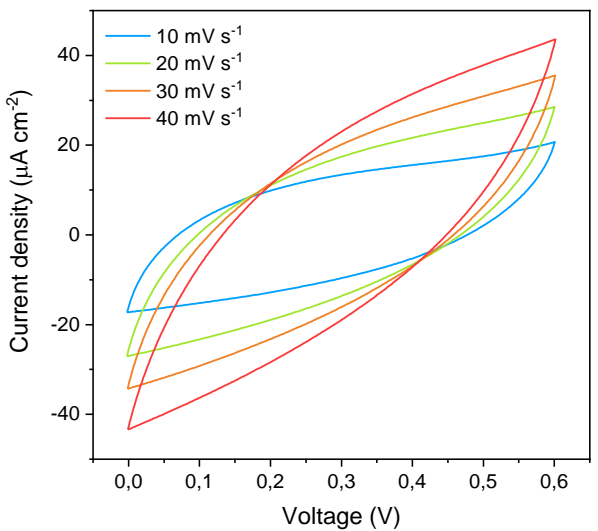

C

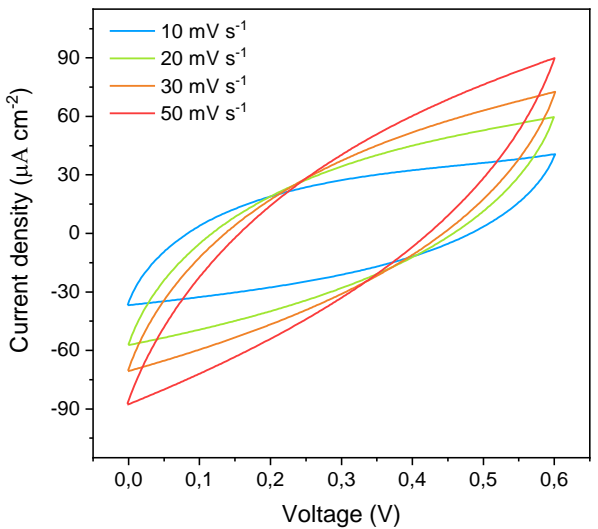

e

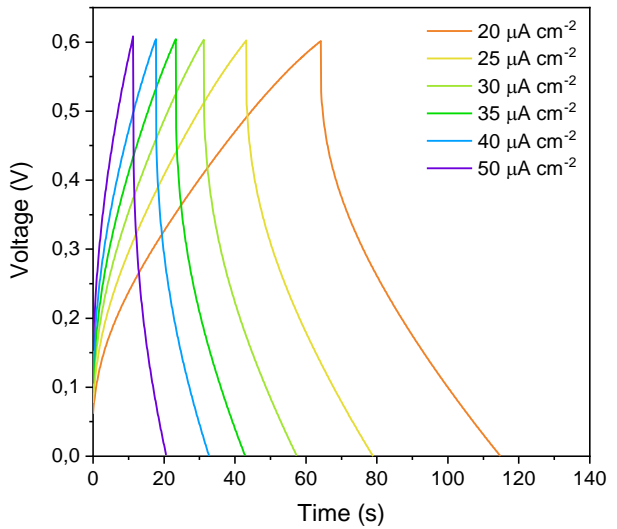

b

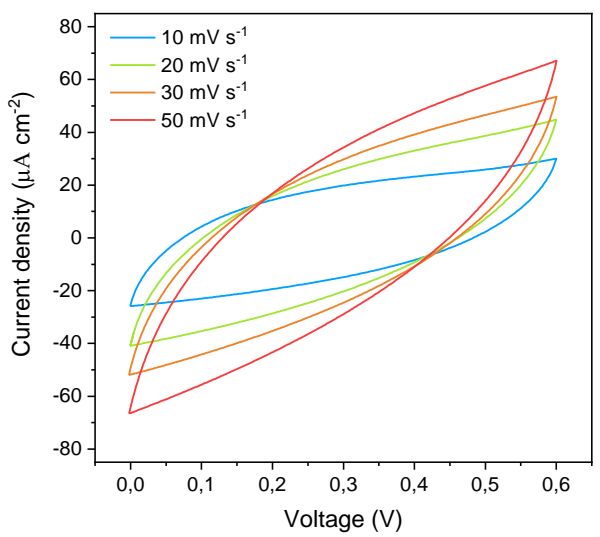

d

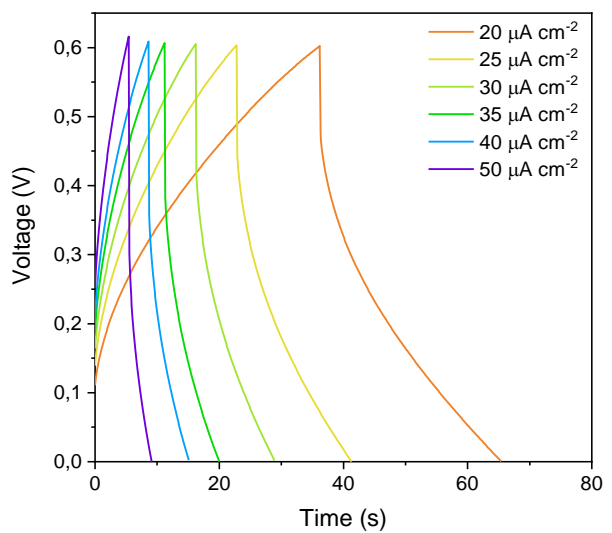

f

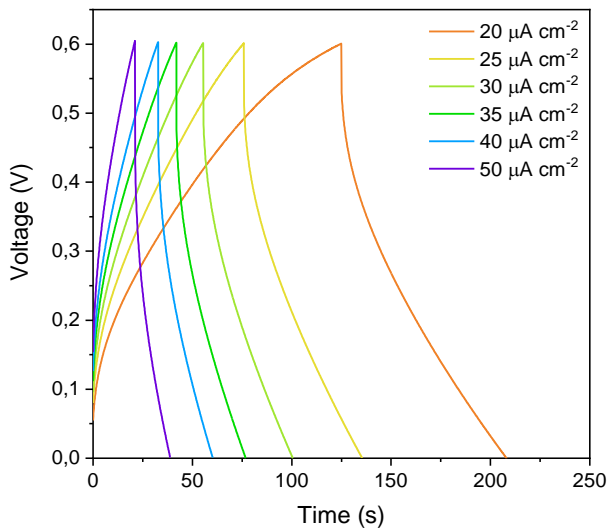

Figure S4.13 CV curves of (a) 10L, (b) 20L and (c) 30L MSCs with excess water at different scan rates. GCD curves of (d) 10L, (e) 20L and (f) 30L MSCs with excess water at different current densities. 

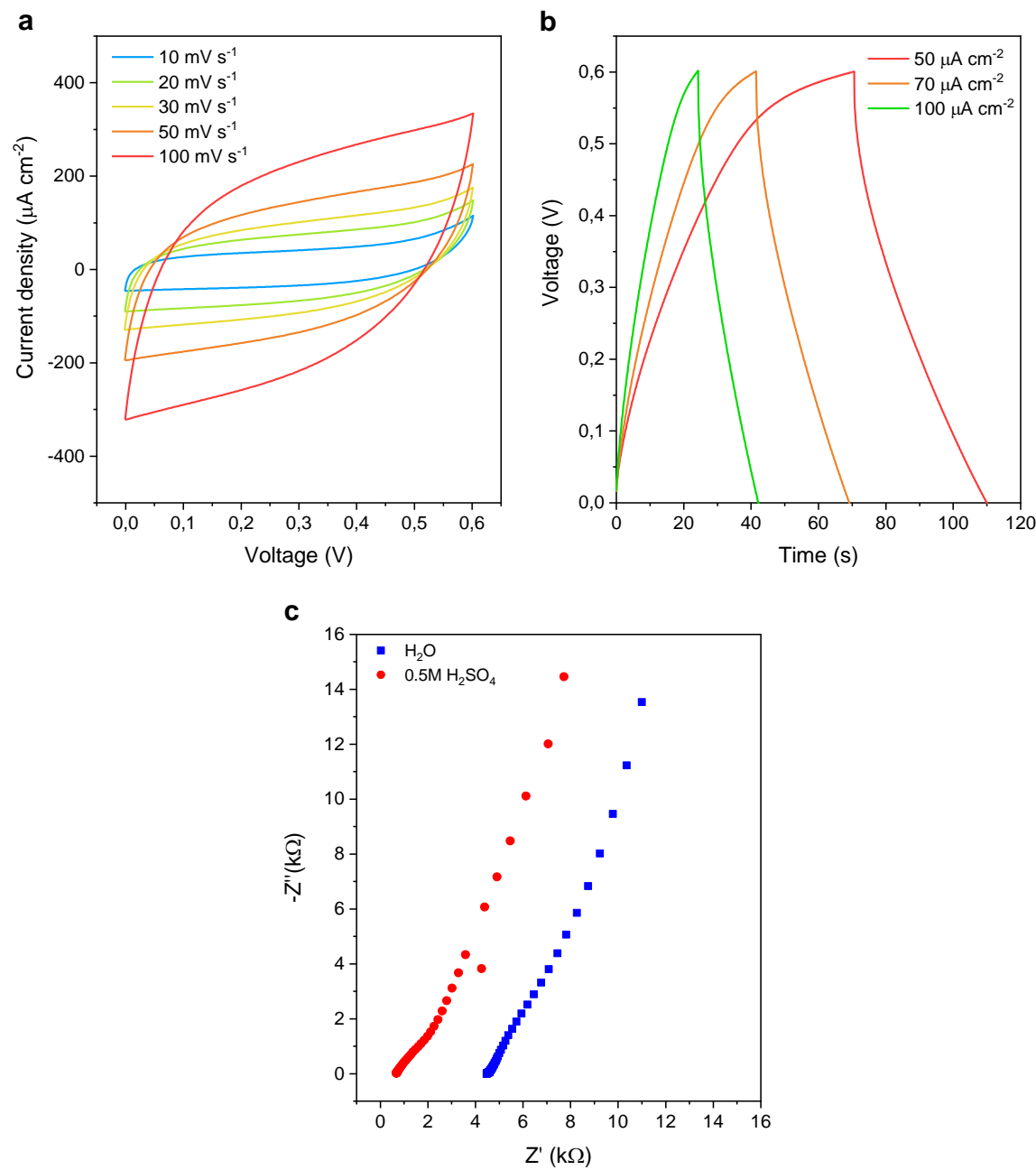

Figure S4.14 (a) CV curves of 30L MSC with $0.5 \mathrm{M} \mathrm{H}_{2} \mathrm{SO}_{4}$ electrolyte at different scan rates. (b) GCD curves of 30L MSC with $0.5 \mathrm{M} \mathrm{H}_{2} \mathrm{SO}_{4}$ electrolyte at different current densities. (c) Nyquist plots of 30L MSC with excess water and with $0.5 \mathrm{M} \mathrm{H}_{2} \mathrm{SO}_{4}$ electrolyte. 
Table S4.1 Components of the equivalent circuits fitted for all-inkjet-printed 10L and 30L SSCs as shown in Figure 4.5c.

\begin{tabular}{llllllllll}
\hline Samples & $\begin{array}{l}\mathrm{R}_{1} \\
(\mathrm{k} \Omega)\end{array}$ & $\begin{array}{l}\mathrm{R}_{2} \\
(\mathrm{k} \Omega)\end{array}$ & $\begin{array}{l}\mathrm{Q}_{1} \\
\left(\mathrm{~S}^{*} \mathrm{~s}^{\mathrm{n}}\right) \\
\left(10^{-4}\right)\end{array}$ & $\mathrm{n}$ & $\begin{array}{l}\mathrm{Q}_{2} \\
\left(\mathrm{~S}^{*} \mathrm{~s}^{\mathrm{n}}\right) \\
\left(10^{-4}\right)\end{array}$ & & $\mathrm{N}$ & $\begin{array}{l}\mathrm{R}_{3} \\
(\mathrm{k} \Omega)\end{array}$ & $\begin{array}{l}\mathrm{Q}_{3} \\
\left(\mathrm{~S}^{*} \mathrm{~s}^{\mathrm{n}}\right) \\
\left(10^{-4}\right)\end{array}$ \\
\hline 10L SSC & 5.9 & 3.9 & 0.8 & 0.57 & 1.6 & 0.89 & 12.9 & 0.9 & 0.82 \\
30L SSC & 2.1 & 3.1 & 1.3 & 0.50 & 2.6 & 1.00 & 1.9 & 2.9 & 0.66 \\
\end{tabular}

Table S4.2 Components of the equivalent circuits fitted for all-inkjet-printed MSCs as shown in Figure 4.9a.

\begin{tabular}{|c|c|c|c|c|c|c|}
\hline Samples & $\begin{array}{l}\text { Chi- } \\
\text { Squared } \\
\left(10^{-6}\right)\end{array}$ & $\begin{array}{l}\mathrm{Q}_{1}\left(\mathrm{~S}^{*} \mathrm{~s}^{\mathrm{n}}\right)\left(10^{-}\right. \\
\left.{ }^{4}\right)\end{array}$ & $\mathrm{n}$ & $\mathrm{R}_{1}(\mathrm{k} \Omega)$ & $\mathrm{Q}_{3}\left(\mathrm{~S}^{*} \mathrm{~s}^{\mathrm{n}}\right)\left(10^{-4}\right)$ & $\mathrm{n}$ \\
\hline 10L MSC & 3.1 & 2.9 & 1.00 & 12.0 & 1.5 & 0.66 \\
\hline 20L MSC & 1.7 & 2.2 & 0.84 & 11.9 & 2.1 & 0.73 \\
\hline 30L MSC & 1.3 & 6.1 & 1.00 & 4.1 & 4.5 & 0.82 \\
\hline
\end{tabular}

Due to the complexity in the high frequency range, we only present the low frequency fitting results as shown in Table S4.2 and Chi-squared which represent the fitting error for high frequency range. 


\section{Chapter 5}

\section{Printed Two-Dimensional $\mathrm{V}_{2} \mathrm{O}_{5} / \mathrm{MXene}$ Heterostructure Cathode for Lithium-Ion Batteries}

Two-dimensional nanosheets show promise as electrode materials for high electrochemical performance lithium-ion batteries owing to their distinguished properties. However, individual nanosheets cannot meet all the required properties in one material for batteries to achieve high performance. Here, we demonstrate two-dimensional heterostructure cathodes for lithium-ion batteries by inkjet printing a high capacity $\mathrm{V}_{2} \mathrm{O}_{5}$ nanosheet and high electronic conductivity $\mathrm{Ti}_{3} \mathrm{C}_{2} \mathrm{~T}_{\mathrm{x}}$ nanosheet-based composite ink. The printed cathodes exhibit a high capacity of $321 \mathrm{mAh} \mathrm{g}^{-1}$ at $1 \mathrm{C}$, high-rate capacities of $112 \mathrm{mAh} \mathrm{g}^{-1}$ at $10.5 \mathrm{C}$ and good cycling stabilities after 680 cycles with $91.8 \%$ capacity retention, indicating high electrochemical performance of the printed heterostructure cathode.

This chapter is in preparation:

Wang, Y.; Lubbers, T.; Xia, R.; Mehrali, M.; Huijben, M.; ten Elshof, J. E., Printed twodimensional $\mathrm{V}_{2} \mathrm{O}_{5} / \mathrm{MXene}$ heterostructure cathode for lithium-ion batteries. (Submitted) 


\subsection{Introduction}

2D materials have recently attracted considerable attention because of their distinct electronic properties, shortened ion diffusion paths and cycling stability for energy storage applications like lithium-ion batteries (LIBs). ${ }^{1}$ In particular, 2D materials exhibit enhanced electrochemical properties because of the increased number of surface active sites and surface area. ${ }^{2} \mathrm{~A}$ wide range of $2 \mathrm{D}$ materials such as graphene, ${ }^{3}$ transition metal dichalcogenides ${ }^{4}$ and transition metal oxides ${ }^{5}$ have been demonstrated as promising electrodes materials for LIBs. Among them, 2D vanadium pentoxide $\left(\mathrm{V}_{2} \mathrm{O}_{5}\right)$ nanosheets show great promise as a cathode material for LIBs because of their high theoretical capacity of $294.8 \mathrm{mAh} \mathrm{g}^{-1}$ with two lithium ion intercalations per unit cell in its structure for a potential window between 2 to $4 \mathrm{~V}{ }^{6}$ However, the intrinsic poor electronic conductivity of $\mathrm{V}_{2} \mathrm{O}_{5}$ nanosheets limits the electrochemical performance. Therefore, individual 2D nanosheets cannot meet all properties to maximize battery electrochemical performance such as energy/power density and cycle life.

2D nanosheets exhibit various interesting electronic properties like metallic conductivity, semiconductivity or insulating behavior. ${ }^{7} \mathrm{We}$ argue that fabricating heterostructure electrodes by stacking different 2D nanosheets will give new opportunities to realize high electrochemical performance electrodes by combing the advantages of different 2D nanosheet building blocks while eliminating their limitations. ${ }^{8}$

MXene is a new class of $2 D$ materials with general formula $M_{n+1} X_{n} T_{x}$, where $M$ is an early transition metal, $\mathrm{X}$ is $\mathrm{C}$ or $\mathrm{N}, \mathrm{T}_{x}$ is a surface termination functional group such as $-\mathrm{OH},-\mathrm{O}$ or $-\mathrm{F}$, and $\mathrm{n}=1,2,3,4 .{ }^{9}$ MXene nanosheets that exhibit excellent electronic conductivity, high specific surface area and hydrophilicity shows promise as charge transport materials. ${ }^{10}$ Moreover, water-based MXene nanosheet suspensions can be used as inks for versatile, digital and low-cost inkjet printing. $\mathrm{Ti}_{3} \mathrm{C}_{2} \mathrm{~T}_{\mathrm{x}}$ nanosheets, which have been widely studied, are reported in this work.

Here we show the fabrication of a thin-film heterostructure cathode by inkjet printing a waterbased $\mathrm{V}_{2} \mathrm{O}_{5} / \mathrm{Ti}_{3} \mathrm{C}_{2} \mathrm{~T}_{\mathrm{x}}$ nanosheet composite ink. The novel printed heterostructure cathode exhibited high capability to accommodate large volume changes during electrochemical measurements, resulting in a high capacity and long cycling life.

\subsection{Experimental Section}

\subsubsection{Preparation of $\mathrm{V}_{2} \mathrm{O}_{5}$ nanosheets}

In order to prepare $\mathrm{V}_{2} \mathrm{O}_{5}$ nanosheets, $\mathrm{V}_{2} \mathrm{O}_{5}$ powder was firstly reduced to $\mathrm{VO}_{2}(\mathrm{~B})$ nanosheets followed by oxidation into $\mathrm{V}_{2} \mathrm{O}_{5}$ nanosheets by thermal annealing in air. $\mathrm{VO}_{2}$ (B) was synthesized by hydrothermal reaction as described elsewhere. ${ }^{11}$ The reaction mixture $(10 \mathrm{~mL})$ was prepared by dispersing $20 \mathrm{mg} \mathrm{V} \mathrm{O}_{5}$ powder (Alfa Aesar >99.6\%) in $2 \mathrm{~mL}$ deionized (DI) 
water by ultrasonication treatment for 5 minutes. Then, $4 \mathrm{~mL} \mathrm{H}_{2} \mathrm{O}_{2}\left(\mathrm{Sigma} 30 \%\right.$ in $\left.\mathrm{H}_{2} \mathrm{O}\right)$ was added whilst stirring vigorously. The dispersion was stirred for another 5 minutes resulting in a clear yellow solution. $4 \mathrm{~mL}$ isopropyl alcohol (IPA, Boom, technical grade) was added and stirred for another 5 minutes. The addition of IPA was accompanied by mild oxygen bubbling, which was generated from the solution. Then, a certain amount of reaction mixture was placed in an autoclave reactor vessel which was then heated to $180{ }^{\circ} \mathrm{C}$ for $6 \mathrm{~h}$. The reacted suspension was washed with ethanol three times by centrifuging for 10 minutes at $8000 \mathrm{rpm}$. The collected dark blue precipitate was then re-dispersed in DI water by ultrasonication for one hour to make sure that all individual nanostructures were separated as good as possible. The suspension was then filtered on a flat filter paper using vacuum filtration. The filter paper with materials was dried in an oven at $80{ }^{\circ} \mathrm{C}$ for at least 2 hours to obtain dried $\mathrm{VO}_{2}$ (B). The dried $\mathrm{VO}_{2}$ (B) thin film was peeled off and then calcined in a tube oven for 2 hours at $350{ }^{\circ} \mathrm{C}$ with a heating ramp rate of $2{ }^{\circ} \mathrm{C} \mathrm{min}{ }^{-1}$ to get $\mathrm{V}_{2} \mathrm{O}_{5}$ sample.

\subsubsection{Preparation of $\mathrm{Ti}_{3} C_{2} T_{x}$ nanosheets suspension}

The detailed preparation of $\mathrm{Ti}_{3} \mathrm{C}_{2} \mathrm{~T}_{\mathrm{x}}$ nanosheet suspensions was described in Chapter 4.2.1.

\subsubsection{Inkjet printing}

The traditional method to fabricate $\mathrm{V}_{2} \mathrm{O}_{5}$ cathodes is by mixing $\mathrm{V}_{2} \mathrm{O}_{5}$ : conductive agent : binder in a mass ratio of $7: 2: 1$, so the mass ratio of $8: 2$ for $\mathrm{V}_{2} \mathrm{O}_{5}: \mathrm{Ti}_{3} \mathrm{C}_{2} \mathrm{~T}_{\mathrm{x}}$ was used in this study. A printable ink was prepared by dispersing $\mathrm{V}_{2} \mathrm{O}_{5}$ nanosheets into a $\mathrm{Ti}_{3} \mathrm{C}_{2} \mathrm{~T}_{\mathrm{x}}$ nanosheet suspension in a mass ratio of 8:2 followed by ultrasonication to get a homogeneous suspension. All electrodes were inkjet printed onto oxygen plasma treated stainless steel foil substrates with a drop spacing of $20 \mu \mathrm{m}$ at $30{ }^{\circ} \mathrm{C}$ by a Dimatix DMP-2800 inkjet printer (Fujifilm Dimatix), which was equipped with a $10 \mathrm{pL}$ cartridge (DMC-11610). The printed electrodes were dried in a vacuum oven at $60^{\circ} \mathrm{C}$ overnight.

\subsubsection{Electrochemical characterization}

For electrochemical characterization the printed $\mathrm{V}_{2} \mathrm{O}_{5} / \mathrm{Ti}_{3} \mathrm{C}_{2} \mathrm{~T}_{\mathrm{x}}$ cathode was assembled with a lithium metal anode and a glass fiber separator in an electrochemical EL-CELL in an argon atmosphere glovebox $\left(<0.1 \mathrm{ppm}\right.$ of $\mathrm{H}_{2} \mathrm{O}$ and $\left.\mathrm{O}_{2}\right) \cdot 1 \mathrm{M} \mathrm{LiPF}_{6}$ in $1: 1$ ethylene carbonate dimethyl carbonate (EC: DMC) was used as electrolyte. All electrochemical measurements were conducted at room temperature using a BioLogic VMP-300 system in a two-electrode setup. The cyclic voltammetry (CV) and galvanostatic charge/discharge experiments were performed between 2 and $4 \mathrm{~V}$. Only the mass of active material was considered in the capacity calculation.

For ex-situ X-ray photoelectron spectroscopy (XPS) spectra, the LIBs were discharged and stopped at specific potentials related to the electrochemical reactions. The LIBs were then transferred into a glovebox for disassembly. The electrodes were rinsed with dimethyl 
carbonate (Aldrich, 99.9\%) to remove excel electrolyte. Finally, the electrodes were transferred to an XPS chamber within a short time.

\subsubsection{Materials characterization}

X-ray diffraction (XRD) analysis was done with a PANalytical X'Pert Pro with filtered $\mathrm{Cu}$ $\mathrm{K} \alpha$ radiation $(\lambda=0.15405 \mathrm{~nm})$. XPS spectra wre recorded using an Omicron Nanotechnology $\mathrm{GmbH}$ (Oxford Instruments) surface analysis system with a photon energy of $1486.7 \mathrm{eV}$ (Al $\mathrm{K} \alpha \mathrm{X}$-ray source) with a scanning step size of $0.1 \mathrm{eV}$. The pass energy was set to $20 \mathrm{eV}$. The spectra were corrected using the binding energy of $\mathrm{C} 1 s$ of adventitious carbon as a reference. Atomic Force Microscopy (AFM) (Veeco Dimension Icon) was conducted in standard tapping mode. The AFM data were analyzed by Gwyddion (version 2.47) software. High resolution scanning electron microscopy (HRSEM; Zeiss MERLIN) was performed to acquire information of $\mathrm{V}_{2} \mathrm{O}_{5}$ nanosheets and printed $\mathrm{V}_{2} \mathrm{O}_{5} / \mathrm{Ti}_{3} \mathrm{C}_{2} \mathrm{~T}_{\mathrm{x}}$ electrode.

\subsection{Results and discussion}

\subsubsection{Structure characterization}

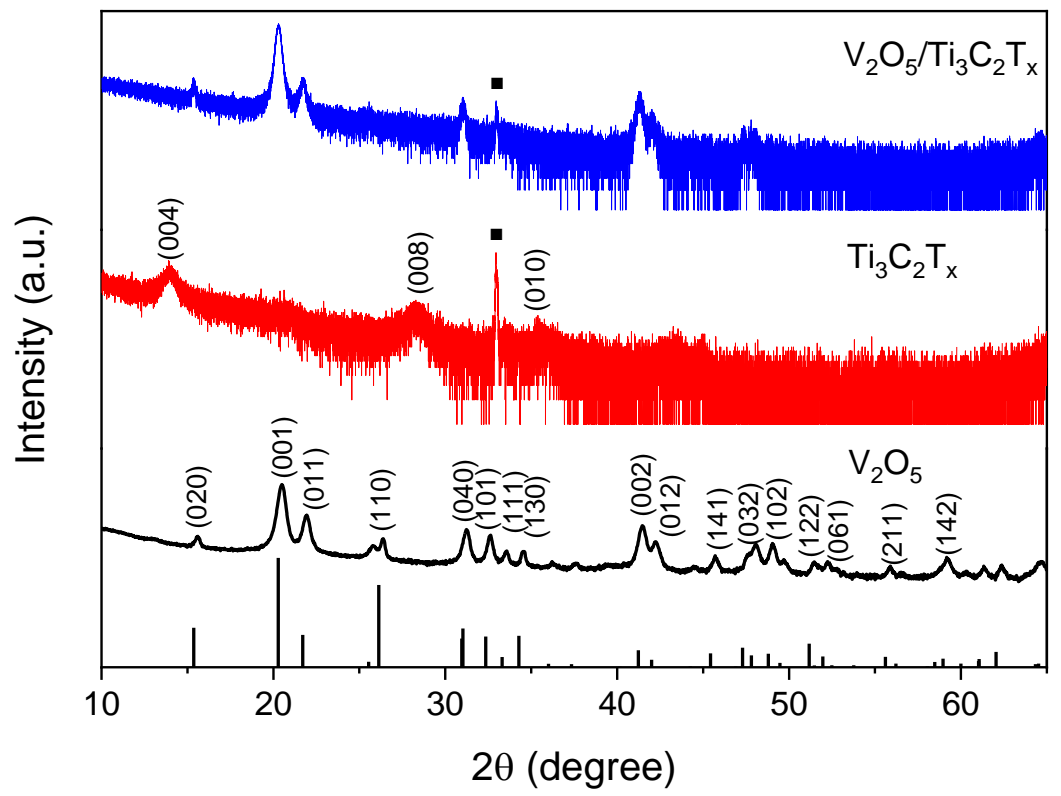

Figure 5.1 XRD patterns of $2 \mathrm{D} \mathrm{V}_{2} \mathrm{O}_{5}, \mathrm{Ti}_{3} \mathrm{C}_{2} \mathrm{~T}_{\mathrm{x}}$ and printed $\mathrm{V}_{2} \mathrm{O}_{5} / \mathrm{Ti}_{3} \mathrm{C}_{2} \mathrm{~T}_{\mathrm{x}}$ film. The black symbols represent peaks from silicon substrates.

The color of the $\mathrm{V}_{2} \mathrm{O}_{5}$ solution changed from orange to bright yellow after adding $\mathrm{H}_{2} \mathrm{O}_{2}$, indicating the formation of a $\mathrm{V}_{2} \mathrm{O}_{5}$ sol. ${ }^{11,12}$ The addition of isopropanol into the solution was 
done to reduce $\mathrm{V}_{2} \mathrm{O}_{5}$ to $\mathrm{VO}_{2}$ (B) species with a layered structure. ${ }^{11}$ The $\mathrm{VO}_{2}$ (B) nanosheets were thermally annealed at $350{ }^{\circ} \mathrm{C}$ in the air for $2 \mathrm{~h}$ to obtain $\mathrm{V}_{2} \mathrm{O}_{5}$ nanosheets. XRD analysis was performed to determine the crystal structure and phase information of the as-prepared $\mathrm{VO}_{2}$ and $\mathrm{V}_{2} \mathrm{O}_{5}$ nanosheets. As shown in Figure S5.1 of the Appendix, the obtained $\mathrm{VO}_{2}(\mathrm{~B})$ nanosheets were in good accordance with monoclinic $\mathrm{VO}_{2}$ (B) (JCPDS card no. 81-2392, space group $C 2 / m$, No. $\left.12, a=12.05 \AA, b=3.70 \AA, c=6.41 \AA, \beta=106.86^{\circ}\right) .{ }^{13}$ The reflection of the $(00 l)$ series peaks indicate the lamellar and ultrathin features of the prepared sample. In particular, the $(00 l)$ series peaks have a high intensity compared to the other peaks, and the (010) peak is almost completely absent. The relative intensity change compared to the JCPDS card reference is probably due to alignment of the nanostructures. ${ }^{11}$ The XRD pattern of the $\mathrm{V}_{2} \mathrm{O}_{5}$ nanosheets is shown in Figure 5.1a. The spectrum is in good accordance with orthorhombic $\mathrm{V}_{2} \mathrm{O}_{5}$ (JCPDS card no. 74-4605, space group Pmmn, No. 59, $a=11.46 \AA$, $b=4.36 \AA, c=3.57 \AA) .{ }^{14}$ The $(00 l)$ series reflections are relatively intense compared to the expected intensity described in the card. Examples of peaks with much lower intensities compared to the JCPDS card values are the (020) reflection at $2 \theta=15.5^{\circ}$ and the (110) reflection at $2 \theta=26.2^{\circ}$. The XRD diffractogram of printed $\mathrm{Ti}_{3} \mathrm{C}_{2} \mathrm{~T}_{\mathrm{x}}$ film shows the $(004)$ peak at $2 \theta=14.1^{\circ}$ indicating a high degree of ordering in the $c$ direction. For printed $\mathrm{V}_{2} \mathrm{O}_{5} /$ $\mathrm{Ti}_{3} \mathrm{C}_{2} \mathrm{~T}_{\mathrm{x}}$ heterostructure films, the $(00 l)$ peaks are pronounced suggesting a well-defined $c$ orientation of the heterostructure electrode with stacked nanosheets. However, the presence of small (011), (040) and (012) peaks indicate that exfoliation of $\mathrm{V}_{2} \mathrm{O}_{5}$ nanosheets was not entirely completed. Moreover, $\mathrm{Ti}_{3} \mathrm{C}_{2} \mathrm{~T}_{\mathrm{x}}$ nanosheets peaks are absent indicating that they are completely dispersed without aggregation.

a

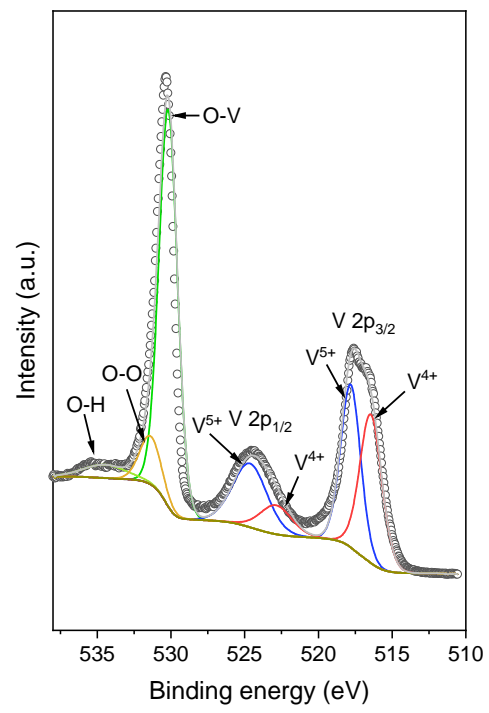

b

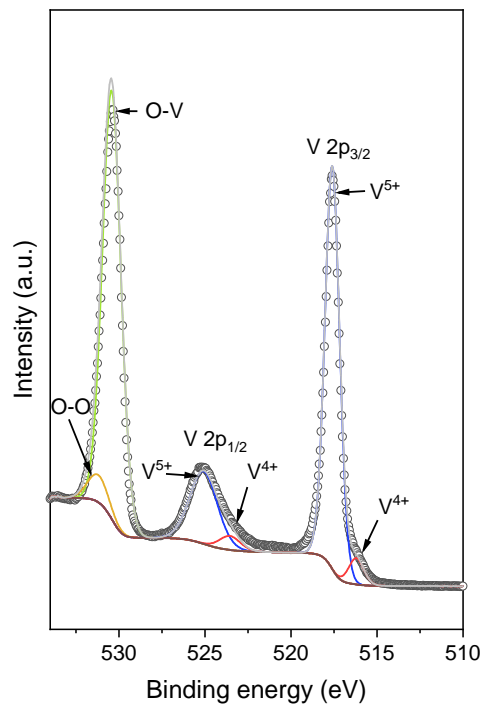

Figure 5.2 High-resolution XPS spectra of (a) $\mathrm{VO}_{2}$ (B) and (b) $\mathrm{V}_{2} \mathrm{O}_{5}$ nanosheets. 
The high-resolution XPS spectra were recorded to obtain oxidation state information on the prepared $\mathrm{VO}_{2}(\mathrm{~B})$ and $\mathrm{V}_{2} \mathrm{O}_{5}$ nanosheets. Figure 5.2a shows two binding energy (BE) peaks of vanadium in $\mathrm{VO}_{2}(\mathrm{~B})$, which can be assigned to $\mathrm{V} 2 p_{3 / 2}$ and $\mathrm{V} 2 p_{1 / 2}$, respectively. The $\mathrm{BE}$ of $\mathrm{V} 2 p_{3 / 2}$ at 517.8 and $516.4 \mathrm{eV}$ can be attributed to the $\mathrm{V}^{5+}$ and $\mathrm{V}^{4+}$ oxidation states, respectively. Note that $\mathrm{VO}_{2}(\mathrm{~B})$ is expected to have only $\mathrm{V}^{4+}$ while the XPS shows that the surface also contains $\mathrm{V}^{5+}$ in approximately a 50:50\% ratio. The presence of the $\mathrm{V}^{5+}$ component is probably due to the existence of $\mathrm{V}_{2} \mathrm{O}_{5}$ at the surface of $\mathrm{VO}_{2}(\mathrm{~B})$ resulting from exposure to air. ${ }^{15}$ The high resolution spectrum of $\mathrm{O} 1 s$ at $530.2,531.4$ and $534.5 \mathrm{eV}$ corresponds to the presence of $\mathrm{O}-\mathrm{V}, \mathrm{O}-\mathrm{O}$ and $\mathrm{O}-\mathrm{H}$ groups, respectively. As shown in Figure $5.2 \mathrm{~b}$, the main peak $\mathrm{BE}$ of $\mathrm{V} 2 p_{3 / 2}$ at $517.6 \mathrm{eV}$ and the small peak at $516.2 \mathrm{eV}$ in $\mathrm{V}_{2} \mathrm{O}_{5}$ correspond to $\mathrm{V}^{5+}$ and $\mathrm{V}^{4+}$, respectively. ${ }^{16}$ The $\mathrm{BE}$ difference between the $\mathrm{O} 1 s$ and $\mathrm{V} 2 p_{3 / 2}$ levels for the $\mathrm{V}^{5+}$ is $12.8 \mathrm{eV}$ which identical to the reported value. ${ }^{17}$ Similar to $\mathrm{VO}_{2}(\mathrm{~B})$, peaks in the high resolution spectrum of $\mathrm{O} 1 s$ at 530.4 and $531.2 \mathrm{eV}$ correspond to the $\mathrm{O}-\mathrm{V}$ and $\mathrm{O}-$ O groups, respectively.
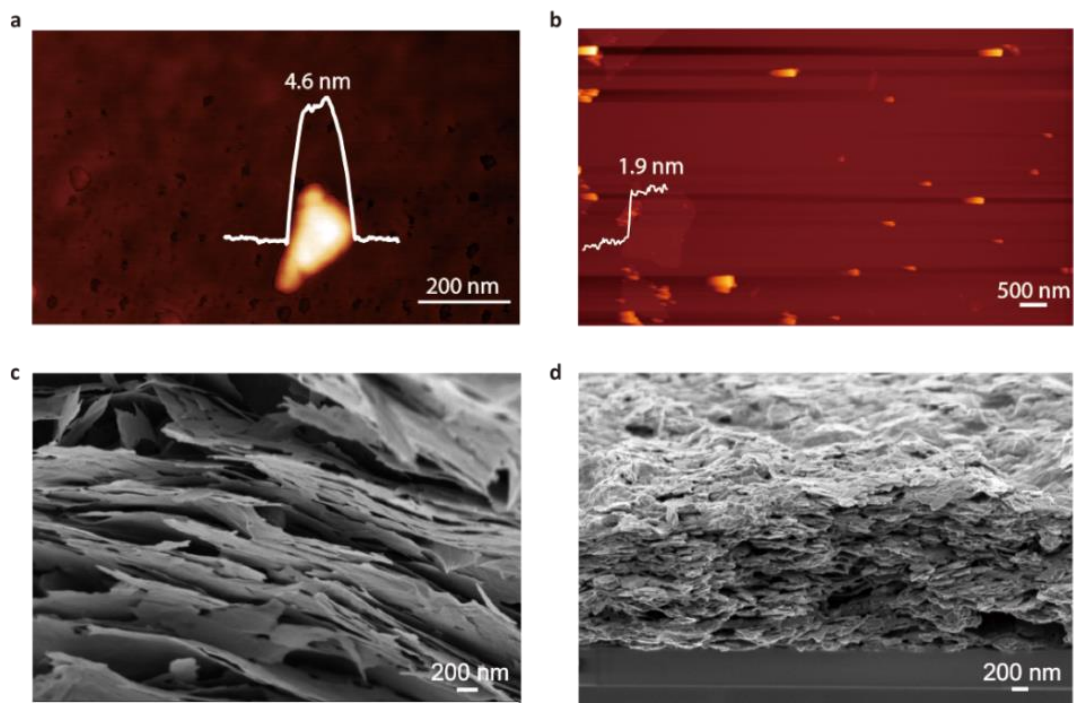

Figure 5.3 AFM images of (a) $\mathrm{V}_{2} \mathrm{O}_{5}$ nanosheet and (b) $\mathrm{Ti}_{3} \mathrm{C}_{2} \mathrm{~T}_{\mathrm{x}}$ nanosheet. Cross-sectional SEM images of (c) vacuum filtrated $\mathrm{V}_{2} \mathrm{O}_{5}$ film and (d) inkjet printed $\mathrm{V}_{2} \mathrm{O}_{5} / \mathrm{Ti}_{3} \mathrm{C}_{2} \mathrm{~T}_{\mathrm{x}}$ film.

The thickness of $\mathrm{V}_{2} \mathrm{O}_{5}$ nanosheets was determined by AFM to be around $4.6 \mathrm{~nm}$, which is in agreement with reported data, indicating an ultra-thin structure with several layers (Figure 5.3a). ${ }^{11}$ The thickness of the $\mathrm{Ti}_{3} \mathrm{C}_{2} \mathrm{~T}_{\mathrm{x}}$ nanosheet is about $1.9 \mathrm{~nm}$, suggesting a unilamellar structure (Figure 5.3b). The thickness difference between the measured unilamellar $\mathrm{Ti}_{3} \mathrm{C}_{2} \mathrm{~T}_{\mathrm{x}}$ nanosheet and the theoretical thickness, which is $0.98 \mathrm{~nm}$, can be explained by the presence of molecular surface water. ${ }^{18}$ The cross-sectional SEM image of a $\mathrm{V}_{2} \mathrm{O}_{5}$ nanosheet film 
(Figure 5.3c) shows a high concentration of nanosheets present in lamellar structure, and even single sheets and agglomerated sheets can be distinguished. The prepared water-based $\mathrm{V}_{2} \mathrm{O}_{5} / \mathrm{Ti}_{3} \mathrm{C}_{2} \mathrm{~T}_{\mathrm{x}}$ composite ink shows a high quality viscoelastic behavior which was confirmed by the optical images of droplet formation versus time as shown in Figure S5.2 (Appendix). The cross-sectional SEM image of a printed $\mathrm{V}_{2} \mathrm{O}_{5} / \mathrm{Ti}_{3} \mathrm{C}_{2} \mathrm{~T}_{\mathrm{x}}$ electrode on silicon substrate shows a high degree of orientation with a layer-by-layer structure (Figure 5.3d). Moreover, the printed electrode shows continuity coverage over a large area (Figure S5.3, Appendices), indicating the reliability of inkjet printing to fabricate thin film electrodes.

\subsubsection{Electrochemical measurements}

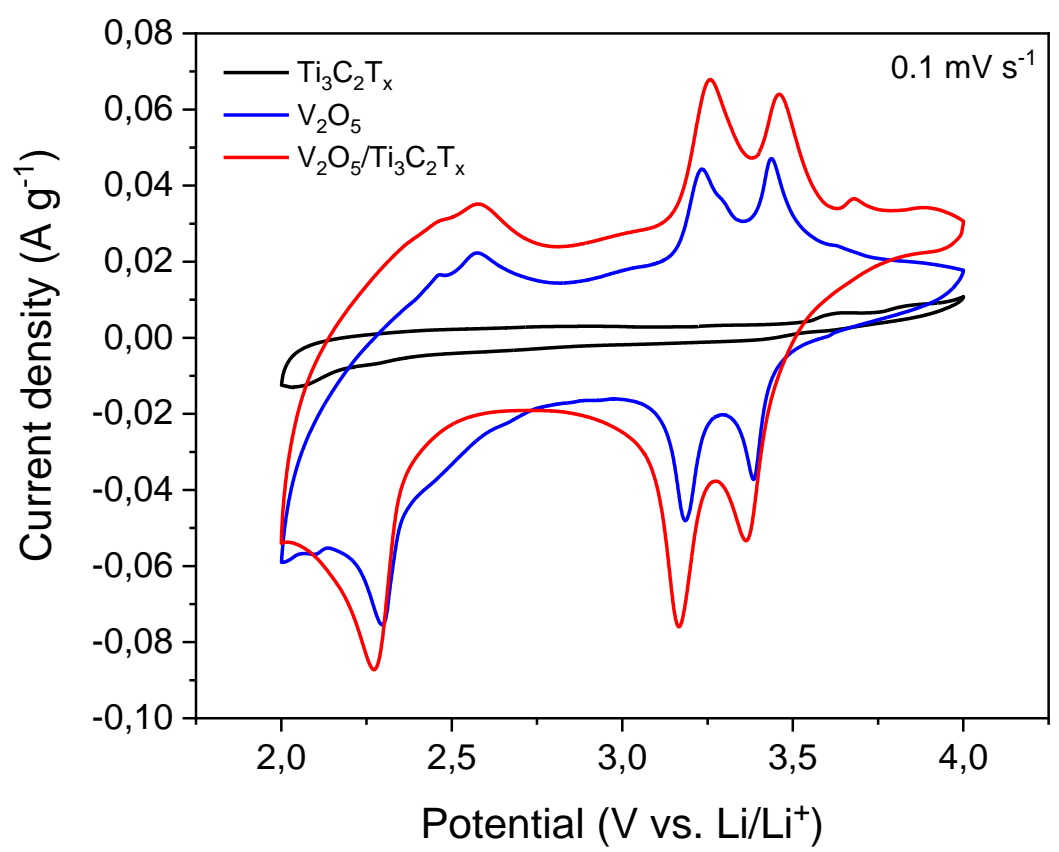

Figure 5.4 CV profiles of printed $\mathrm{V}_{2} \mathrm{O}_{5} / \mathrm{Ti}_{3} \mathrm{C}_{2} \mathrm{~T}_{\mathrm{x}}$ cathode, printed $\mathrm{V}_{2} \mathrm{O}_{5}$ nanosheet cathode and printed $\mathrm{Ti}_{3} \mathrm{C}_{2} \mathrm{~T}_{\mathrm{x}}$ cathode at a scan rate of $0.1 \mathrm{mV} \mathrm{s}^{-1}$ between 2 and $4 \mathrm{~V}$ in half-cells.

The electrochemical performances of a printed $\mathrm{V}_{2} \mathrm{O}_{5} / \mathrm{Ti}_{3} \mathrm{C}_{2} \mathrm{~T}_{\mathrm{x}}$ cathode, a printed $\mathrm{V}_{2} \mathrm{O}_{5}$ nanosheet cathode and a printed $\mathrm{Ti}_{3} \mathrm{C}_{2} \mathrm{~T}_{\mathrm{x}}$ electrode were studied in half-cell configuration with lithium metal as anode. As shown in Figure 5.4, the printed $\mathrm{Ti}_{3} \mathrm{C}_{2} \mathrm{~T}_{\mathrm{x}}$ electrode shows a small current response in a $\mathrm{CV}$ profile compared with a $\mathrm{V}_{2} \mathrm{O}_{5} / \mathrm{Ti}_{3} \mathrm{C}_{2} \mathrm{~T}_{\mathrm{x}}$ cathode at a scan rate of $0.1 \mathrm{mV} \mathrm{s}^{-1}$, indicating that $\mathrm{Ti}_{3} \mathrm{C}_{2} \mathrm{~T}_{\mathrm{x}}$ make a small contribution to the total current response. The $\mathrm{V}_{2} \mathrm{O}_{5} / \mathrm{Ti}_{3} \mathrm{C}_{2} \mathrm{~T}_{\mathrm{x}}$ cathode exhibited a higher current density than the printed $\mathrm{V}_{2} \mathrm{O}_{5}$ nanosheets cathode, suggesting that the addition of $\mathrm{Ti}_{3} \mathrm{C}_{2} \mathrm{~T}_{\mathrm{x}}$ nanosheets enhanced the charge transport. Moreover, the $1^{\text {st }}$ to $4^{\text {th }} \mathrm{CV}$ curves of a printed $\mathrm{V}_{2} \mathrm{O}_{5}$ nanosheet cathode at a scan 

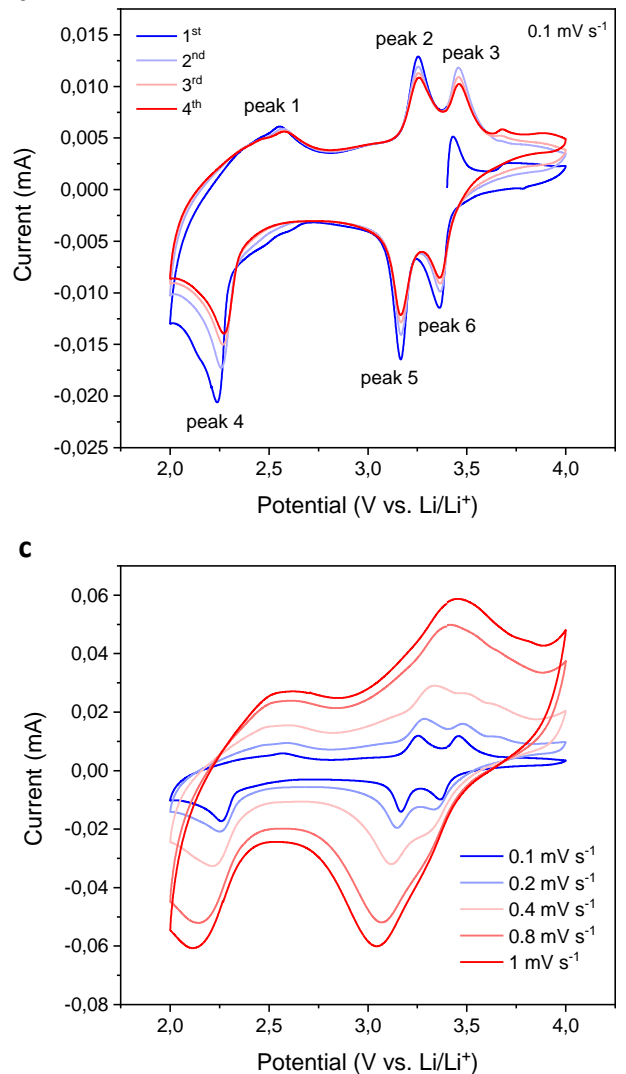

e

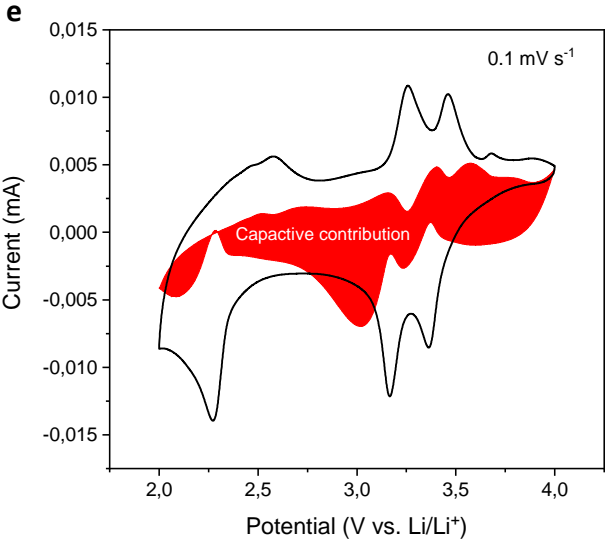

b

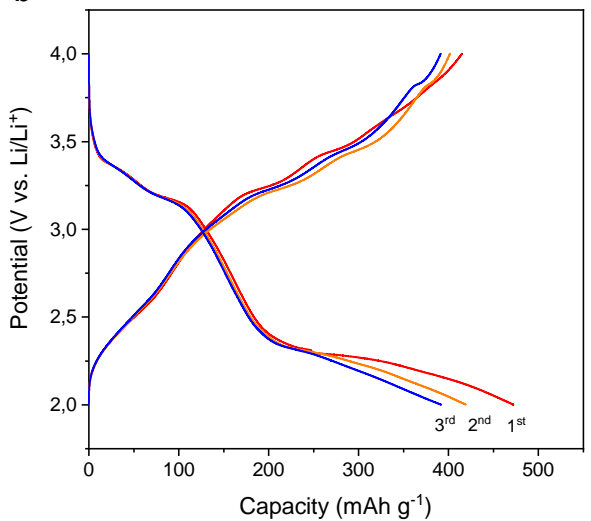

d

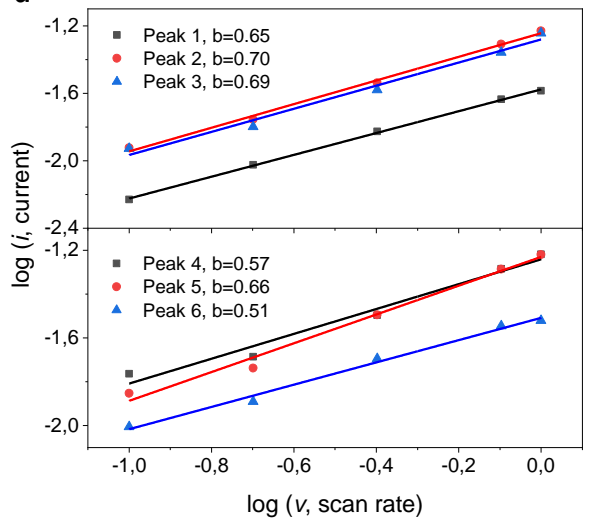

f

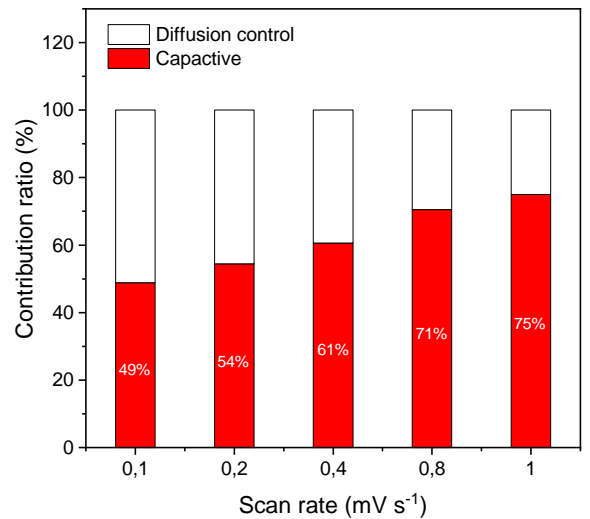

Figure 5.5 Electrochemical analysis of a printed $\mathrm{V}_{2} \mathrm{O}_{5} / \mathrm{Ti}_{3} \mathrm{C}_{2} \mathrm{~T}_{\mathrm{x}}$ cathode in a half-cell. (a) First to fourth cycle of voltammetric sweep at a scan rate of $0.1 \mathrm{mV} \mathrm{s}^{-1}$ between 2 and $4 \mathrm{~V}$. (b) First to third cycle galvanostatic charge/discharge curves at $0.5 \mathrm{C}$. (c) $\mathrm{CV}$ sweep of printed $\mathrm{V}_{2} \mathrm{O}_{5} / \mathrm{Ti}_{3} \mathrm{C}_{2} \mathrm{~T}_{\mathrm{x}}$ cathodes from 0.1 to $1 \mathrm{mV} \mathrm{s}{ }^{-1}$. (d) $\mathrm{The} \log (i)$ versus $\log (v)$ plot of the charge peaks (above) and discharge peaks (bottom). (e) $k_{1}, k_{2}$ analysis of printed $\mathrm{V}_{2} \mathrm{O}_{5} / \mathrm{Ti}_{3} \mathrm{C}_{2} \mathrm{~T}_{\mathrm{x}}$ cathodes at $0.1 \mathrm{mV} \mathrm{s}^{-1}$. The red area shows the contribution of surface capacitance as a function of 
potential. (f) The surface capacitive contribution and diffusion control ratio at different scan rates.

rate of $0.2 \mathrm{mV} \mathrm{s}^{-1}$ showed current decrease for redox peak at 2.3 V (Figure S5.4, Appendix), while the $\mathrm{CV}$ curves of the printed $\mathrm{V}_{2} \mathrm{O}_{5} / \mathrm{Ti}_{3} \mathrm{C}_{2} \mathrm{~T}_{\mathrm{x}}$ cathode almost overlapped at a scan rate of $0.2 \mathrm{mV} \mathrm{s}^{-1}$ (Figure S5.5, Appendix), further indicating that the addition of $\mathrm{Ti}_{3} \mathrm{C}_{2} \mathrm{~T}_{\mathrm{x}}$ nanosheets into $\mathrm{V}_{2} \mathrm{O}_{5}$ materials improved the cathode's electrochemical performance. The large current response of the printed $\mathrm{V}_{2} \mathrm{O}_{5} / \mathrm{Ti}_{3} \mathrm{C}_{2} \mathrm{~T}_{\mathrm{x}}$ cathode was mainly due to $\mathrm{V}_{2} \mathrm{O}_{5}$ nanosheets which show multiple redox peaks at around 3.4, 3.2, $2.3 \mathrm{~V}$ and 2.6, 3.3 and $3.5 \mathrm{~V}$ from lithium intercalation and deintercalation into $\mathrm{V}_{2} \mathrm{O}_{5}$ nanosheets, respectively.

Figure 5.5a shows the $1^{\text {st }}$ to $4^{\text {th }}$ cycle of the CV curves of printed cathode at a scan rate of 0.1 $\mathrm{mV} \mathrm{s}{ }^{-1}$ within the potential range $2-4 \mathrm{~V}\left(\mathrm{vs} . \mathrm{Li} / \mathrm{Li}^{+}\right)$. Three anodic peaks corresponding to the first lithium ion deintercalation of $\gamma / \delta$ (peak 1), the second lithium ion deintercalation of $\delta / \varepsilon$ (peak 2) and $\varepsilon / \alpha$ (peak 3 ) phase transitions at equilibrium potential at around $2.6 \mathrm{~V}, 3.3 \mathrm{~V}$ and $3.5 \mathrm{~V}$, respectively. The three cathodic peaks corresponding to the first lithium ion intercalation of $\alpha / \varepsilon$ (peak 6) and $\varepsilon / \delta$ (peak 5), the second lithium ion intercalation of $\delta / \gamma$ (peak 4) phase transitions at equilibrium potentials at around 3.4, 3.2 and $2.3 \mathrm{~V}$, respectively. ${ }^{6}$ The below reactions show the lithium ion intercalation steps:

$\alpha-\mathrm{V}_{2} \mathrm{O}_{5}+0.5 \mathrm{Li}^{+}+0.5 \mathrm{e}^{-} \leftrightarrow \varepsilon-\mathrm{Li}_{0.5} \mathrm{~V}_{2} \mathrm{O}_{5}$

$\varepsilon-\mathrm{Li}_{0.5} \mathrm{~V}_{2} \mathrm{O}_{5}+0.5 \mathrm{Li}^{+}+0.5 \mathrm{e}^{-} \leftrightarrow \delta-\mathrm{LiV}_{2} \mathrm{O}_{5}$

$\delta-\mathrm{LiV}_{2} \mathrm{O}_{5}+\mathrm{Li}^{+}+\mathrm{e}^{-} \leftrightarrow \gamma-\mathrm{Li}_{2} \mathrm{~V}_{2} \mathrm{O}_{5}$

It is noticeable that the phase transitions of $\mathrm{V}_{2} \mathrm{O}_{5}$ nanosheets during lithium ion intercalation/deintercalation coincide completely with the bulk materials. The peak current values decrease from the $1^{\text {st }}$ to the $4^{\text {th }}$ cycle, which could be because of solid electrolyte interface (SEI) layer formation. The $1^{\text {st }}$ to $4^{\text {th }} \mathrm{CV}$ curves at a scan rate of $0.2 \mathrm{mV} \mathrm{s}^{-1}$ almost overlap, suggesting good cycling and stability after the first several cycles (Figure S5.5, Appendix). Galvanostatic charge/discharge curves also show the capacity decrease during the first to third cycles (Figure 5.5b). Three plateaus were observed in discharge curves that correspond to the cathodic peaks in Figure 5.5a.

Kinetics analysis was performed to further explore the surface and bulk contributions to the electrochemical performance of printed $\mathrm{V}_{2} \mathrm{O}_{5} / \mathrm{Ti}_{3} \mathrm{C}_{2} \mathrm{~T}_{\mathrm{x}}$ cathodes. Figure $5.5 \mathrm{c}$ shows the $\mathrm{CV}$ curves of printed $\mathrm{V}_{2} \mathrm{O}_{5} / \mathrm{Ti}_{3} \mathrm{C}_{2} \mathrm{~T}_{\mathrm{x}}$ cathodes at scan rates from 0.1 to $1 \mathrm{mV} \mathrm{s}^{-1}$. The surface capacitive effect can be determined by calculating the value of $b$ through the relation of $i=$ $a v^{b}$ where $i$ and $v$ are the current and scan rate, $a$ and $b$ are adjustable parameters. ${ }^{19}$ When the $b$ value is close to 0.5 , the electrochemical process is dominated by an ionic diffusion control mechanism. A $b$ value close to 1 indicates a surface capacitive mechanism. ${ }^{19}$ Figure $5.5 \mathrm{~d}$ 
plots the $\log i$ versus $\log v$ linear relationships at their peak potentials. The $b$ values of peaks $1,2,3,4,5,6$ are $0.65,0.70,0.69,0.57,0.66$ and 0.51 , respectively, suggesting synergistic contributions by both diffusion control and surface capacitive processes. Moreover, the current response $(i)$ at fixed potential $(\mathrm{V})$ can be separated into surface capacitive $\left(k_{1} v\right)$ and diffusion control mechanisms $\left(k_{2} v^{1 / 2}\right)$ using the equation $i(V)=k_{1} v+k_{2} v^{1 / 2} \cdot{ }^{19,20}$ Figure $5.5 \mathrm{e}$ shows the surface capacitive current (red region), compared with the total current in the $\mathrm{CV}$ profile at scan rate of $0.1 \mathrm{mV} \mathrm{s}^{-1}$. Around $49 \%$ of the total current comes from surface capacitive contributions, indicating that surface capacitive processes contribute almost half of the total current. It is noticeable that the calculated surface capacitive contribution is out of the range of the total $\mathrm{CV}$ curve in several parts, which could be explained by considering that the calculated surface capacitive contribution from CV curves is an ideal calculation. The dynamic resistance in a real electrochemical process could led to hysteresis in the current response. ${ }^{21}$ Furthermore, the surface capacitive contributions increase to $75 \%$ when the scan rate increases to $1 \mathrm{mV} \mathrm{s}^{-1}$, suggesting that the surface capacitive dominates the electrochemical process at high scan rates, which is beneficial for rate performance (Figure $5.5 f)$.

Moreover, the printed $\mathrm{V}_{2} \mathrm{O}_{5} / \mathrm{Ti}_{3} \mathrm{C}_{2} \mathrm{~T}_{\mathrm{x}}$ cathodes show good rate performance, as shown in Figure 5.6a. After an initial five cycle capacity decrease possibly because of SEI layer formation, stable capacities of 321, 245, 185, 124 and $112 \mathrm{mAh} \mathrm{g}^{-1}$ were achieved at 1, 2, 4, 8 and $10.5 \mathrm{C}$, respectively. More importantly, a high capacity of $112 \mathrm{mAh} \mathrm{g}^{-1}$ was achieved even at high $\mathrm{C}$ rate of $10.5 \mathrm{C}$ with high capacity retention of $65.7 \%$ compared to $321 \mathrm{mAh} \mathrm{g}^{-}$ ${ }^{1}$ at $1 \mathrm{C}$, indicating the high rate performance of the printed $\mathrm{V}_{2} \mathrm{O}_{5} / \mathrm{Ti}_{3} \mathrm{C}_{2} \mathrm{~T}_{\mathrm{x}}$ electrode. The corresponding individual galvanostatic charge/discharge curves are shown in Figure 5.6b. Three plateaus are visible at around 3.3, 3.2 and 2.3 V for discharge curves, corresponding to peaks 6, 5 and 4 in CV curves (Figure 5.5a). Cycling performance was conducted at 10.5 $\mathrm{C}$ as shown in Figure 5.5c-d. The half-cell showed good stable performance after around 680 cycles with a capacity retention of $91.7 \%$ and a coulombic efficiency of $96.5 \%$. As shown in Figure $5.5 \mathrm{e}$, the average capacity fading rate is about $0.01 \%$ per cycle which is lower than the other $\mathrm{V}_{2} \mathrm{O}_{5}$-based cathodes (Table S5.1). ${ }^{22-31}$ Moreover, printed $\mathrm{V}_{2} \mathrm{O}_{5} / \mathrm{Ti}_{3} \mathrm{C}_{2} \mathrm{~T}_{\mathrm{x}}$ cathodes exhibit a capacity as high as $112 \mathrm{mAh} \mathrm{g}^{-1}$ at current density as high as $3000 \mathrm{~mA} \mathrm{~g}^{-1}$. The excellent rate and cycling performances of the printed $\mathrm{V}_{2} \mathrm{O}_{5} / \mathrm{Ti}_{3} \mathrm{C}_{2} \mathrm{~T}_{\mathrm{x}}$ cathode are beneficial from following aspect: the large specific surface area of $2 \mathrm{D}_{2} \mathrm{O}_{5}$ nanosheets facilitates lithium ion diffusion during intercalation and deintercalation; the high surface capacitive contribution enables high capacity at high scan rate; the excellent electronic conductivity $\mathrm{Ti}_{3} \mathrm{C}_{2} \mathrm{~T}_{\mathrm{x}}$ nanosheets in the heterostructure electrodes enables electron transport; and the layerby-layer heterostructure of printed nanosheets cathode minimizes the volume change of cathode during charging and discharging processes. 

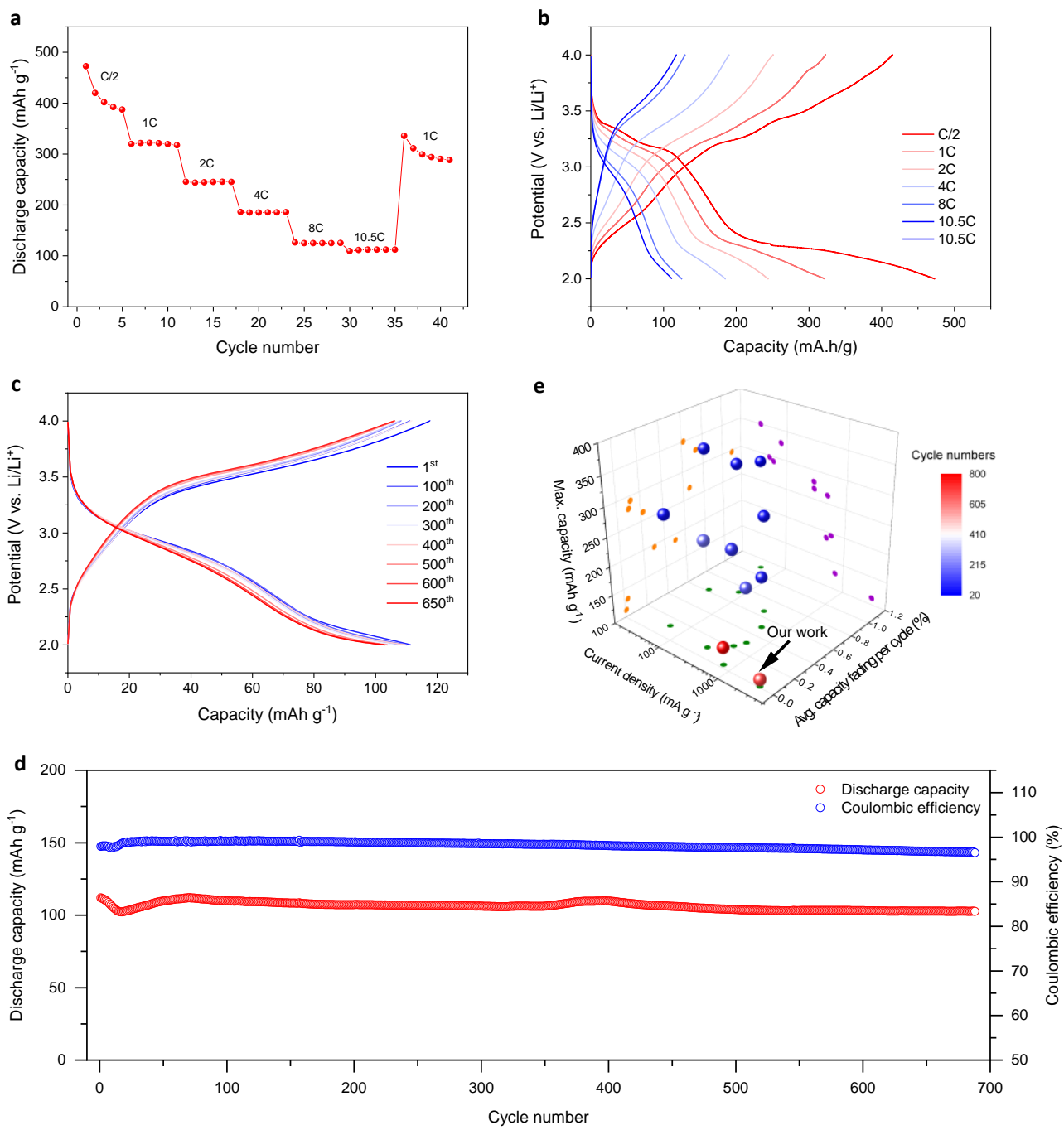

Figure 5.6 (a) Discharge capacity of printed $\mathrm{V}_{2} \mathrm{O}_{5} / \mathrm{Ti}_{3} \mathrm{C}_{2} \mathrm{~T}_{\mathrm{x}}$ cathodes at different discharge rates. (b) Galvanostatic charge/discharge curves of printed $\mathrm{V}_{2} \mathrm{O}_{5} / \mathrm{Ti}_{3} \mathrm{C}_{2} \mathrm{~T}_{\mathrm{x}}$ cathodes at different discharge rates. (c,d) Cycling performance of printed $\mathrm{V}_{2} \mathrm{O}_{5} / \mathrm{Ti}_{3} \mathrm{C}_{2} \mathrm{~T}_{\mathrm{x}}$ cathodes at 10.5C. (e) Comparison of the electrochemical performance of printed $\mathrm{V}_{2} \mathrm{O}_{5} / \mathrm{Ti}_{3} \mathrm{C}_{2} \mathrm{~T}_{\mathrm{x}}$ cathodes and $\mathrm{V}_{2} \mathrm{O}_{5}$-based composite cathodes illustrated by 3D scatter bubble plots of maximum capacity $\left(\mathrm{mAh} \mathrm{g}^{-1}\right)$, measured current density $\left(\mathrm{mA} \mathrm{g}^{-1}\right)$ and average capacity fading per cycle $(\%)$. The colors of the bubbles show the cycle numbers. 


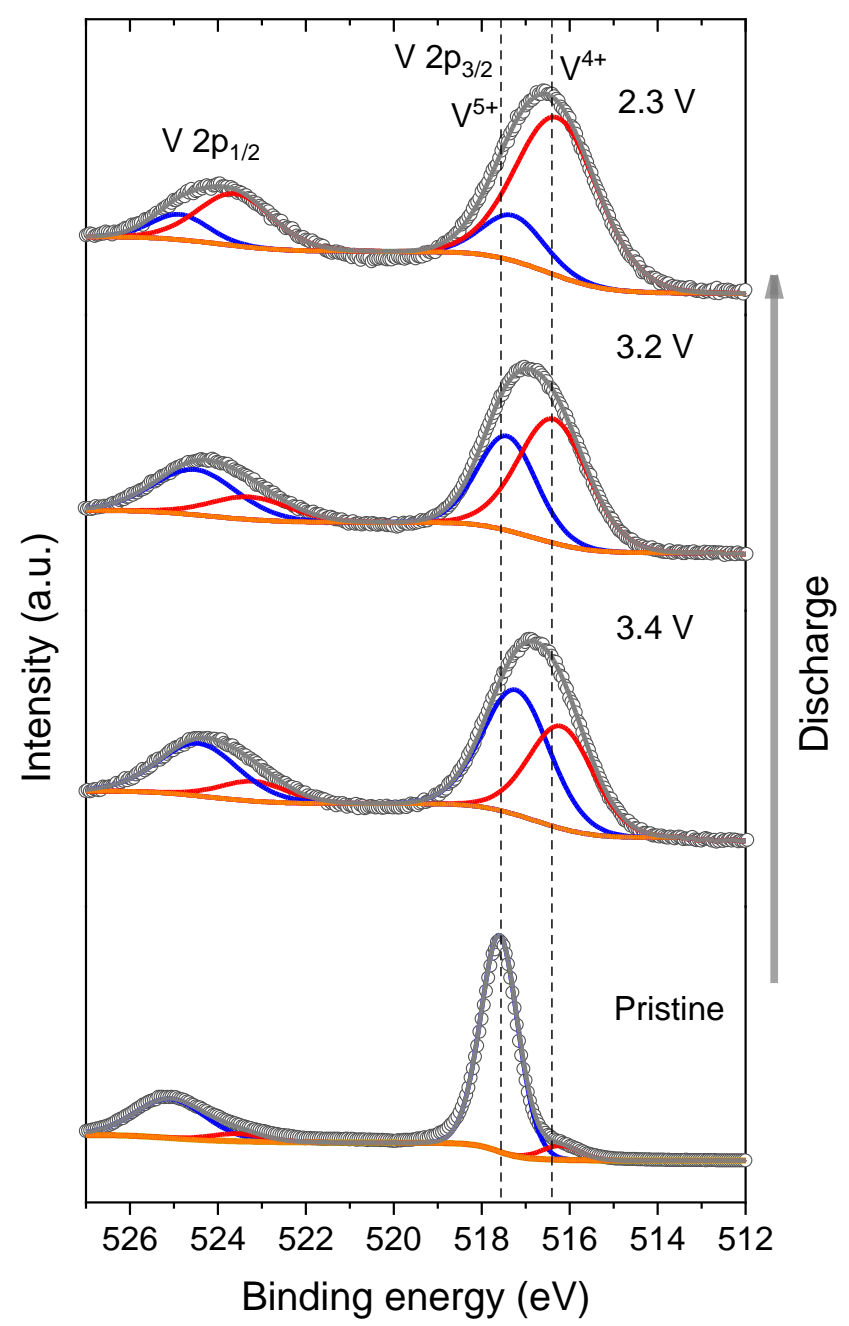

Figure 5.7 Ex-situ XPS spectra of V $2 p$ collected at different discharge plateaus in LIBs. The dashed lines indicate different oxidation states of vanadium.

Ex-situ XPS spectra were recorded to analyze how the oxidation state of vanadium changes in printed $\mathrm{V}_{2} \mathrm{O}_{5} / \mathrm{Ti}_{3} \mathrm{C}_{2} \mathrm{~T}_{\mathrm{x}}$ cathodes during the discharge process. As shown in Figure 5.7, the pristine $\mathrm{V} 2 p_{3 / 2}$ spectrum shape shows a strong $\mathrm{V}^{5+}$ peak and a small amount of $\mathrm{V}^{4+}$. When the LIB was discharged to $3.4 \mathrm{~V}$, the XPS spectrum of V $2 p$ could be deconvoluted into two spin-orbit doublets which are $\mathrm{V}^{4+}$ and $\mathrm{V}^{5+}$, indicating the oxidation state change of vanadium upon $\mathrm{Li}$ ion intercalation. It is noted that the $\mathrm{V}^{4+}$ peak has a comparable intensity as $\mathrm{V}^{5+}$ at $3.4 \mathrm{~V}$. The amount of $\mathrm{V}^{4+}$ increases further when discharged to $3.2 \mathrm{~V}$, suggesting further $\mathrm{Li}$ ion intercalation. When discharged to $2.3 \mathrm{~V}$, the amount of $\mathrm{V}^{4+}$ is much larger than that of 
$\mathrm{V}^{5+}$, indicating Li ion intercalations at $2.3 \mathrm{~V}$. Therefore, the ex-situ XPS spectra confirm the $\mathrm{Li}$ ion intercalation processes by analyzing the oxidation state of vanadium in printed $\mathrm{V}_{2} \mathrm{O}_{5} / \mathrm{Ti}_{3} \mathrm{C}_{2} \mathrm{~T}_{\mathrm{x}}$ cathodes at different discharge plateaus.

\subsection{Conclusions}

A water-based $2 \mathrm{D} \mathrm{V}_{2} \mathrm{O}_{5} / \mathrm{Ti}_{3} \mathrm{C}_{2} \mathrm{~T}_{\mathrm{x}}$ composite ink was inkjet printed to fabricate thin film cathodes for LIBs. The printed cathode was composed of a layer-by-layer structure, combining the advantageous characteristics of high theoretical capacity $\mathrm{V}_{2} \mathrm{O}_{5}$ and high electrical conductivity $\mathrm{Ti}_{3} \mathrm{C}_{2} \mathrm{~T}_{\mathrm{x}}$ nanosheets. Benefiting from the combination of the high capacity $\mathrm{V}_{2} \mathrm{O}_{5}$ nanosheets and the high electronic conductivity of $\mathrm{Ti}_{3} \mathrm{C}_{2} \mathrm{~T}_{\mathrm{x}}$ nanosheets, the printed heterostructure cathode exhibited high capacity and long cycling stability when operated as a LIB. Therefore, inkjet printed two-dimensional heterostructure-based electrodes with layer-by-layer structure open a new opportunity to high electrochemical performance batteries.

\subsection{Reference}

1. Pomerantseva, E., Bonaccorso, F., Feng, X., Cui, Y. \& Gogotsi, Y. Energy storage: The future enabled by nanomaterials. Science 366, eaan8285 (2019).

2. Peng, L., Zhu, Y., Chen, D., Ruoff, R. S. \& Yu, G. Two-dimensional materials for beyond-lithium-ion batteries. Adv. Energy Mater. 6, 1600025 (2016).

3. El-Kady, M. F., Shao, Y. \& Kaner, R. B. Graphene for batteries, supercapacitors and beyond. Nat. Rev. Mater. 1, 16033 (2016).

4. Chhowalla, M. et al. The chemistry of two-dimensional layered transition metal dichalcogenide nanosheets. Nat. Chem. 5, 263-275 (2013).

5. He, P., Yu, H., Li, D. \& Zhou, H. Layered lithium transition metal oxide cathodes towards high energy lithium-ion batteries. J. Mater. Chem. 22, 3680-3695 (2012).

6. Yue, Y. \& Liang, H. Micro- and nano-structured vanadium pentoxide $\left(\mathrm{V}_{2} \mathrm{O}_{5}\right)$ for electrodes of lithium-ion batteries. Adv. Energy Mater. 7, 1602545 (2017).

7. Kelly, A. G. et al. All-printed thin-film transistors from networks of liquidexfoliated nanosheets. Science 356, 69-73 (2017).

8. Pomerantseva, E. \& Gogotsi, Y. Two-dimensional heterostructures for energy storage. Nature Energy 2, 17089 (2017).

9. Anasori, B., Lukatskaya, M. R. \& Gogotsi, Y. 2D metal carbides and nitrides 
(MXenes) for energy storage. Nat. Rev. Mater. 2, 16098 (2017).

10. Zhang, Y. Z. et al. MXene printing and patterned coating for device applications. Adv Mater 32, 1908486 (2020).

11. Liang, S. et al. Template-free synthesis of ultra-large $\mathrm{V}_{2} \mathrm{O}_{5}$ nanosheets with exceptional small thickness for high-performance lithium-ion batteries. Nano Energy 13, 58-66 (2015).

12. Fontenot, C. J., Wiench, J. W., Pruski, M. \& Schrader, G. L. Vanadia gel synthesis via peroxovanadate precursors. 1. in situ laser Raman and 51V NMR characterization of the gelation process. J. Phys. Chem. B 104, 11622-11631 (2000).

13. Oka, Y., Yao, T., Yamamoto, N., Ueda, Y. \& Hayashi, A. Phase Transition and $\mathrm{V}^{4+}$ $\mathrm{V}^{4+}$ Pairing in $\mathrm{VO}_{2}(\mathrm{~B})$. J. Solid State Chem. 105, 271-278 (1993).

14. Enjalbert, R. \& Galy, J. A refinement of the structure of $\mathrm{V}_{2} \mathrm{O}_{5}$. Acta Crystallogr. C 42, 1467-1469 (1986).

15. Ureña-Begara, F., Crunteanu, A. \& Raskin, J.-P. Raman and XPS characterization of vanadium oxide thin films with temperature. Appl. Surf. Sci. 403, 717-727 (2017).

16. Silversmit, G., Depla, D., Poelman, H., Marin, G. B. \& De Gryse, R. Determination of the V 2p XPS binding energies for different vanadium oxidation states $\left(\mathrm{V}^{5+}\right.$ to $\mathrm{V}^{0+}$ ). J. Electron Spectros. Relat. Phenomena 135, 167-175 (2004).

17. Mendialdua, J., Casanova, R., Barbaux, Y. J. J. o. E. S. \& Phenomena, R. XPS studies of $\mathrm{V}_{2} \mathrm{O}_{5}, \mathrm{~V}_{6} \mathrm{O}_{13}, \mathrm{VO}_{2}$ and $\mathrm{V}_{2} \mathrm{O}_{3}$. J. Electron Spectros. Relat. Phenomena 71 , 249-261 (1995).

18. Lipatov, A. et al. Effect of Synthesis on Quality, Electronic properties and environmental stability of individual monolayer $\mathrm{Ti}_{3} \mathrm{C}_{2}$ MXene flakes. Adv. Electron. Mater. 2, 1600255 (2016).

19. Wang, J., Polleux, J., Lim, J. \& Dunn, B. Pseudocapacitive contributions to electrochemical energy storage in $\mathrm{TiO}_{2}$ (Anatase) nanoparticles. J. Phys. Chem. C 111, 14925-14931 (2007).

20. Liu, T. C. Behavior of molybdenum nitrides as materials for electrochemical capacitors. J. Electrochem. Soc. 145, 1882 (1998).

21. Tian, Z. et al. Printable magnesium ion quasi-solid-state asymmetric supercapacitors for flexible solar-charging integrated units. Nat. Commun. 10, 4913 (2019). 
22. Wang, Z.-1., Xu, D., Wang, L.-m. \& Zhang, X.-b. Facile and low-cost synthesis of large-area pure $\mathrm{V}_{2} \mathrm{O}_{5}$ nanosheets for high-capacity and high-rate lithium storage over a wide temperature range. ChemPlusChem 77, 124-128 (2012).

23. Li, Y. et al. Leaf-like $\mathrm{V}_{2} \mathrm{O}_{5}$ nanosheets fabricated by a facile green approach as high energy cathode material for lithium-ion batteries. Adv. Energy Mater. 3, 1171-1175 (2013).

24. Rui, X. et al. Ultrathin $\mathrm{V}_{2} \mathrm{O}_{5}$ nanosheet cathodes: realizing ultrafast reversible lithium storage. Nanoscale 5, 556-560 (2013).

25. Cheng, J. et al. Self-assembled $\mathrm{V}_{2} \mathrm{O}_{5}$ nanosheets/reduced graphene oxide hierarchical nanocomposite as a high-performance cathode material for lithium ion batteries. J. Mater. Chem. A 1, 10814-10820 (2013).

26. Wang, X. et al. Simple in situ synthesis of carbon-supported and nanosheetassembled vanadium oxide for ultra-high rate anode and cathode materials of lithium ion batteries. J. Mater. Chem. A 4, 13907-13915 (2016).

27. Zhu, D., Liu, H., Lv, L., Yao, Y. D. \& Yang, W. Z. Hollow microspheres of $\mathrm{V}_{2} \mathrm{O}_{5}$ and $\mathrm{Cu}$-doped $\mathrm{V}_{2} \mathrm{O}_{5}$ as cathode materials for lithium-ion batteries. Scr. Mater. 59, 642-645 (2008).

28. Cheah, Y. L., Aravindan, V. \& Madhavi, S. Improved elevated temperature performance of Al-intercalated $\mathrm{V}_{2} \mathrm{O}_{5}$ electrospun nanofibers for lithium-ion batteries. ACS Appl. Mater. Interfaces 4, 3270-3277 (2012).

29. Rui, X. et al. Facile preparation of hydrated vanadium pentoxide nanobelts based bulky paper as flexible binder-free cathodes for high-performance lithium ion batteries. RSC Advances 1, 117-122 (2011).

30. Zhang, Y. et al. Dodecahedron-Shaped Porous Vanadium Oxide and Carbon Composite for High-Rate Lithium Ion Batteries. ACS Appl. Mater. Interfaces 8 , 17303-17311 (2016).

31. Rahman, M. M. et al. Self-assembled $\mathrm{V}_{2} \mathrm{O}_{5}$ interconnected microspheres produced in a fish-water electrolyte medium as a high-performance lithium-ion-battery cathode. Nano Research 8, 3591-3603 (2015). 


\section{Appendices}

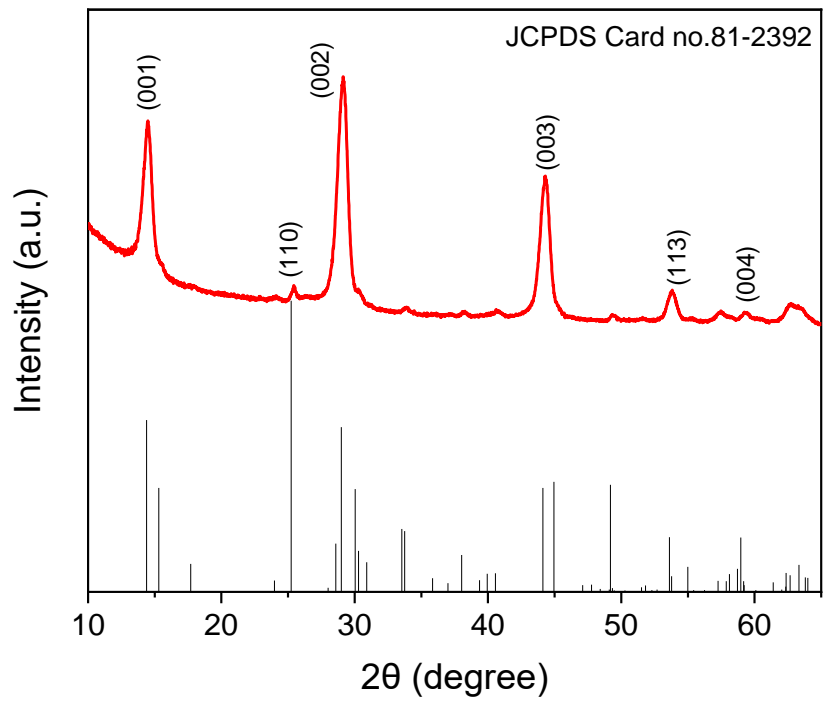

Figure S5.1 XRD spectra of $\mathrm{VO}_{2}$ (B) nanosheet film and reference diffractogram (JCPDS 81-2392).

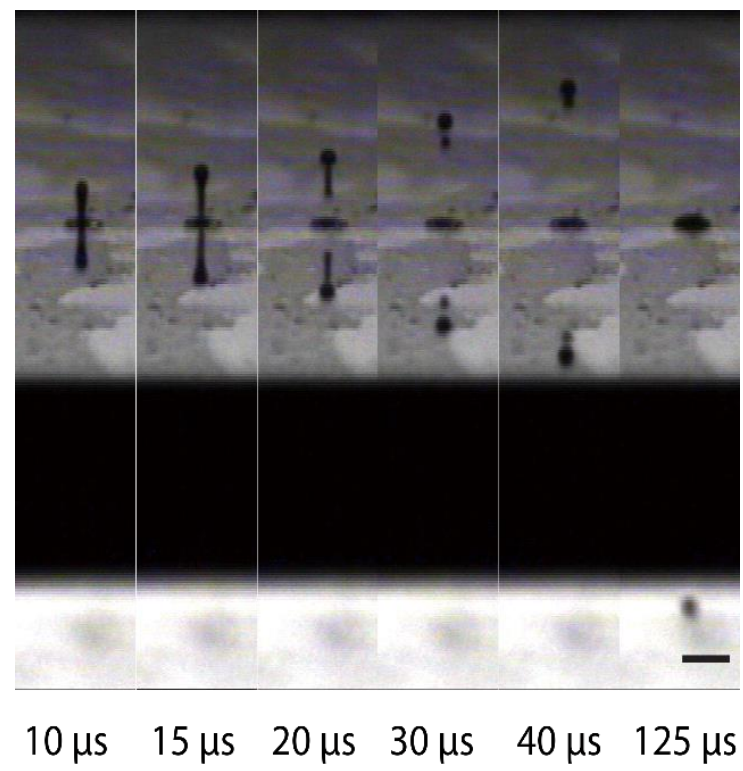

Figure S5.2 Optical image of $\mathrm{V}_{2} \mathrm{O}_{5} / \mathrm{Ti}_{3} \mathrm{C}_{2} \mathrm{~T}_{\mathrm{x}}$ composite ink droplet formation versus time as observed from the printer camera. The scale bar is $50 \mu \mathrm{m}$. 


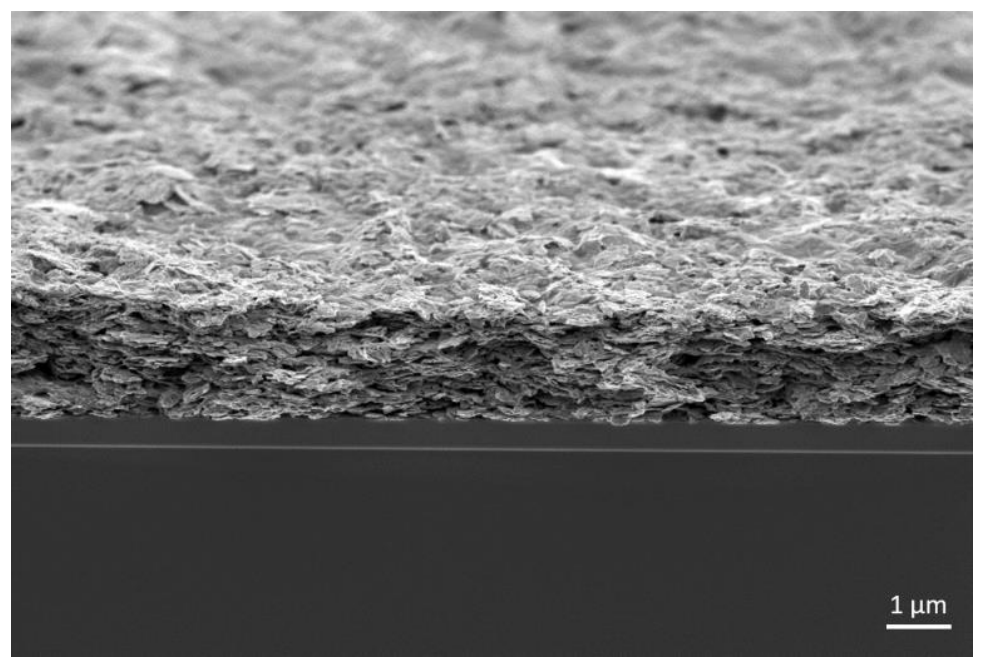

Figure S5.3 Cross-sectional SEM image of printed $\mathrm{V}_{2} \mathrm{O}_{5} / \mathrm{Ti}_{3} \mathrm{C}_{2} \mathrm{~T}_{\mathrm{x}}$ electrodes with thickness of around $1.8 \mu \mathrm{m}$.

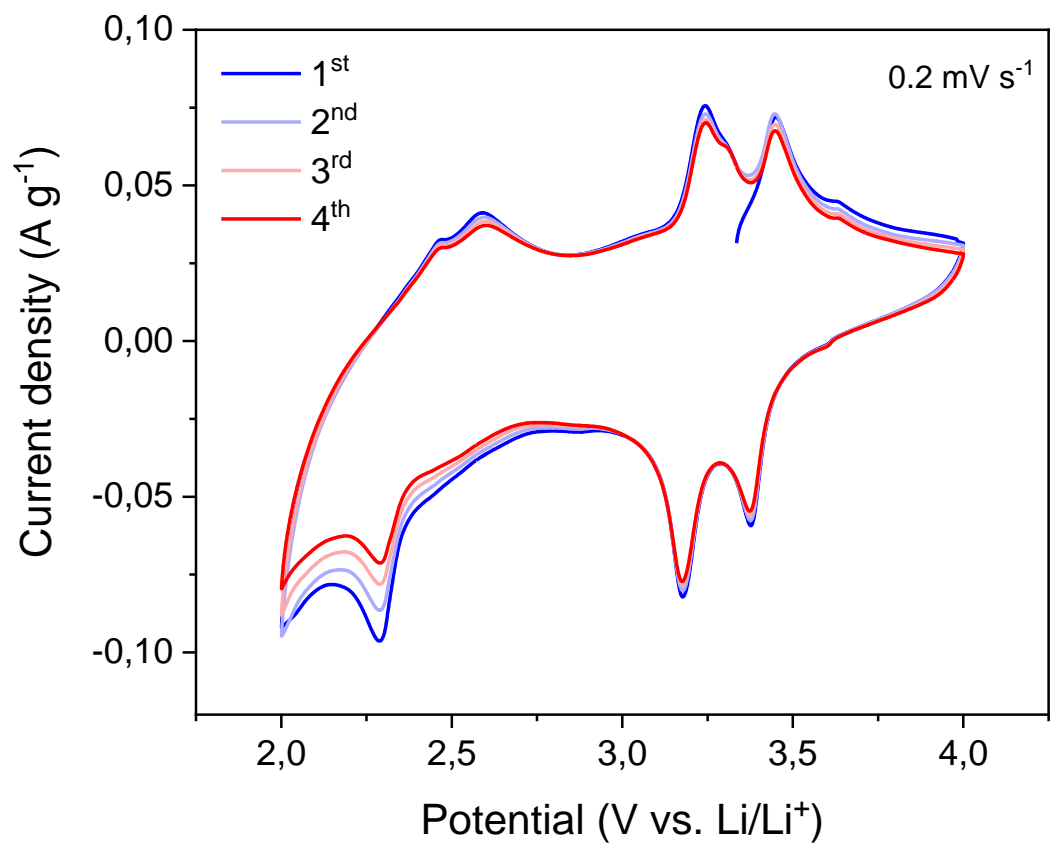

Figure S5.4 First to fourth cycle of voltammetric sweep of printed $\mathrm{V}_{2} \mathrm{O}_{5}$ nanosheet cathode at a scan rate of $0.2 \mathrm{mV}$ $\mathrm{s}^{-1}$ between 2 and $4 \mathrm{~V}$. 


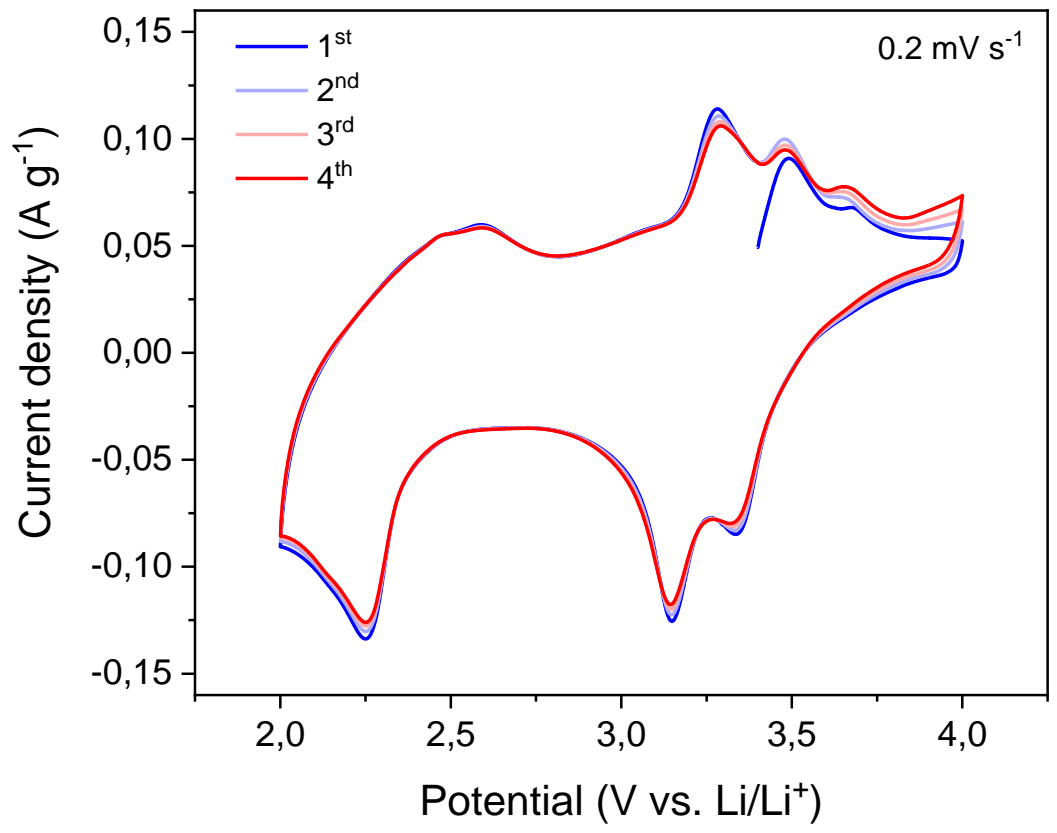

Figure S5.5 First to fourth cycle of voltammetric sweep of printed $\mathrm{V}_{2} \mathrm{O}_{5} / \mathrm{Ti}_{3} \mathrm{C}_{2} \mathrm{~T}_{\mathrm{x}}$ cathode at a scan rate of $0.2 \mathrm{mV} \mathrm{s}$ ${ }^{1}$ between 2 and $4 \mathrm{~V}$. 
Table S5.1 A comparison of the electrochemical performance of printed $\mathrm{V}_{2} \mathrm{O}_{5} / \mathrm{Ti}_{3} \mathrm{C}_{2} \mathrm{~T}_{\mathrm{x}}$ heterostructure cathode with other reported $\mathrm{V}_{2} \mathrm{O}_{5}$-based electrodes.

\begin{tabular}{|c|c|c|c|c|c|}
\hline Materials & $\begin{array}{c}\text { Max. } \\
\text { capacity } \\
\left(\mathrm{mAh} \mathrm{g}^{-1}\right)\end{array}$ & $\begin{array}{l}\text { Current } \\
\text { density } \\
\left(\mathrm{mA} \mathrm{g}^{-1}\right)\end{array}$ & $\begin{array}{c}\text { Cycle } \\
\text { number }\end{array}$ & $\begin{array}{c}\text { Average } \\
\text { capacity fading } \\
\text { per cycle }(\%)\end{array}$ & Reference \\
\hline $\begin{array}{c}\text { 2D large-area } \\
\mathrm{V}_{2} \mathrm{O}_{5} \\
\text { nanosheets }\end{array}$ & 268 & 300 & 50 & 0.66 & 22 \\
\hline $\begin{array}{l}\text { 2D leaf-like } \\
\qquad \mathrm{V}_{2} \mathrm{O}_{5} \\
\text { nanosheets }\end{array}$ & 264 & 500 & 100 & 0.22 & 23 \\
\hline $\begin{array}{l}\text { 2D ultrathin } \\
\qquad \mathrm{V}_{2} \mathrm{O}_{5} \\
\text { nanosheets }\end{array}$ & 292 & 59 & 50 & 0.12 & 24 \\
\hline $\mathrm{V}_{2} \mathrm{O}_{5}-\mathrm{rGO}$ & 196 & 600 & 160 & 0.3 & 25 \\
\hline $2 \mathrm{D} \mathrm{V}_{2} \mathrm{O}_{5} @ \mathrm{C}$ & 189 & 500 & 60 & 0.5 & 26 \\
\hline $\mathrm{V}_{2} \mathrm{O}_{5-} \mathrm{Cu}$ & 328 & 80 & 45 & 0.733 & 27 \\
\hline $\mathrm{V}_{2} \mathrm{O}_{5}-\mathrm{Al}$ & 350 & 35 & 50 & 0.62 & 28 \\
\hline $\begin{array}{c}1 \mathrm{D} \mathrm{V_{2 } \mathrm { O } _ { 5 }} \bullet \\
0.44 \mathrm{H}_{2} \mathrm{O} \\
\text { nanobelt }\end{array}$ & 297 & 50 & 20 & 1.1 & 29 \\
\hline $\mathrm{V}_{2} \mathrm{O}_{5-} \mathrm{C}$ & 130 & 735 & 800 & 0.03 & 30 \\
\hline $\begin{array}{c}3 \mathrm{D} \\
\text { interconnected } \\
\text { microspheres } \\
\text { composed of } \\
\text { ultra-long } \mathrm{V}_{2} \mathrm{O}_{5} \\
\text { nanobelts }\end{array}$ & 280 & 294 & 200 & 0.1 & 31 \\
\hline $\begin{array}{l}\mathrm{V}_{2} \mathrm{O}_{5} / \mathrm{Ti}_{3} \mathrm{C}_{2} \mathrm{~T}_{\mathrm{x}} \\
\text { heterostructure }\end{array}$ & 112 & 3000 & 688 & 0.01 & Our work \\
\hline
\end{tabular}


Chapter 5 


\section{Chapter 6}

\section{Challenges and Opportunities}

The research described in this thesis demonstrates that inkjet printing of two-dimensional materials as electroactive materials shows great promise for energy storage devices. However, there are challenges remaining to fabricate high performance printed energy storage devices and integrate them into portable electronics. Therefore, there are new opportunities for the scientific community to further explore printing two-dimensional materials for energy storage devices. 
In this thesis, a range of $2 \mathrm{D}$ nanosheets including doped and undoped $\mathrm{MnO}_{2}, \mathrm{MXene}$, graphene oxide and $\mathrm{V}_{2} \mathrm{O}_{5}$ have been synthesized and inkjet printed as active materials for energy storage devices including SCs and LIBs. As was shown in the previous chapters, inkjet printed SCs and LIBs show good electrochemical performance, and can find application in (flexible) devices. However, several issues are worth exploring further, and new opportunities are coming.

\subsection{Materials synthesis}

Exploring new low-cost exfoliation methods is crucial for mass production of 2D materials which is beneficial for inkjet printing. In my research, transition metal oxides nanosheets were used as active materials for SCs and LIBs because of their theoretical specific capacitance and capacity. However, enhancing the electronic conductivity is a big challenge for transition metal oxide nanosheets. Improving electronic conductivity by atomic doping shows great promise for high performance energy storage devices as demonstrated in this thesis. Although defect engineering of $\mathrm{MnO}_{2}$ nanosheets by substitutional doping of $3 d$ metal ions ( $\mathrm{Fe}, \mathrm{Co}$ and $\mathrm{Ni}$ ) has been demonstrated in this thesis, there is still an opportunity to further enhance $\mathrm{MnO}_{2}$ nanosheets' electronic conductivity and electrochemical performance like capacitance by doping with other potential ions. To enhance the $\mathrm{V}_{2} \mathrm{O}_{5}$ nanosheet conductivity, atomic doping is also a potential strategy, which is worth exploring further. First principles calculations are recommended to have deep understanding of the effect of doping on the band structure of $\mathrm{V}_{2} \mathrm{O}_{5}$ nanosheets. Synthesizing MXenes nanosheets with controllable surface terminations is crucial for electrochemical device performances. It is worth to investigate the electrochemical performance of MXene SCs with different surface terminations groups.

\subsection{Ink formulation}

The availability of materials for inkjet printing is still low because an inkjet printer requires inks that exhibit certain rheological properties, which implies strict requirements on ink viscosity, surface tension and the maximum size of 2D materials, etc. For instance, a low viscosity ink with small lateral size $2 \mathrm{D}$ materials is suitable for inkjet printing while a high viscosity ink with large lateral size 2D materials could block the nozzle during printing. Therefore, it remains challenging to prepare different 2D materials based printable ink with suitable rheological properties.

Moreover, highly stable inks without aggregation suited for long term storage are necessary for industrial production. It would also be interesting to investigate the relationship between the printed device performance and the lateral size of $2 \mathrm{D}$ materials.

\subsection{Printed energy storage devices}

One of the challenges for printed energy storage devices is printing resolution, which brings 
about critical issues like misalignment and poor quality of printed patterns. High resolution printing is essential to achieve good electrochemical performance of micro-supercapacitors. The minimum gap space between the interdigitated electrodes as demonstrated in this thesis is around $500 \mu \mathrm{m}$ which can be further decreased to shorten the electrolyte ion transport paths to improve rate performance. Digital inkjet printing technique enables us to set the gap space in software before printing. However, short connecting of electrodes will occur when the gap space is too small. One possible strategy to avoid shorts at high resolution printing is to control the drying process and facilitate fast solidification of printed patterns. Another possible strategy is to optimize the contact angle between ink and substrate. Therefore, it is both a challenge and an opportunity to fabricate devices with resolution as high as possible.

The quality of printed films is another key issue to achieve high performance energy storage devices. The common coffee-ring phenomenon should be avoided in printed devices by tuning the rheological properties of the inks and the energy of the substrate surface. As shown in chapter 2, surfactant and propylene glycol were used to optimize the surface tension and viscosity of the water-based ink, respectively. These additives may also work in other waterbased inks or even organic solvents. However, the additive concentration in different inks should be investigated carefully to obtain homogeneously printed films.

Moreover, the adhesion between printed patterns and substrates is also crucial for flexible energy storage devices. Surface pre-treatment of substrates or inks modification are two strategies to achieve good adhesion. Post-treatment is also crucial for flexible substrates since most of them cannot withstand high temperature.

A highly horizontal device orientation is preferred for micro-supercapacitors to short electrolyte ion diffusion between in-plane electrodes resulting in high power density device. Therefore, printed film with a controllable structure is worth for further study. Printing pinhole free films is a challenge for both SCs and LIBs fabrication. Furthermore, integrate inkjet printing process into a roll-to-roll printing is a challenge for the industrial production of printed energy storage devices and integrated function devices.

Two-dimensional heterostructures for energy storage devices attract more and more attention recently. The inkjet printing technique shows great advantages like simple and low-cost for the fabrication of large-scale 2D heterostructures compared with conventional dry transfer methods. The heterostructure interface is crucial to achieve high performance devices. An intimate and stable interface contact between the MXene/GO/MXene heterostructure has been demonstrated in this thesis, while the contact at the cross-section between the interdigitated MXene electrode and the GO electrolyte was poor in the GO/MXene heterostructure. It is worth to address these poor contact issues to achieve better performance for GO/MXene heterostructure micro-supercapacitors. Moreover, realizing good contacts between different 2D materials other than MXene and GO nanosheets in heterostructures is 
worth to further explore.

\subsection{Printed energy storage devices performance}

Achieving high energy/power density, high safety and long cycling life are the goals for energy storage devices of SCs and batteries. However, the cycling stability of printed $\mathrm{MnO}_{2}$ nanosheets SCs in this thesis did not reach expectation. One possible reason is that $\mathrm{MnO}_{2}$ nanosheets store charges by surface redox reactions while long cycling charge/discharge processes will damage its $2 \mathrm{D}$ structure, resulting in decreasement of capacitance and poor cycling life. The loosen contact between 2D nanosheets in printed nanosheets electrodes is another possible reason. The long cycling charging/discharging measurements could increase the interspace between $2 \mathrm{D}$ nanosheets resulting in poor contact and poor electrochemical performance. It would be interesting to address the poor cycling stability that does not only exist in printed $\mathrm{MnO}_{2}$-based SCs, but also in other transition metal oxide nanosheet-based SCs. The fast self-discharge phenomenon limits the SCs in devices application. It is worth investigating how to inhibit the self-discharge process in the SCs.

The mechanical stability under bending is also crucial for flexible energy storage devices. A crack formed in printed nanosheet-based SCs after repeated bending experiments as demonstrated in this thesis, results in electrochemical performance deterioration. It is therefore a big challenge to keep excellent contact between neighboring 2D nanosheets and the mechanical stability of printed 2D nanosheet films after extended bending tests.

Poor cycling stability is also a major issue for LIBs. Stacking different 2D nanosheets into heterostructure electrodes shows promise to address the above issue. The layer-by-layer heterostructure of printed nanosheet electrodes could minimize the volume change of the electrode during charging and discharging, resulting in long cycling life. Therefore, it is worth investigating different 2D material heterostructure electrodes for LIBs. 


\section{Summary}

2D materials have been attracting more and more attention for energy storage devices including supercapacitors and batteries because of their unique physical and chemical properties induced by dimensional reduction. The aim of research presented in this thesis was to develop a deep understanding of devices' electrochemical performance with 2D materials and address some of the challenges currently faced by the printed $2 \mathrm{D}$ electronics field.

$2 \mathrm{D} \delta-\mathrm{MnO}_{2}$ nanosheets which shows high theoretical specific capacitance were firstly studied as electroactive materials for micro-supercapacitors in this thesis. A printable water-based $\delta$ $\mathrm{MnO}_{2}$ nanosheet ink was developed to demonstrate the possibility of inkjet printing 2D nanosheets (Chapter 2). Due to the excellent dispersibility in water, the $\delta-\mathrm{MnO}_{2}$ nanosheets could be formulated directly in water-based inks. Triton X-100 and propylene glycol were added into $\delta-\mathrm{MnO}_{2}$ nanosheets suspension to optimize the surface tension and viscosity, respectively. By optimizing the concentration of Triton X-100, coffee-ring and droplet shrinking effects were avoided by balancing Marangoni flow and outward flow. The thickness dependence of the specific capacitance of $\delta-\mathrm{MnO}_{2}$ films was investigated by varying printed $\delta-\mathrm{MnO}_{2}$ films thickness. As a proof-of-concept application, all-solid-state symmetrical micro-supercapacitors based on $\delta-\mathrm{MnO}_{2}$ nanosheets with interdigitated electrode configurations were fabricated. The fabricated micro-supercapacitor showed excellent mechanical flexibility and good cycling stability with a capacitance retention of $88 \%$ after 3600 charge-discharge cycles. The micro-supercapacitor attained the highest volumetric capacitance of $2.4 \mathrm{~F} \mathrm{~cm}^{-3}$, and an energy density of $1.8 \cdot 10^{-4} \mathrm{Wh} \mathrm{cm}^{-3}$ at a power density of $0.018 \mathrm{~W} \mathrm{~cm}^{-3}$, which is comparable with other devices and shows great potential as energy storage units for low-cost flexible and wearable electronics applications.

However, the poor electronic conductivity of $\delta-\mathrm{MnO}_{2}$ nanosheets limits the devices electrochemical performance like power density. To improve the electronic conductivity of $\delta-\mathrm{MnO}_{2}$ nanosheets, defect engineering of $\mathrm{MnO}_{2}$ nanosheets by atomic-level substitutional doping of $3 d$ metal ions ( $\mathrm{Fe}, \mathrm{Co}$ and $\mathrm{Ni}$ ) was investigated (Chapter 3). The doped $\mathrm{MnO}_{2}$ nanosheets were printed as electroactive materials to show the effect of doping on materials electrochemical performance. By introducing 5\% Fe doping, occupied and unoccupied impurity states emerge in the fundamental band gap so that the chemical potential is increased, which effectively decreases the band gap and increases the mobile charge carrier density. Only empty impurity states form in the band gap for $\mathrm{Ni}$ and Co doped systems and these do not contribute much to the carrier density. Substitutional doping introduces new electronic states near the Femi level, thereby enhancing the electronic conductivity and contributing to 
the formation of redox-active $3 d$ surface states. All-solid-state symmetrical microsupercapacitors based on Fe-doped $\mathrm{MnO}_{2}$ nanosheets were fabricated. The microsupercapacitors exhibited high volumetric energy density of up to $1.13 \times 10^{-3} \mathrm{Wh} \mathrm{cm}^{-3}$ at a power density of $0.11 \mathrm{~W} \mathrm{~cm}^{-3}$ which increased by a factor of more than 6 comparing with undoped $\mathrm{MnO}_{2}$ nanosheets micro-supercapacitors. Moreover, the micro-supercapacitors show an ultra-small leakage current.

A gel electrolyte was used in chapter 2 and 3 to fabricate solid-state micro-supercapacitors. However, I argue that 2D materials can also act as a solid-state electrolyte and separator for full solid-state supercapacitors. 2D nanosheets show various interesting properties like metallic conductivity, semiconductivity and insulating behavior. Heterostructures constructed from different 2D nanosheet building blocks show promise for energy storage applications, since materials with different properties can be combined. Such 2D heterostructures were fabricated by inkjet printing MXene and graphene oxide (GO) nanosheets into a stacking structure (Chapter 4). $\mathrm{Ti}_{3} \mathrm{C}_{2} \mathrm{~T}_{\mathrm{x}}$ (MXene) nanosheets that show excellent electronic conductivity and excellent dispersibility in water were printed as electrode materials. GO nanosheets were printed as solid-state electrolyte owning to their high ionic conductivity and poor electronic conductivity. The mobile protons needed for charging/discharging these devices arose from the hydrolysis of functional oxygen-bearing groups on the solid GO electrolyte. Free water molecules present between GO sheets may facilitate proton transport via the Grotthuss mechanism or by diffusion of hydronium ions within the interlayer spaces. By controlling the printing parameters, an intimate and stable contact between the MXene electrodes and the GO electrolyte was obtained in a MXene/GO/MXene heterostructure, which facilitated proton transfer between GO and MXene nanosheets. The contact at the cross-section between the interdigitated MXene electrode and the GO electrolyte was poor in the GO/MXene heterostructure, resulting in poor devices performance without liquid electrolyte. On the other hand, the printed MXene/GO/MXene heterostructure without liquid electrolyte showed a higher areal energy density of $0.49 \mu \mathrm{Wh} \mathrm{cm} \mathrm{cm}^{-2}$ at an areal power density of $12.55 \mu \mathrm{W} \mathrm{cm} \mathrm{cm}^{-2}$, comparing with existing printed supercapacitors. Moreover, the areal specific capacitance could be increased further by adding a liquid electrolyte.

Enhancing power density and cycling life are crucial for a good battery. Due to the distinct electronic properties, shortened ion diffusion paths and more active site capability, an inkjet printed 2D materials heterostructure as cathode for a lithium-ion battery was studied in detail (Chapter 5). 2D V $\mathrm{O}_{5}$ nanosheet shows great promise as cathode material for LIBs because of their high theoretical capacity of $294.8 \mathrm{mAh} \mathrm{g}^{-1}$ with two lithium ion intercalations per unit cell in the potential window from 2 to $4 \mathrm{~V}$. High electronic conductivity $\mathrm{Ti}_{3} \mathrm{C}_{2} \mathrm{~T}_{\mathrm{x}} \mathrm{MXene}$ nanosheets were used as charge transport carriers in the printed $\mathrm{V}_{2} \mathrm{O}_{5} / \mathrm{MXene}$ heterostructure. The printed cathode exhibited high capacity of $321 \mathrm{mAh} \mathrm{g}^{-1}$ at $1 \mathrm{C}$, high-rate capacities of $112 \mathrm{mAh} \mathrm{g}^{-1}$ at $10.5 \mathrm{C}$ and good cycling stabilities after 680 cycles with $91.8 \%$ capacity 
retention, indicating high electrochemical performance. Therefore, an inkjet printed 2D heterostructure electrode with layer-by-layer structure open new opportunities to high electrochemical performance batteries.

The research described in this thesis has shown that printed 2D materials as electroactive materials show great promise for energy storage devices. However, challenges remain for further exploration in this exciting area. Luckily, the good news is that there are lots of opportunities worth exploring in future (Chapter 6). The inkjet printing technique is a special catalyst that enables us to transform ideas to real devices. 


\section{Samenvatting}

2D materialen trekken steeds meer aandacht voor energieopslagapparaten, waaronder supercondensatoren en batterijen, vanwege hun unieke fysische en chemische eigenschappen, dat wordt veroorzaakt door dimensionaliteitsreductie. Het doel van het onderzoek dat in dit proefschrift wordt gepresenteerd, was om een diep begrip te krijgen van de elektrochemische prestaties van apparaten met 2D materialen en om enkele van de uitdagingen aan te gaan die het geprinte 2D elektronicaveld momenteel onder ogen ziet.

2D $\delta-\mathrm{MnO}_{2}$ nanosheets die een hoge theoretische specifieke capaciteit laten zien, werden in dit proefschrift allereerst bestudeerd als elektroactieve materialen voor microsupercapacitors. Een printbare $\delta-\mathrm{MnO}_{2}$ nanosheet-inkt op waterbasis was ontwikkeld om de mogelijkheid van inkjet printen van 2D nanosheets aan te tonen (Hoofdstuk 2). Vanwege de uitstekende dispergeerbaarheid in water, konden de $\delta-\mathrm{MnO}_{2}$ nanosheets rechtstreeks voor inkten op waterbasis gebruikt worden. Triton X-100 en propyleenglycol werden toegevoegd aan $\delta-\mathrm{MnO}_{2}$ nanosheet-suspensies om respectievelijk de oppervlaktespanning en viscositeit te optimaliseren. Door de concentratie van Triton X-100 te optimaliseren, werden koffie-ring en druppel-krimpeffecten voorkomen door Marangoni-stromen en uitgaande stromen in evenwicht te houden. De dikte-afhankelijkheid van de specifieke capaciteit van $\delta-\mathrm{MnO}_{2}$ films werd onderzocht door de dikte van geprinte $\delta-\mathrm{MnO}_{2}$ films te variëren. Als proof-of-concept applicatie, werden volledig-vaste-stof symmetrische microsupercondensatoren vervaardigd op basis van $\delta-\mathrm{MnO}_{2}$ nanosheets met onderling verweven elektrodeconfiguraties. De gefabriceerde microsupercondensator vertoonde uitstekende mechanische flexibiliteit en goede cyclus-stabiliteit met een capaciteitsbehoud van $88 \%$ na 3600 laad-ontlaadcycli. De microsupercondensator bereikte de hoogste volumetrische capaciteit van $2.4 \mathrm{~F} \mathrm{~cm}^{-3}$ en een energiedichtheid van $1.8 \cdot 10^{-4} \mathrm{Wh} \mathrm{cm}^{-3}$ bij een vermogensdichtheid van $0.018 \mathrm{~W} \mathrm{~cm}^{-3}$, wat vergelijkbaar is met andere apparaten en laat grote potentie zien als energieopslag voor goedkope, flexibele en draagbare elektronicatoepassingen.

De zwakke elektronische geleidbaarheid van $\delta-\mathrm{MnO}_{2}$ nanosheets beperkt de elektrochemische prestaties van apparaten, zoals vermogensdichtheid. Om de elektronische geleidbaarheid van $\delta-\mathrm{MnO}_{2}$ nanosheets te verbeteren, werd defect-engineering van $\mathrm{MnO}_{2}$ nanosheets door substitutie-dotering op atomair niveau van $3 d$-metaalionen (Fe, Co en $\mathrm{Ni}$ ) onderzocht (Hoofdstuk 3). De gedoteerde $\mathrm{MnO}_{2}$ nanosheets werden geprint als elektroactieve materialen om het effect van doping op de elektrochemische prestaties van het materiaal te laten zien. Door 5\% Fe-doping te introduceren, ontstaan bezette en onbezette onzuiverheidstoestanden in de fundamentele band gap, zodat het chemische potentieel wordt 
vergroot, wat de band gap effectief verkleint en de dichtheid van de mobiele ladingsdrager verhoogt. Er vormen zich alleen lege onzuiverheidstoestanden in de band gap voor systemen gedoteerd met $\mathrm{Ni}$ en $\mathrm{Co}$ en deze dragen niet veel bij aan de dragerdichtheid. Substitutionele doping introduceert nieuwe elektronische toestanden nabij het Femi-niveau, waardoor de elektronische geleidbaarheid wordt verbeterd en wordt bijgedragen aan de vorming van redox-actieve $3 d$ oppervlaktetoestanden. Er werden volledig-vaste-stof symmetrische microsupercondensatoren vervaardigd op basis van Fe-gedoteerde $\mathrm{MnO}_{2}$ nanosheets. De microsupercondensatoren vertoonden een hoge volumetrische energiedichtheid tot $1.13 \cdot 10^{-3}$ Wh cm$~^{-3}$ bij een vermogensdichtheid van $0.11 \mathrm{~W} \mathrm{~cm}^{-3}$, die met een factor van meer dan 6 toenam in vergelijking met ongedoteerde $\mathrm{MnO}_{2}$ nanosheets microsupercondensatoren. Bovendien vertonen de microsupercondensatoren een ultrakleine lekstroom.

In Hoofdstuk 2 en $\mathbf{3}$ werd een gel-elektrolyt gebruikt om vaste-stof microsupercondensatoren te fabriceren. Ik betoog echter dat 2D materialen ook kunnen werken als een vaste-stof elektrolyt en scheider voor volledig-vaste-stof supercondensatoren. 2D nanosheets tonen verschillende interessante eigenschappen zoals metallische geleiding, halfgeleiding en isolatiegedrag. Heterostructuren opgebouwd uit bouwstenen van verschillende $2 \mathrm{D}$ nanosheets zijn veelbelovend voor energieopslagtoepassingen, omdat materialen met verschillende eigenschappen kunnen worden gecombineerd. Dergelijke 2D heterostructuren zijn vervaardigd door MXene en grafeenoxide (GO) nanosheets te inkjet printen tot een stapelstructuur (Hoofdstuk 4). $\mathrm{Ti}_{3} \mathrm{C}_{2} \mathrm{~T}_{\mathrm{x}}$ (MXene) nanosheets die uitstekende elektronische geleidbaarheid en uitstekende dispergeerbaarheid in water vertonen, werden geprint als elektrodematerialen. GO nanosheets werden geprint als vaste-stof elektrolyt die hoge ionische geleidbaarheid en slechte elektronische geleidbaarheid bezit. De mobiele protonen die nodig zijn voor het laden / ontladen van deze apparaten zijn ontstaan door de hydrolyse van functionele zuurstofhoudende groepen op de vaste GO elektrolyt. Vrije watermoleculen die tussen GO platen aanwezig zijn, kunnen het transport van protonen vergemakkelijken via het Grotthuss mechanisme of door diffusie van hydroniumionen in de tussenlaagruimten. Door de print-parameters te regelen, werd een diep en stabiel contact tussen de MXene elektroden en de GO elektrolyt verkregen in een MXene/GO/Mxene heterostructuur, wat de protonoverdracht tussen GO en MXene nanosheets vergemakkelijkte. Het contact bij de dwarsdoorsnede tussen de onderling verweven MXene elektrode en de GO elektrolyt was pover in de GO/Mxene heterostructuur, dat resulteerde in slechte prestaties van de apparaten zonder vloeibare elektrolyt. Aan de andere kant vertoonde de geprinte heterogene heterostructuur van MXene/GO/MXene zonder vloeibare elektrolyt een hogere oppervlakte energiedichtheid van $0.49 \mu \mathrm{Wh} \mathrm{cm}^{-2}$ met een oppervlakte vermogensdichtheid van $12.55 \mu \mathrm{W} \mathrm{cm}{ }^{-2}$, vergelijkbaar met bestaande geprinte supercondensatoren. Bovendien zou de oppervlaktespecifieke capaciteit verder kunnen worden vergroot door een vloeibare elektrolyt toe te voegen.

Het verbeteren van de vermogensdichtheid en de levensduur is cruciaal voor een goede 
batterij. Vanwege de verschillende elektronische eigenschappen, verkorte ionendiffusiepaden en actievere locatiemogelijkheden, werd een heterostructuur met inkjet geprinte 2D materialen (ook) als kathode voor een lithium-ionbatterij in detail bestudeerd (Hoofdstuk 5). 2D $\mathrm{V}_{2} \mathrm{O}_{5}$ nanosheet is veelbelovend als kathodemateriaal voor LIB's vanwege hun hoge theoretische capaciteit van $294.8 \mathrm{mAh} \mathrm{g}^{-1}$ met twee lithiumionintercalaties per eenheidscel in het potentie venster van 2 tot $4 \mathrm{~V}$. Hoge elektronische geleidbaarheid $\mathrm{Ti}_{3} \mathrm{C}_{2} \mathrm{~T}_{\mathrm{x}}$ MXene nanosheets werden gebruikt als ladingsdragers in de geprinte $\mathrm{V}_{2} \mathrm{O}_{5} / \mathrm{MXene}$ heterostructuur. De geprinte kathode vertoonde een hoge capaciteit van $321 \mathrm{mAh} \mathrm{g}^{-1}$ bij $1 \mathrm{C}$, hoge capaciteit van $112 \mathrm{mAh} \mathrm{g}^{-1}$ bij $10.5 \mathrm{C}$ en goede cyclusstabiliteit na 680 cycli met een behoud van capaciteit van $91.8 \%$, dat wijst op hoge elektrochemische prestaties. Daarom opent een met inkjet geprinte 2D heterostructuurelektrode met laag-voor-laagstructuur nieuwe mogelijkheden voor batterijen met hoge elektrochemische prestaties.

Het onderzoek beschreven in dit proefschrift heeft aangetoond dat gedrukte 2D-materialen als elektroactieve materialen veelbelovend zijn voor energieopslagapparaten. Er blijven echter uitdagingen bestaan voor verdere verkenning in dit opwindende gebied. Hoewel het goede nieuws is dat er in de toekomst veel mogelijkheden zijn die het ontdekken waard zijn (Hoofdstuk 6). De inkjet print techniek is een speciale katalysator die ons in staat stelt ideeën om te zetten in echte apparaten. 


\section{Scientific Outputs}

\section{List of publications}

1. Wang, Y.; Lubbers, T.; Xia, R.; Mehrali, M.; Huijben, M.; ten Elshof, J. E., Printed twodimensional $\mathrm{V}_{2} \mathrm{O}_{5} / \mathrm{MXene}$ heterostructure cathode for lithium-ion batteries. (Submitted)

2. Wang, Y.; Mehrali, M.; Zhang, Y.-Z.; Timmerman, M. A.; Boukamp, B. A.; Xu, P.-Y.; ten Elshof, J. E., Tunable capacitance in all-inkjet-printed nanosheet heterostructures. 2020, arXiv preprint arXiv:2004.03350.

3. Wang, Y.; Zhang, Y.-Z.; Gao, Y.-Q.; Sheng, G.; ten Elshof, J. E., Defect engineering of $\mathrm{MnO}_{2}$ nanosheets by substitutional doping for printable solid-state microsupercapacitors. Nano Energy 2020, 68, 104306.

4. Wang, Y.; Zhang, Y.-Z.; Dubbink, D.; ten Elshof, J. E., Inkjet printing of $\delta-\mathrm{MnO}_{2}$ nanosheets for flexible solid-state micro-supercapacitor. Nano Energy 2018, 49, 481488.

5. Zhang, Y.-Z.;† Wang, Y. (co-first author) $; \dagger$ Jiang, Q. $; \dagger$ K. EI-Demellawi, J.; Kim, H.; Alshareef, H. N., MXene printing and patterned coating for device applications. Advanced Materials 2020, 32, 1908486.

6. Zhang, Y.-Z.;† Wang, Y. (co-first author); $\dagger$ Cheng, T.;† Yao, L.-Q.; Li, X.-C.; Lai, W.Y.; Huang, W., Printed supercapacitors: materials, printing and applications. Chemical Society Reviews 2019, 48, 3229-3264.

7. ten Elshof, J. E.; Wang, Y., * Advances in ink-jet printing of $\mathrm{MnO}_{2}$-nanosheet based pseudocapacitors. Small Methods 2019, 3 (8), 1800318. (*corresponding author)

8. Zhang, Y.-Z.; Wang, Y.; Cheng, T.; Lai, W.-Y.; Pang, H.; Huang, W., Flexible supercapacitors based on paper substrates: a new paradigm for low-cost energy storage. Chemical Society Reviews 2015, 44 (15), 5181-5199.

9. Jenkins, G.; Wang, Y.; Xie, Y. -L.; Wu, Q.; Huang, W.; Wang, L.; Yang, X., Printed electronics integrated with paper-based microfluidics: new methodologies for nextgeneration health care. Microfluidics and Nanofluidics 2015, 19 (2), 251-261.

10. Zhang, Y.-Z.; Wang, Y.; Xie, Y.-L.; Cheng, T.; Lai, W.-Y.; Pang, H.; Huang, W., Porous hollow $\mathrm{Co}_{3} \mathrm{O}_{4}$ with rhombic dodecahedral structures for high-performance supercapacitors. Nanoscale 2014, 6 (23), 14354-14359.

11. Xia, R.; Sun, C.; Wang, Y.; Cunha, D.; Peng, H.; Zhao, K.; Huijben. M.; ten Elshof, J. E., Enhanced lithiation dynamics in nanostructured $\mathrm{Nb}_{18} \mathrm{~W}_{16} \mathrm{O}_{93}$ anodes. Journal of Power Sources 2020 (Accepted)

12. Li, F.-C.; Ruan, C.-H.; Wang, Y.; Lei, L.-X., Ionic liquid-assisted in situ growth of a cobalt oxide composite and its application in supercapacitors and electrochemical biosensors. New Journal of Chemistry 2018, 42, 18659-18666.

13. Zhang, Y.-Z.; Cheng, T.; Wang, Y.; Lai, W.-Y.; Pang, H.; Huang, W., A simple approach 
to boost capacitance: flexible supercapacitors based on manganese oxides@MOFs via chemically induced in situ self-transformation. Advanced Materials 2016, 28 (26), 52425248 .

14. Timmerman, M. A.; Xia, R.; Le, P. T. P.; Wang, Y.; ten Elshof, J. E., Metal oxide nanosheets as 2D building blocks for the design of novel materials. Chemistry - A European Journal 2020, 26, 9084-9098.

\section{Conference presentations and posters}

1. Wang, Y.; Zhang, Y.-Z.; Gao, Y.-Q.; Sheng, G.; ten Elshof, J. E., Defect engineering of $\mathrm{MnO}_{2}$ nanosheets by substitutional doping for printable solid-state microsupercapacitors. 2019 E-MRS Spring Meeting, Symposium C, May 2019, Nice, France (Poster).

2. Wang, Y.; ten Elshof, J. E., 2D oxide nanosheets for energy storage devices. Technical University of Denmark, June 2019, Denmark (Oral).

3. Wang, Y.; ten Elshof, J. E., Inkjet printing of $\delta-\mathrm{MnO}_{2}$ nanosheets for flexible solid-state micro-supercapacitor. MESA+ Day, September 2018, Enschede, The Netherlands (Oral).

4. Wang, Y.; ten Elshof, J. E., Inkjet printing $\mathrm{MnO}_{2}$ nanosheets for flexible microsupercapacitors. 2018 E-MRS Spring Meeting, June 2018, Strasbourg, France (Oral).

5. Wang, Y.; Zhang, Y.-Z.; Lai, W.-Y.; Pang, H.; Huang, W.; ten Elshof, J. E., Printed electronics integrated with flexible supercapacitors: new methodologies for low-cost energy storage. 2017 E-MRS Spring Meeting, May 2017, Strasbourg, France (Poster).

\section{Peer review}

Reviewer for Advanced Functional Materials, Small. 


\section{Acknowledgement}

The $\mathrm{PhD}$ study journey approaches to the final station at this moment, while I am feeling that I just started this wonderful journey several weeks ago. Of course, it is not true. But it is time to say thank you to everyone who have helped and supported me during this journey.

Firstly, I would like to express my sincere gratitude and appreciation to my supervisor André ten Elshof, for giving me the opportunity to pursue my $\mathrm{PhD}$ in IMS group, and continuous encouragement and support of my PhD study. André, many thanks for your guidance, patience and knowledge in aspect of ideas discussion, data analyzing, paper and thesis writing. Thank you for the freedom you gave me to develop me own ideas during this wonderful PhD study. I enjoy discussing new ideas with you and always receiving your useful feedbacks and support. I learned a lot from you on how to do science. I would also like to appreciate Mark Huijben for giving me advices on battery research and discussion we had during battery group meeting.

I appreciate the collaboration with Dr. Yi-Zhou Zhang, Dr. Mohammad Mehrali and Dr. Yuqiang Gao during my PhD study. Yi-Zhou, my academic bro, we knew each other eight years ago when I just started my master study. I would like to express my appreciation to you for the suggestions you gave and collaboration we have. We had several high-impact publications from our collaboration. I look forward more collaboration in the future and wish you great success in establishing your own research group in Nanjing. Mohammad, our collaboration started from the discussion we had in the lab. I appreciate you for giving me so interesting materials which made last two chapters possible. I hope we can have more collaboration in the future. Yuqiang, many thanks for your effort to make the third chapter more convincing. I enjoy having collaboration with you because of your expertise in theoretical calculation. Hope we can have more collaboration in the future and wish you great success in your career.

I would like to appreciate my graduation committee members, Prof. Marnix Wagemaker, Prof. Bart J. Kooi, Prof. Mark Huijben, Prof. Rob Lammertink and Prof. Remko Akkerman for taking time to read my thesis and participate in the defence.

I would like to thank Marion, Jose for all type of paperwork and documents. Many thanks to Karin, Dominic, Laura and Harry for their help on the chemical ordering and equipment.

I want to express my special thanks for my paranymphs, Rui and Melvin. Rui, I enjoy the discussions with you on batteries. And thanks for your help with batteries measurement especially in situ XRD although we didn't get nice data. Wish you all the best with your PhD study. Melvin, thanks for your help to check my thesis summary in Dutch. Good luck on your PhD research. 
Huiyu, thanks for your help when I just started my $\mathrm{PhD}$ study in IMS group. I still remember that you invited me to have my first dinner in the Netherlands with your family members. Hope we can have collaboration in future. Binbin, I enjoy discussing with you on different topics during lunch time and the trips we had together with Rui to different countries. Wish you great success in your academia career.

I am very happy to be an IMSer and have opportunities to join two wonderful study trips. Many thanks go to all the past and present IMSer for their help. Thanks to all my officemates, Huiyu, Petra, Daniel, Thanh, Ron and Marijn, for the wonderful discussions we had in our offices. I would like to thank Bernard for his useful impendence course and help on analyzing my data. Thank Guus, Mark and Gertjan for their help and suggestions during my PhD study. I would like to thank Jun for his help on XPS measurements and talks we had during lunch time. Jie, thank you for the discussion we had on battery in the lab and wish you success in your PhD life. Special thanks go to my student Mohammad Torkamanzadeh and Ties Lubbers for their contributions to this thesis. There are still many people I would like to thank for their help: Evert, Monica, Rebecca, Ufuk, SiZhao, Minh, Deekpak, Yorick, Jaap, Sina, Kit, Moritz, Phu, David, Shu.....

Special thanks go to Mark Smithers (Nanolab) who made a lot of beautiful SEM images of $2 \mathrm{D}$ materials.

I want to thank all the friends I met in Enschede. Pengyu and Jin, we had our first trip in the Netherlands during our first Christmas holiday. Pengyu, many thank you for helping me to fix my bike and wish you great success in your career in Shanghai. Jin, wish you great success in your academic career in Hunan. And the other friends: Shanqiu Liu, Qirong Yao, Xiaozhen He, Ruosha Zeng, Jianfeng Huang, Zhaoliang, Zhengchao Guo, Zhiliang Qiao, Shiya Wu, Yan Liu, Juan Wang, Qian Li, Maria.....

At the end, I would like to thank my family for endless and unconditional supports and encouragement. I am grateful to my father (王永好) and mother (王永聪) who have provided me emotional support in my life. I would also like to thank parents-in-law for their supports. My beloved wife Tingting, you are meaning too much to me. It is because of you who made this wonderful journey funnier and more meaningful. The most grateful thanks to you. I am ready for our next adventure! 